\title{
(ब)
}

AUTARQUIA ASSOCIADA À UNIVERSIDADE DE SÃO PAULO

\section{RADIOATIVIDADE NATURAL, ELEMENTOS MAIORES E TRAÇOS DETERMINADOS EM PRODUTOS NACIONAIS DERIVADOS DA \\ Nicotiana tabacum $L$.}

Aline Sebastiane Gonçales Ramos de Oliveira

Dissertação apresentada como parte dos requisitos para obtenção do Grau de Mestre em Ciências na Área de Tecnologia Nuclear - Aplicações

Orientadora:

Profa. Dra. Sandra Regina Damatto 
INSTITUTO DE PESQUISAS ENERGÉTICAS E NUCLEARES

Autarquia associada à Universidade de São Paulo

\section{RADIOATIVIDADE NATURAL, ELEMENTOS MAIORES E TRAÇOS DETERMINADOS EM PRODUTOS DERIVADOS DA Nicotiana tabacum $L$.}

Aline Sebastiane Gonçales Ramos de Oliveira

Dissertação apresentada como parte dos
requisitos para obtenção do Grau de
Mestre em Ciências na Área
de Tecnologia Nuclear - Aplicações
Orientadora:
Profa. Dra. Sandra Regina Damatto
\#

Versão Corrigida

Versão Original disponível no IPEN

São Paulo

2017 
Dedico este trabalho primeiramente à DEUS, pois sem ele nada em minha vida seria possível e todos que acreditaram e me apoiaram durante essa jornada. 


\section{AGRADECIMENTOS}

A Deus por ter me sustentado todo esse tempo.

Ao Instituto de Pesquisas Energéticas e Nucleares e a Comissão Nacional de energia Nuclear pela oportunidade e condição para realizar este trabalho.

Ao Conselho Nacional de Desenvolvimento Científico e Tecnológico (CNPq) pelo apoio financeiro.

A minha orientadora, Dra. Sandra Regina Damatto, por todo conhecimento transmitido, dedicação, compreensão e paciência durante o desenvolvimento deste trabalho.

A Dra. Vera Lucia Salvador por todo ensinamento e colaboração no desenvolvimento desta pesquisa.

A minha família por todo apoio. Ao meu marido Rodrigo Fabiano meu companheiro e amigo. Ao Isaac Fabiano por toda a felicidade que ele me proporciona a cada manhã. À minha mãe Elizabel Ramos e a minha avó Eurides Barbosa por todo o empenho e dedicação a mim e a minha família durante essa caminhada.

A Dra. Bárbara Paci Mazzilli pela oportunidade de se realizar esse trabalho no Laboratorio de Radiometria Ambiental.

Aos amigos e companheiros de laboratório Arthur Athayde, André Rodrigo, Joseilton Marques, Levi Francisco, Amanda Prilip, Amanda Shiagasi, Denise Sanny, Laissa Adriana, Pedro Gonçalves e Thiago Santos.

Aos colegas do Laboratório de Radiometria Ambiental: Paulo Renê, Marcelo Máduar, Marcelo Bessa, Lucio Leonardo, Luiz Flavio, Marcos Medrado, Dra. Brigitte Pecequilo, Marcia de Campos e Cátia Saueia por todo apoio e incentivo.

A todos aqueles que acreditaram em mim.......

Muito Obrigado. 
Entrega o teu caminho ao Senhor; confia nele, e o mais ele fará.

Salmo 37:5

Mire na lua. Mesmo que você erre cairá entre as estrelas.

Les Brown 


\section{RESUMO}

de OLIVEIRA, ALINE S. G. R. Radioatividade natural, elementos maiores e traços determinados em produtos nacionais derivados da Nicotiana tabacum L. 2017. 174 p. Dissertação (Mestrado em Tecnologia Nuclear) - Instituto de Pesquisas Energéticas e Nucleares - IPEN- CNEN/SP, São Paulo.

O consumo de tabaco é uma das principais causas de doenças e morte prematuras no mundo; é a segunda droga mais consumida entre os adolescentes brasileiros, sendo considerado uma importante porta de entrada para o uso de drogas ilícitas. O Brasil é o segundo produtor mundial de tabaco e desde de 1993 destaca-se como o maior exportador. Os radionuclídeos naturais das séries do ${ }^{232} \mathrm{Th}$ e ${ }^{238} \mathrm{U}$ são encontrados no tabaco em baixas concentrações absorvidos diretamente do solo ou por deposição foliar. No ato de fumar ocorre a transferência desses radionuclídeos através da queima do tabaco para os tecidos e órgãos humanos podendo gerar lesões cancerígenas, sendo o mais relevante o câncer de pulmão. Existem poucos dados sobre a caracterização radiológica e elementar dos derivados do tabaco brasileiros, o que torna relevante o presente estudo que teve como objetivos a determinação da radioatividade natural e da concentração de elementos maiores e traços em derivados de tabaco produzidos e comercializados no Brasil. As técnicas analíticas empregadas foram alfa e beta total após separação radioquímica para os radionuclídeos ${ }^{226} \mathrm{Ra},{ }^{228} \mathrm{Ra}$ e ${ }^{210} \mathrm{~Pb}$ com determinação em detector proporcional de fluxo gasoso e baixa radiação de fundo, espectrometria alfa após separação radioquímica para o radionuclídeo ${ }^{210} \mathrm{Po}$, análise por ativação com nêutrons instrumental (INAA) e fluorescência por dispersão de raios $X$ (EDXRF) para determinação de ${ }^{238} \mathrm{U},{ }^{232} \mathrm{Th}$, elementos maiores e traços. Foram analisados produtos derivados da Nicotina tabacum L. de diferentes marcas adquiridas em tabacarias: cigarro não aromatizados, cigarro aromatizado, charuto, rapé, cigarro de palha e fumo de corda. Pela técnica de INAA foi possível determinar a concentração de 19 elementos e pela técnica de EDXRF de 31 elementos o que possibilitou uma ampla caracterização multielementar e as técnicas analíticas empregadas se mostraram complementares. Os elementos que apresentaram 
maiores valores de concentração foram o Ca e o $\mathrm{K}$ entre todas as amostras amostragem e entre todos os radionuclídeos naturais determinados o ${ }^{228} \mathrm{Ra}$ apresentou maiores valores de concentração de atividade. A partir da concentração de atividade determinada foram calculadas a dose anual estimada e a dose anual efetiva para os radionuclídeos ${ }^{210} \mathrm{~Pb}$ e ${ }^{210} \mathrm{Po}$, levando-se em consideração um consumo anual de $3,65 \mathrm{~kg}$ de tabaco por ano. A dose anual efetiva variou de 69,5 $\mu \mathrm{Sv}$ ano $^{-1}$ à $121 \mu \mathrm{Sv}$ ano $^{-1}$. Os produtos que apresentaram maiores valores de concentração e consequentemente maiores valores de dose anual efetiva, para a maioria dos radionuclídeos analisados, foram os cigarros de palha e fumos de corda.

Palavras-chave: Nicotiana tabacum L., INAA, EDXRF, radioquímica, dose efetiva anual, radioatividade natural 


\section{ABSTRACT}

de OLIVEIRA, ALINE S. G. R. Natural radioactivity, major and trace elements determined in Brazilian products derived from Nicotiana tabacum L. 2017. 174 p. Dissertação (Mestrado em Tecnologia Nuclear) - Instituto de Pesquisas energéticas e Nucleares - IPEN- CNEN/SP, São Paulo.

Tobacco use is one of the leading causes of premature illness and death in the world; is the second most consumed drug among Brazilian adolescents, being considered an important gateway to the use of illicit drugs. Brazil is the second largest tobacco producer in the world and since 1993 it has been the largest exporter. The natural radionuclides from the ${ }^{232} \mathrm{Th}$ and ${ }^{238} \mathrm{U}$ series are found in tobacco at low concentrations absorbed directly from the soil or by foliar deposition. In the act of smoking occurs the transference of these radionuclides through the burning of the tobacco to the human tissues and organs and they can generate carcinogenic lesions, being the lung cancer the most relevant. There are few data on the elemental and radiological characterization of Brazilian tobacco products, which makes relevant the present study that had as objectives the determination of the natural radioactivity and the concentration of major and trace elements in tobacco products produced and marketed in Brazil. The analytical techniques employed were gross alpha and beta, after radiochemical separation for the radionuclides ${ }^{226} \mathrm{Ra},{ }^{228} \mathrm{Ra}$ and ${ }^{210} \mathrm{~Pb}$ with determination in a gaseous flow proportional detector of low background radiation, alpha spectrometry after radiochemical separation for the radionuclide ${ }^{210} \mathrm{Po}$, instrumental neutron activation analysis (INAA) and X-ray scattering fluorescence (EDXRF) for determination of ${ }^{238} \mathrm{U},{ }^{232} \mathrm{Th}$, major and trace elements. Nicotine tabacum L. products from different brands acquired in cigar stores were analyzed: non-flavored cigarettes, flavored cigarettes, cigar, snuff, straw cigarettes and rope smoke. Using the INAA technique, it was possible to determine the concentration of 19 elements and with the EDXRF technique 31 elements, which enabled a wide multielementar characterization; the analytical techniques employed were complementary. The elements that presented the highest concentration values were $\mathrm{Ca}$ and $\mathrm{K}$ among all the samples and among 
all the natural radionuclides determined the ${ }^{228} \mathrm{R}$ a presented higher values of activity concentration. The estimated annual dose and annual effective dose for the ${ }^{210} \mathrm{~Pb}$ and ${ }^{210} \mathrm{Po}$ radionuclides were calculated from the determined activity concentration, taking into account an annual consumption of $3.65 \mathrm{~kg}$ of tobacco per year. The effective annual dose ranged from $69.5 \mu \mathrm{Sv} \mathrm{y}^{-1}$ to $121 \mu \mathrm{Sv} \mathrm{y}^{-1}$. The products with the highest concentration values and hence the highest annual effective dose for the majority of the radionuclides analyzed were straw cigarettes and rope smoke.

Key words: Nicotiana tabacum L., INAA, EDXRF, radiochemistry, annual effective dose, natural radioactivity 


\section{SUMÁRIO}

Página

1 INTRODUÇÃO

1.1 Tabagismo: questão de saúde pública.............................................24

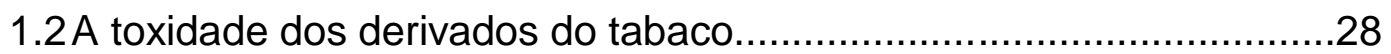

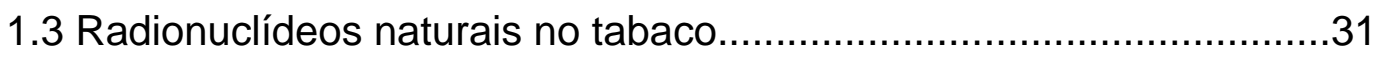

1 OBJETIVOS

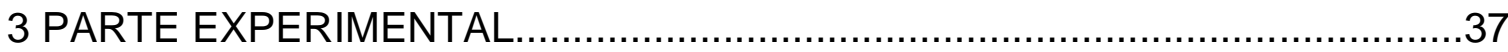

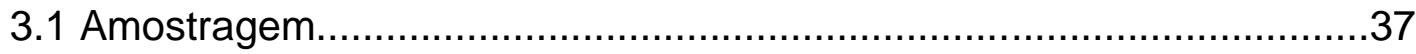

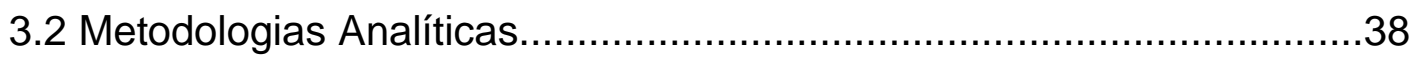

3.2.1 Análise por Ativação com Nêutrons Instrumental (INAA) ..........39

3.2.2 Espectrometria de Fluorescência de Raios-X (EDXRF).............43

3.2.3 Metodologia de determinação ${ }^{226} \mathrm{Ra},{ }^{228} \mathrm{Ra}$ e ${ }^{210} \mathrm{~Pb}$..................45

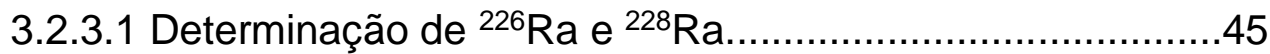

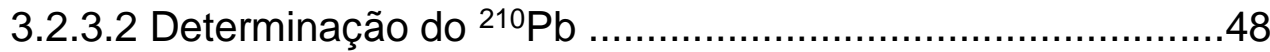

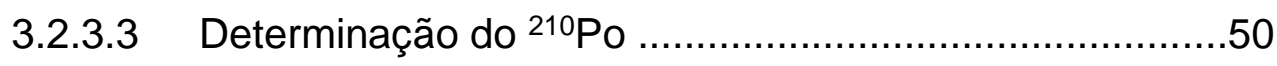

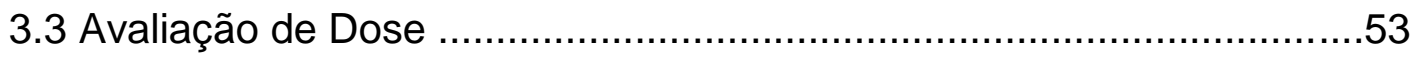

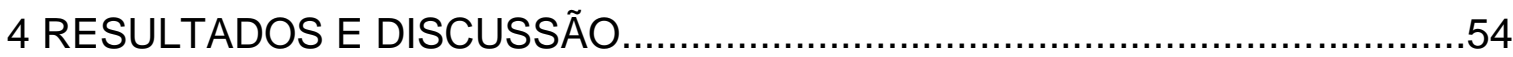

4.1 Análise por Ativação com Nêutrons Instrumental - INAA........................54

4.1.1 Precisão e exatidão da INAA...................................................54

4.1.2 Determinação do limite inferior de detecção (LID).....................59

4.2 Espectrometria de Fluorescência de Raios-X (EDXRF)........................61 
4.2.2 Determinação do limite de determinação do método (LDM).....65

4.3 Determinação da radiação de fundo .68

4.4 Determinação da eficiência de contagem para as medidas de ${ }^{226} \mathrm{Ra}$, ${ }^{228} \mathrm{Ra}$ e ${ }^{210} \mathrm{~Pb}$.

4.4.1 Determinação da eficiência de contagem alfa total para a medida de ${ }^{226} \mathrm{Ra}$ .68

4.4.2 Determinação da eficiência de contagem beta de ${ }^{226} \mathrm{Ra}$ para a medida de ${ }^{228} \mathrm{Ra}$..... 69

4.4.3 Determinação da eficiência de contagem beta de ${ }^{228} \mathrm{Ra}$..........70

4.4.4 Determinação da eficiência de contagem beta de ${ }^{210} \mathrm{~Pb}$............70

4.4.5 Determinação da eficiência do espectrômetro alfa. .71

4.4.6 Determinação da curva de calibração do espectrômetro alfa.

4.5 Determinação do limite inferior de detecção para as metodologias de dos radionuclídeos ${ }^{226} \mathrm{Ra},{ }^{228} \mathrm{Ra},{ }^{210} \mathrm{~Pb}$ e ${ }^{210} \mathrm{Po}$.

4.6 Verificação das metodologias. .73

4.7 Resultados das metodologias para os derivados da Nicotiana tabacum L .73

4.7.1 Análise de Ativação com Nêutrons Instrumental - INAA .74

4.7.1.1 Elementos maiores $\mathrm{Ca}, \mathrm{K}, \mathrm{Fe}$, e $\mathrm{Na}$ .75

4.7.1.2 Elementos Traço As, Ba, Br, Co, Cr, Cs, Hf, Rb, Sb, Se, Ta, $\mathrm{Th}, \mathrm{U}$ e $\mathrm{Zn}$. .79

4.7.1.3 Elementos Terras Raras Ce, Eu, La, Lu, Nd, Sc, Sm, Tb e $\mathrm{Yb}$.

4.7.2 Espectrometria de Fluorescência de Raios-X (EDXRF). .93

4.7.2.1 Elementos maiores $\mathrm{Al}, \mathrm{Ca}, \mathrm{Cl}, \mathrm{Fe}, \mathrm{K}, \mathrm{I}, \mathrm{Mg}, \mathrm{Mn}, \mathrm{P}$ e S.......93

4.7.2.2 Elementos traço As, Br, Cd, Co, Cr, Cs, Cu, Hg, Ni, Pb, Rb, $\mathrm{Sb}, \mathrm{Se}, \mathrm{Sr}, \mathrm{Th}, \mathrm{U}, \mathrm{V}$, e Zn. 101

4.7.2.3 Elementos Terras Raras La, Nd e Sm 
4.7.3 Radionuclídeos Naturais ${ }^{226} \mathrm{Ra},{ }^{210} \mathrm{~Pb},{ }^{210} \mathrm{Po},{ }^{238} \mathrm{U},{ }^{228} \mathrm{Ra} \mathrm{e}$ $232 \mathrm{Th}$

4.7.4 Avaliação da dose....................................................126

4.7.4.1 Dose estimada................................................126

4.7.4.2 Dose anual efetiva................................................128

4.8 Caracterização química inorgânica e radiológica dos derivados da

Nicotiana tabacum L. estudados..........................................................128

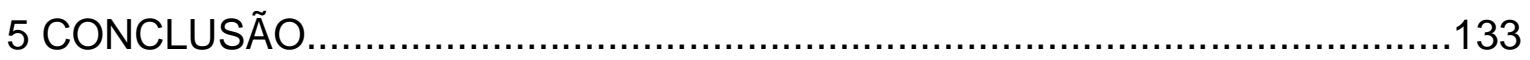

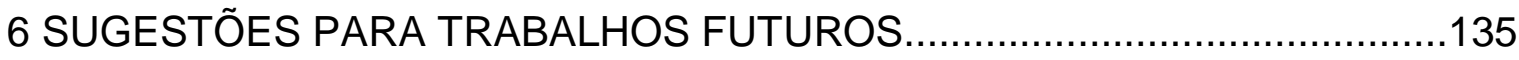

REFERÊNCIAS BIBLIOGRÁFICAS ................................................. 136

\section{APENDICE}

APÊNDICE A.1 Concentração dos elementos determinados em $\mathrm{mg} \mathrm{kg}^{-1}$ nos cigarros não aromatizados determinados pela técnica INAA;

APÊNDICE A.2 Concentração dos elementos determinados em $\mathrm{mg} \mathrm{kg}^{-1}$ nos cigarros aromatizados determinados pela técnica INAA;

APÊNDICE A.3 Concentração dos elementos determinados em $\mathrm{mg} \mathrm{kg}^{-1}$ nos cigarros de palha determinados pela técnica INAA;

APÊNDICE A.4 Concentração dos elementos determinados em $\mathrm{mg} \mathrm{kg}^{-1}$ nos charutos determinados pela técnica INAA;

149

APÊNDICE A.5 Concentração dos elementos determinados em $\mathrm{mg} \mathrm{kg}^{-1}$ nos fumos de corda e rapé determinados pela técnica INAA; 150 
APÊNDICE B.1 Concentração dos elementos determinados em $\mathrm{mg} \mathrm{kg}^{-1}$ nos cigarros não aromatizados determinados pela técnica EDXRF;

APÊNDICE B.2 Concentração dos elementos determinados em $\mathrm{mg} \mathrm{kg}^{-1}$ nos cigarros aromatizados determinados pela técnica EDXRF;

157

APÊNDICE B.3 Concentração dos elementos determinados em $\mathrm{mg} \mathrm{kg-}^{-1}$ nos cigarros de palha determinados pela técnica EDXRF;

APÊNDICE B.4 Concentração dos elementos determinados em $\mathrm{mg} \mathrm{kg}^{-1}$ nos charutos determinados pela técnica EDXRF;

APÊNDICE B.5 Concentração dos elementos determinados em $\mathrm{mg} \mathrm{kg}^{-1}$ nos fumos de corda e rapés determinados pela técnica EDXRF; 164

APÊNDICE C.1 Concentração dos radionuclídeos estudados em $\mathrm{mBq} \mathrm{g}^{-1}$ nos cigarros aromatizados;

APÊNDICE C.2 Concentração dos radionuclídeos estudados em $\mathrm{mBq} \mathrm{g}^{-1}$ nos cigarros não aromatizados;

APÊNDICE C.3 Concentração dos radionuclídeos estudados em $\mathrm{mBq} \mathrm{g}^{-1}$ nos cigarros de palha; 172

APÊNDICE C.4 Concentração dos radionuclídeos estudados em $\mathrm{mBq} \mathrm{g}^{-1}$ nos charutos; 172

APÊNDICE C.5 Concentração dos radionuclídeos estudados em $\mathrm{mBq} \mathrm{g}^{-1}$ nos fumos de corda e rapés. 173 


\section{LISTA DE TABELAS}

Página

TABELA 3.1 - Elementos determinados por análise por ativação neutrônica, concentrações dos elementos nos materiais de referência $\left(\mathrm{mg} \mathrm{kg}^{-1}\right)$, radioisótopos formados na ativação, energia dos raios gama utilizados na espectrometria gama e meias-vidas.

TABELA 3.2 - Concentrações nos materiais de referência ( $\mathrm{mg} \mathrm{kg}^{-1}$, *\%), usados na calibração e validação dos resultados pela análise por EDXRF.... 44

TABELA 4.1 - Concentrações médias calculadas e concentrações certificadas, em $\mathrm{mg} \mathrm{kg}^{-1}$, para o material de referência Soil 1 utilizando Montanna II e Tomato Leaves como padrões

TABELA 4.2 - Concentrações médias calculadas e concentrações certificadas, em $\mathrm{mg} \mathrm{kg}^{-1}$, para o material de referência Montanna II utilizando Soil 1 e Tomato Leaves como padrões. 56

TABELA 4.3 - Concentrações médias calculadas e concentrações certificadas, em $\mathrm{mg} \mathrm{kg}^{-1}$, para o material de referência Tomato Leaves utilizando Soil 1 e Montanna II como padrões.

TABELA 4.4 - Limites inferiores de determinação para os elementos estudados por INAA, em mg kg-1, para os materiais de referência Soil 1, Montanna II e Tomato Leaves. 60

TABELA 4.5 - Concentrações médias calculadas e concentrações certificadas, em $\mathrm{mg} \mathrm{kg}^{-1}(* \%)$, para o material de referência Peach Leaves. 62

TABELA 4.6 - Concentrações médias calculadas e concentrações certificadas, em $\mathrm{mg} \mathrm{kg}^{-1}\left({ }^{*} \%\right)$, para o material de referência Sargasso.

TABELA 4.7 - Concentrações médias calculadas e concentrações certificadas, em $\mathrm{mg} \mathrm{kg}^{-1}\left({ }^{*} \%\right)$, para o material de referência Tomato Leaves.

TABELA 4.8 - Limites de determinação do método para os elementos estudados por EDXRF, em mg kg-1 (* em \%), para os materiais de referencia Peach Leaves, Sargasso e Tomato Leaves.

TABELA 4.9 - Resultados obtidos para o LID dos radionuclídeos ${ }^{226} \mathrm{Ra},{ }^{228} \mathrm{Ra},{ }^{210} \mathrm{~Pb}$ $\mathrm{e}^{210} \mathrm{Po}$ em $\mathrm{mBq} \mathrm{g}{ }^{-1}$ (DPR) e erros relativo (ER)......

TABELA 4.10 - Valores de Atividade de concentração calculada e certificada dos radionuclídeos ${ }^{226} \mathrm{Ra},{ }^{228} \mathrm{Ra},{ }^{210} \mathrm{~Pb}$ e ${ }^{210} \mathrm{Po}$ em Bq kg-1 , intervalo de confiança, desvios padrão relativo e erros relativo. 73

TABELA 4.11 - Valores médios, mínimos e máximos de concentração dos elementos $\mathrm{Ca}$, Fe, $\mathrm{K}$ e $\mathrm{Na}(\%)$ nos derivados da Nicotiana tabacum L., pela técnica de INAA. 
TABELA 4.12 - Concentração do elemento Se $\left(\mathrm{mg} \mathrm{kg}^{-1}\right)$ nas amostras de fumo de corda e rapé pela técnica de INAA.

TABELA 4.13 - Valores médios, mínimos e máximos de concentração dos elementos traço As, Ba, Br, Co, Cr, Cs, Hf, Rb e $\mathrm{Zn}\left(\mathrm{mg} \mathrm{kg}^{-1}\right)$ nos derivados da Nicotiana tabacum L., pela técnica de INAA

TABELA 4.14 - Concentração do elemento Th $\left(\mathrm{mg} \mathrm{kg}^{-1}\right)$ nas amostras de cigarro de palha, fumo de corda e rapé pela técnica de INAA.

TABELA 4.15 - Valores médios, mínimos e máximos de concentração do elemento $\mathrm{Ce}, \mathrm{La}, \mathrm{Sc}$ e Sm $\left(\mathrm{mg} \mathrm{kg}^{-1}\right)$ nos derivados da Nicotiana tabacum L., pela técnica de INAA .88

TABELA 4.16 - Concentração do elemento Eu $\left(\mathrm{mg} \mathrm{kg}^{-1}\right)$ nas amostras de cigarro de palha, fumo de corda e rapé pela técnica de INAA. .89

TABELA 4.17 - Concentração do elemento Tb $\left(\mathrm{mg} \mathrm{kg}^{-1}\right)$ nas amostras de cigarro de palha, fumo de corda e rapé pela técnica de INAA. 89

TABELA 4.18 - Concentração do elemento $\mathrm{Yb}\left(\mathrm{mg} \mathrm{kg}^{-1}\right)$ nas amostras de cigarro de palha, fumo de corda e rapé pela técnica de INAA 89

Tabela 4.19 - Intervalo de concentração de elementos terras raras, $\mathrm{mg} \mathrm{kg}^{-1}$, presente trabalho e de Kabata-Pendias (2010). 92

Tabela 4.20 - Concentrações médias em mg kg-1 (*\%) no presente trabalho e de cigarros egípcios pela técnica INAA. . .93

Tabela 4.21 - Valores médios, mínimos e máximos de concentração dos elementos $\mathrm{Al}, \mathrm{Ca}, \mathrm{Cl}, \mathrm{Fe}, \mathrm{K}, \mathrm{I}, \mathrm{Mg}, \mathrm{Mn}, \mathrm{P}$ e $\mathrm{S}$ em \% nos derivados da Nicotiana tabacum L., pela técnica de EDXRF. .94

Tabela 4.22 - Valores médios, mínimos e máximos de concentração dos elementos traço As, Br, Cd, Co, Cr, Cs, Cu, Hg, Ni, Pb, Rb, Sb, Se, Sr,Th, U, V e Zn (mg kg-1) nos derivados da Nicotiana tabacum L., pela técnica de EDXRF 102

Tab. 4.23 - Valores médios, mínimos e máximos de concentração dos elementos terras raras $\mathrm{La}, \mathrm{Nd}$ e $\mathrm{Sm}\left(\mathrm{mg} \mathrm{kg}^{-1}\right)$ nos derivados da Nicotiana tabacum L., pela técnica de EDXRF. 116

TABELA 4.24 - Valores médios de concentração determinados no presente trabalho e nem cigarros mexicanos $\left(\mathrm{mg} \mathrm{kg}^{-1}\right)$. .118

TABELA 4.25 - Valores médios mínimos e máximos de concentração do radionuclídeos naturais $\left(\mathrm{mBq} \mathrm{g}^{-1}\right)$.. 120

TABELA 4.26 - Concentração dos radionuclídeos ${ }^{210} \mathrm{~Pb}$ e ${ }^{210} \mathrm{Po}\left(\mathrm{mBq} \mathrm{g} \mathrm{g}^{-1}\right)$ em amostras de charutos. .126

TABELA 4.27 - Dose anual estimada dos radionuclídeos ${ }^{210} \mathrm{~Pb}$ e ${ }^{210} \mathrm{Po}(\mu \mathrm{Sv}$ ano-1$)$ nos derivados de tabaco analisados e de artigos da literatura. 128 
TABELA 4.28 - Intervalo de concentração dos elementos determinados pela técnica de INAA em mg kg${ }^{-1}(* \%)$, nos derivados de tabaco analisados 130

TABELA 4.29 - intervalo de concentração dos elementos determinados pela técnica de EDXRF em $\mathrm{mg} \mathrm{kg}^{-1}\left({ }^{*} \%\right)$, nos derivados de tabaco analisados .......131

TABELA 4.29 - intervalo de concentração dos radionuclídeos naturais determinados em $\mathrm{mBq} \mathrm{g}^{-1}$, nos derivados de tabaco analisados 


\section{LISTA DE FIGURAS}

Página

FIGURA 1.1 - Registro de um europeu fumando cachimbo em 1594 .22

FIGURA 1.2 - Ilustração da colheita do tabaco. .23

FIGURA 1.3 - Ramos de tabaco no Brasão das Armas da República. 24

FIGURA 1.4 - Advertência sobre os malefícios dos cigarros. .26

FIGURA 1.5 - Advertência sobre a fumaça toxica dos cigarros. .27

FIGURA 1.6 - Série de decaimento natural do ${ }^{238} \mathrm{U}$ 31

FIGURA 1.7 - Série de decaimento natural do ${ }^{232} \mathrm{Th}$ .32

FIGURA 3.1 - Exemplos de derivados de tabaco .38

FIGURA 3.2 - amostras secas e maceradas. .39

FIGURA 3.3 - Amostras acondicionadas para irradiação. .40

FIGURA 3.4 - Detector germânio hiperpuro (HPGe). .40

FIGURA 3.5 - Espectro obtido na análise multielementar por INAA. 41

FIGURA 3.6 - Amostras de derivados de tabaco pesadas. 45

FIGURA 3.7 - Amostras em processo de dissolução e eliminação da matéria orgânica.

FIGURA 3.8 - Precipitado de $\mathrm{Ba}(\mathrm{Ra}) \mathrm{SO}_{4}$ .47

FIGURA 3.9 - Precipitado de $\mathrm{PbCrO}_{4}$ 49

FIGURA 3.10 - Detector proporcional de fluxo gasoso de baixa radiação de fundo

FIGURA 3.11 - Deposição espontânea de ${ }^{209} \mathrm{Po}$ e ${ }^{210} \mathrm{Po}$ sob agitação. 51

FIGURA 3.12 - ${ }^{209} \mathrm{Po}$ e ${ }^{210} \mathrm{Po}$ depositados 51

FIGURA 3.13 - Espectrômetro alfa .52

FIGURA 3.14 - Espectro obtido na análise de ${ }^{210} \mathrm{Po}$ .52

FIGURA 4.1 - Z score material de referência Soil 1 pela INAA .58

FIGURA 4.2 - Z score material de referência Montanna II pela INAA .58

FIGURA 4.3 - Z score material de referência Tomato Leaves pela INAA .58

FIGURA 4.4 - Z-score material de referência Peach Leaves pela técnica EDXRF.. 
FIGURA 4.5 - Z-score material de referência Sargasso pela técnica EDXRF.

FIGURA 4.6 - Z-score material de referência Tomato Leaves pela técnica EDXRF.

FIGURA 4.7 - Variação da concentração do elemento Ca (\%) nos derivados da Nicotiana tabacum $\mathrm{L}$. pela técnica de INAA.

FIGURA 4.8 - Variação da concentração do elemento K (\%) nos derivados da Nicotiana tabacum $\mathrm{L}$. pela técnica de INAA.

FIGURA 4.9 - Variação da concentração do elemento Fe (\%) nos derivados da Nicotiana tabacum L. pela técnica de INAA.

FIGURA 4.10 - Variação da concentração do elemento $\mathrm{Na}$ (\%) nos derivados da Nicotiana tabacum $\mathrm{L}$. pela técnica de INAA. .79

FIGURA 4.11 - Variação da concentração do elemento As $\left(\mathrm{mg} \mathrm{kg}^{-1}\right)$ nos derivados da Nicotiana tabacum L. pela técnica de INAA.

FIGURA 4.12 - Variação da concentração do elemento Ba $\left(\mathrm{mg} \mathrm{kg}^{-1}\right)$ nos derivados da Nicotiana tabacum $\mathrm{L}$. pela técnica INAA.

FIGURA 4.13 - Variação da concentração do elemento $\mathrm{Br}\left(\mathrm{mg} \mathrm{kg}^{-1}\right)$ nos derivados da Nicotiana tabacum L. pela técnica INAA.

FIGURA 4.14 - Variação da concentração do elemento Co $\left(\mathrm{mg} \mathrm{kg}^{-1}\right)$ nos derivados da Nicotiana tabacum L. pela técnica INAA.

FIGURA 4.15 - Variação da concentração do elemento $\mathrm{Cr}\left(\mathrm{mg} \mathrm{kg}^{-1}\right)$ nos derivados da Nicotiana tabacum L. pela técnica INAA.

FIGURA 4.16 - Variação da concentração do elemento Cs $\left(\mathrm{mg} \mathrm{kg}^{-1}\right)$ nos derivados da Nicotiana tabacum L. pela técnica INAA.

FIGURA 4.17 - Variação da concentração do elemento $\mathrm{Hf}\left(\mathrm{mg} \mathrm{kg}^{-1}\right)$ nos derivados da Nicotiana tabacum L. pela técnica INAA. 85

FIGURA 4.18 - Variação da concentração do elemento Rb $\left(\mathrm{mg} \mathrm{kg}^{-1}\right)$ nos derivados da Nicotiana tabacum L. pela técnica INAA.

FIGURA 4.19 - Variação da concentração do elemento Zn $\left(\mathrm{mg} \mathrm{kg}^{-1}\right)$ nos derivados da Nicotiana tabacum $\mathrm{L}$. pela técnica INAA.....

FIGURA 4.20 - Variação da concentração do elemento Th $\left(\mathrm{mg} \mathrm{kg}^{-1}\right)$ nos derivados da Nicotiana tabacum L. pela técnica INAA.

FIGURA 4.21 - Variação da concentração do elemento Ce $\left(\mathrm{mg} \mathrm{kg}^{-1}\right)$ nos derivados da Nicotiana tabacum $\mathrm{L}$. pela técnica INAA.

FIGURA 4.22 - Variação da concentração do elemento La ( $\left.\mathrm{mg} \mathrm{kg}^{-1}\right)$ nos derivados da Nicotiana tabacum L. pela técnica INAA. 
FIGURA 4.23 - Variação da concentração do elemento Sc $\left(\mathrm{mg} \mathrm{kg}^{-1}\right)$ nos derivados da Nicotiana tabacum L. pela técnica INAA.

FIGURA 4.24 - Variação da concentração do elemento Sm $\left(\mathrm{mg} \mathrm{kg}^{-1}\right)$ nos derivados da Nicotiana tabacum L. pela técnica INAA

FIGURA 4.25 - Variação da concentração do elemento AI (\%) nos derivados da Nicotiana tabacum L. pela técnica EDXRF. .95

FIGURA 4.26 - Variação da concentração do elemento Ca (\%) nos derivados da Nicotiana tabacum L. pela técnica EDXRF.....

FIGURA 4.27 - Variação da concentração do elemento $\mathrm{Cl}(\%)$ nos derivados da Nicotiana tabacum L. pela técnica EDXRF

FIGURA 4.28 - Variação da concentração do elemento Fe (\%) nos derivados da Nicotiana tabacum L. pela técnica EDXRF.

FIGURA 4.29 - Variação da concentração do elemento I (\%) nos derivados da Nicotiana tabacum $\mathrm{L}$. pela técnica EDXRF

FIGURA 4.30 - Variação da concentração do elemento K (\%) nos derivados da Nicotiana tabacum L. pela técnica EDXRF 98

FIGURA 4.31 - Variação da concentração do elemento Mg (\%) nos derivados da Nicotiana tabacum L. pela técnica EDXRF 99

FIGURA 4.32 - Variação da concentração do elemento Mn (\%) nos derivados da Nicotiana tabacum L. pela técnica EDXRF 100

FIGURA 4.33 - Variação da concentração do elemento P (\%) nos derivados da Nicotiana tabacum L. pela técnica EDXRF.

FIGURA 4.34 - Variação da concentração do elemento S (\%) nos derivados da Nicotiana tabacum L. pela técnica EDXRF 101

FIGURA 4.35 - Variação da concentração do elemento $P\left(\mathrm{mg} \mathrm{kg}^{-1}\right)$ nos derivados da Nicotiana tabacum $\mathrm{L}$. pela técnica EDXRF. 104

FIGURA 4.36 - Variação da concentração do elemento $\mathrm{Br}\left(\mathrm{mg} \mathrm{kg}^{-1}\right)$ nos derivados da Nicotiana tabacum L. pela técnica EDXRF. 105

FIGURA 4.37 - Variação da concentração do elemento Cd $\left(\mathrm{mg} \mathrm{kg}^{-1}\right)$ nos derivados da Nicotiana tabacum L. pela técnica EDXRF 106

FIGURA 4.38 - Variação da concentração do elemento Co $\left(\mathrm{mg} \mathrm{kg}^{-1}\right)$ nos derivados da Nicotiana tabacum L. pela técnica EDXRF. 106

FIGURA 4.39 - Variação da concentração do elemento $\mathrm{Cr}\left(\mathrm{mg} \mathrm{kg}^{-1}\right)$ nos derivados da Nicotiana tabacum L. pela técnica EDXRF. 107

FIGURA 4.40 - Variação da concentração do elemento Cs $\left(\mathrm{mg} \mathrm{kg}^{-1}\right)$ nos derivados da Nicotiana tabacum L. pela técnica EDXRF 
FIGURA 4.41 - Variação da concentração do elemento Cu $\left(\mathrm{mg} \mathrm{kg}^{-1}\right)$ nos derivados da Nicotiana tabacum L. pela técnica EDXRF.

FIGURA 4.42 - Variação da concentração do elemento $\mathrm{Hg}\left(\mathrm{mg} \mathrm{kg}^{-1}\right)$ nos derivados da Nicotiana tabacum L. pela técnica EDXRF

FIGURA 4.43 - Variação da concentração do elemento Hg (mg kg-1) nos derivados da Nicotiana tabacum L. pela técnica EDXRF

FIGURA 4.44 - Variação da concentração do elemento $\mathrm{Hg}\left(\mathrm{mg} \mathrm{kg}^{-1}\right)$ nos derivados da Nicotiana tabacum L. pela técnica EDXRF

FIGURA 4.45 - Variação da concentração do elemento Rb $\left(\mathrm{mg} \mathrm{kg}^{-1}\right)$ nos derivados da Nicotiana tabacum L. pela técnica EDXRF

FIGURA 4.46 - Variação da concentração do elemento Sb $\left(\mathrm{mg} \mathrm{kg}^{-1}\right)$ nos derivados da Nicotiana tabacum L. pela técnica EDXRF

FIGURA 4.47- Variação da concentração do elemento Se $\left(\mathrm{mg} \mathrm{kg}^{-1}\right)$ nos derivados da Nicotiana tabacum L. pela técnica EDXRF.

FIGURA 4.48 - Variação da concentração do elemento Sr $\left(\mathrm{mg} \mathrm{kg}^{-1}\right)$ nos derivados da Nicotiana tabacum L. pela técnica EDXRF.

FIGURA 4.49 - Variação da concentração do elemento Th $\left(\mathrm{mg} \mathrm{kg}^{-1}\right)$ nos derivados da Nicotiana tabacum L. pela técnica EDXRF

FIGURA 4.50 - Variação da concentração do elemento $U\left(\mathrm{mg} \mathrm{kg}^{-1}\right)$ nos derivados da Nicotiana tabacum L. pela técnica EDXRF

FIGURA 4.51 - Variação da concentração do elemento $\mathrm{V}\left(\mathrm{mg} \mathrm{kg}^{-1}\right)$ nos derivados da Nicotiana tabacum L. pela técnica EDXRF.

FIGURA 4.52 - Variação da concentração do elemento Zn $\left(\mathrm{mg} \mathrm{kg}^{-1}\right)$ nos derivados da Nicotiana tabacum L. pela técnica EDXRF

FIGURA 4.53 - Variação da concentração do elemento La $\left(\mathrm{mg} \mathrm{kg}^{-1}\right)$ nos derivados da Nicotiana tabacum L. pela técnica EDXRF.

FIGURA 4.54 - Variação da concentração do elemento $\mathrm{Nd}\left(\mathrm{mg} \mathrm{kg}^{-1}\right)$ nos derivados da Nicotiana tabacum L. pela técnica EDXRF

FIGURA 4.55 - Variação da concentração do elemento Sm $\left(\mathrm{mg} \mathrm{kg}^{-1}\right)$ nos derivados da Nicotiana tabacum L. pela técnica EDXRF

FIGURA 4.56 - Variação da concentração do radionuclídeo ${ }^{226} \mathrm{Ra}\left(\mathrm{mBq} \mathrm{g}{ }^{-1}\right)$ nos derivados da Nicotiana tabacum L.

FIGURA 4.57 - Variação da concentração do radionuclídeo ${ }^{210} \mathrm{~Pb}\left(\mathrm{mBq} \mathrm{g}^{-1}\right)$ nos derivados da Nicotiana tabacum L.

FIGURA 4.58 - Variação da concentração do radionuclídeo ${ }^{210} \mathrm{Po}\left(\mathrm{mBq} \mathrm{g}^{-1}\right)$ nos derivados da Nicotiana tabacum L. 
FIGURA 4.59 - Variação da concentração do radionuclídeo ${ }^{238} \mathrm{U}\left(\mathrm{mBq} \mathrm{g}^{-1}\right)$ nos derivados da Nicotiana tabacum L.

FIGURA 4.60 - Variação da concentração do radionuclídeo 228Ra $\left(\mathrm{mBq} \mathrm{g}^{-1}\right)$ nos derivados da Nicotiana tabacum L.

FIGURA 4.61 - Variação da concentração do radionuclídeo 232Th $\left(\mathrm{mBq} \mathrm{g}^{-1}\right)$ nos



FIGURA 4.62 - Concentração média dos radionuclídeos em mBq cigarro-1.....124

FIGURA 4.63 - Concentração dos radionuclídeos ${ }^{226} \mathrm{Ra},{ }^{210} \mathrm{~Pb}$ e ${ }^{210} \mathrm{Po}$ ( $\mathrm{mBq}$ cigarro ${ }^{-1}$ ) em amostras de cigarros: presente trabalho e dados da literatura

FIGURA 4.64 - Dose média estimada de ${ }^{210} \mathrm{~Pb}$ e ${ }^{210} \mathrm{Po}\left(\mu \mathrm{Sv}_{\text {ano }}{ }^{-1}\right)$ nos derivados de tabaco analisados

FIGURA 4.65 - Dose média estimada de ${ }^{210} \mathrm{~Pb}$ e ${ }^{210} \mathrm{Po}(\mu \mathrm{Sv}$ ano-1 $)$ em amostras de cigarro do presente trabalho e da literatura ................................................127

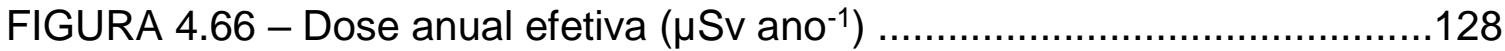




\section{INTRODUÇÃO}

O levantamento sobre a população tabagista brasileira realizada pelo IBGE (Instituto Brasileiro de Geografia e Estatística) em 2013 constatou que 21,9 milhões de pessoas fazem uso de produtos derivados do tabaco, fumado ou não fumado, de uso diário ou ocasional, sendo a forma mais consumida o cigarro industrializado. Esse número representa $15 \%$ de toda a população brasileira, número bastante significativo considerando que os tabagismo é um dos principais fatores de risco evitáveis à saúde. Morrem aproximadamente 7 milhões de pessoas todo ano de causa diretamente relacionada ao consumo de tabaco. O tabaco gera um custo superior a US\$1,4 trilhões em cuidados de saúde e perda de produtividade no mundo (WHO, 2017).

O tabaco é um insumo conhecido no território brasileiro muito antes da chegada dos portugueses; originário dos Andes, o tabaco chegou ao Brasil pela migração dos índios no Continente Americano (SOUZA CRUZ, 2016; SINDITABACO,2016). O cultivo da planta no território nacional começou antes mesmo da colonização portuguesa pelas tribos indígenas (SINDITABACO,2016). As folhas da planta eram usadas pelos indígenas em rituais e cerimonias religiosas na busca de uma leveza xamânica que permitia o alcance de um estado de sonho onde era possível interação com os espíritos e mortos; porém, somente era usado pelos grandes pajés (AVELAR, 2010).

Na história pré-colonial um outro uso para o tabaco era o medicinal, para cura de ferimentos, enxaquecas e dores de estômago, sendo que este podia ser consumido de diversas formas: mascado, aspirado, bebido e fumado (SINDITABACO, 2016).

Também conhecido como petyn (no tupi) ou ainda betum, petema, peti, petigma, petima, petume, pitima, pituma, potum e petum, o tabaco foi documentado em diversas incursões europeias ao território brasileiro em meados do século XVI. Estes o relatavam como uma erva fascinante gerando admiração e encanto aos que a conheciam (MELLO Jr e KOCKEL, 2010).

Aos poucos o tabaco deixou de ser uma planta cultivada somente pelos indígenas para rituais religiosos e passou a ser um dos principais produto 
comerciais destinado à Europa durante 0 período imperial brasileiro (SINDITABACO, 2016).

As primeiras plantações de tabaco pelos colonos portugueses eram destinadas ao consumo próprio (BONATO, 2006), mas a medida em que europeus experimentavam a erva, seu consumo e procura aumentava e após um século passou a ser conhecido e consumido no mundo inteiro. O tabaco era considerado um bom passatempo (SINDITABACO, 2016). A FIG.1.1 apresenta a ilustração de um europeu fumando cachimbo.

FIGURA 1.1 - Registro de um europeu fumando cachimbo em 1594 (www.ajaraujo.com.br)

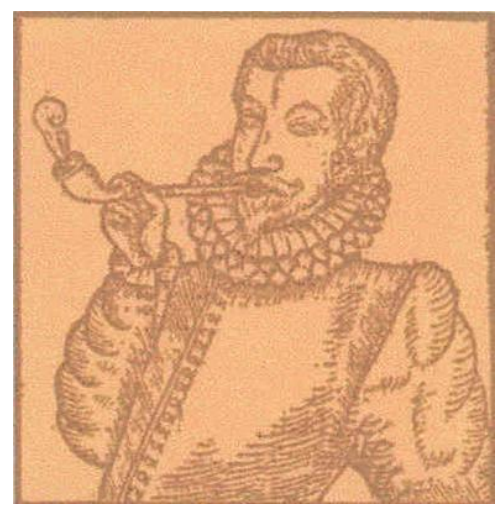

Mudas da planta de tabaco também foram levadas para a Europa pelas expedições portuguesas difundindo o uso para muitos países da Europa, África e do Oriente (SINDITABACO, 2016) e se tornou um importante produto oferecido pela Companhia das Índias Ocidentais (BONATO, 2006).

O cultivo comercial do tabaco no Brasil, iniciou-se na Bahia com a finalidade de produto de trocas comerciais com a Europa, garantindo a mão-deobra escrava para o cultivo e ao mesmo tempo o beneficiamento do açúcar (SILVEIRA, DORNELLES e FERRARI, 2012); este cultivo foi crescendo à medida em que se intensificava a necessidade de escravos. Pacotes de folhas secas ou rolos viravam moedas de troca com navios negreiros da Guiné e Angola durante o século XIX (CAETANO, 2003, MARQUES, 2011). A FIG. 1.2 é uma ilustração da colheita de folhas de tabaco no estado da Bahia. 
FIGURA 1.2 - Ilustração da colheita do tabaco (http://www.brasilcult.pro.br)

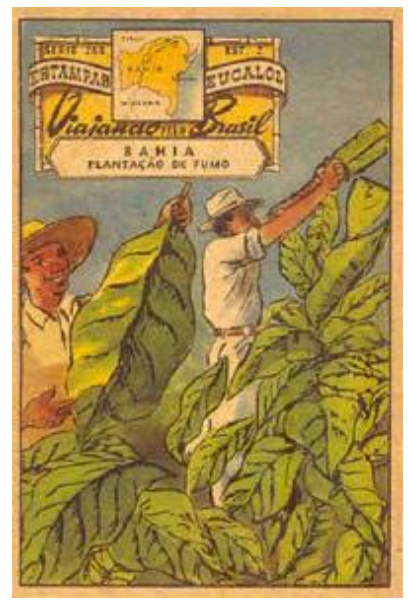

Em 1674 foi criada a Junta de Administração do Tabaco com a finalidade legislativa e reguladora para a produção do tabaco, dando início à uma pesada tributação ao insumo. Neste período o tabaco nacional tinha três destinos: exportação do produto considerado de primeira e segunda qualidade para Lisboa, aproximadamente $60 \%$ da produção, e esta reexportada para os demais mercados consumidores da Europa; uma parte da classificada como de terceira qualidade era negociada com navios negreiros e o restante destinava-se ao consumo local (BONATO,2006).

O tabaco e a aguardente eram consideradas produtos vitais no cotidiano escravagista, eram vistos como substâncias suavizadoras das árduas funções exercidas pelos escravos (AVELAR, 2010).

Após 1810 com a abertura dos portos às nações amigas do Brasil o cultivo da planta se desconcentrou e a parir de 1850 iniciou-se a produção nos estados de Minas Gerais, Rio Grande do Sul e Santa Catarina (SOUZA CRUZ, 2016; SINDITABACO, 2016).

A importância do tabaco está destacada também no brasão das Armas da República do Brasil que possui ramos de tabaco, FIG. 1.3 (SINDITABACO, 2016). 
FIGURA 1.3 - Ramos de tabaco no Brasão das Armas da República (Www2.planalto.gov.br)

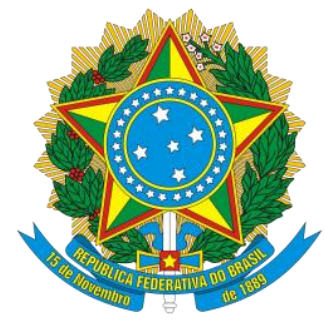

No cenário atual, o Brasil é o segundo produtor mundial de tabaco e desde 1993 destaca-se como o maior exportador oferecendo produto de alta qualidade. Na safra 2014/15 foram registradas 692 mil toneladas de tabaco e $85 \%$ foram destinados ao mercado externo; 97 países consomem o tabaco brasileiro, sendo os principais compradores a União Europeia com 43\%, seguida pelo Extremo Oriente (25\%) e América do Norte (11\%) (SINDITABACO, 2016).

O cultivo do tabaco representa uma importante atividade agrícola principalmente para os estados do Sul do Brasil que produz 95\% do volume total de tabaco, distribuído em três estados - 51\% no Rio Grande do Sul, 29\% em Santa Catarina e $20 \%$ no Paraná. Os 5\% restantes são cultivados nos Estados da Bahia e Alagoas, onde são encontradas as fábricas de charuto. O tabaco representou, em $2015,1,14 \%$ do total das exportações com um faturamento de mais de US\$2,19 bilhões (SINDITABACO, 2016).

A industrialização do tabaco introduziu no mercado novos produtos e derivados mais baratos tornando-os acessíveis a população de baixa renda. Outro grande popularizador do consumo do tabaco foram as guerras onde os cigarros eram sempre vistos nas mãos dos soldados (BONATO, 2006).

Outro grande responsável pela popularização dos derivados de tabaco, principalmente o cigarro a partir da década de 60 foi o setor cinematográfico, com filmes e propagandas que transformaram o ato de fumar em um fenômeno da moda (BONATO, 2006).

\subsection{Tabagismo: questão de saúde pública}

Considerado como uma doença neurocomportamental o tabagismo está associado a diversos distúrbios como ansiedade, depressão, problemas 
psiquiátricos e baixa autoestima, além dos fatores culturais e genéticos que induzem ao ato de fumar. Não é um estilo de vida, charme, fator de sociabilidade ou expressão de livre arbítrio, as pessoas fumam não porque querem mas porque são dependentes (SILVA et al, 2016).

O tabagismo geralmente se inicia ainda na adolescência, antes de desenvolver plenamente o conhecimento sobre o poder viciante, as questões de saúde e mortalidade associados ao tabaco (HECHT, 2012).

O tabaco é a segunda droga mais consumida entre os estudantes brasileiros e segundo o próprio Governo é considerado uma importante porta de entrada para o uso das drogas ilícitas (INCA, 2011).

O uso de tabaco é uma das principais causas de doença e morte prematuras no mundo (HARRIS e ANTHENELLI, 2005), contribuindo para uma parcela considerável $(4,1 \%)$ da carga global de doenças e vem aumentando rapidamente em países em desenvolvimento e entre as mulheres.

A principal malignidade associada ao consumo de tabaco é o câncer de pulmão, aproximadamente 90\% dos casos. Estima-se que em 2016 foram diagnosticados 17.330 novos casos em homens e 10.890 em mulheres, e aproximadamente um 1.700 .000 novos casos por ano no planeta, causando 1.400.000 mortes por ano (A.C.CAMARGO, 2017).

Além do câncer de pulmão o consumo do tabaco também está relacionado a diversos outros tipos de câncer, como o de colo de útero, da cavidade oral, de esôfago, de bexiga e o de laringe; e está associado também a doenças respiratórias e cardiovasculares. A incidência dessas doenças está relacionada ao tempo do hábito de fumar e com a quantidade de cigarros fumados por dia (INCA, 2017).

O tabagismo é responsável por $10 \%$ dos óbitos globais, sendo considerado a principal causa previsível de morbidade e mortalidade no mundo, gerando importante custo aos cofres públicos com consultas, hospitalizações, exames, procedimentos de alta complexidade e recursos humanos (PINTO e UGÁ, 2011). 
Em 2001, por determinação do Governo brasileiro, foram incluídas nas embalagens de produtos de tabaco advertências sanitárias informando os malefícios do consumo de tabaco e o número de telefone do Disque Saúde - Pare de Fumar, como apresentado na FIG 1.4.

FIGURA 1.4 - Advertência sobre os malefícios dos cigarros. (www2.inca.gov.br)
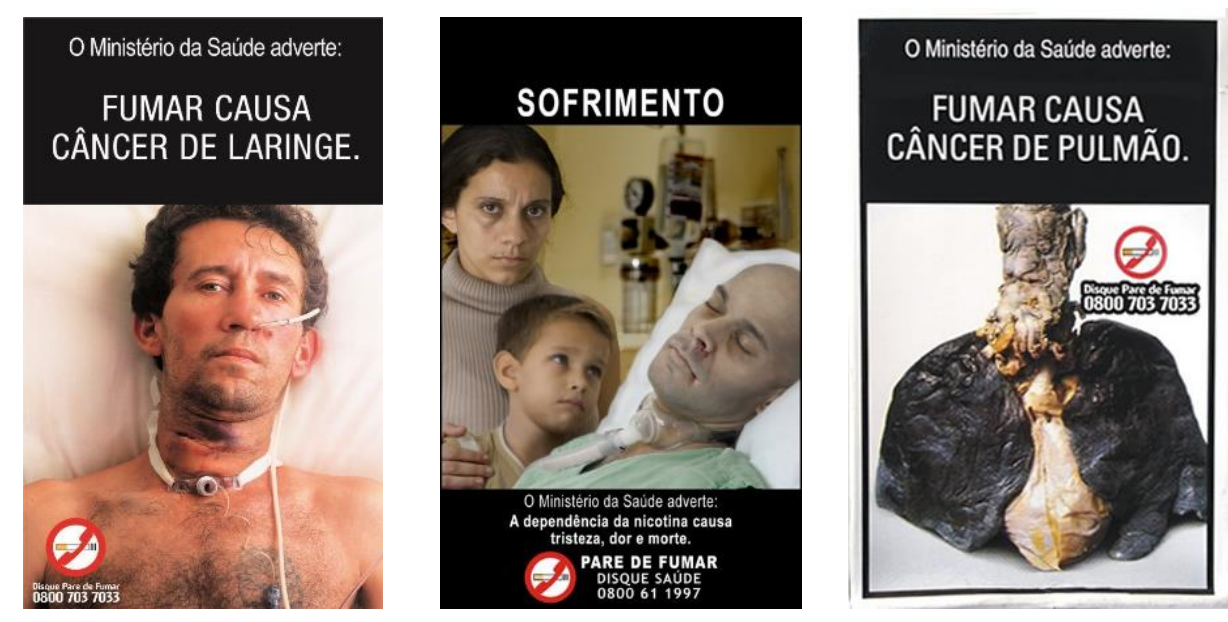

Sabe-se que na fumaça do cigarro existem mais de 5.000 compostos químicos, sendo alguns agentes tóxicos, irritantes, inflamatórios e cocarcinogênicos, incluindo 73 compostos carcinogênicos (HECHT, 2012) e que na planta de tabaco existem metais tóxicos ao organismo humano, provenientes da absorção do solo, fertilizantes e pesticidas, e que no processo de industrialização do cigarro esses elementos podem ter suas concentrações aumentadas (TALIO, LUCONI e FERNANDEZ, 2011). A FIG. 1.5 é a imagem de advertência sobre a toxidade da fumaça dos cigarros usadas nas campanhas de conscientização do Ministério da Saúde.

Mais de 20 substâncias presentes na fumaça do cigarro são conhecidas como carcinogênicas pulmonares, dentre elas estão os hidrocarbonetos aromáticos voláteis, metais como o cádmio e elementos químicos radioativos como o ${ }^{210} \mathrm{Po}$. A nicotina é o principal composto químico responsável pela dependência do tabagista, mas não é considerado um causador de câncer de pulmão (HECHT, 2012). 
FIGURA 1.5 - Advertência sobre a fumaça toxica dos cigarros. (www2.inca.gov.br)

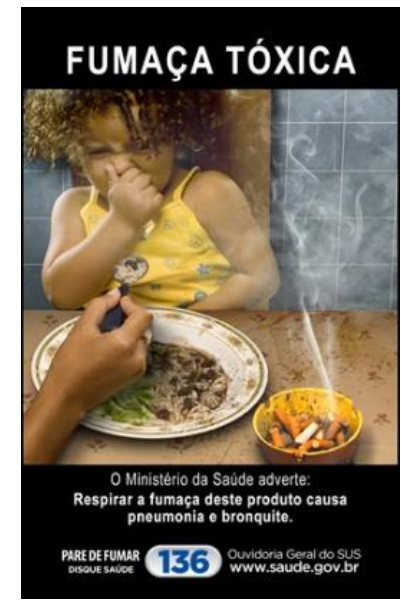

Os efeitos nocivos do tabaco ultrapassam as gerações, aumentando os riscos de doenças respiratórias nas gerações futuras em até 3 vezes, mesmo que gerações intermediárias não tenham sido tabagistas; o não tabagista tem melhor qualidade de vida e vive 10 a 15 anos a mais (SILVA et al, 2016).

ZAGA, et al. (2011) cita que até a segunda metade do século XX o câncer de pulmão era considerado uma doença extremamente rara. Após esses período a doença teve um aumento tão rápido que passou a ser considerada uma epidemia em homens de países desenvolvidos; nos países que adotaram alguma medida de controle da dependência do tabaco nos anos cinquenta, a incidência de câncer de pulmão reduziu.

Os fumantes passivos também são de grande preocupação pois como estão expostos à fumaça do tabaco onde vivem ou trabalham também estão propensos a desenvolver câncer de pulmão, devido a inalação da corrente secundária produzida na combustão dos produtos de tabaco, sendo esta uma importante fonte de poluição ambiental (WÜNSCH FILHO, 2010).

A poluição tabagística ambiental é formada pela fumaça inalada pelo fumante, denominada corrente primaria e a fumaça que sai da ponta do cigarro, denominada corrente secundaria, sendo esta última corresponde a $96 \%$ do tempo total da queima dos derivados do tabaco (INCA, 2017). 
Segundo informações do Ministério da Saúde (INCA, 2017) a fumaça inalada pelo não-fumante tem oito vezes mais monóxido de carbono, três vezes mais nicotina e até cinquenta vezes mais substancias cancerígenas que a fumaça tragada pelo fumante.

Um estudo realizado com 400 amostras necrópsicas de mulheres não tabagistas que não tiveram mortes relacionadas à doenças respiratórias ou câncer, apresentou resultados com valores significantemente mais elevados de lesões précancerosas no epitélio pulmonar em mulheres com cônjuges tabagistas do que as casadas com não fumantes (TRICHOPOULOS, 1992).

\subsection{A toxidade dos derivados do tabaco}

Diversos trabalhos relatam a toxidade dos derivados do tabaco, dentre eles alguns estudos prévios realizados no Brasil avaliaram e quantificam a presença de elementos tóxicos ao ser humano em amostras de cigarro brasileiro, como o trabalho de PERES (1999) que estudou a presença de ${ }^{210} \mathrm{~Pb}$ e ${ }^{210} \mathrm{Po}$, o de FRANCISCONI et. al. (2011) que determinaram a presença de As, Se, Sb e Cr; e o de SILVA et al. (2013) que determinaram os teores de $\mathrm{Cd}, \mathrm{Hg}$ e $\mathrm{Pb}$.

EXLEY et al. (2006) verificaram elevados teores de alumínio em amostras de tabaco jamaicano. Os autores explicaram que o Al é volatilizado durante a queima do tabaco e entra nos pulmões na forma de partículas da porção gasosa da fumaça. Durante o processo de queima, o Al pode formar complexos gasosos que são rapidamente dissolvidos nas membranas celulares e entram assim no sistema orgânico e é eliminado pela urina. O Al particulado retido nos pulmões apresenta grande potencial de indução de reações inflamatórias. Por ação mucociliar este é removido dos pulmões para o intestino e posteriormente expelido do organismo, explicando assim o porquê de tabagistas apresentarem teores mais elevados de Al na urina do que os não fumantes.

PANTA et al. (2008) analisaram o conteúdo de Hg presente em amostras de cigarros, de charutos e de tabaco de mascar norte americanos e verificaram que os charutos apresentaram quantidade significativamente maiores que os cigarros e o tabaco de mascar. Os autores citam que a fumaça do cigarro é uma importante fonte de $\mathrm{Hg}$ devido à volatilidade do metal e que as concentrações do elemento 
variaram com o tipo, local e forma como a planta de tabaco foi cultivada. A análise de $\mathrm{Hg}$ em produtos de tabaco é importante para a saúde humana e monitoramento ambiental.

PAPPAS (2011) fez um levantamento de diversos metais e metaloides tóxicos e ou cancerígenos presentes nos cigarros e derivados de tabaco não fumados, dentre eles o Al, As, Ba, Be, Cd, Cr, Cu, Fe, Pb, Mn, Hg, Ni, Co, e Si, podendo ser encontrados na forma iônica ou gasosa elementar. O autor ressalta o estudo de produtos de tabaco são importantes pois conferem riscos à saúde.

MOHAPATRA et al. (2014) em um estudo de metais pesados em cigarros indianos, constataram que o tabagismo é um contribuinte no desenvolvimento do câncer de mama. Esse estudo demonstrou a carcinogenidade do Cd, um metal de grande citotoxidade e capaz de transformar células epiteliais normais em células potencialmente carcinogênicas. Os autores citam também que além do $\mathrm{Cd} \mathrm{o} \mathrm{Hg}, \mathrm{Cr}, \mathrm{Cu}$ e $\mathrm{Zn}$ estão presentes no tabaco e também são considerados metais citotóxicos.

ELDRIDGE et al. (2015) estudaram a variabilidade de compostos tóxicos de três tipos de cigarros com o propósito de entender a representatividade ao longo de um período de tempo, e dentre os compostos tóxicos estudados estavam os metais Se, As, Hg, Cd, Ni, Cr, e Pb. Verificaram que o coeficiente de variação da concentração ficou abaixo de $15 \%$ para a maioria dos analitos estudados. Os elementos $\mathrm{Hg}$ e Se tiveram concentrações abaixo de 0,031 $\mu \mathrm{g} / \mathrm{g}$.

MILNEROWICZ et al. (2015) cita que na fumaça do tabaco existem mais de trinta metais, dentre eles o $\mathrm{Al}, \mathrm{As}, \mathrm{Cd}, \mathrm{Hg}$, Ni e Pb que são capazes de interferir nos processos celulares, nas reações bioquímicas e na homeostase celular, devido principalmente à suas capacidades de gerar radicais livres que promovem um desequilíbrio pro-oxidante/ antioxidante, induzindo assim uma resposta inflamatória no organismo.

O mercúrio é um dos metais pesados mais tóxicos presentes no meio ambiente e de fácil absorção como vapor pelos pulmões, penetrando facilmente pela barreira hematoencefálica e barreira placentária podendo causar danos ao 
sistema nervoso central. O Hg também pode causar um desequilíbrio de cálcio celular, danificar mitocôndrias e consequentemente danos ou até a morte celular (MILNEROWICZ et al., 2015)

PIADÉ et al. (2015) analisaram o conteúdo de tabaco de 568 amostras de cigarros canadenses e segundo os autores a transferência de $\mathrm{Cd}$, As e Pb para a fumaça é consideráveis. Citam também que o peso corporal de cádmio (quantidade de cadmio acumulada no organismo) em fumantes é duas vezes maior que em não fumantes, sendo esse fato observado quando se avalia a concentração do elemento no tecido ósseo. Associam o $\mathrm{Cd}$ ao câncer de pulmão, pâncreas, mama, próstata, endométrio e bexiga urinária e a doenças não cancerígenas dos rins e ossos.

Segundo a Organização Mundial da Saúde (OMS) tabagistas são mais expostos ao As do que os não-tabagistas, pois as plantas de tabaco absorvem o As presente naturalmente no solo, mas o uso de inseticidas ricos em arseniato de chumbo elevam o potencial de exposição do tabagista ao elemento (WHO, 2017).

A exposição prolongada ao As pode causar câncer de bexiga e de pulmão, dentre outros efeitos adversos a saúde como neurotoxidade, diabetes, doenças pulmonares e cardiovasculares (WHO,2017).

Além dos elementos considerados quimicamente tóxicos a saúde existem outros elementos que são essenciais para o crescimento da planta de tabaco que podem reagir durante a combustão do cigarro formando compostos halogenados tóxicos, como o $\mathrm{Br}$ e Cl. No tabaco foram identificados 242 compostos halogenados sendo destes 192 halogenados pelo Cl e 12 pelo Br (MÜLLER et al., 2011).

\subsection{Radionuclídeos naturais no tabaco}

Os radionuclídeos naturais das séries do ${ }^{238} \mathrm{U}$ e ${ }^{232} \mathrm{Th}$, FIG. 1.6 e FIG1.7, são encontrados normalmente em baixas concentrações no tabaco provenientes do solo ou por deposição foliar (ABD EL-AZIZA, KHATERA E AL-SEWAIDAN, 2005; CHAUHAN E CHAUHAN, 2014). Desde a década de 60 alguns estudos relatam a presença de ${ }^{210} \mathrm{Po}$ e ${ }^{210} \mathrm{~Pb}$ tanto na fase gasosa como na particulada da 
fumaça de cigarros, ambos radionuclídeos filhos do ${ }^{226} \mathrm{Ra}$ e da série de decaimento natural do ${ }^{238} \mathrm{U}$, (KILTHAU, 1996), FIG. 1.6.

As plantas de tabaco absorvem esses radionuclídeos do solo durante seu crescimento, mas além do solo outra forma de absorção é a deposição foliar principalmente do ${ }^{210} \mathrm{~Pb}$ que é produto de decaimento do ${ }^{222} \mathrm{Rn}$ de ocorrência natural na atmosfera (KILTHAU, 1996; IWAOKA e YONEHARA, 2012; ZAGA, et al. 2011).

FIGURA 1.6 - Série de decaimento natural do ${ }^{238}$ U (IVANOVICH e HARMON, 1992)

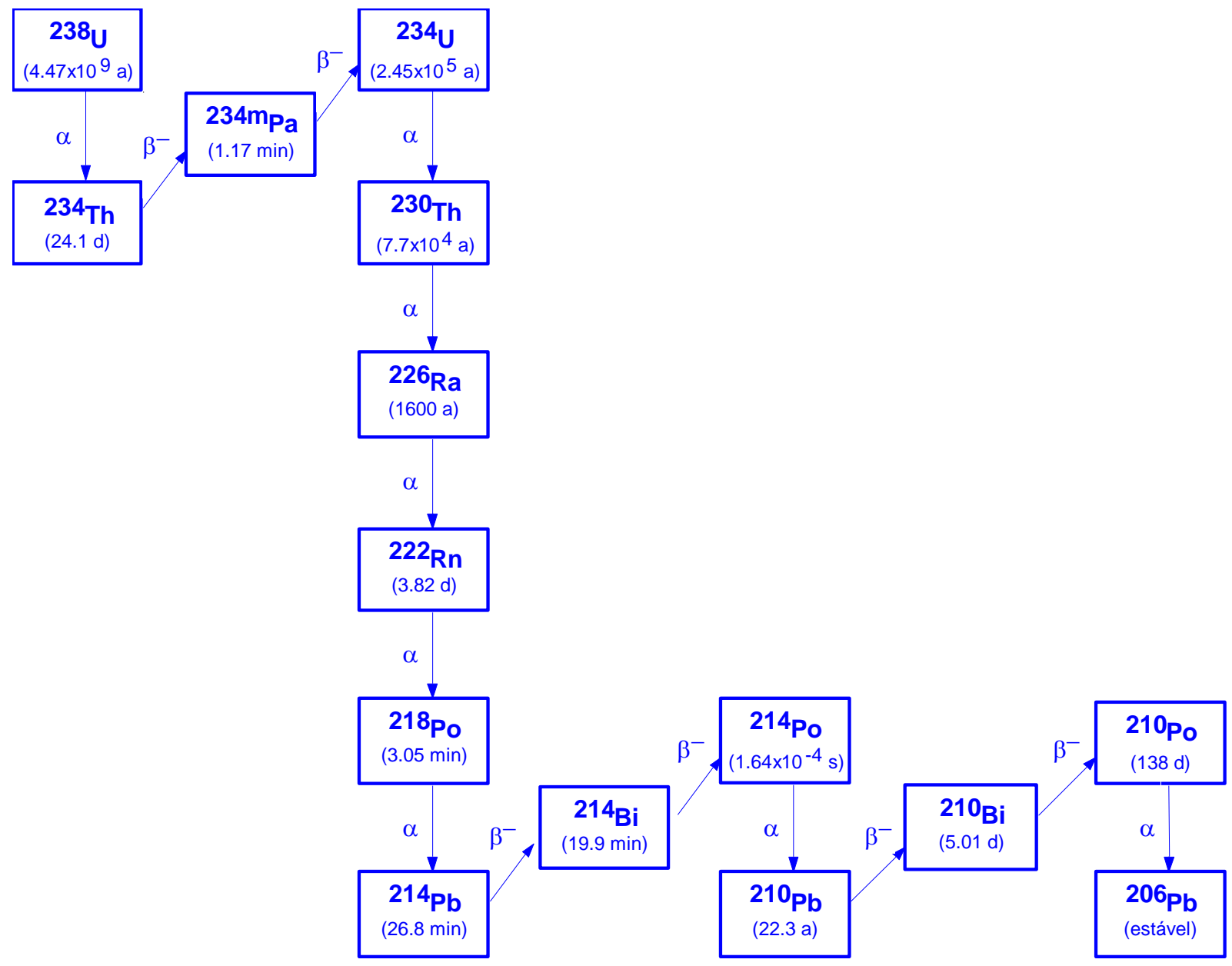


FIGURA 1.7 - Série de decaimento natural do ${ }^{232}$ Th (IVANOVICH e HARMON, 1992)

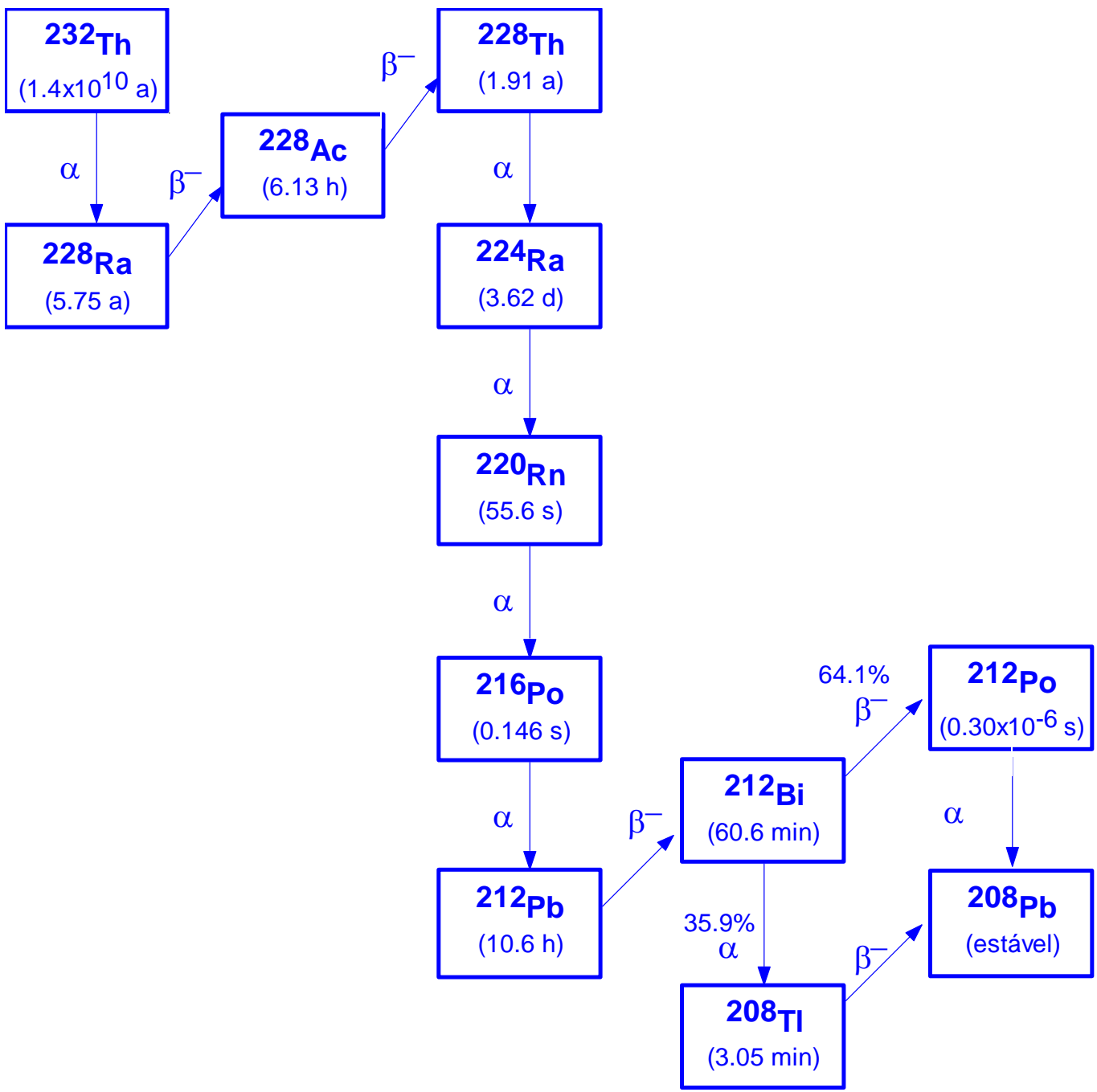

Livre na atmosfera o ${ }^{222} \mathrm{Rn}$, gás inerte, é encontrado no solo pelo decaimento do ${ }^{226} \mathrm{Ra}$ (série do ${ }^{238} \mathrm{U}$ ) e escapa para o ar. Esse gás se liga a partículas de poeira que se acumulam nas folhas de tabaco. Estas por sua vez apresentam características que facilitam a impregnação desse radionuclídeo. As folhas de tabaco apresentam projeções pegajosas, resinosas, insolúveis em água em ambos os lados fazendo com que as folhas apresentem concentrações de até 10.000 vezes maiores que a concentração nas demais partes da planta (KARAGUEUZIAN et al., 2012). O ${ }^{222} \mathrm{Rn}$ produz em seu decaimento o ${ }^{210} \mathrm{~Pb}$ que por sua vez decai para o ${ }^{210} \mathrm{Po}$, este último responsável pela maior parte da 
radioatividade dos cigarros. $\mathrm{O}^{210} \mathrm{~Pb}$ e o ${ }^{210} \mathrm{Po}$ formam complexos insolúveis com a resina das folhas de tabaco altamente resistente a lavagem por precipitação ou processo de cura das folhas de tabaco (KARAGUEUZIAN et al., 2012; ZAGA, et al., 2011).

As concentrações desses radionuclídeos presentes nas plantas de tabaco variam conforme a região do plantio e com os métodos de cultivo da planta. Plantas de tabaco indianas apresentaram cinco vezes menos ${ }^{210} \mathrm{Po}$ do que plantas norte americanas. Essas diferenças consideráveis nas concentrações dos radionuclídeos se deve as diferentes precipitações naturais, a radioatividade natural do solo e diferenças do $\mathrm{pH}$ do solo cultivado e ao tipo de fertilização usada (KILTHAU, 1996; ZAGA, et al., 2011).

No ato de fumar ocorre a transferência desses radionuclídeos, por meio da queima do tabaco, para os tecidos e órgãos humanos (KILTHAU, 1996; ABD EL-AZIZA, KHATERA E AL-SEWAIDAN, 2005; CHAUHAN E CHAUHAN, 2014). ${ }^{210} \mathrm{Po}$ e o ${ }^{210} \mathrm{~Pb}$ são volatilizados à temperatura de queima dos cigarros (SAKODA, 2012) e se depositam nos tecidos pulmonares dos tabagistas gerando dose de radiação localizada superiores as doses naturais dos não-tabagistas. Esta exposição por longos períodos aumentam significantemente o risco de indução de lesões cancerígenas (KILTHAU, 1996).

Alguns estudos verificaram que a concentração de ${ }^{210} \mathrm{Po}$ na urina de fumantes é cerca de seis vezes maior que na de não fumantes e que a taxa de câncer de bexiga aumenta consideravelmente em relação aos que não fumam. Outros estudos mostram que concentrações de ${ }^{210} \mathrm{~Pb}$ e ${ }^{210} \mathrm{Po}$ nas costelas e tecidos alveolares dos fumantes são cem vezes mais altas que nos não fumantes. $\mathrm{O}{ }^{210} \mathrm{Po}$ também pode ser encontrado nos tecidos ósseos dos tabagista devido a sua afinidade relativamente alta por este tecido, aumentando o risco de leucemia e tornando este como um marcador tumoral (KILTHAU, 1996).

Alguns fertilizantes, principalmente os fosfatados, usados nas plantações de tabaco, podem alterar a fisiologia das plantas, modificando a absorção dos minerais do solo pelas raízes das plantas e consequentemente aumentando o teor de radionuclídeos absorvidos, dentre os quais o ${ }^{226} \mathrm{Ra}$ que 
apresenta maior potencial de translocação e acúmulo na planta (CHAUHAN E CHAUHAN, 2014), e com isso, aumenta os teores do ${ }^{210} \mathrm{Po}$ e ${ }^{210} \mathrm{~Pb}$ nas folhas de tabaco (KILTHAU, 1996).

Experimentos em plantas de feijão mostram que mais de $90 \%$ do ${ }^{210} \mathrm{~Pb}$ depositado nas folhas ficam retidos na planta e aproximadamente $30 \%$ do ${ }^{210} \mathrm{Po}$, e que pequenas concentrações do ${ }^{210} \mathrm{Po}$ se transloca para outras partes da planta (ATHALYE, 1972).

Um estudo realizado com charutos brasileiros apresentou concentrações médias de ${ }^{210} \mathrm{Po}\left(26 \mathrm{mBq} \mathrm{g}{ }^{-1}\right)$ e de ${ }^{210} \mathrm{~Pb}\left(15 \mathrm{mBq} \mathrm{g}{ }^{-1}\right)$, maiores do que as encontradas em charutos cubanos ( ${ }^{210} \mathrm{Po} 5 \mathrm{mBq} \mathrm{g} \mathrm{g}^{-1}{ }^{210} \mathrm{~Pb} 2 \mathrm{mBq} \mathrm{g}{ }^{-1}$ ) e norteamericanos ( ${ }^{210} \mathrm{Po} 16,9 \mathrm{mBq} \mathrm{g}{ }^{-1} \mathrm{e}^{210} \mathrm{~Pb} 5,4 \mathrm{mBq} \mathrm{g}^{-1}$ ) (KELECON, 2002).

Os filtros comumente encontrados nos cigarros comerciais retém uma parte insignificante dos radionuclídeos, sendo praticamente toda a concentração desses encontradas na fumaça do cigarro inalada diretamente para os pulmões (KILTHAU, 1996). Cerca de $50 \%$ do ${ }^{210} \mathrm{Po}$ de um cigarro é transferido com a fumaça, 35\% permanecem na bituca e 15\% são encontrados nas cinzas (ZAGA, et al., 2011).

Segundo ZAGA, et al. (2011) citam que os níveis de ${ }^{210} \mathrm{Po}$ encontrado no sangue, urina, fígado, rim, coração, pulmão e alguns músculos e ossos chegam a ser até seis vezes maior que o de um não fumante.

Estudos realizados desde a década de 60 relatam a presença de ${ }^{210} \mathrm{Po}$ $\mathrm{e}^{210} \mathrm{~Pb}$ tanto na fase gasosa como na particulada da fumaça de cigarros (KILTHAU, 1996; KARAGUEUZIAN et al., 2012), alertando sobre o risco potencial de câncer devido a radioatividade da fumaça do cigarro. Porém, as indústrias não alertaram os consumidores sobre este potencial risco, proibiram quaisquer publicações relacionadas à radioatividade do tabaco e resistiram às pesquisas de remoção desses radionuclídeos das folhas de tabaco, argumentando desacreditar na veracidade, reprodutibilidade e confiabilidade dos dados publicados (KARAGUEUZIAN et al., 2012). 
Nos últimos anos a população brasileira vem se conscientizando dos diversos problemas de saúde causados pelo consumo de cigarros, graças às campanhas publicitárias. Porém, pouco se discute sobre o uso de outros derivados do tabaco, e dos problemas que estes também possam causar a saúde humana. Devido a estas mesmas campanhas publicitárias alertando sobre os perigos de cigarro, alguns derivados do tabaco, como narguilé ou tabaco de mascar, tiveram seu consumo aumentado, principalmente entre adolescentes e jovens, pelo simples fato de se pensar que se não há fumaça para ser tragada não ocorrerão problemas pulmonares (VIEGAS, 2008; RICKERT et al.; 2009).

O estudo da composição química inorgânica elementar, concentração de radionuclídeos naturais e a estimativa de dose gerada nos fumantes são realizados há muitos anos em todo o mundo, porém ainda existem poucos trabalhos na literatura sobre os derivados de tabaco produzidos, comercializados e consumidos no Brasil. 


\section{OBJETIVOS}

O presente trabalho teve como objetivos a determinação dos radionuclídeos naturais ${ }^{238} \mathrm{U},{ }^{226} \mathrm{Ra},{ }^{210} \mathrm{~Pb}$ e ${ }^{210} \mathrm{Po}$ da série de decaimento do ${ }^{238} \mathrm{U}$, ${ }^{232} \mathrm{Th}$ e ${ }^{228} \mathrm{Ra}$ da série de decaimento do ${ }^{232} \mathrm{Th}$ e a concentração de elementos maiores e traços, Al, As, Ba, Br, Ca, Cd, Ce, Co, Cr, Cs, Eu, Fe, Hf, Hg, I, K, La, Lu, $\mathrm{Mg}, \mathrm{Mn}, \mathrm{Nd}, \mathrm{P}, \mathrm{Rb}, \mathrm{S}, \mathrm{Sb}, \mathrm{Sc}, \mathrm{Se}, \mathrm{Sm}, \mathrm{Ta}, \mathrm{Tb}, \mathrm{Th}, \mathrm{U}, \mathrm{Yb}$ e Zn em derivados da Nicotiana tabacum L. produzidos e comercializados no Brasil e também a dose efetiva anual devido ao uso destes derivados. As técnicas analíticas utilizadas foram a análise por ativação com nêutrons instrumental - INAA e fluorescência de raios $X$ para a determinação de ${ }^{238} \mathrm{U},{ }^{232} \mathrm{Th}$, elementos maiores e traços, a medida alfa e beta total após separação radioquímica para a determinação dos radionuclídeos ${ }^{226} \mathrm{Ra},{ }^{210} \mathrm{~Pb}$ e ${ }^{228} \mathrm{Ra}$, e espectrometria alfa após separação radioquímica para o radionuclídeo ${ }^{210} \mathrm{Po}$. 


\section{PARTE EXPERIMENTAL}

\subsection{Amostragem}

Todas as amostras analisadas de produtos derivados da Nicotiana tabacum $L$. foram adquiridas em comércios populares e tabacarias nacionais

Os derivados de tabaco escolhidos para a amostragem foram: cigarros não aromatizados, cigarros aromatizados (mentolados e de cravo), charutos, cigarros de palha (folhas de tabaco secas e desfiadas, envoltas em palhas de milho) e fumo de corda (folhas de tabaco secas enroladas - formato de rolo e desfiado; natural e aromatizado). O fumo de corda pode ser consumido queimado em cigarros de palha ou papel, em cachimbos ou na forma não queimada inalando o pó diretamente ou mascado. Além destes foram também analisados amostras de rapé que são folhas de tabaco torradas e moídas, em pó, podendo ser acrescido de outras substâncias como aromatizantes - forma de uso inalado. Na FIG. 3.1 são apresentados exemplos de derivados de tabaco: cigarros, cigarros de palha, charutos, fumo de corda em rolo e rapé, respectivamente

No total foram analisadas 82 amostras sendo 20 de cigarros convencionais, 9 de cigarros aromatizados, 14 de cigarros de palha, 6 charutos, 2 rapés e 31 de fumo de corda desfiado e em rolo.

Este trabalho buscou analisar uma grande variedade de derivados de tabaco para uma real caracterização dos principais produtos nacionais. Dentre os cigarros adquiridos foram analisados os produtos das três principais empresas do seguimento no mercado brasileiro, as principais e mais consumidas marcas, variações de preço e de intensidades. 
FIGURA 3.1 - Exemplos de derivados de tabaco (a- cigarro; b- cigarro de palha; c - charuto; d - fumo de corda; e - rapé)

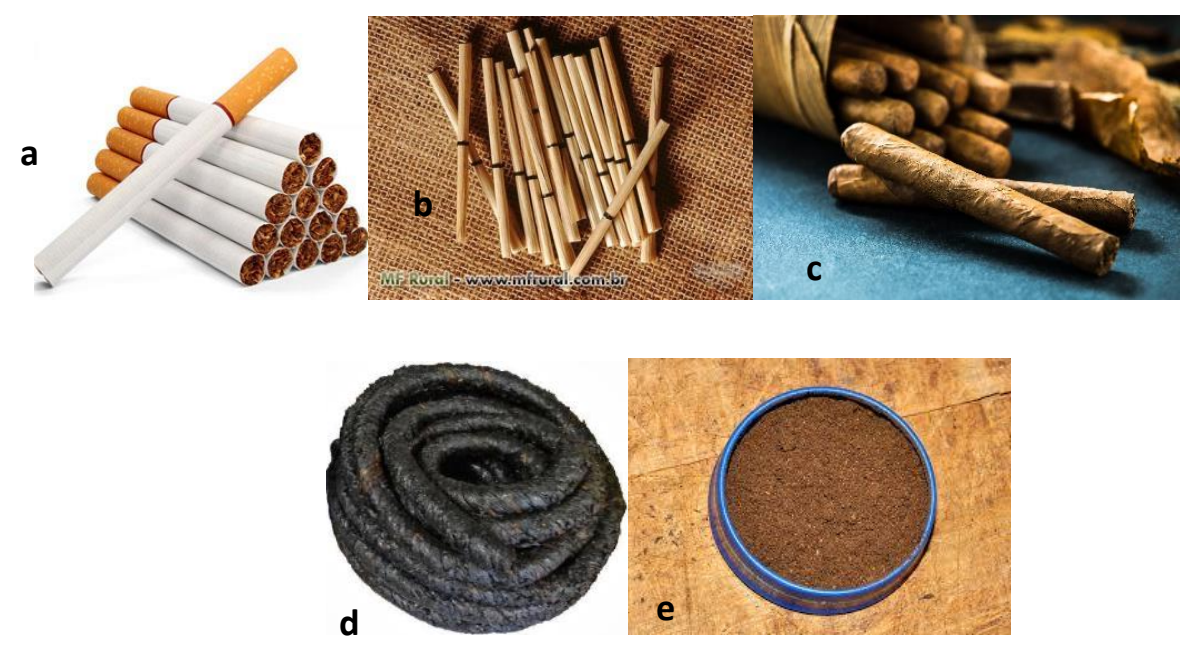

\subsection{Metodologias Analíticas}

Foram empregadas as técnicas analíticas: Análise por Ativação com Nêutrons Instrumental - INAA, Espectrometria de Fluorescência de Raios-X, para determinar dos elementos maiores, traços e terras raras; medida alfa e beta total e espectrometria alfa após separação radioquímica, para a determinação de ${ }^{226} \mathrm{Ra}$, ${ }^{210} \mathrm{~Pb}$ e ${ }^{210} \mathrm{Po}$ da série de decaimento natural do ${ }^{238} \mathrm{U}$ e ${ }^{228} \mathrm{Ra}$ da série de decaimento natural do ${ }^{232} \mathrm{Th}$.

Para as técnicas analíticas INAA e EDXRF as amostras "in natura" foram retiradas de suas embalagens originais, removidos os papeis, palhas de milho e filtros, ficando exclusivamente a parte que continha o tabaco. Foram levadas a estufa a $50^{\circ} \mathrm{C}$ por aproximadamente $62 \mathrm{~h}$ e, posteriormente, maceradas e homogeneizadas em almofariz de vidro até ficarem na forma de pó, FIG. 3.2 amostras secas e maceradas. 
FIGURA 3.2 - amostras secas e maceradas.

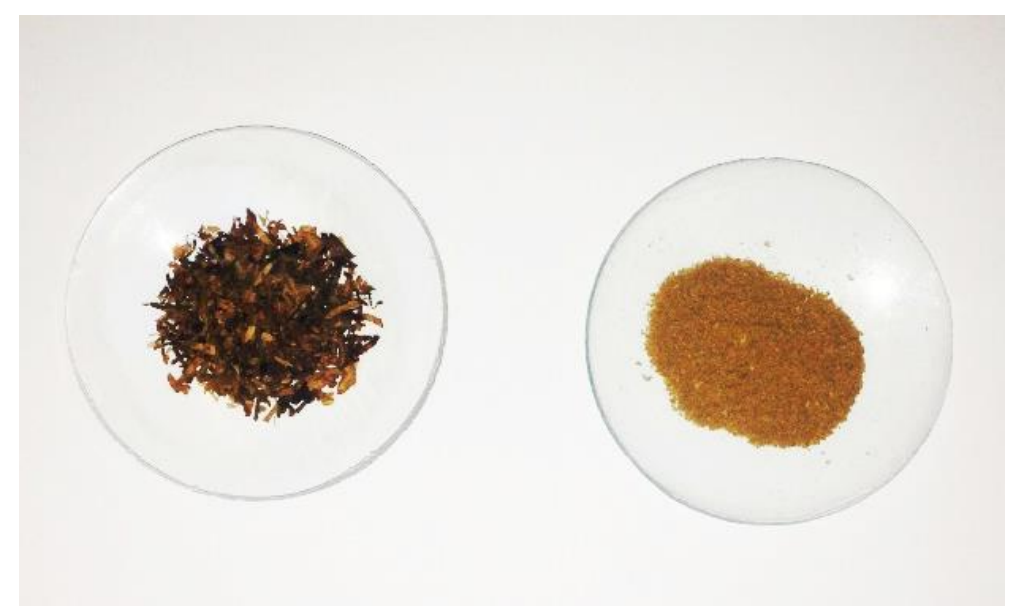

\subsubsection{Análise por Ativação com Nêutrons Instrumental (INAA)}

A INAA é uma técnica analítica não destrutiva que permite determinar até 40 elementos químicos a partir do bombardeamento por nêutrons em um reator nuclear, formando radionuclídeos artificiais em um estado altamente excitado, estes por sua vez sofrem desintegração nuclear emitindo raios gamas característicos (BODE, 1996).

Consiste em uma técnica de análise multielementar utilizada em diferentes áreas e para diferentes materiais como produtos agrícolas, produtos ambientais, produtos industriais, arqueologia, entre outros (SUSSA, 2017).

Neste trabalho a análise por ativação com nêutrons foi utilizada para a determinação de 27 elementos, sendo eles: $\mathrm{As}, \mathrm{Ba}, \mathrm{Br}, \mathrm{Ca}, \mathrm{Ce}, \mathrm{Co}, \mathrm{Cr}, \mathrm{Cs}, \mathrm{Eu}, \mathrm{Fe}$, $\mathrm{Hf}, \mathrm{K}, \mathrm{La}, \mathrm{Lu}, \mathrm{Na}, \mathrm{Nd}, \mathrm{Rb}, \mathrm{Sb}, \mathrm{Sc}, \mathrm{Se}, \mathrm{Sm}, \mathrm{Ta}, \mathrm{Tb}, \mathrm{Th}, \mathrm{U}, \mathrm{Yb}$ e Zn.

Aproximadamente de $250 \mathrm{mg}$ de amostra foram pesados e acondicionadas em sacos de polietileno previamente limpos, FIG. 3.3. As amostras foram irradiados por um período de 6h, sob um fluxo de nêutrons térmicos de $10^{12} \mathrm{n} \mathrm{cm}^{-2} \mathrm{~s}^{-1}$ no Reator de Pesquisa IEA-R1 do IPEN (DAMATTO, 2010).

Junto com as amostras foram irradiados materiais de referência certificado: Soil 1 da Agência Internacional de Energia Atômica - IAEA, Montanna II Soil e Tomato Leaves ambos do Instituto Nacional de Padrões e Tecnologia NIST (TAB. 3.1), as massas irradiadas dos materiais de referência foram aproximadamente $190 \mathrm{mg}$ de cada um. 
FIGURA 3.3 - Amostras acondicionadas para irradiação.

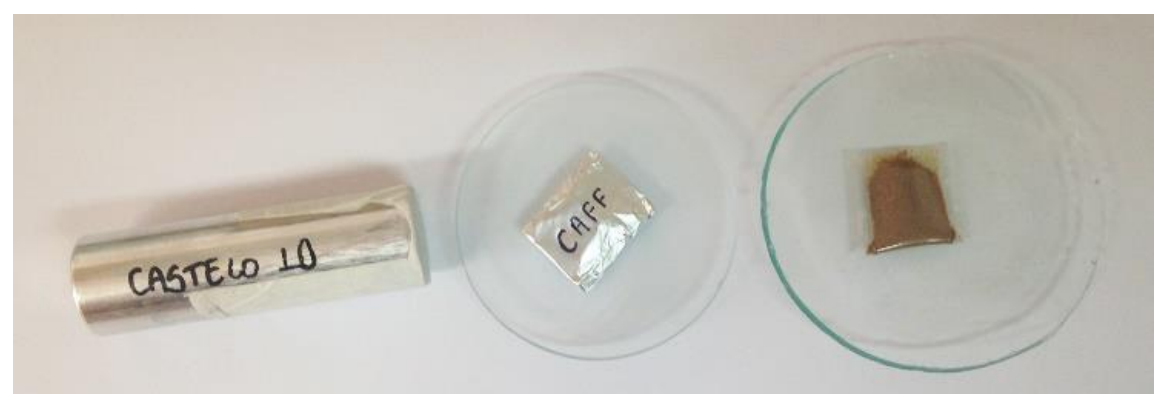

Os elementos $\mathrm{As}, \mathrm{Br}, \mathrm{K}, \mathrm{La}, \mathrm{Na}, \mathrm{Nd}, \mathrm{Sb}, \mathrm{Sm}$ e $\mathrm{U}$ foram determinados após 7 dias da irradiação das amostras e os demais elementos após 15 dias. Utilizou-se um detector germânio hiperpuro (HPGe) com janela de berílio modelo da marca ORTEC, com sistema eletrônico associado, Multicanal 919E ETHERNIM da ORTEC, um computador com programa emulador de multicanal Maestro da ORTEC (Maestro, 2001) e programa de análise de espectros InterWinnerWinnerGamma 6.0 da ORTEC (ORTEC. INTERWINNERTM 6.0 MCA, 2004 para a medida dos emissores gama, e a FIG. 3.5 mostra um exemplo de espectro obtido na análise multielementar por INAA.

FIGURA 3.4 - Detectores de germânio hiperpuro (HPGe).

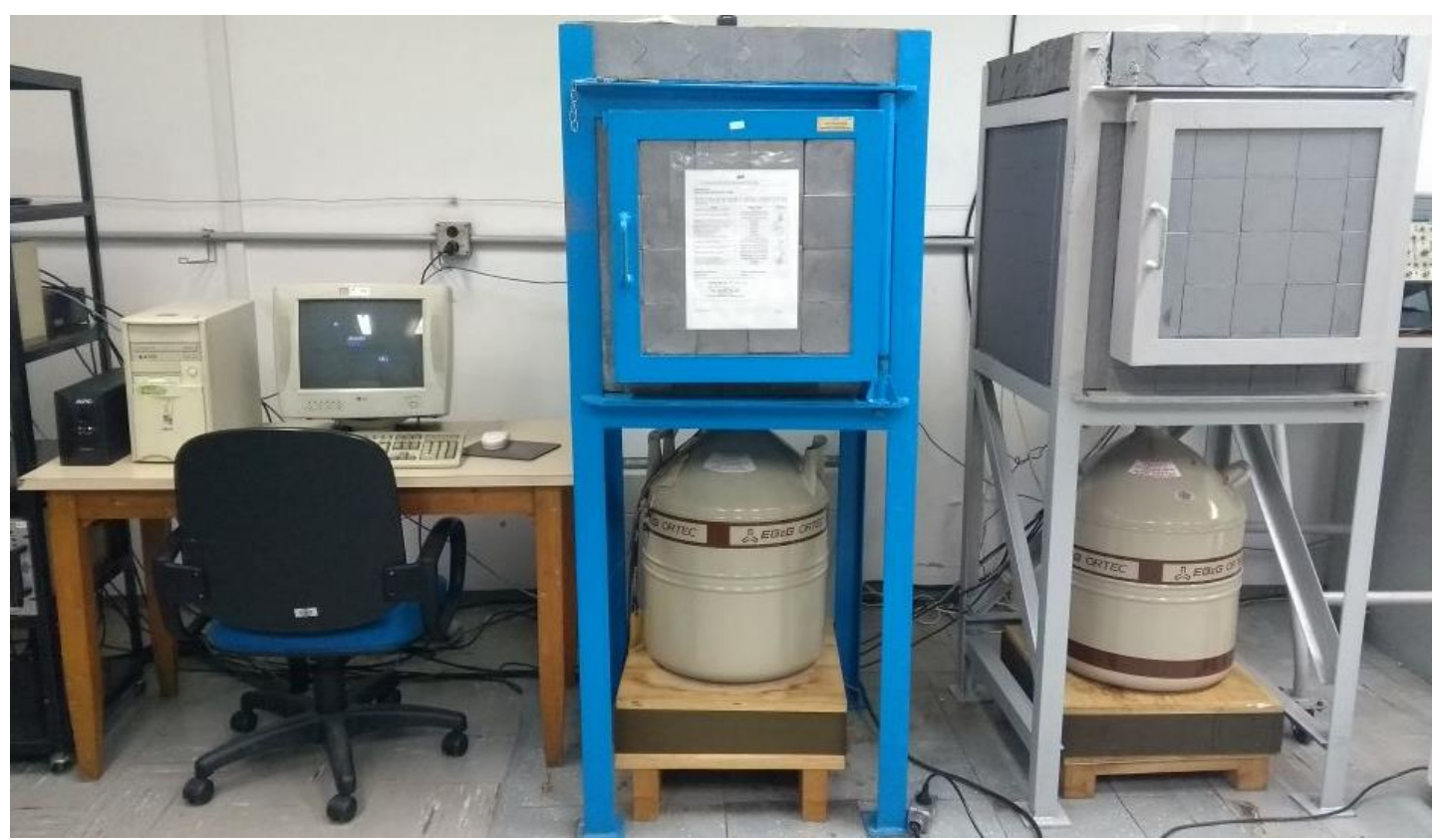


FIGURA 3.5 - Espectro obtido na análise multielementar por INAA

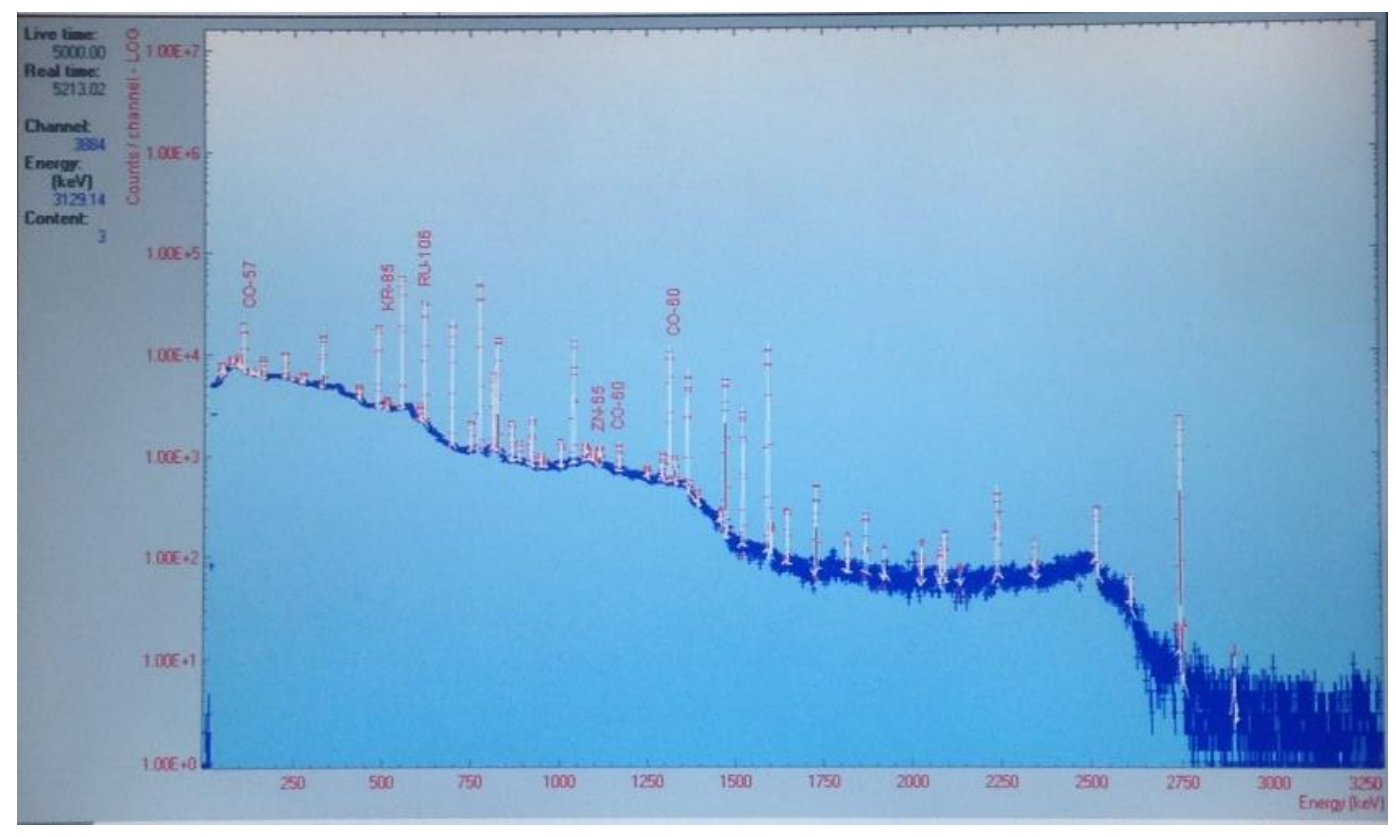

Os radioisótopos formados pela ativação foram identificados pelas suas energias de raios gama e as concentrações foram calculadas pela comparação de áreas de picos da amostra e dos padrões que foram ativados simultaneamente. Após a irradiação ambos são medidos em condição geométrica idêntica pelo detector. Para o cálculo foi utilizada a expressão (3.1) (BODE,1996).

$$
C_{a i}=\left(A^{i} \cdot m p \cdot C_{p i}\right) \cdot e^{\lambda \cdot(t a-t p)} / A_{p}{ }^{i} \cdot m a
$$

Em que:

$\mathrm{Ca}^{\mathrm{i}}$ : Concentração do elemento i na amostra em $\mathrm{mg} \mathrm{kg}^{-1}$;

$\mathrm{Cp}^{\mathrm{i}}$ : Concentração do elemento i no padrão em $\mathrm{mg} \mathrm{kg}^{-1}$;

$A_{a}{ }^{i}$ : Atividade do elemento i na amostra em cps;

$A_{p}^{i}$ : Atividade do elemento i no padrão em cps;

$m_{a}$ e $m_{p}$ : massas da amostra e padrão, respectivamente em $\mathrm{g}$;

$\lambda$ constante de decaimento do radioisótopo em min $^{-1}$;

$t_{a}$ - $t_{p}$ : diferença de tempo entre as contagens da amostra e padrão em minutos

Na TAB 3.1 são apresentados os elementos determinados na INAA com suas respectivas concentrações nos materiais de referência em $\mathrm{mg} \mathrm{kg}^{-1}$, os radioisótopos formados na INAA com suas energias de raios gama utilizados na espectrometria gama e suas meias-vidas. 
TABELA 3.1 - Elementos determinados por análise por ativação neutrônica, concentrações dos elementos nos materiais de referência $\left(\mathrm{mg} \mathrm{kg}^{-1}\right)$, radioisótopos formados na ativação, energia dos raios gama utilizados na espectrometria gama e meias-vidas.

\begin{tabular}{|c|c|c|c|c|c|c|}
\hline Elemento & Soil 1 & $\begin{array}{c}\text { Montanna II } \\
\text { Soil }\end{array}$ & $\begin{array}{l}\text { Tomato } \\
\text { Leaves }\end{array}$ & Radioisótopo & $E(\mathrm{KeV})$ & $\begin{array}{l}\text { Meia } \\
\text { Vida }\end{array}$ \\
\hline As & $27,6 \pm 2,9$ & $107 \pm 5$ & $0,112 \pm 0,004$ & ${ }^{76}$ As & 559,1 & $26,32 \mathrm{~h}$ \\
\hline $\mathrm{Ba}$ & $639 \pm 53$ & $730 \pm 15$ & 63 & ${ }^{131} \mathrm{Ba}$ & 496,33 & $11,8 d$ \\
\hline $\mathrm{Br}$ & & & 1300 & ${ }^{82} \mathrm{Br}$ & 554 e 776 & $35,5 h$ \\
\hline $\mathrm{Ca}$ & & $24200 \pm 600$ & $50500 \pm 900$ & ${ }^{47} \mathrm{Ca}$ & 1296,09 & $4,54 d$ \\
\hline $\mathrm{Ce}$ & $117 \pm 17$ & $6,7 \pm 0,2$ & 2 & ${ }^{141} \mathrm{Ce}$ & 145,44 & $32,5 d$ \\
\hline Co & $19,8 \pm 1,5$ & $9,89 \pm 0,18$ & $0,57 \pm 0,02$ & ${ }^{60} \mathrm{Co}$ & $\begin{array}{c}1173,24 \mathrm{e} \\
1332,5\end{array}$ & $5,72 a$ \\
\hline $\mathrm{Cr}$ & $104 \pm 9$ & $52,3 \pm 2,9$ & $1,99 \pm 0,006$ & ${ }^{57} \mathrm{Cr}$ & 320,08 & $27,7 d$ \\
\hline Cs & $7 \pm 0,9$ & & 0,053 & ${ }^{134} \mathrm{Cs}$ & 795,85 & $2,06 a$ \\
\hline Eu & $1,6 \pm 0,5$ & $1,1 \pm 0,2$ & & ${ }^{152} \mathrm{Eu}$ & $\begin{array}{l}121,78 \mathrm{e} \\
1408,01\end{array}$ & $13,33 a$ \\
\hline $\mathrm{Fe}$ & $\begin{array}{c}67400 \pm \\
1700\end{array}$ & $28200 \pm 400$ & $368 \pm 7$ & ${ }^{59} \mathrm{Fe}$ & $\begin{array}{c}1099,25 \text { e } \\
1291,6\end{array}$ & $44,5 d$ \\
\hline $\mathrm{Hf}$ & $4,2 \pm 0,6$ & $9,2 \pm 0,2$ & 0,14 & ${ }^{181} \mathrm{Hf}$ & 482,18 & $742,39 d$ \\
\hline $\mathbf{K}$ & $\begin{array}{c}14500 \pm \\
2100\end{array}$ & $25300 \pm 1000$ & $27000 \pm 500$ & ${ }^{42} \mathrm{~K}$ & 1524,7 & $12,36 \mathrm{~h}$ \\
\hline La & $52,6 \pm 3,1$ & $38 \pm 1$ & 2,3 & ${ }^{140} \mathrm{La}$ & $\begin{array}{c}328,76 \text { e } \\
1596,21\end{array}$ & $44,5 d$ \\
\hline Lu & $0,54 \pm 0,13$ & & & ${ }^{177} \mathrm{Lu}$ & 208,37 & $6,71 d$ \\
\hline $\mathrm{Na}$ & $1700 \pm 100$ & $12000 \pm 100$ & $136 \pm 4$ & ${ }^{24} \mathrm{Na}$ & 1368,68 & $14,95 \mathrm{~h}$ \\
\hline Nd & & $29 \pm 2$ & & ${ }^{147} \mathrm{Nd}$ & 91,1 e 531,02 & $10,98 d$ \\
\hline $\mathbf{R b}$ & $113 \pm 11$ & $120 \pm 3$ & $14,89 \pm 0,27$ & ${ }^{86} \mathrm{Rb}$ & 1076,64 & $18,66 \mathrm{~d}$ \\
\hline $\mathrm{Sb}$ & $1,31 \pm 0,12$ & $23,8 \pm 1,4$ & $0,063 \pm 0,006$ & ${ }^{122} \mathrm{Sb}$ & 564,12 & $2,7 d$ \\
\hline Sc & $17,3 \pm 1,1$ & $8,5 \pm 0,1$ & 0,1 & ${ }^{46} \mathrm{Sc}$ & 889,28 & $83,81 d$ \\
\hline $\mathrm{Se}$ & $2,85 \pm 0,14$ & 2 & $0,054 \pm 0,003$ & & & \\
\hline Sm & $9,25 \pm 0,51$ & $5,93 \pm 0,28$ & 0,19 & ${ }^{153} \mathrm{Sm}$ & 103,18 & $46,27 \mathrm{~h}$ \\
\hline $\mathrm{Ta}$ & $1,58 \pm 0,58$ & 1 & & ${ }^{182} \mathrm{Ta}$ & $\begin{array}{c}1189,05 \text { e } \\
1221,41\end{array}$ & $114,5 d$ \\
\hline $\mathrm{Tb}$ & $1,4 \pm 0,46$ & 0,8 & & ${ }^{160} \mathrm{~Tb}$ & 879,38 & $72,3 d$ \\
\hline Th & $14 \pm 1$ & $15 \pm 1$ & 0,12 & ${ }^{233} \mathrm{Th}$ & 312,01 & $27 d$ \\
\hline $\mathbf{U}$ & $4,02 \pm 0,33$ & $3,01 \pm 0,12$ & 0,035 & ${ }^{239} \mathrm{~Np}$ & 228,18 e 277,6 & $2,36 d$ \\
\hline $\mathrm{Yb}$ & $3,42 \pm 0,65$ & 3 & & ${ }^{169} \mathrm{Yb}$ & $\begin{array}{c}177,21 \mathrm{e} \\
197,98\end{array}$ & $32,02 d$ \\
\hline $\mathrm{Zn}$ & $223 \pm 20$ & $414 \pm 11$ & $30,9 \pm 0,7$ & ${ }^{65} \mathrm{Zn}$ & 1115,6 & $243,9 d$ \\
\hline
\end{tabular}

Os valores apresentados sem os respectivos desvios padrões correspondem a valores informativos 


\subsubsection{Espectrometria de Fluorescência de Raios-X (EDXRF)}

A técnica de EDXRF foi usada para determinação das concentrações de elementos químicos $\mathrm{Al}, \mathrm{As}, \mathrm{Ba}, \mathrm{Br}, \mathrm{Ca}, \mathrm{Cd}, \mathrm{Ce}, \mathrm{Cl}, \mathrm{Co}, \mathrm{Cr}, \mathrm{Cs}, \mathrm{Cu}, \mathrm{Fe}, \mathrm{Hg}, \mathrm{K}$, La, $\mathrm{Mg}, \mathrm{Mn}, \mathrm{Ni}, \mathrm{Nd}, \mathrm{P}, \mathrm{Pb}, \mathrm{Rb}, \mathrm{S}, \mathrm{Sb}, \mathrm{Se}, \mathrm{Sm}, \mathrm{Sr}, \mathrm{Th}, \mathrm{U}, \mathrm{Zn}$, sendo uma técnica não destrutiva e sem necessidade de pré-tratamentos. As amostras foram colocadas em um suporte para amostra com filme de polipropileno.

O equipamento utilizado foi o EDX-720 da Shimadzu Corporation do Centro de Química e Meio Ambiente do IPEN/CNEN-SP, e os parâmetros de operação foram: tubo de raios $X$ de Ródio, tensão de $\mathrm{Na}$ a Sc de $15 \mathrm{kV}$ e de Ti a $\mathrm{V}$ de $50 \mathrm{kV}$; corrente ajustada automaticamente de no máximo $1 \mathrm{~mA}$; colimador de 5 $\mathrm{mm}$; detector de Si-Li; resfriamento com $\mathrm{N}_{2}$ liquido; tempo de medida de 100 s e 6 repetições por amostra (BONA et all, 2007).

Para calcular a curva de calibração e verificar a precisão e exatidão da metodologia foram utilizados três materiais de referência: Tomato Leaves e Peach Leaves ambos do Instituto Nacional de Padrões e Tecnologia - NIST e o Sargasso (CRM no9) do Instituto Nacional para Estudos Ambientais - NIES.

Na TAB3.2 são apresentados os elementos determinados pelo método EDXRF com suas respectivas concentrações nos materiais de referência ( $\mathrm{mg} \mathrm{kg}^{-1}$ $\mathrm{e} * \%)$. 
TABELA 3.2 - Concentrações nos materiais de referência (mg kg $\left.{ }^{-1},{ }^{*} \%\right)$, usados na calibração e validação dos resultados pela análise por EDXRF.

\begin{tabular}{|c|c|c|c|}
\hline Elemento & Peach Leaves & Sargasso & Tomato Leaves \\
\hline Al & $249 \pm 8$ & 215 & $598 \pm 12$ \\
\hline As & $115 \pm 9$ & $107 \pm 5$ & $0,112 \pm 0,004$ \\
\hline $\mathbf{B a}$ & $124 \pm 4$ & & 63 \\
\hline $\mathrm{Br}$ & 11 & 270 & 1300 \\
\hline${ }^{\star} \mathrm{Ca}$ & $1,56 \pm 0,02$ & $1,34 \pm 0,05$ & $5,05 \pm 0,09$ \\
\hline Cd & 0,03 & $0,15 \pm 0,02$ & $1,52 \pm 0,04$ \\
\hline $\mathrm{Ce}$ & 10 & & 2 \\
\hline $\mathrm{Cl}$ & $360 \pm 19$ & 51.000 & 6600 \\
\hline Co & 0,07 & $0,12 \pm 0,01$ & $0,57 \pm 0,02$ \\
\hline $\mathrm{Cr}$ & 1 & 0,2 & $1,99 \pm 0,006$ \\
\hline Cs & & 0,04 & 0,053 \\
\hline $\mathrm{Cu}$ & $3,7 \pm 0,4$ & $4,9 \pm 0,2$ & $4,70 \pm 0,14$ \\
\hline $\mathbf{F e}$ & 220 & $187 \pm 6$ & $368 \pm 7$ \\
\hline $\mathrm{Hg}$ & $0,031 \pm 0,007$ & 0,04 & $0,034 \pm 0,004$ \\
\hline I & 0,3 & 520 & 0,85 \\
\hline${ }^{\star} \mathrm{K}$ & $2,43 \pm 0,03$ & $6,10 \pm 0,20$ & $2,7 \pm 0,05$ \\
\hline La & 9 & & 2,3 \\
\hline${ }^{\star} \mathbf{M g}$ & $0,432 \pm 0,008$ & $0,65 \pm 0,03$ & 1,2 \\
\hline Mn & $98 \pm 3$ & $21,2 \pm 1,0$ & $246 \pm 8$ \\
\hline $\mathrm{Na}$ & $24 \pm 2$ & $1,70 \pm 0,08$ & $136 \pm 4$ \\
\hline Nd & 7 & & \\
\hline $\mathbf{N i}$ & $0,69 \pm 0,09$ & & $1,59 \pm 0,07$ \\
\hline${ }^{\star} \mathbf{P}$ & & 0,26 & $0,216 \pm 0,004$ \\
\hline $\mathbf{P b}$ & $0,87 \pm 0,03$ & $1,35 \pm 0,05$ & \\
\hline $\mathbf{R b}$ & 19 & $24 \pm 2$ & $14,89 \pm 0,27$ \\
\hline${ }^{\star} S$ & 0,2 & 1,2 & 0,96 \\
\hline $\mathrm{Sb}$ & 0,02 & 0,04 & $0,063 \pm 0,006$ \\
\hline $\mathrm{Se}$ & $0,120 \pm 0,009$ & 0,05 & $0,054 \pm 0,003$ \\
\hline Sm & 1 & & 0,19 \\
\hline $\mathrm{Sr}$ & $53 \pm 4$ & $0,100 \pm 0,003$ & 85 \\
\hline Th & 0,05 & & 0,12 \\
\hline $\mathbf{U}$ & 0,015 & 0,4 & 0,035 \\
\hline $\mathbf{V}$ & $0,37 \pm 0,03$ & $1,0 \pm 0,1$ & $0,835 \pm 0,010$ \\
\hline $\mathrm{Zn}$ & $17,9 \pm 0,4$ & $15,6 \pm 1,2$ & $30,9 \pm 0,7$ \\
\hline
\end{tabular}

Os valores apresentados sem os respectivos desvios padrões correspondem a valores informativos 


\subsubsection{Metodologia de determinação ${ }^{226} \mathrm{Ra},{ }^{228} \mathrm{Ra}$ e ${ }^{210} \mathrm{~Pb}$}

Na determinação dos radionuclídeos ${ }^{226} \mathrm{Ra},{ }^{228} \mathrm{Ra}$ e ${ }^{210} \mathrm{~Pb}$ foi utilizada a metodologia de medida alfa e beta total após separação radioquímica por apresentar como característica principal uma baixa radiação de fundo e consequentemente um limite inferior de detecção também baixo, ideal para quantificar radionuclídeos em amostras ambientais (OLIVEIRA, 1993)

A análise sequencial dos radionuclídeos é composta por pré-tratamento da amostra, separação radioquímica dos radionuclídeos por coprecipitações, determinação dos rendimentos químicos do método e medida alfa e beta total. $A$ técnica também é vantajosa por eliminar cátions interferente de elementos químicos estáveis como o $\mathrm{Al}^{3+}$, o $\mathrm{Fe}^{3+}$, o $\mathrm{Ca}^{2+}$ entre outros e outros radionuclídeos naturais (OLIVEIRA, 1993).

\subsubsection{Determinação de ${ }^{226} \mathrm{Ra}$ e ${ }^{228} \mathrm{Ra}$}

As amostras foram retiradas de sua embalagem, removidos o papel envolvente e filtro, em seguida pesadas em balança analítica, aproximadamente $2,5 \mathrm{~g}$ de cada amostra, em béqueres codificados (FIG. 3.6), adicionados aproximadamente $50 \mathrm{~mL}$ de $\mathrm{HNO}_{3}$ conc. (PA), $1 \mathrm{~mL}$ de carregador de $\mathrm{Ba}^{2+}$ (20 mg Ba mL-1) e $1 \mathrm{~mL}$ de carregador de $\mathrm{Pb}^{2+}\left(20 \mathrm{mg} \mathrm{Pb} \mathrm{mL}^{-1}\right.$ ) e deixadas em repouso por aproximadamente 48 horas para digestão a frio. Após este período as amostras foram levadas à chapa aquecedora em temperatura de aproximadamente $200^{\circ} \mathrm{C}$, para dissolução e adicionou-se $\mathrm{H}_{2} \mathrm{O}_{2} 40 \%$ para eliminação da matéria orgânica (FIG. 3.7).

FIGURA 3.6 - Amostras de derivados de tabaco pesadas

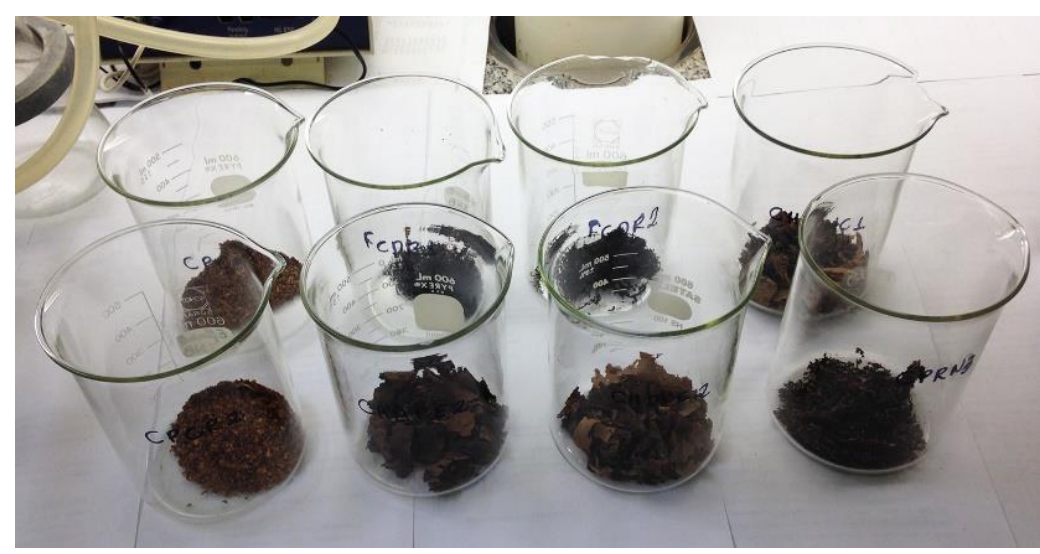


FIGURA 3.7 - Amostras em processo de dissolução (a) e eliminação da matéria orgânica (b)

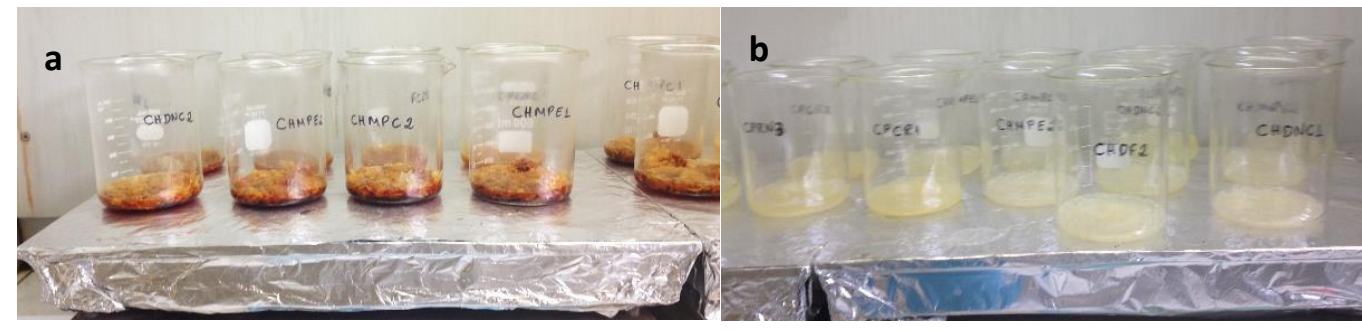

As amostras foram transferidas para béqueres de $2 \mathrm{~L}$ e o volume elevado a 1 L. Adicionou-se $70 \mathrm{~mL}$ de ácido sulfúrico $3 \mathrm{~mol} \mathrm{~L}^{-1}$ sob agitação para a precipitação; aguardou-se até o dia seguinte para decantar todo o precipitado. O sobrenadante foi descartado com uso de uma bomba à vácuo e o precipitado foi transferido para tubo de centrifuga com ajuda água superpura para lavagem; centrifugado a $2000 \mathrm{rpm}$ por $10 \mathrm{~min}$. O sobrenadante resultante foi descartado e o precipitado foi lavado com ácido sulfúrico 0,1 $\mathrm{mol} \mathrm{L}^{-1}$. Novamente a amostra foi centrifugada e descartado o sobrenadante.

Ao precipitado foram adicionados $2,0 \mathrm{~g}$ de ácido nitrilotriacético - NTA (Titriplex I), $40 \mathrm{~mL}$ de água superpura, indicador vermelho de metila e $7 \mathrm{~mL}$ de $\mathrm{NaOH} 6 \mathrm{~mol} \mathrm{~L}^{-1}$, em seguida foi aquecido em banho-maria até a solubilização total do precipitado. Adicionou-se $5 \mathrm{~mL}$ de sulfato de amônio $\left(25 \mathrm{~g} \mathrm{~L}^{-1}\right)$ e ácido acético glacial para ajuste do $\mathrm{pH}$ entre 4,5 - 5,0, precipitando assim o sulfato de bário e rádio e mantendo o chumbo complexado em solução. Deixou-se a solução decantar até o dia seguinte.

No dia seguinte a solução foi centrifugada a 2000 rpm por 10 min e o sobrenadante foi separado para análise de chumbo. O precipitado foi lavado com acetato de amônio 20\% e centrifugado novamente, sendo o sobrenadante resultante adicionado ao anterior.

Adicionou ao precipitado de sulfato de bário e rádio 2,0 g de ácido diaminotetraacético sal dissódico (EDTA), $40 \mathrm{~mL}$ de água e $7 \mathrm{~mL}$ de hidróxido de amônio 25\%. Em seguida a amostra foi levada à chapa aquecedora em banhomaria até a solubilização total do precipitado. Adicionou-se $5 \mathrm{~mL}$ de sulfato de amônio (25 $\left.\mathrm{g} \mathrm{L}^{-1}\right)$ e ácido acético glacial precipitando novamente o sulfato de bário e rádio final. No dia seguinte a solução foi filtrada com filtro de fibra de vidro, FIG. 3.8. 
FIGURA 3.8 - Precipitado de $\mathrm{Ba}(\mathrm{Ra}) \mathrm{SO}_{4}$

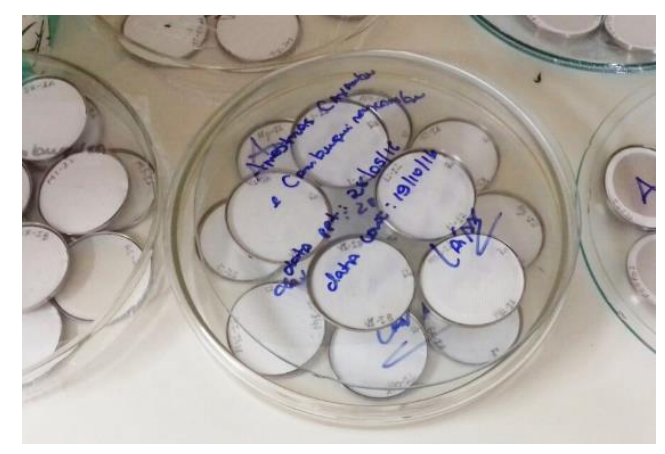

O ${ }^{226} \mathrm{Ra}$ e o ${ }^{228} \mathrm{Ra}$ foram determinado após 21 dias da precipitação usando a medida alfa e beta total do precipitado de sulfato de bário e rádio respectivamente em um detector proporcional de fluxo gasoso de baixa radiação de fundo.

A concentração de atividade do ${ }^{226} \mathrm{Ra}$ foi determinada pela expressão (3.2):

$$
\mathrm{A}\left({ }^{226} R a\right)=\frac{R_{a}-R_{0}}{\mathrm{Rq} \cdot \mathrm{E} \cdot \mathrm{m} \cdot \mathrm{fabs} \cdot\left[1+\mathrm{k}\left(1-\mathrm{e}^{-\lambda \mathrm{t}}\right)\right]}
$$

Em que:

$A\left({ }^{226} \mathrm{Ra}\right)=$ concentração de atividade de ${ }^{226} \mathrm{Ra}$ na amostra em mBq g-1

$\mathrm{R}_{\mathrm{a}}=$ taxa de contagem total da amostra em cps

$\mathrm{R}_{0}=$ taxa de contagem da radiação de fundo em cps

$\mathrm{Rq}=$ rendimento químico

$E=$ eficiência de contagem em $\mathrm{cpm} \mathrm{dps}^{-1}$

$\mathrm{m}=$ massa da amostra em $\mathrm{g}$

fabs $\left({ }^{226} \mathrm{Ra}\right)=$ coeficiente de auto absorção do ${ }^{226} \mathrm{Ra}$ no precipitado de $\mathrm{Ba}(\mathrm{Ra}) \mathrm{SO}_{4}$

$\lambda=$ constante de desintegração do ${ }^{222} \operatorname{Rn}\left(0,181 d^{-1}\right)$

$\mathrm{t}=$ tempo transcorrido entre a precipitação do $\mathrm{Ba}(\mathrm{Ra}) \mathrm{SO}_{4}$ e a contagem em dias $\mathrm{k}=$ constante de auto absorção

O rendimento químico $(\mathrm{Rq})$ do procedimento foi determinado gravimetricamente a partir da massa de carregador de bário adicionado; $1 \mathrm{~mL}$ de carregador de bário $\left(20 \mathrm{mg} \mathrm{mL}^{-1}\right)$ correspondente à 34,0 mg Ba(Ra)SO4. 
A concentração de atividade de ${ }^{228} \mathrm{Ra}$ foi determinada segundo a expressão (3.3):

$$
\mathrm{A}\left({ }^{228} R a\right)=\frac{\mathrm{Rn}-\mathrm{Bg}}{60 \cdot \mathrm{Rq} \cdot \operatorname{Ef}\left({ }^{228} R a\right) \cdot \mathrm{m}}-\frac{\operatorname{Ef}\left({ }^{226} R a\right) \cdot \mathrm{A}\left(2^{226} R a\right)}{\operatorname{Ef\beta }\left({ }^{228} R a\right)}
$$

Em que:

$\mathrm{A}\left({ }^{228} \mathrm{Ra}\right)=$ concentração de atividade do ${ }^{228} \mathrm{Ra}$, em $\mathrm{mBq} \cdot \mathrm{g}^{-1}$;

$\mathrm{Rn}=$ taxa de contagem beta total obtida em cps;

$\mathrm{Bg}=$ taxa de radiação de fundo beta total em cps;

$\mathrm{RQ}=$ rendimento químico;

$\mathrm{EF} \beta\left({ }^{228} \mathrm{Ra}\right)$ = eficiência de contagem beta total, calculada pela medida de um precipitado padrão de $\mathrm{Ba}\left({ }^{228} \mathrm{Ra}\right) \mathrm{SO}_{4}$ em dps ${ }^{-1}$;

$\mathrm{EF} \beta\left({ }^{226} \mathrm{Ra}\right)$ = eficiência de contagem beta total, calculada pela medida de um precipitado padrão de $\mathrm{Ba}\left({ }^{226} \mathrm{Ra}\right) \mathrm{SO} 4$ em dps ${ }^{-1}$;

$\mathrm{A}\left({ }^{226} \mathrm{Ra}\right)=$ concentração de atividade calculada para o ${ }^{226} \mathrm{Ra}$, em Bq g ${ }^{-1}$;

$\mathrm{m}=$ massa da amostra em $\mathrm{g}$

\subsubsection{Determinação do ${ }^{210} \mathrm{~Pb}$ (MOREIRA, 1993)}

A solução de chumbo complexado foi aquecida em banho-maria e

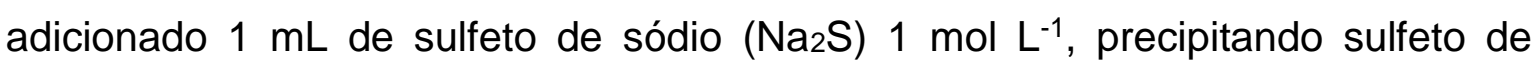
chumbo.

Após a decantação do precipitado, a suspenso foi centrifugada a 2000 rpm por 30 min, o sobrenadante foi descartado, o precipitado lavado com água superpura. O processo de centrifugação foi repetido e descartado o sobrenadante. O precipitado foi dissolvido com ácido nítrico concentrado a quente.

A solução foi filtrada com papel qualitativo com auxílio de água superpura e adicionou-se ao filtrado acetato de amônio 40\% até ajuste do pH entre 4,5 - 5,0. Aqueceu-se a solução até ebulição e adicionou-se 2,5 mL de cromato de sódio 30\%, precipitando o chumbo na forma de cromato. No dia seguinte após decantação, a mistura foi filtrada a vácuo com filtro de fibra de vidro, FIG. 3.9. 
FIGURA 3.9 - Precipitado de $\mathrm{PbCrO}_{4}$

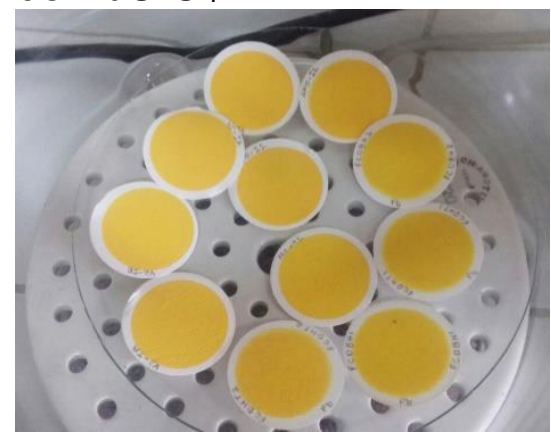

O rendimento químico do procedimento também foi determinado gravimetricamente a partir da massa de carregador de chumbo adicionado; $1 \mathrm{~mL}$ de carregador de $\mathrm{Pb}\left(20 \mathrm{mg} \mathrm{Pb} \mathrm{mL}^{-1}\right.$ ) corresponde a uma massa de 31,2 $\mathrm{mg}$ $\mathrm{PbCrO}_{4}$. O ${ }^{210} \mathrm{~Pb}$ foi determinado após 10 dias da precipitação, pela medida beta total do precipitado $\mathrm{PbCrO}_{4}$, em um detector proporcional de fluxo gasoso de baixa radiação de fundo, FIG. 3.10.

FIGURA 3.10 - Detector proporcional de fluxo gasoso de baixa radiação de fundo.

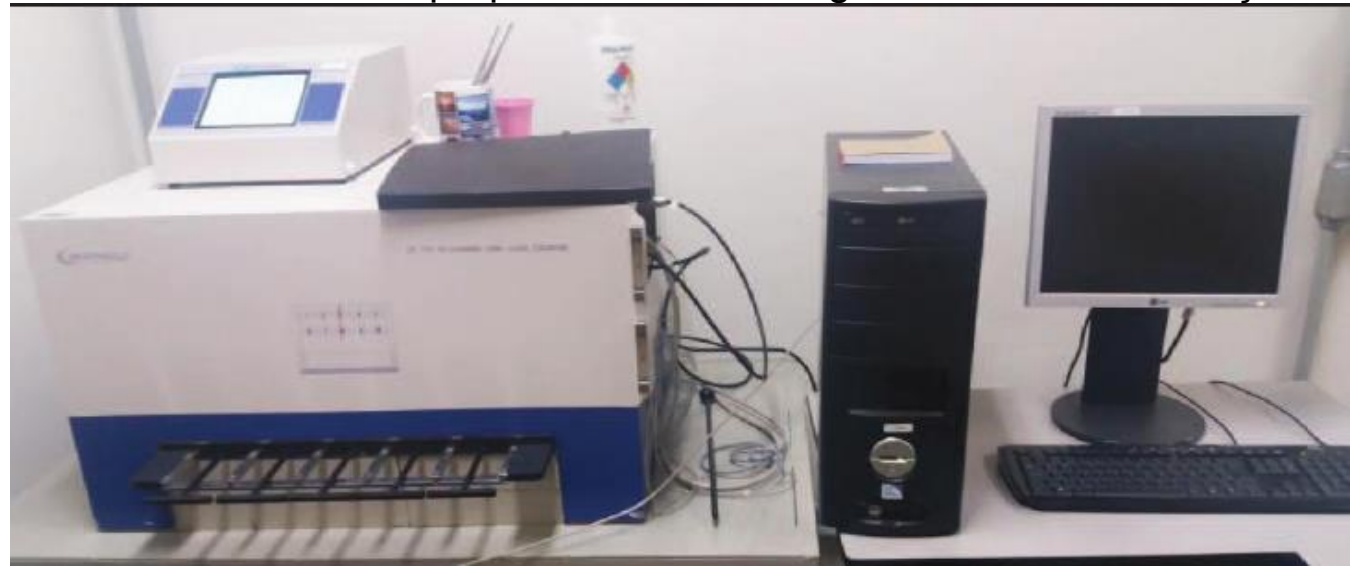

A concentração de atividade de ${ }^{210} \mathrm{~Pb}$ foi determinada segundo a expressão (3.4):

$$
\mathrm{A}\left({ }^{210} P b\right)=\frac{R_{a}-R_{0}}{\mathrm{Rq} \cdot \mathrm{E} \cdot \mathrm{m} \cdot\left(1-\mathrm{e}^{-\lambda \mathrm{t}}\right)}
$$

Em que:

$\mathrm{A}\left({ }^{210} \mathrm{~Pb}\right)=$ concentração de atividade de ${ }^{210} \mathrm{~Pb}$ na amostra em $\mathrm{mBq} \mathrm{g}^{-1}$

$\mathrm{R}_{\mathrm{a}}=$ taxa de contagem total da amostra em cpm

$\mathrm{R}_{0}=$ taxa de contagem da radiação de fundo em cpm

$\mathrm{Rq}=$ rendimento químico 
$E=$ eficiência de contagem em $\mathrm{cpm} \mathrm{dps}^{-1}$

$\mathrm{m}=$ massa da amostra em $\mathrm{g}$

$\mathrm{t}=$ tempo transcorrido entre a precipitação do $\mathrm{PbCrO}_{4} \mathrm{e}$ a contagem em dias $\lambda=$ constante de desintegração do ${ }^{210} \mathrm{Bi}\left(0,183 \mathrm{~d}^{-1}\right)$

\subsubsection{Determinação do ${ }^{210} \mathrm{Po}$}

A análise de ${ }^{210} \mathrm{Po}$ neste trabalho foi realizada por espectrometria alfa, após deposição espontânea em disco de cobre segundo Nieri Neto, (1996), que cita que uma importante propriedade do polônio é a sua deposição espontânea em diversas superfícies metálicas, como o cobre, níquel ou prata, a partir de soluções acidas, proporcionando a separação do polônio dos demais radionuclídeos naturais. Outra vantagem do método é a deposição homogênea de camada fina sendo possível a realização de espectrometria alfa após a deposição (MOREIRA, 1993; NIERI NETO, 1996).

O rendimento químico pode ser determinado adicionando ao método um traçador de polônio (NIERI NETO, 1996), que nesta metodologia foi usado o traçador ${ }^{209} \mathrm{Po}$.

As amostras foram retiradas de sua embalagem, removidos o papel envolvente e filtro, em seguida pesadas em balança analítica, aproximadamente $3,0 \mathrm{~g}$ de cada amostra, em béqueres previamente codificados, adicionados aproximadamente $50 \mathrm{~mL}$ de $\mathrm{HNO}_{3}$ conc. (PA), 0,1 mg de traçador ${ }^{209} \mathrm{Po}$, deixadas em repouso por aproximadamente 48 horas para digestão a frio. Após este período as amostras foram levadas à chapa aquecedora em temperatura de aproximadamente $70^{\circ} \mathrm{C}$, para dissolução e adicionou-se $\mathrm{H}_{2} \mathrm{O}_{2} 40 \%$ para eliminação da matéria orgânica.

Mantiveram-se as amostras na chapa aquecida em temperatura de aproximadamente $70^{\circ} \mathrm{C}$ até a secura e posteriormente foi feita a mudança do meio para clorídrico com a adição de $5 \mathrm{~mL}$ de $\mathrm{HCl} 6,25 \mathrm{~mol} \mathrm{~L}^{-1}$ e levado novamente a secura por três vezes, após foi elevado o volume com acréscimo de água super pura a um volume de $200 \mathrm{~mL}$.

Após o resfriamento da amostra adicionou-se $0,5 \mathrm{~g}$ de ácido ascórbico $\mathrm{e}$ o ajustou o pH na faixa de 1,5 a 2,0 com auxílio de $\mathrm{HCl}$ ou $\mathrm{NH}_{4} \mathrm{OH}$. Na solução foi 
colocada a cela de deposição com o disco de cobre previamente polido e limpo e levado a agitação continua e temperatura de aproximadamente $70^{\circ} \mathrm{C}$ por 4 horas para a deposição espontânea dos dois isótopos de polônios, FIG.3.11.

FIGURA 3.11 - Deposição espontânea de ${ }^{209} \mathrm{Po}$ e ${ }^{210} \mathrm{Po}$ sob agitação.

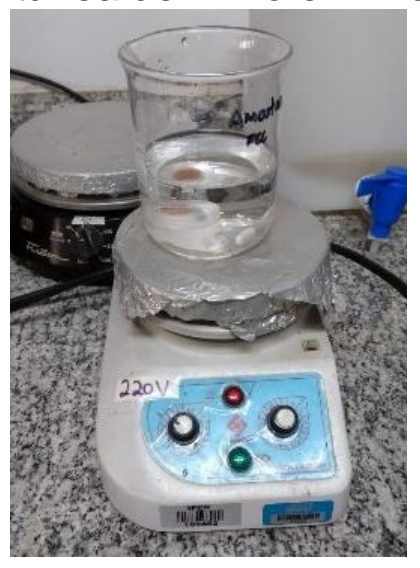

Após a deposição o disco foi retirado, lavado com água superpura e seco em chapa aquecedora a $70^{\circ} \mathrm{C}, \mathrm{FIG}$. 3.12.

FIGURA $3.12-{ }^{209} \mathrm{Po}$ e ${ }^{210} \mathrm{Po}$ depositados

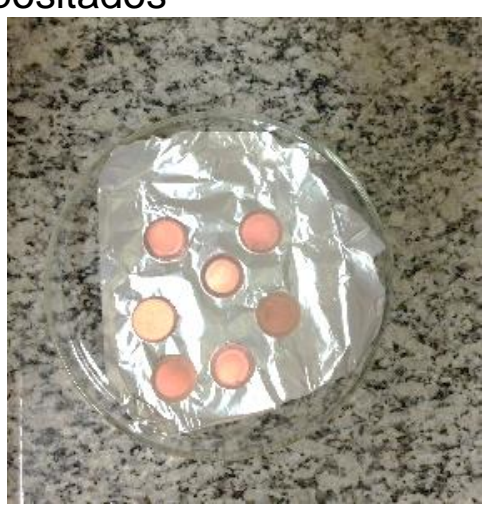

A medida da atividade foi feita em um espectrômetro alfa com detector de barreira de superfície por 150.000 segundos da marca Camberra, FIG.3.13. 
FIGURA 3.13 - Espectrômetro alfa



FIGURA 3.14 - Espectro obtido na análise de ${ }^{210} \mathrm{Po}$

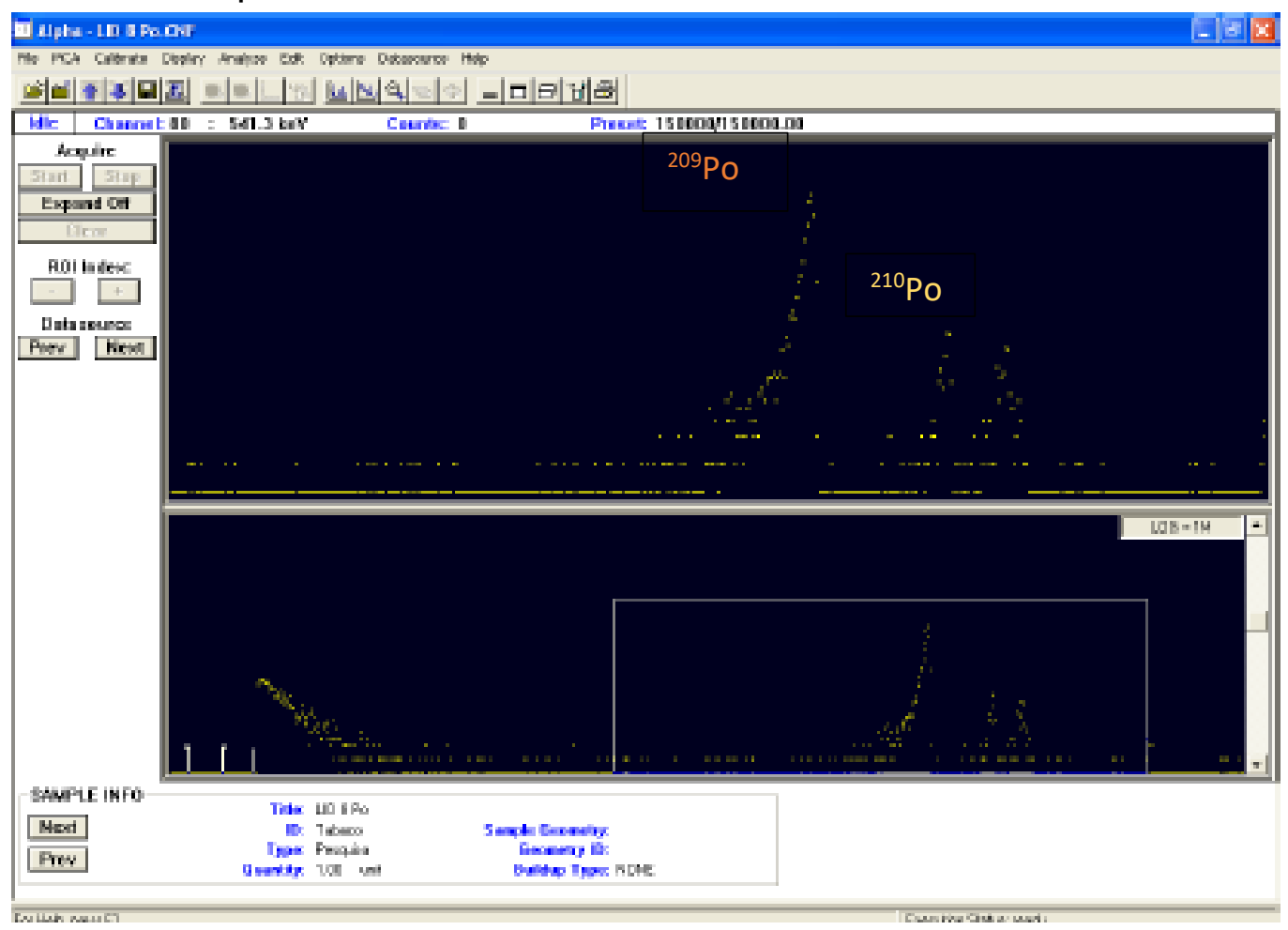

O cálculo da concentração de atividade do ${ }^{210} \mathrm{Po}$ foi determinado pela expressão (3.5).

$$
A^{210} P o=\frac{c p s a \cdot m t \cdot A t}{c p s t \cdot m a}
$$

Em que:

$\mathrm{A}^{210} \mathrm{Po}=$ concentração de atividade ${ }^{210} \mathrm{Po}$ na amostra em $\mathrm{mBq} \mathrm{g}^{-1}$

At $=$ atividade do traçador de ${ }^{209} \mathrm{Po} \mathrm{mBq} \mathrm{g}^{-1}$ 
cpsa $=$ taxa de contagem total da amostra em cps

cpst $=$ taxa de contagem do traçador de ${ }^{209} \mathrm{Po}$ em cps

$\mathrm{mt}=$ massa do traçador em $\mathrm{g}$

$\mathrm{ma}=$ massa da amostra em $\mathrm{g}$

\subsection{Avaliação de Dose}

Para o cálculo da dose anual estimada foi levado em consideração que um cigarro tem aproximadamente $0,5 \mathrm{~g}$ de tabaco e que um fumante consome um maço de cigarro por dia contendo 20 cigarros, portanto uma massa de $10 \mathrm{~g}$ de tabaco por dia ou $3,65 \mathrm{~kg}$ por ano. As frações ingeridas dos radionuclídeos estudados, da fumaça para os pulmões, seguiram os indicados na referência bibliográfica atual: ${ }^{210} \mathrm{Po}$ de 0,5 (TAHIR, 2008) e de ${ }^{210} \mathrm{~Pb}$ de 0,2 (BEGY, 2015). Para o cálculo da dose anual estimada oi utilizada a seguinte expressão (3.6):

$$
D=M a \times A \times F C D \times F I
$$

Em que:

D: Dose anual estimada em Sv ano-1;

Ma: massa de tabaco consumida por ano em $\mathrm{kg}$;

A: concentração de atividade média calculada em $\mathrm{mBq} \mathrm{g}^{-1}$

FCD: fator de conversão de dose para o radionuclídeo em Sv Bq-1;

FI: fração ingerida do radionuclídeo

Os valores de FCD foram obtidos da Publicação 119 do ICRP (International Committe Radiological Protection), 1,0 x 10-6 $\mathrm{Sv} \mathrm{Bq}^{-1}$ para o ${ }^{210} \mathrm{~Pb}$ e de $3,3 \times 10^{-1} \mathrm{~Sv} \mathrm{~Bq}^{-1}$ para o ${ }^{210} \mathrm{Po}$ (ICRP, 2013).

Para o cálculo da dose anual efetiva ( $\left.D_{A}\right)$ foi usada a expressão (3.7)

$$
D_{A}=D_{P o}+D_{P b}
$$

Em que:

$D_{A}$ : Dose anual efetiva em $\mu \mathrm{Sv}$

Dpo: Dose efetiva para o radionuclídeo ${ }^{210} \mathrm{Po}$ em $\mu \mathrm{Sv}$

Dpb: Dose efetiva para o radionuclídeo ${ }^{210} \mathrm{~Pb}$ em $\mu \mathrm{Sv}$ 


\section{RESULTADOS E DISCUSSÃO}

\subsection{Análise por Ativação com Nêutrons Instrumental - INAA}

\subsubsection{Precisão e exatidão da INAA}

Para verificação da precisão e exatidão da metodologia foram utilizados os materiais de referencia (Soil 1, Montanna II e Tomato Leaves), calculando-se a concentração de um padrão em função dos outros dois: Soil 1 em função do Montanna II e Tomato Leaves; Montanna II em função do Soil 1 e Tomato Leaves; e Tomato Leaves em função do Soil 1 e Montanna II. Os cálculos foram realizados em dez medidas para cada padrão.

Nas TAB. 4.1, 4.2 e 4.3 são apresentados os valores de concentração para os elementos determinados nos materiais de referência analisados. A precisão do metodo variou 2 a 20\% para o Soil 1, de 2 a 20\% para o Montanna II e 3 a 18\% para o Tomato Leaves. A exatidão do método variou de 2 a 28\% para o Soil 1, 1 a 17\% para o Montanna II e 1 a 13\% para o Tomato Leaves.

Calculou-se o erro relativo (ER) das medidas para avaliar a exatidão e o desvio padrão relativo (DPR) para avaliar a precisão do método, seguindo as expressões, 4.1 e 4.2 respectivamente.

$$
E R=\frac{\text { Ci-Ciref }}{\text { Ciref }} X 100
$$

Em que

- ER: erro relativo expresso em porcentagem;

- Ci: concentração do elemento de interesse medido no padrão de referência;

- Ciref: valor de referência do elemento de interesse no padrão.

$$
D P R=\frac{\mathrm{S}}{\bar{x}} \cdot 100
$$

Em que:

DPR: desvio padrão relativo em \%

S: desvio padrão dos resultados de concentração material de referência;

$\mathrm{x}$ : media dos resultados de concentração do material de referência 
TABELA 4.1 - Concentrações médias calculadas e concentrações certificadas, em $\mathrm{mg} \mathrm{kg}^{-1}$, para o material de referência Soil 1 utilizando Montanna II e Tomato Leaves como padrões.

\begin{tabular}{|c|c|c|c|c|}
\hline Elemento & Valor Calculado & $\begin{array}{c}\text { Valor } \\
\text { Certificado }\end{array}$ & DPR & ER \\
\hline As & $30 \pm 2$ & $27,6 \pm 2,9$ & 13 & 10 \\
\hline $\mathrm{Ba}$ & $666 \pm 82$ & $639 \pm 53$ & 20 & 4 \\
\hline $\mathrm{Ce}$ & $97 \pm 8$ & $117 \pm 17$ & 14 & 17 \\
\hline Co & $16,7 \pm 0,5$ & $19,8 \pm 1,5$ & 12 & 16 \\
\hline $\mathrm{Cr}$ & $100 \pm 9$ & 104 & 18 & 4 \\
\hline Cs & $6,0 \pm 0,4$ & 7,0 & 10 & 14 \\
\hline Eu & $1,6 \pm 0,3$ & 1,6 & 13 & 7 \\
\hline $\mathrm{Fe}$ & $64103 \pm 2033$ & $67400 \pm 1700$ & 14 & 5 \\
\hline $\mathrm{Hf}$ & $4,7 \pm 0,5$ & 4,2 & 8 & 13 \\
\hline $\mathbf{K}$ & $14985 \pm 2065$ & 14500 & 15 & 3 \\
\hline La & $49 \pm 2$ & $52,6 \pm 3,1$ & 11 & 7 \\
\hline Lu & $0,50 \pm 0,02$ & 0,54 & 14 & 8 \\
\hline $\mathrm{Na}$ & $1616 \pm 101$ & 1700 & 12 & 5 \\
\hline $\mathbf{R b}$ & $115 \pm 9$ & $113 \pm 11$ & 14 & 2 \\
\hline Sb & $1,3 \pm 0,2$ & 1,31 & 18 & 2 \\
\hline Sc & $16,0 \pm 0,3$ & 17,3 & 5 & 7 \\
\hline Sm & $8,0 \pm 0,3$ & 9,25 & 14 & 14 \\
\hline $\mathrm{Tb}$ & $1,3 \pm 0,2$ & 1,4 & 13 & 8 \\
\hline Th & $14 \pm 2$ & $14 \pm 1$ & 12 & 2 \\
\hline $\mathbf{U}$ & $2,9 \pm 1,1$ & 4,02 & 12 & 28 \\
\hline $\mathrm{Yb}$ & $3,0 \pm 0,1$ & 3,42 & 10 & 13 \\
\hline $\mathrm{Zn}$ & $229 \pm 9$ & $223 \pm 20$ & 2 & 2 \\
\hline
\end{tabular}

DPR $=$ Desvio padrão relativo e ER $=$ erro relativo. Os valores apresentados sem os respectivos desvios padrões correspondem a valores informativos. 
TABELA 4.2 - Concentrações médias calculadas e concentrações certificadas, em $\mathrm{mg} \mathrm{kg}^{-1}$, para o material de referência Montanna II utilizando Soil 1 e Tomato Leaves como padrões.

\begin{tabular}{|c|c|c|c|c|}
\hline Elemento & $\begin{array}{c}\text { Valor } \\
\text { Calculado }\end{array}$ & $\begin{array}{c}\text { Valor } \\
\text { Certificado }\end{array}$ & DPR & ER \\
\hline As & $101 \pm 11$ & $107 \pm 5$ & 10 & 6 \\
\hline $\mathrm{Ba}$ & $720 \pm 99$ & $730 \pm 15$ & 15 & 5 \\
\hline $\mathrm{Ca}$ & $29707 \pm 3495$ & $24200 \pm 600$ & 4 & 12 \\
\hline $\mathrm{Ce}$ & $77 \pm 6$ & 70 & 11 & 9 \\
\hline Co & $9,1 \pm 0,3$ & $9,89 \pm 0,18$ & 8 & 7 \\
\hline $\mathrm{Cr}$ & $49 \pm 5$ & $52,3 \pm 2,9$ & 2 & 6 \\
\hline Cs & $6,8 \pm 0,9$ & $6,7 \pm 0,2$ & 12 & 2 \\
\hline Eu & $1,0 \pm 0,3$ & 1,1 & 12 & 10 \\
\hline $\mathrm{Fe}$ & $29488 \pm 826$ & $28200 \pm 400$ & 6 & 4 \\
\hline Hf & $9,1 \pm 0,7$ & 9,2 & 15 & 2 \\
\hline K & $26518 \pm 4400$ & $25300 \pm 1000$ & 12 & 5 \\
\hline La & $40 \pm 2$ & 38 & 4 & 5 \\
\hline Lu & $0,5 \pm 0,1$ & 0,5 & 17 & 7 \\
\hline $\mathrm{Na}$ & $11123 \pm 782$ & $12000 \pm 100$ & 16 & 7 \\
\hline $\mathbf{R b}$ & $126 \pm 11$ & $120 \pm 3$ & 19 & 5 \\
\hline Sb & $24 \pm 2$ & $23,8 \pm 1,4$ & 13 & 1 \\
\hline Sc & $8,4 \pm 0,3$ & 8,5 & 20 & 1 \\
\hline Sm & $6,0 \pm 0,3$ & $5,93 \pm 0,28$ & 12 & 1 \\
\hline $\mathrm{Tb}$ & $0,9 \pm 0,1$ & 0,8 & 13 & 17 \\
\hline Th & $15 \pm 2$ & $15 \pm 1$ & 12 & 1 \\
\hline $\mathbf{U}$ & $3,4 \pm 0,2$ & $3,01 \pm 0,12$ & 14 & 11 \\
\hline Yb & $3,2 \pm 0,2$ & 3 & 9 & 6 \\
\hline $\mathbf{Z n}$ & $423 \pm 11$ & $414 \pm 11$ & 5 & 4 \\
\hline
\end{tabular}

DPR = Desvio padrão relativo e ER = erro relativo. Os valores apresentados sem os respectivos desvios padrões correspondem a valores informativos. 
TABELA 4.3 - Concentrações médias calculadas e concentrações certificadas, em $\mathrm{mg} \mathrm{kg}^{-1}$, para o material de referência Tomato Leaves utilizando Soil 1 e Montanna Il como padrões.

\begin{tabular}{ccccc}
\hline & Valor Calculado & Valor Certificado & DPR & ER \\
\hline Ba & $66 \pm 10$ & 63 & 8 & 4 \\
\hline $\mathbf{C a}$ & $50770 \pm 660$ & $50500 \pm 900$ & 3 & 1 \\
\hline $\mathbf{C e}$ & $1,9 \pm 0,3$ & 2 & 17 & 7 \\
\hline $\mathbf{C o}$ & $0,64 \pm 0,04$ & $0,57 \pm 0,02$ & 5 & 13 \\
\hline $\mathbf{C r}$ & $2,0 \pm 0,2$ & $1,99 \pm 0,006$ & 10 & 1 \\
\hline $\mathbf{F e}$ & $366 \pm 19$ & $368 \pm 7$ & 3 & 1 \\
\hline $\mathbf{H f}$ & $0,14 \pm 0,02$ & 0,14 & 9 & 1 \\
\hline $\mathbf{K}$ & $27545 \pm 5429$ & $27000 \pm 500$ & 5 & 2 \\
\hline $\mathbf{L a}$ & $2,2 \pm 0,1$ & 2,3 & 13 & 5 \\
\hline $\mathbf{N a}$ & $141 \pm 13$ & $136 \pm 4$ & 8 & 3 \\
\hline $\mathbf{R b}$ & $14 \pm 1$ & $14,89 \pm 0,27$ & 18 & 7 \\
\hline Sc & $0,102 \pm 0,004$ & 0,1 & 7 & 2 \\
\hline Sm & $0,20 \pm 0,02$ & 0,19 & 10 & 7 \\
\hline Th & $0,12 \pm 0,03$ & 0,12 & 16 & 1 \\
\hline Zn & $27 \pm 2$ & $30,9 \pm 0,7$ & 9 & 11 \\
\hline DPR
\end{tabular}

DPR $=$ Desvio padrão relativo e ER $=$ erro relativo. Os valores apresentados sem os respectivos desvios padrões correspondem a valores informativos.

Foi utilizado o critério estatístico do Z-score para verificar os valores calculados com os valores referenciais para verificar a exatidão da metodologia. $O$ cálculo da diferença padronizada ou valor de Z-score (Z) (BODE,1996) foi calculado pela seguinte expressão (4.3):

$$
Z=\frac{C_{i}-C_{i r e f}}{S_{i}}
$$

Em que:

$\mathrm{C}_{\mathrm{i}}$ : concentração média do elemento de interesse medido na padrão de referência;

Ciref: valor de referência do elemento de interesse no padrão;

Si: desvio padrão do elemento de interesse medido no padrão de referência;

Usou-se a concentração média de dez medidas de cada padrão para os cálculos do Z-score. Os valores são apresentados nas FIG. 4.1, 4.2 e 4.3. 
Para | Z | $<2$ os resultados são considerados satisfatórios; $2<\mid \mathrm{Z}$ | $\leq 3$ os resultados são considerados questionáveis e I Z I > 3 os resultados são considerados insatisfatórios.

FIGURA 4.1 - Z score material de referência Soil 1 pela INAA.

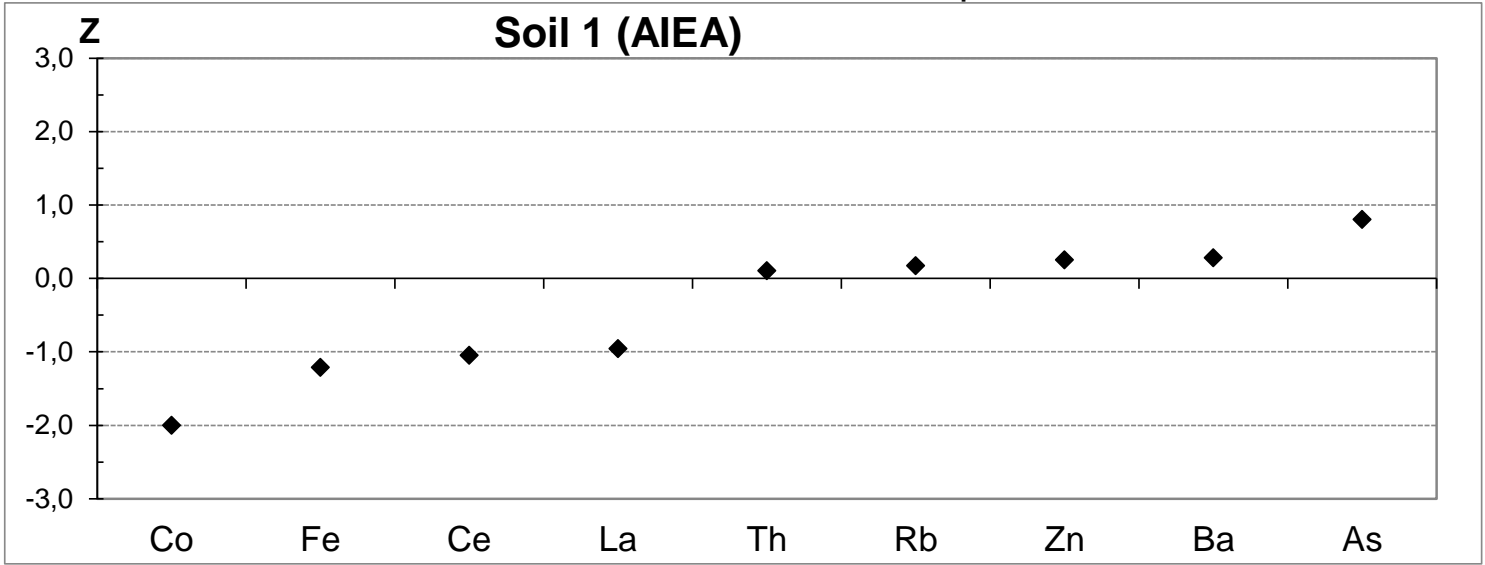

FIGURA 4.2 - Z score material de referência Montanna II pela INAA.

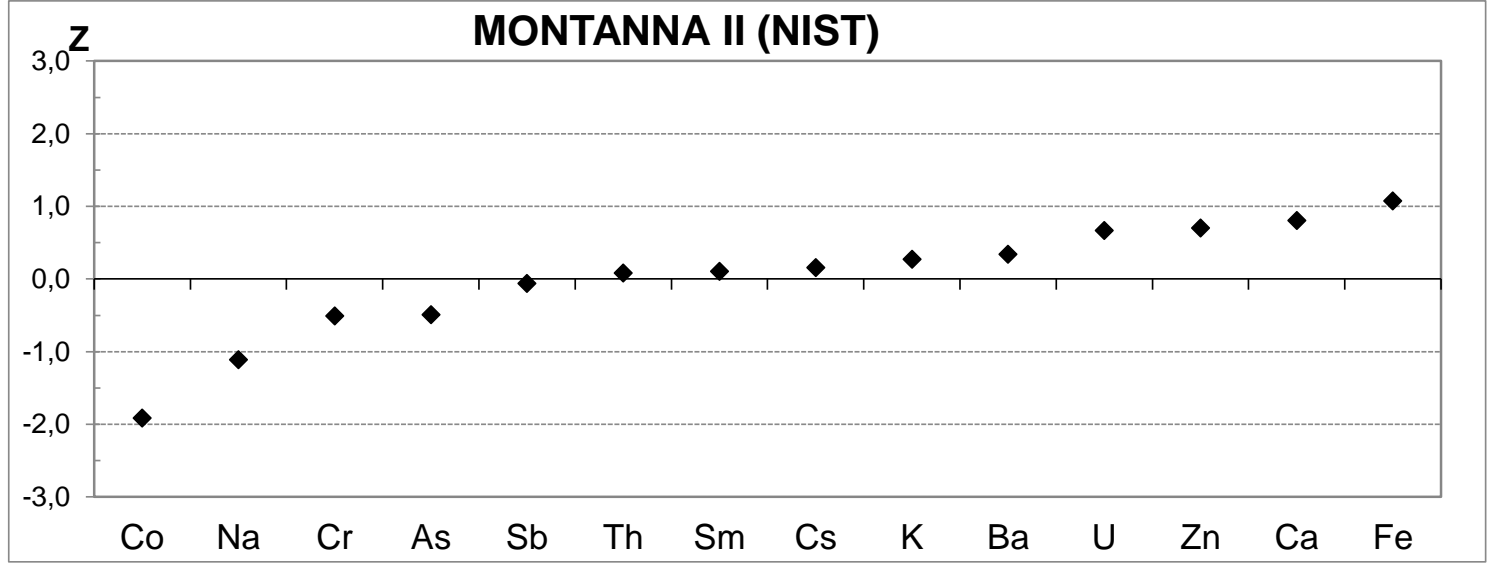

FIGURA 4.3 - Z score material de referência Tomato Leaves pela INAA.

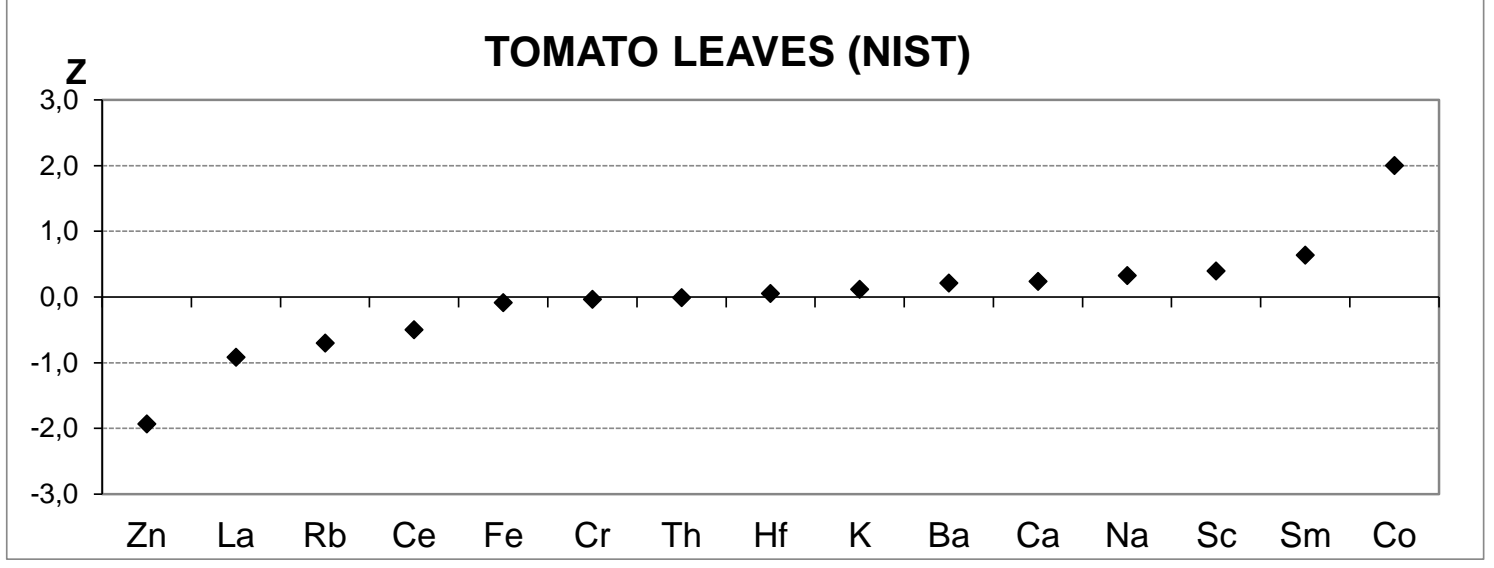




\subsubsection{Determinação do limite inferior de detecção (LID)}

Os limites de detecção para os elementos determinados por INAA foram calculados utilizando os materiais de referência SL1, Montanna II e Tomato Leaves, segunda a expressão (4.4)

$$
L I D=\frac{3 C_{r e f} \cdot \sqrt{B g}}{T \cdot C \cdot M}
$$

Em que:

LID: limite inferior de determinação $\left(\mathrm{mg} \mathrm{kg}^{-1}\right)$;

Cref: valor certificado do elemento no padrão $\left(\mathrm{mg} \mathrm{kg}^{-1}\right)$;

Bg: contagens da radiação de fundo do elemento analisado (contagens);

$\mathrm{T}$ : tempo de contagem (s);

C: contagens do elemento de interesse (cps)

M: massa da amostra.

Os valores obtidos são apresentados na TAB. 4.4. 
TABELA 4.4 - Limites inferiores de determinação para os elementos estudados por INAA, em mg kg-1, para os materiais de referência Soil 1, Montanna II e Tomato Leaves.

\begin{tabular}{|c|c|c|c|}
\hline Elementos & Soil1 & Montanna II & Tomato Leaves \\
\hline As & 1,4 & 1,8 & * \\
\hline $\mathrm{Ba}$ & 103 & 96 & 39 \\
\hline $\mathrm{Br}$ & - & - & 5,2 \\
\hline $\mathrm{Ca}$ & - & 7192 & 7147 \\
\hline $\mathrm{Ce}$ & 3,01 & 1,9 & 0,79 \\
\hline Co & 0,71 & 0,49 & 0,13 \\
\hline $\mathrm{Cr}$ & 4,42 & 2,9 & 0,82 \\
\hline Cs & 0,80 & - & 0,04 \\
\hline Eu & 0,09 & 0,09 & - \\
\hline $\mathrm{Fe}$ & 937 & 643 & 140 \\
\hline Hf & 0,29 & 0,34 & 0,09 \\
\hline K & 3860 & 3020 & 6388 \\
\hline La & 0,75 & 0,62 & 0,53 \\
\hline Lu & 0,05 & - & - \\
\hline $\mathrm{Na}$ & 19 & 41 & 44 \\
\hline Nd & - & 32 & - \\
\hline Rb & 13 & 11 & 4,5 \\
\hline Sb & 0,21 & 16 & * \\
\hline Sc & 0,13 & 0,95 & 0,01 \\
\hline $\mathrm{Se}$ & 0,41 & 0,29 & - \\
\hline Sm & 0,45 & 0,68 & 0,04 \\
\hline Ta & 0,47 & 0,22 & - \\
\hline Tb & 0,12 & 0,05 & - \\
\hline Th & 0,38 & 0,38 & * \\
\hline $\mathbf{U}$ & 0,64 & 0,48 & - \\
\hline $\mathrm{Yb}$ & 0,62 & 0,30 & - \\
\hline $\mathrm{Zn}$ & 13 & 18 & 5,3 \\
\hline
\end{tabular}

* não determinado

- não possui valores de referência do elemento na padrão

\subsection{Espectrometria de Fluorescência de Raios-X (EDXRF)}

\subsubsection{Precisão e exatidão da EDXRF}

A curva de calibração foi ajustada a partir das leituras dos padrões. Foram realizadas sete leituras para cada elemento analisado dos três padrões e calculado valores médios que foram comparados com os valores certificados dos padrões. 
Para verificar a precisão das medidas foi aplicado o desvio padrão relativo (DPR), o qual é uma medida normalizada de dispersão de uma distribuição de probabilidade ou de frequência, calculado pela expressão (4.5).

$$
D P R=\frac{\mathrm{S}}{\bar{x}} \cdot 100
$$

Em que:

DPR: desvio padrão relativo em \%

S: desvio padrão dos resultados de concentração material de referência;

x: média dos resultados de concentração do material de referência;

Também foi calculado o erro relativo (ER) das medidas para avaliar a exatidão do método, seguindo a expressão (4.6):

$$
E R=\frac{\text { Ci-Ciref }}{\text { Ciref }} X 100
$$

Em que

- ER: erro relativo expresso em porcentagem;

- Ci: concentração do elemento de interesse medido no padrão de referência;

- Ciref: valor de referência do elemento de interesse no padrão.

Nas TAB 4.5, 4.6 e 4.7 são apresentados as concentrações médias calculadas, as concentrações certificadas, os desvios padrões relativos (DPR) e os erros relativos (ER). A precisão do método variou 1 a 23\% para o Peach Leaves, de 1 a 12\% para o Sargasso e de 1 a 15\% para o Tomato Leaves. A exatidão do método variou de 1 a 28\% para o Peach Leaves, 1 a 13\% para o Sargasso e 1 a 15\% para o Tomato Leaves. 
TABELA 4.5 - Concentrações médias calculadas e concentrações certificadas, em $\mathrm{mg} \mathrm{kg}^{-1}(* 0)$, para o material de referência Peach Leaves.

\begin{tabular}{|c|c|c|c|c|}
\hline Elemento & Valor Certificado & Valor Calculado & DPR & ER \\
\hline Al & $249 \pm 8$ & $226 \pm 22$ & 10 & 9 \\
\hline As & $0,06 \pm 0,018$ & $0,0592 \pm 0,0004$ & 1 & 1 \\
\hline $\mathbf{B a}$ & $124 \pm 4$ & $124 \pm 11$ & 9 & 1 \\
\hline $\mathrm{Br}$ & 11 & $11 \pm 1$ & 9 & 1 \\
\hline${ }^{\star} \mathrm{Ca}$ & $1,56 \pm 0,02$ & $1,53 \pm 0,03$ & 2 & 2 \\
\hline $\mathrm{Ce}$ & 10 & $11 \pm 2$ & 9 & 5 \\
\hline $\mathrm{Cl}$ & $360 \pm 19$ & $352 \pm 25$ & 7 & 2 \\
\hline Co & 0,07 & $0,062 \pm 0,004$ & 5 & 11 \\
\hline $\mathrm{Cr}$ & 1 & $1,0 \pm 0,1$ & 8 & 1 \\
\hline $\mathrm{Cu}$ & $3,7 \pm 0,4$ & $3,8 \pm 0,3$ & 9 & 2 \\
\hline $\mathbf{F e}$ & 220 & $224 \pm 4$ & 2 & 3 \\
\hline $\mathrm{Hg}$ & $0,031 \pm 0,007$ & $0,032 \pm 0,001$ & 2 & 2 \\
\hline${ }^{\star} \mathbf{K}$ & $2,43 \pm 0,03$ & $2,37 \pm 0,13$ & 5 & 3 \\
\hline La & 9 & $9,5 \pm 0,8$ & 8 & 5 \\
\hline${ }^{\star} \mathrm{Mg}$ & $0,432 \pm 0,008$ & $0,42 \pm 0,01$ & 2 & 3 \\
\hline Mn & $98 \pm 3$ & $99 \pm 5$ & 5 & 1 \\
\hline Nd & 7 & $8,6 \pm 0,3$ & 3 & 22 \\
\hline $\mathbf{N i}$ & $0,69 \pm 0,09$ & $0,84 \pm 0,2$ & 6 & 22 \\
\hline $\mathbf{P b}$ & $0,87 \pm 0,03$ & $0,75 \pm 0,13$ & 15 & 10 \\
\hline $\mathbf{R b}$ & 19 & $20,0 \pm 0,2$ & 1 & 1 \\
\hline${ }^{*} S$ & 0,2 & $0,190 \pm 0,001$ & 1 & 5 \\
\hline $\mathrm{Sb}$ & 0,02 & $0,0208 \pm 0,0004$ & 2 & 4 \\
\hline Se & $0,120 \pm 0,009$ & $0,10 \pm 0,01$ & 7 & 14 \\
\hline Sm & 1 & $1,1 \pm 0,1$ & 14 & 7 \\
\hline $\mathrm{Sr}$ & $53 \pm 4$ & $49 \pm 1$ & 1 & 7 \\
\hline Th & 0,05 & $0,064 \pm 0,001$ & 2 & 28 \\
\hline V & $0,37 \pm 0,03$ & $0,34 \pm 0,08$ & 23 & 7 \\
\hline $\mathrm{Zn}$ & $17,9 \pm 0,4$ & $17,8 \pm 0,2$ & 1 & 1 \\
\hline
\end{tabular}

Os valores apresentados sem os respectivos desvios padrões correspondem a valores informativos 
TABELA 4.6 - Concentrações médias calculadas e concentrações certificadas, em $\mathrm{mg} \mathrm{kg}^{-1}\left({ }^{*} \%\right)$, para o material de referência Sargasso.

\begin{tabular}{|c|c|c|c|c|}
\hline Elemento & $\begin{array}{c}\text { Valor } \\
\text { Certificado }\end{array}$ & Valor Calculado & DPR & ER \\
\hline Al & 215 & $206 \pm 28$ & 4 & 4 \\
\hline $\mathrm{Br}$ & 270 & $270 \pm 6$ & 2 & 1 \\
\hline${ }^{\star} \mathrm{Ca}$ & $1,34 \pm 0,05$ & $1,26 \pm 0,03$ & 2 & 6 \\
\hline${ }^{*} \mathrm{Cl}$ & 5,1 & $4,8 \pm 0,1$ & 2 & 6 \\
\hline Co & $0,12 \pm 0,01$ & $0,11 \pm 0,04$ & 6 & 8 \\
\hline $\mathrm{Cr}$ & 0,2 & $0,21 \pm 0,02$ & 10 & 4 \\
\hline Cs & 0,04 & $0,041 \pm 0,001$ & 1 & 3 \\
\hline $\mathrm{Cu}$ & $4,9 \pm 0,2$ & $4,7 \pm 0,1$ & 3 & 4 \\
\hline $\mathrm{Fe}$ & $187 \pm 6$ & $196 \pm 11$ & 2 & 5 \\
\hline $\mathrm{Hg}$ & 0,04 & $0,0406 \pm 0,0005$ & 1 & 2 \\
\hline I & 520 & $501 \pm 37$ & 7 & 4 \\
\hline${ }^{\star} \mathrm{K}$ & $6,10 \pm 0,20$ & $0,63 \pm 0,04$ & 6 & 2 \\
\hline${ }^{\star} \mathrm{Mg}$ & $0,65 \pm 0,03$ & $0,58 \pm 0,02$ & 4 & 10 \\
\hline Mn & $21,2 \pm 1,0$ & $19 \pm 1$ & 9 & 10 \\
\hline${ }^{\star} \mathbf{P}$ & 0,26 & $0,24 \pm 0,01$ & 5 & 8 \\
\hline $\mathbf{P b}$ & $1,35 \pm 0,05$ & $1,5 \pm 0,4$ & 7 & 8 \\
\hline $\mathbf{R b}$ & $24 \pm 2$ & $24 \pm 1$ & 4 & 2 \\
\hline${ }^{\star} \mathrm{S}$ & 1,2 & $1,05 \pm 0,002$ & 1 & 13 \\
\hline Sb & 0,04 & $0,037 \pm 0,001$ & 2 & 7 \\
\hline Se & 0,05 & $0,050 \pm 0,001$ & 1 & 1 \\
\hline $\mathrm{Sr}$ & $1000 \pm 30$ & $980 \pm 67$ & 7 & 2 \\
\hline $\mathbf{U}$ & 0,4 & $0,43 \pm 0,09$ & 12 & 7 \\
\hline V & $1,0 \pm 0,1$ & $0,9 \pm 0,2$ & 8 & 1 \\
\hline $\mathrm{Zn}$ & $15,6 \pm 1,2$ & $16,2 \pm 0,4$ & 3 & 4 \\
\hline
\end{tabular}

Os valores apresentados sem os respectivos desvios padrões correspondem a valores informativos 
TABELA 4.7 - Concentrações médias calculadas e concentrações certificadas, em $\mathrm{mg} \mathrm{kg}^{-1}\left({ }^{*} \%\right)$, para o material de referência Tomato Leaves.

\begin{tabular}{|c|c|c|c|c|}
\hline Elemento & $\begin{array}{c}\text { Valor } \\
\text { Certificado }\end{array}$ & Valor Calculado & DPR & ER \\
\hline Al & $598 \pm 12$ & $583 \pm 33$ & 6 & 3 \\
\hline As & $0,112 \pm 0,004$ & $0,103 \pm 0,015$ & 14 & 8 \\
\hline $\mathrm{Ba}$ & 63 & $54 \pm 3$ & 6 & 14 \\
\hline $\mathrm{Br}$ & 1300 & $1390 \pm 234$ & 10 & 7 \\
\hline${ }^{\star} \mathrm{Ca}$ & $5,05 \pm 0,09$ & $5,2 \pm 0,3$ & 5 & 6 \\
\hline Cd & $1,52 \pm 0,04$ & $1,4 \pm 0,2$ & 15 & 5 \\
\hline $\mathrm{Cl}$ & 6600 & $0,56 \pm 0,06$ & 11 & 15 \\
\hline Co & $0,57 \pm 0,02$ & $0,53 \pm 0,02$ & 4 & 6 \\
\hline $\mathrm{Cr}$ & $1,99 \pm 0,006$ & $1,99 \pm 0,2$ & 10 & 5 \\
\hline Cs & 0,053 & $0,056 \pm 0,003$ & 5 & 5 \\
\hline $\mathrm{Cu}$ & $4,70 \pm 0,14$ & $4,9 \pm 0,4$ & 8 & 5 \\
\hline $\mathrm{Fe}$ & $368 \pm 7$ & $350 \pm 18$ & 3 & 5 \\
\hline $\mathrm{Hg}$ & $0,034 \pm 0,004$ & $0,034 \pm 0,001$ & 3 & 3 \\
\hline I & 0,85 & $0,82 \pm 0,06$ & 8 & 4 \\
\hline${ }^{\star} \mathrm{K}$ & $2,7 \pm 0,05$ & $2,7 \pm 0,2$ & 6 & 1 \\
\hline La & 2,3 & $2,6 \pm 0,3$ & 11 & 13 \\
\hline${ }^{*} \mathrm{Mg}$ & 1,2 & $1,3 \pm 0,1$ & 6 & 9 \\
\hline Mn & $246 \pm 8$ & $243 \pm 10$ & 4 & 1 \\
\hline $\mathbf{N i}$ & $1,59 \pm 0,07$ & $1,48 \pm 0,16$ & 11 & 7 \\
\hline${ }^{*} \mathbf{P}$ & $0,216 \pm 0,004$ & $0,21 \pm 0,01$ & 3 & 3 \\
\hline $\mathbf{R b}$ & $14,89 \pm 0,27$ & $14 \pm 1$ & 6 & 7 \\
\hline${ }^{*} S$ & 0,96 & $0,856 \pm 0,005$ & 1 & 11 \\
\hline $\mathbf{S b}$ & $0,063 \pm 0,006$ & $0,065 \pm 0,008$ & 12 & 3 \\
\hline $\mathrm{Se}$ & $0,054 \pm 0,003$ & $0,054 \pm 0,001$ & 1 & 1 \\
\hline $\mathrm{Sr}$ & 85 & $84 \pm 1$ & 1 & 1 \\
\hline Th & 0,12 & $0,12 \pm 0,02$ & 14 & 1 \\
\hline V & $0,835 \pm 0,010$ & $0,81 \pm 0,06$ & 7 & 4 \\
\hline Zn & $30,9 \pm 0,7$ & $31 \pm 1$ & 9 & 1 \\
\hline
\end{tabular}

Os valores apresentados sem os respectivos desvios padrões correspondem a valores informativos

Também foram calculados os valores de Z score para cada elemento de cada padrão seguindo a expressão (4.7).

$$
Z=\frac{\left(X_{\text {calc }}-X_{\text {ref }}\right)}{S_{\text {ref }}}
$$


Em que:

Z: Z score

Xcalc: concentração média do material de referência calculado

Xref: concentração certificada do material de referência

Sref: desvio padrão das medidas de concentração

Nas FIG. 4.4, 4.5 e 4.6 são apresentados os valores de Z score determinados para os elementos analisados nos materiais de referência Peach Leaves, Sargasso e Tomato Leaves utilizados na técnica de EDXRF respectivamente.

\subsubsection{Determinação do limite de determinação do método (LDM)}

Para verificar os valores mínimos de concentração dos elementos avaliados pela técnica de EDXRF, foi calculado o limite de determinação do método (LDM) utilizando a expressão (4.8):

$$
L D M=2 \cdot \sqrt{\frac{\sum_{i=l}^{N}\left(C_{i}-C^{-}\right)^{2}}{N-1}}
$$

Em que:

LDM: Limite de determinação do Método

$\mathrm{C}_{\mathrm{i}:}$ concentração do material de referência calculado

C:: média das concentrações do material de referência calculado

$\mathrm{N}$ : número de repetições

Os valores obtidos de LDM são apresentados na TAB 4.8. 
FIGURA 4.4 - Z-score material de referência Peach Leaves pela técnica EDXRF.

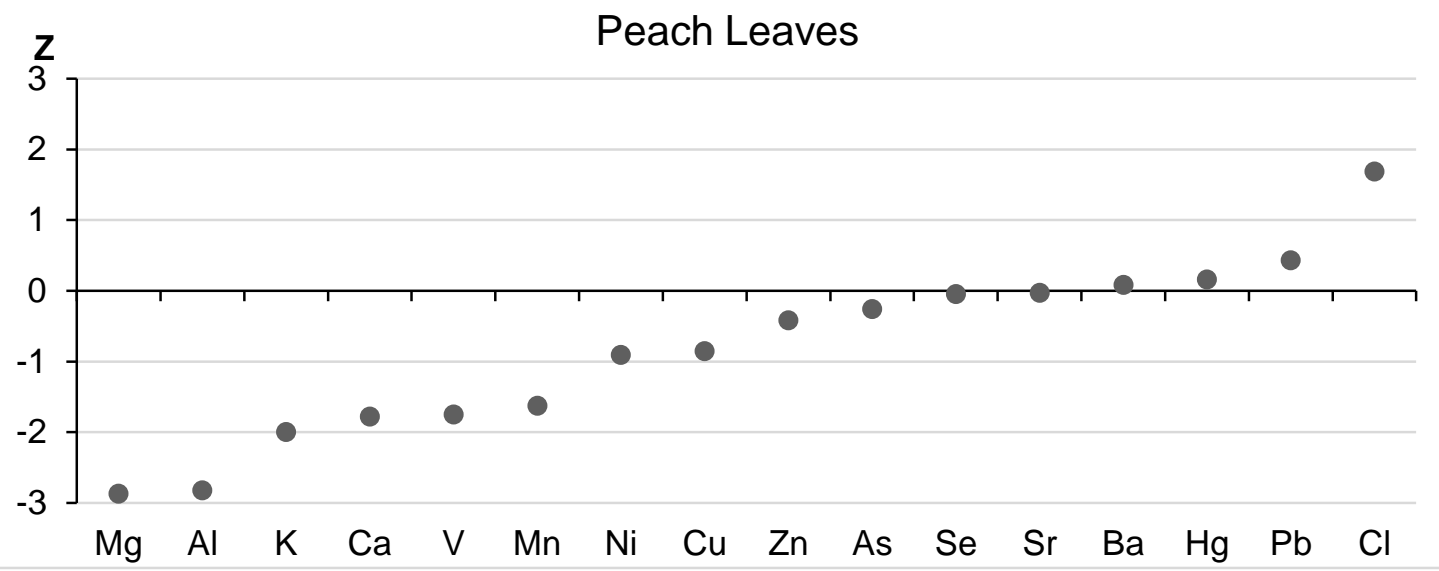

FIGURA 4.5 - Z-score material de referência Sargasso pela técnica EDXRF.

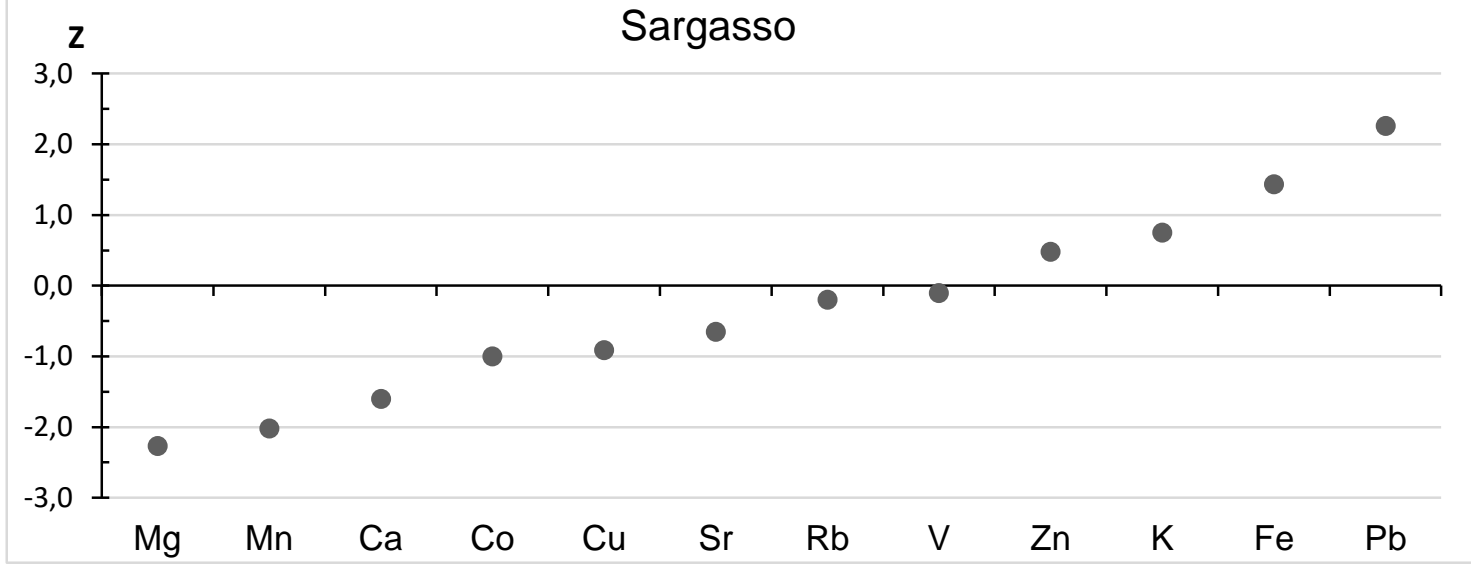

FIGURA 4.6 - Z-score material de referência Tomato Leaves pela técnica EDXRF.

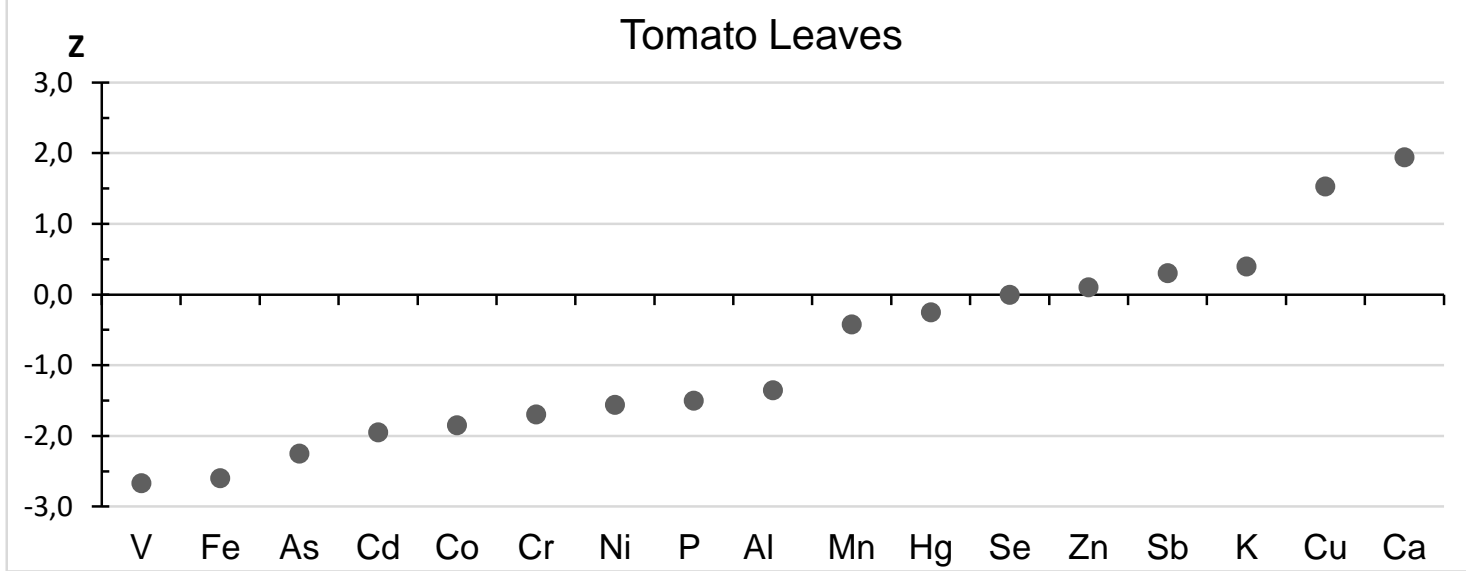


TABELA 4.8 - Limites de determinação do método para os elementos estudados por EDXRF, em mg kg-1 (* em \%), para os materiais de referencia Peach Leaves, Sargasso e Tomato Leaves.

\begin{tabular}{|c|c|c|c|}
\hline Elemento & Peach Leaves & Sargasso & Tomato Leaves \\
\hline Al & 70 & 67 & 106 \\
\hline As & 0,001 & - & 0,017 \\
\hline $\mathrm{Ba}$ & 41,4 & - & 29 \\
\hline $\mathrm{Br}$ & 3,4 & 99 & 751 \\
\hline${ }^{*} \mathrm{Ca}$ & 0,095 & 0,06 & 0,85 \\
\hline Cd & 0,39 & 0,21 & 1,4 \\
\hline $\mathrm{Cl}$ & 240 & 0,35 & 1177 \\
\hline Co & 0,053 & 0,09 & 0,18 \\
\hline $\mathrm{Cr}$ & 0,1 & 0,19 & 0,51 \\
\hline Cs & - & 0,001 & 0,02 \\
\hline $\mathrm{Cu}$ & 0,77 & 0,3 & 0,7 \\
\hline $\mathrm{Fe}$ & 41,4 & 31,6 & 26,8 \\
\hline $\mathrm{Hg}$ & 0,003 & 0,002 & 0,004 \\
\hline I & - & 68 & - \\
\hline${ }^{*} \mathrm{~K}$ & 0,22 & 0,6 & 0,61 \\
\hline La & 7,02 & - & 1,4 \\
\hline${ }^{\star} \mathrm{Mg}$ & 0,04 & 0,11 & 0,27 \\
\hline$M n$ & 92,9 & 10,6 & 88,1 \\
\hline Nd & 5,02 & - & - \\
\hline $\mathrm{Ni}$ & 0,61 & - & 0,58 \\
\hline${ }^{*} \mathbf{P}$ & - & 0,025 & 0,04 \\
\hline $\mathbf{P b}$ & 0,32 & 1,18 & - \\
\hline $\mathbf{R b}$ & 0,52 & 1,62 & 2,55 \\
\hline *S & 0,013 & 0,07 & 0,13 \\
\hline Sb & 0,019 & 0,004 & 0,2 \\
\hline $\mathrm{Se}$ & 0,03 & 0,04 & 0,0023 \\
\hline Sm & 0,87 & - & - \\
\hline $\mathrm{Sr}$ & 0,65 & 105 & 11,2 \\
\hline Th & 0,036 & - & 0,05 \\
\hline $\mathbf{U}$ & 0,098 & 0,13 & - \\
\hline V & 0,09 & 0,7 & 0,58 \\
\hline $\mathrm{Zn}$ & 2,41 & 2,2 & 5,4 \\
\hline
\end{tabular}

- não possui valores de referência do elemento no padrão

* concentrações em \% 


\subsection{Determinação da radiação de fundo}

Foi utilizado para determinação das atividades de concentração alfa e beta um detector de fluxo gasoso de baixa radiação de fundo da marca Berthold, modelo LB-770-2, com dez detectores e um contador de guarda em anticoincidência e foi medida as radiações de fundo da medida alfa e beta total por 200 minutos. Para a medida alfa o valor médio de radiação de fundo foi de 0,04 cpm e para a medida beta foi de 2,6 cpm.

A radiação de fundo no detector alfa na região dos picos de interesse dos radionuclídeos ${ }^{210} \mathrm{Po}$ e ${ }^{209} \mathrm{Po}$ foi determinada por um medida do detector vazio por tempo igual ao das amostras. Os valores obtidos para os para os isótopos de polônio foi de $2,5 \times 10^{-5} \mathrm{cps}$.

4.4 Determinação da eficiência de contagem para as medidas de ${ }^{226} \mathrm{Ra},{ }^{228} \mathrm{Ra}$ $e^{210} \mathrm{~Pb}$

As eficiências de contagem do detector proporcional para os radionuclídeos ${ }^{226} \mathrm{Ra}$ e ${ }^{228} \mathrm{Ra}$ foram realizadas de acordo com OLIVEIRA (1993) e para o radionuclídeo ${ }^{210} \mathrm{~Pb}$ foi realizada de acordo com MOREIRA (1993).

\subsubsection{Determinação da eficiência de contagem alfa total para a medida de ${ }^{226} \mathrm{Ra}$ (OLIVEIRA, 1993)}

Foi realizada a calibração do detector de fluxo gasoso utilizando-se uma fonte eletrodepositada de ${ }^{241} \mathrm{Am}$ que foi medida em triplicata em cada detector por 60 minutos. A eficiência foi calculada usando a expressão (4.9)

$$
E F \alpha\left({ }^{226} R a\right)=\frac{R n-B g}{A_{\text {corr }}\left({ }^{231} A m\right) \cdot 60 \cdot R Q}
$$

Em que:

$\mathrm{EF} \alpha\left({ }^{226} \mathrm{Ra}\right)$ : eficiência de contagem alfa para medida de ${ }^{226} \mathrm{Ra}$, calculada pelo padrão de ${ }^{241} \mathrm{Am}$ (cps/dps) 
Rn: taxa de contagem alfa medida (cpm)

Bg: radiação de fundo do detector alfa (cpm)

A corr $\left({ }^{241} \mathrm{Am}\right)$ : atividade da fonte de ${ }^{241} \mathrm{Am}(\mathrm{Bq})$

RQ: rendimento químico da eletrodeposição do padrão

O resultado médio da eficiência de contagem para a medida alfa do radionuclídeo ${ }^{226} \mathrm{Ra}$ foi de $24,2 \%$

\subsubsection{Determinação da eficiência de contagem beta de ${ }^{226}$ Ra para a medida de ${ }^{228} \mathrm{Ra}$}

A eficiência de contagem beta de ${ }^{226} \mathrm{Ra}$ para a medida de ${ }^{228} \mathrm{Ra}$ deve ser feita, pois no precipitado de $\mathrm{Ba}(\mathrm{Ra}) \mathrm{SO}_{4}$ estão presentes esses dois radionuclídeos. $\mathrm{Na}$ voltagem de operação são detectadas tanto partículas alfa emitidas pelo ${ }^{226} \mathrm{Ra}$ como partículas beta emitidas pelo seus descendentes e desta forma calcular o incremento na taxa de contagem beta total obtida na medida (OLIVEIRA,1993).

A eficiência de contagem beta do ${ }^{226} \mathrm{Ra}$ foi realizada com medidas beta total de amostras de $\mathrm{Ba}(\mathrm{Ra}) \mathrm{SO}_{4}$ onde foi adicionado um padrão de atividade conhecida de ${ }^{226} \mathrm{Ra}$. Para a determinação adicionou-se uma folha de papel filtro sobre o precipitado para bloquear as partículas beta de baixa energia de seus descendentes. Foram preparadas 5 amostras e medidas por 200 minutos. A eficiência foi calculada de acordo com a expressão (4.10).

$$
E F \beta\left({ }^{226} R a\right)=\frac{R n-B g}{A_{\text {corr }}\left({ }^{226} R a\right) \cdot R Q}
$$

Em que:

$\mathrm{EF} \beta\left({ }^{226} \mathrm{Ra}\right)$ : eficiência de contagem alfa para medida de ${ }^{226} \mathrm{Ra}$ (cps/dps)

$\mathrm{Rn}$ : taxa de contagem beta total medida (cpm)

$\mathrm{Bg}$ : radiação de fundo do detector (cpm)

$\left.A_{\text {corr }}{ }^{226} \mathrm{Ra}\right):$ atividade do padrão de ${ }^{226} \mathrm{Ra}(\mathrm{Bq})$

RQ: rendimento químico

O resultado médio da eficiência de contagem para a medida beta total do radionuclídeo ${ }^{226} \mathrm{Ra}$ foi de $51,5 \%$ 


\subsubsection{Determinação da eficiência de contagem beta de ${ }^{228} \mathrm{Ra}$}

A eficiência beta para o ${ }^{228} \mathrm{Ra}$ foi calculada a partir do precipitado de $\mathrm{Ba}(\mathrm{Ra}) \mathrm{SO} 4$ em amostras obtidas com adição de um padrão de atividade conhecida de ${ }^{228} \mathrm{Ra}$ e colocando um papel filtro sobre o precipitado para barrar as partículas beta de baixa energia. Foram preparadas 5 amostras e medidas por 200 minutos. A eficiência beta para o ${ }^{228} \mathrm{Ra}$ foi calculada de acordo com a expressão (4.11).

$$
E F \beta\left({ }^{228} R a\right)=\frac{R n-B g}{A_{c o r r}\left({ }^{228} R a\right) \cdot R Q}
$$

Em que:

$\mathrm{EF} \beta\left({ }^{228} \mathrm{Ra}\right)$ : eficiência de contagem alfa para medida de ${ }^{228} \mathrm{Ra}(\mathrm{cps} / \mathrm{dps})$

Rn: taxa de contagem beta total medida (cpm)

Bg: radiação de fundo do detector (cpm)

Acorr $\left({ }^{226} \mathrm{Ra}\right)$ : atividade do padrão de ${ }^{226} \mathrm{Ra}$ corrigida para a data da medida (Bq) RQ: rendimento químico

O resultado médio da eficiência de contagem para a medida beta total do radionuclídeo ${ }^{228} \mathrm{Ra}$ foi de $38,05 \%$.

\subsubsection{Determinação da eficiência de contagem beta de ${ }^{210} \mathrm{~Pb}$ (MOREIRA, 1993)}

A eficiência de contagem do ${ }^{210} \mathrm{~Pb}$ foi determinada utilizando um padrão de atividade conhecida deste radionuclídeo. A eficiência foi calculada de acordo com a expressão (4.12).

$$
E=\frac{R_{p}-B g}{A p \cdot R Q \cdot\left(1-e^{-\Lambda t}\right)}
$$

Em que:

E: eficiência de contagem do sistema (cps/dps)

$\mathrm{Rp}$ : taxa de contagem do padrão (cps)

Bg: radiação de fundo do detector (cps)

$A_{p}$ : atividade do padrão $(\mathrm{Bq})$

$\mathrm{RQ}$ : rendimento químico 
$\lambda$ : constante de desintegração do ${ }^{210} \mathrm{Bi}\left(0,183 d^{-1}\right)$

t: tempo transcorrido entre a precipitação e a medida (d)

O resultado médio da eficiência de contagem para a medida beta total do radionuclídeo ${ }^{210} \mathrm{~Pb}$ foi de $37,3 \%$.

\subsubsection{Determinação da eficiência do espectrômetro alfa}

A determinação da eficiência do espectrômetro alfa foi realizada segundo Alencar (2008) e Ramos (2010) utilizando uma fonte eletrodepositada de ${ }^{241} \mathrm{Am}$. Essa fonte foi medida por 100 segundos em triplicata, e o cálculo da eficiência foi efetuado de acordo com a seguinte expressão (4.13).

$$
E f=\frac{C f}{A f}
$$

Em que:

$E f=$ eficiência de contagem $\left(\mathrm{cps} \mathrm{dps}^{-1}\right)$

$C f=$ contagem da fonte (cps)

$A f=$ atividade da fonte $(\mathrm{dps})$

O resultado médio encontrado foi de $45,7 \%$.

\subsubsection{Determinação da curva de calibração do espectrômetro alfa}

A curva de calibração do espectrômetro alfa foi determinada segundo Alencar (2008) e Ramos (2010). Foi utilizada uma fonte tríplice da Amersham International, contendo os radionuclídeos ${ }^{239} \mathrm{Pu}(5,156 \mathrm{MeV}),{ }^{241} \mathrm{Am}(5,485 \mathrm{MeV}) \mathrm{e}$ ${ }^{244} \mathrm{Cm}(5,804 \mathrm{MeV})$, que foi medida por 100 segundos e em triplicata.

4.5 Determinação do limite inferior de detecção para as metodologias de dos radionuclídeos ${ }^{226} \mathrm{Ra},{ }^{228} \mathrm{Ra},{ }^{210} \mathrm{~Pb}$ e ${ }^{210} \mathrm{Po}$

O limite inferior de detecção (LID) indica a sensibilidade da metodologia a baixas concentrações. O LID para todos os radionuclídeos estudados neste presente trabalho foram determinados a partir de amostras de agua superpura 
aplicado o mesmo procedimento experimental (MOREIRA, 1993, OLIVEIRA, 1993). O cálculo foi feito de acordo com a expressão (4.15)

$$
L I D=\frac{4,66 \cdot S b}{t \cdot R Q \cdot E \cdot m}
$$

Em que:

LID: limite inferior de detecção $\left(\mathrm{mBq} \mathrm{g}^{-1}\right)$

4,66: valor tabelado que corresponde a um risco pré-determinado de que existe alguma atividade na amostra, sendo que na realidade não existe e de que não existe nada na referida amostra que na realidade existe, considerando um intervalo de confiança de $95 \%$

Sb: desvio padrão da contagem da radiação de fundo (branco) do processo

t: tempo de contagem (s)

$\mathrm{RQ}$ : rendimento químico

E: eficiência do sistema de contagem

M: massa da amostra (g)

Na TAB. 4.9 são apresentados os resultados obtidos para o LID dos radionuclídeos ${ }^{226} \mathrm{Ra},{ }^{228} \mathrm{Ra},{ }^{210} \mathrm{~Pb}$ e ${ }^{210} \mathrm{Po}$ em $\mathrm{mBq} \mathrm{g}^{-1}$.

TABELA 4.9 - Resultados obtidos para o LID dos radionuclídeos ${ }^{226} \mathrm{Ra},{ }^{228} \mathrm{Ra},{ }^{210} \mathrm{~Pb}$ $\mathrm{e}^{210} \mathrm{Po}$ em $\mathrm{mBq} \mathrm{g}^{-1}$

\begin{tabular}{cc}
\hline Radionuclídeo & LID $\left(\mathbf{m B q ~ ~ ^ { - 1 } )}\right.$ \\
\hline${ }^{226} \mathbf{R a}$ & 2,2 \\
${ }^{228} \mathbf{R a}$ & 3,7 \\
\hline${ }^{210} \mathrm{~Pb}$ & 4,9 \\
${ }^{210} \mathrm{Po}$ & 4,2 \\
\hline
\end{tabular}

\subsection{Verificação das metodologias}

As metodologias para determinação da atividade de concentração dos radionuclídeos ${ }^{226} \mathrm{Ra},{ }^{228} \mathrm{Ra},{ }^{210} \mathrm{~Pb}$ e ${ }^{210} \mathrm{Po}$ foram validadas utilizando o padrão IAEA-385 (Natural and artificial radionuclides in sediment from the Irish Sea), da Internacional Atomic Energy Agency. Foram pesados 0,5 g do padrão e submetidos 
aos mesmos procedimentos radioquímicos para determinação de ${ }^{226} \mathrm{Ra},{ }^{228} \mathrm{Ra}$, ${ }^{210} \mathrm{~Pb}$ e ${ }^{210} \mathrm{Po}$. Os resultados são apresentados na TAB 4.10 juntamente com os respectivos intervalos de confiança, desvios padrão relativo (DPR) e erros relativo (ER).

TABELA 4.10 - Valores de Atividade de concentração calculada e certificada dos radionuclídeos ${ }^{226} \mathrm{Ra},{ }^{228} \mathrm{Ra},{ }^{210} \mathrm{~Pb}$ e ${ }^{210} \mathrm{Po}$ em Bq kg-1 , intervalo de confiança, desvios padrão relativo e erros relativo

\begin{tabular}{ccccc}
\hline Radionuclídeo & $\begin{array}{c}\text { Valor } \\
\text { Calculado }\end{array}$ & $\begin{array}{c}\text { Valor } \\
\text { Certificado }\end{array}$ & $\begin{array}{c}\text { DPR } \\
\%\end{array}$ & $\begin{array}{c}\text { ER } \\
\%\end{array}$ \\
\hline${ }^{226} \mathbf{R a}$ & $20,5 \pm 3,9$ & 21,9 & 19,0 & 6,8 \\
\hline${ }^{228} \mathbf{R a}$ & $35,8 \pm 4,8$ & 32,0 & 13,4 & 0,6 \\
\hline${ }^{210} \mathbf{P b}$ & $32,5 \pm 6,5$ & 32,9 & 20,0 & 1,2 \\
\hline${ }^{210} \mathbf{P o}$ & $34,9 \pm 0,8$ & 32,9 & 2,3 & 5,7 \\
\hline
\end{tabular}

\subsection{Resultados das metodologias para os derivados da Nicotiana tabacum L.}

Todos os resultados obtidos no presente trabalho por técnica analítica empregada são apresentados nos Apêndices relacionados abaixo:

A.1 Concentração dos elementos determinados em $\mathrm{mg} \mathrm{kg}^{-1}$ nos cigarros não aromatizados determinados pela técnica INAA;

A.2 Concentração dos elementos determinados em $\mathrm{mg} \mathrm{kg}^{-1}$ nos cigarros aromatizados determinados pela técnica INAA;

A.3 Concentração dos elementos determinados em $\mathrm{mg} \mathrm{kg}^{-1}$ nos cigarros de palha determinados pela técnica INAA;

A.4 Concentração dos elementos determinados em $\mathrm{mg} \mathrm{kg}^{-1}$ nos charutos determinados pela técnica INAA;

A.5 Concentração dos elementos determinados em $\mathrm{mg} \mathrm{kg}^{-1}$ nos fumos de corda e rapé determinados pela técnica INAA;

B.1 Concentração dos elementos determinados em $\mathrm{mg} \mathrm{kg}^{-1}$ nos cigarros não aromatizados determinados pela técnica EDXRF; 
B.2 Concentração dos elementos determinados em $\mathrm{mg} \mathrm{kg}^{-1}$ nos cigarros aromatizados determinados pela técnica EDXRF;

B.3 Concentração dos elementos determinados em $\mathrm{mg} \mathrm{kg}^{-1}$ nos cigarros de palha determinados pela técnica EDXRF;

B.4 Concentração dos elementos determinados em $\mathrm{mg} \mathrm{kg}^{-1}$ nos charutos determinados pela técnica EDXRF;

B.5 Concentração dos elementos determinados em $\mathrm{mg} \mathrm{kg}^{-1}$ nos fumos de corda e rapés determinados pela técnica EDXRF;

C.1 Concentração dos radionuclídeos estudados em $\mathrm{mBq}^{-1}$ nos cigarros não aromatizados;

C.2 Concentração dos radionuclídeos estudados em $\mathrm{mBq} \mathrm{g}^{-1}$ nos cigarros aromatizados;

C.3 Concentração dos radionuclídeos estudados em $\mathrm{mBq} \mathrm{g}^{-1}$ nos cigarros de palha;

C.4 Concentração dos radionuclídeos estudados em $\mathrm{mBq} \mathrm{g}^{-1}$ nos charutos;

C.5 Concentração dos radionuclídeos estudados em $\mathrm{mBq} \mathrm{g}^{-1}$ nos fumos de corda e rapés.

\subsubsection{Análise de Ativação com Nêutrons Instrumental - INAA}

Para melhor visualização dos resultados os elementos determinados foram agrupados em elementos maiores: $\mathrm{Ca}, \mathrm{K}, \mathrm{Fe}$ e $\mathrm{Na}$; elementos traço: $\mathrm{As}, \mathrm{Ba}$, $\mathrm{Br}, \mathrm{Co}, \mathrm{Cr}, \mathrm{Cs}, \mathrm{Hf}, \mathrm{Rb}, \mathrm{Sb}, \mathrm{Se}, \mathrm{Ta}, \mathrm{Th}, \mathrm{U}$ e Zn; e elementos terras raras: Ce, Eu, La, $\mathrm{Lu}, \mathrm{Nd}, \mathrm{Sc}, \mathrm{Sm}, \mathrm{Tb}$ e $\mathrm{Yb}$.

\subsubsection{Elementos maiores $\mathrm{Ca}, \mathrm{K}$, Fe, e $\mathrm{Na}$}

Na TAB. 4.11; são apresentados os valores de concentrações médios, mínimos e máximos dos elementos maiores $\mathrm{Ca}$, Fe, $\mathrm{K}$ e Na em \% nas amostras de cigarros não aromatizados, cigarros aromatizados, cigarros de palha, charutos, fumos de corda e rapé, respectivamente. 
Pode se observar que o elemento $\mathrm{Ca}$ apresentou os maiores valores de concentração na maioria das amostras e os menores valores foram obtidos para o elemento $\mathrm{Na}$.

Os valores do elemento Ca obtidos nas amostras estão de acordo com os obtidos por LEFFINGWELL (1999) que determinou o elemento Ca, \%, em folhas de tabaco e obteve valores que variaram de 1,59\% a 5,72\%. A maior concentração foi obtida do elemento Ca no cigarro de palha CPPF, 6,6\% e a menor concentração no fumo de corda aromatizado de morango FCAMO 1,1\% (Apêndice A).

Na FIG.4.7 pode-se observar as variações da concentração do elemento Ca (\%) dentro dos grupos analisados de amostras analisadas e verificar o número de amostras de cada grupo. Na figura observa-se que o grupo que apresentou concentrações mais homogêneas de $\mathrm{Ca}$ foi o de cigarros não aromatizados e o grupo de apresentou maior variação de concentração foi o de cigarros de palha. 
TABELA 4.11 - Valores médios, mínimos e máximos de concentração dos elementos Ca, Fe, K e Na (\%) nos derivados da Nicotiana tabacum L., pela técnica de INAA

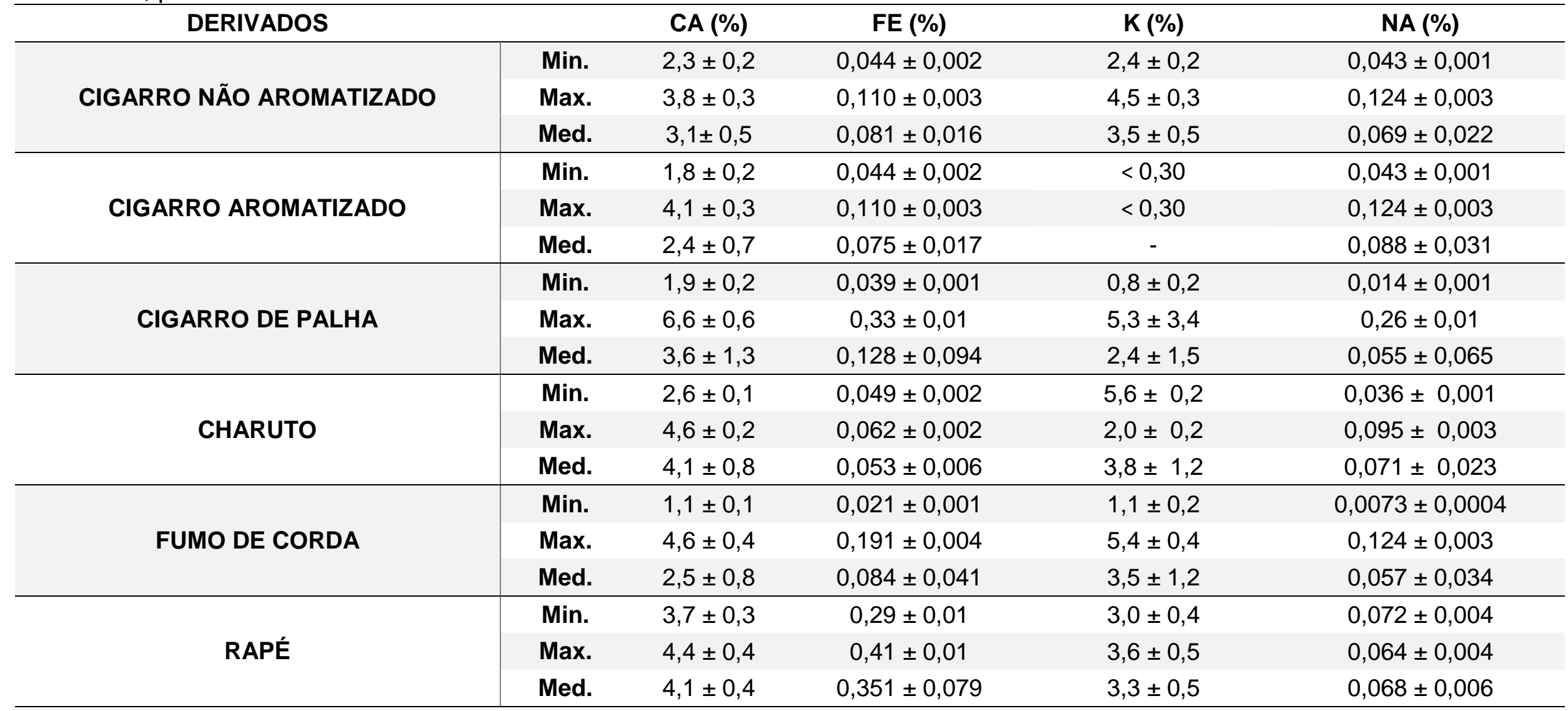


Isso pode ser explicado pelo fato de que quase todo o tabaco utilizado nos cigarros industrializados (cigarros não aromatizados e cigarros aromatizados) ser cultivado em grande escala na região sul do país (Estados de Santa Catarina, Paraná e Rio Grande do Sul) (SINDITABACO, 2017) enquanto que outros derivados de tabaco como os cigarros de palha e os fumo de corda são produzidos em pequena escala em unidades rurais de todo o território brasileiro; as amostras de cigarros de palha e fumo de corda de este trabalho foram adquiridas em diversas cidades e Estados brasileiros, sendo a maioria de origem de Minas Gerais.

FIGURA 4.7 - Variação da concentração do elemento Ca (\%) nos derivados da Nicotiana tabacum L. pela técnica de INAA.

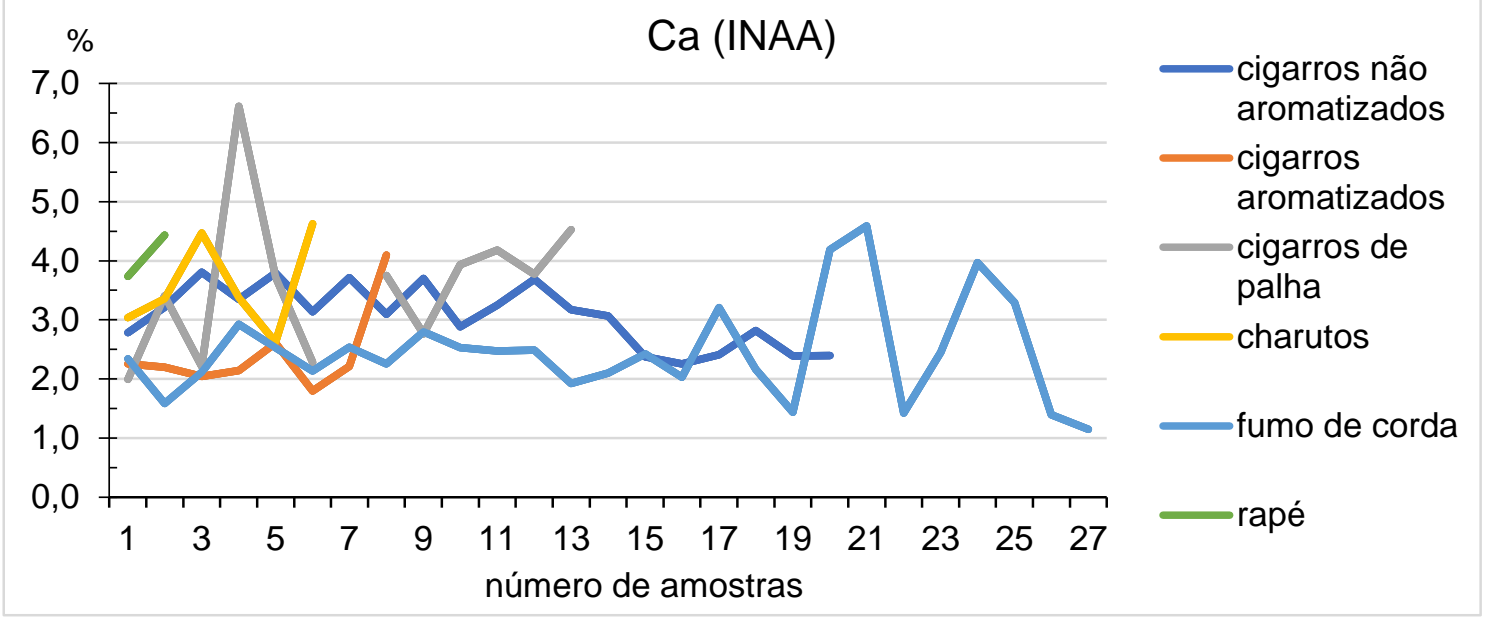

A maioria das amostras de cigarros aromatizados apresentaram valores de concentração do elemento $K$ inferiores ao LID de $<0,30$, com exceção da amostra CAMBI (Apêndice A.1) que apresentou uma concentração de 3,3 \%, semelhante às encontradas nos cigarros não aromatizados; uma explicação possível seria que este produto possui uma cápsula aromatizada na parte do filtro e como instrução da embalagem é necessária a quebra dessa cápsula para liberação do aroma. Os valores do elemento K obtidos nas amostras estão de acordo com os obtidos por LEFFINGWELL (1999) que obteve valores que variaram de $1,65 \%$ a $3,7 \%$ em folhas de tabaco.

Na FIG 4.8 observa-se a diferença de concentração dos cigarros aromatizados e os demais derivados estudados, e assim como para o elemento $\mathrm{Ca}$ o grupo de amostras que apresentou menor variação na concentração de $\mathrm{K}$ foram 
os de cigarros não aromatizados e o que apresentou maior variação foram os de cigarros de palha.

FIGURA 4.8 - Variação da concentração do elemento K (\%) nos derivados da Nicotiana tabacum $\mathrm{L}$. pela técnica de INAA.

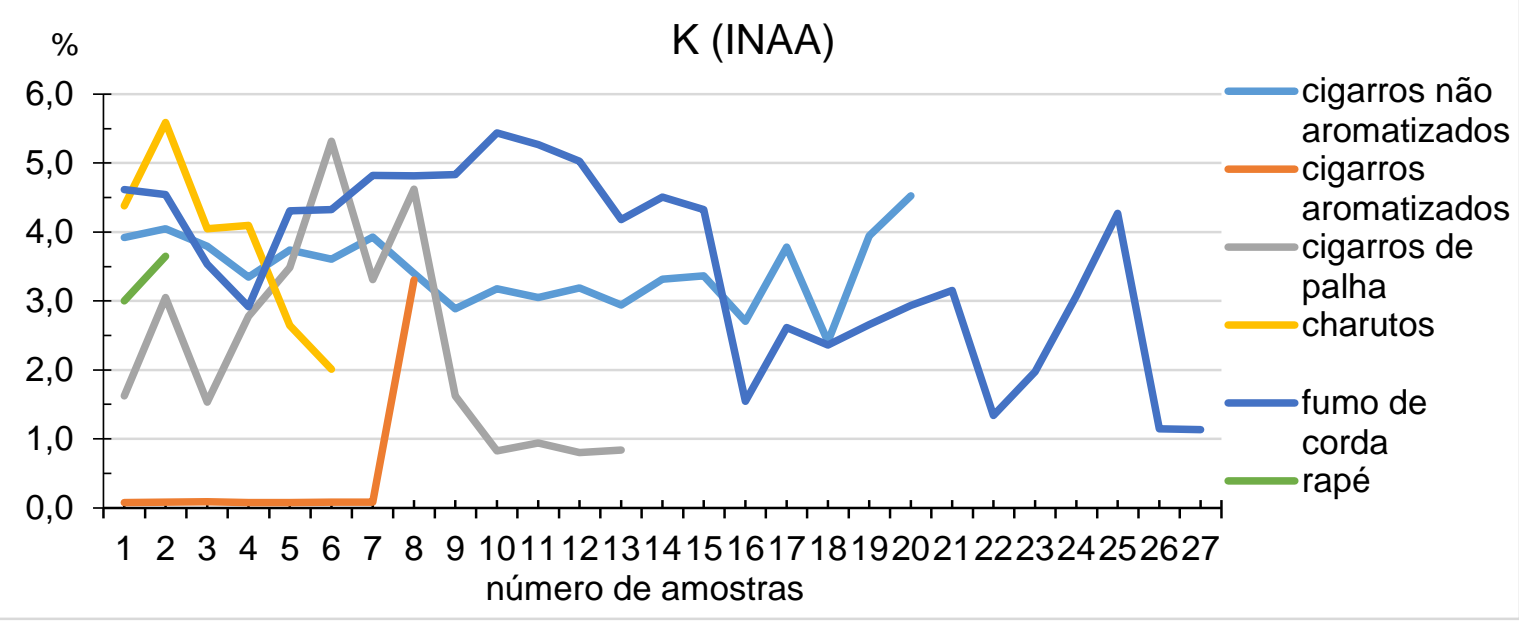

Os elementos $\mathrm{Fe}$ e $\mathrm{Na}$ apresentaram os menores valores de concentração em todas as amostras, quando comparados com os outros elementos maiores determinados.

Na FIG.4.9 e 4.10 são apresentadas as variações das concentrações dos elementos Fe (\%) e $\mathrm{Na}(\%)$ para todo o grupo amostral, respectivamente. A concentração média do elemento Fe nas amostras de cigarros não aromatizados e de fumo de corda foi de $0,08 \%$, nos cigarros aromatizados 0,07\%, nos charutos 0,05\%, nos cigarros de palha 0,13\% e a maior concentração média foi determinada nas amostras de rapé com valor de 0,35\%. A concentração média do elemento $\mathrm{Na}$ foi de $0,07 \%$ nas amostras de cigarros não aromatizados, charutos e rapé; $0,06 \%$ nas de cigarros de palha e fumos de corda e de 0,09\% nas de cigarros aromatizados. 
FIGURA 4.9 - Variação da concentração do elemento Fe (\%) nos derivados da Nicotiana tabacum $\mathrm{L}$. pela técnica de INAA.

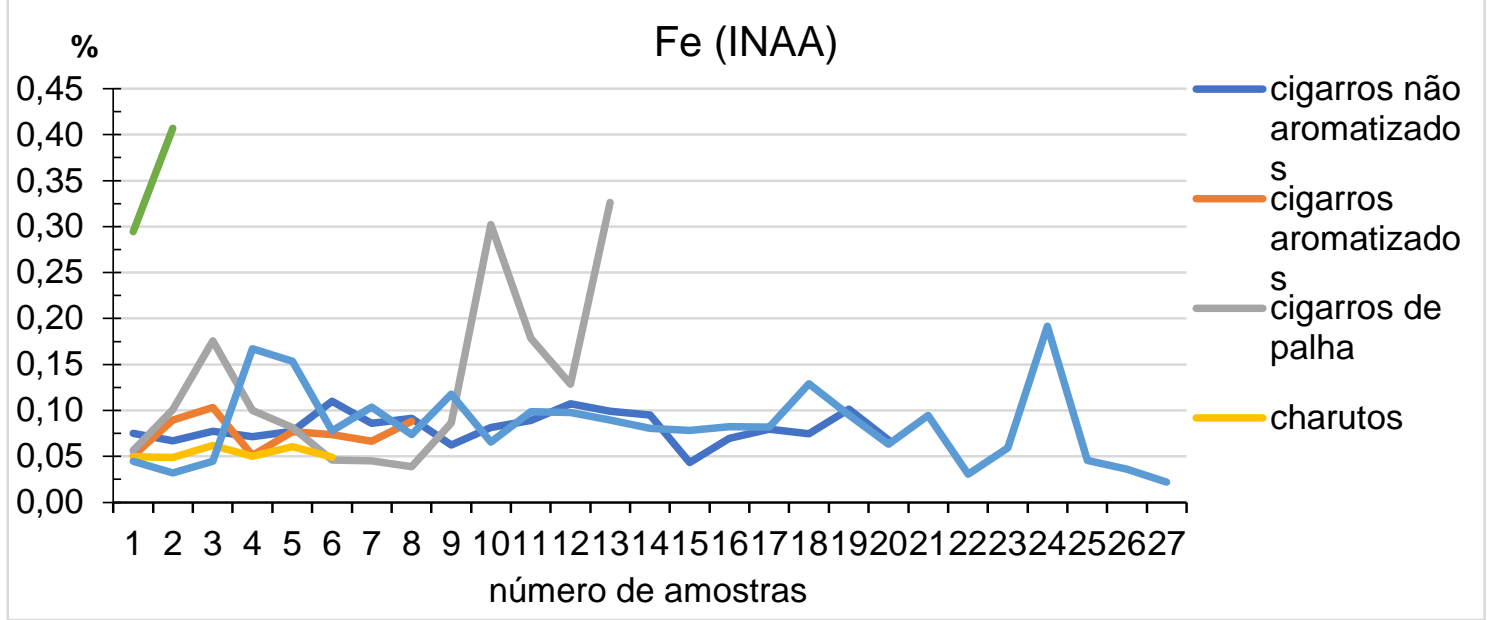

FIGURA 4.10 - Variação da concentração do elemento $\mathrm{Na}$ (\%) nos derivados da Nicotiana tabacum $\mathrm{L}$. pela técnica de INAA.

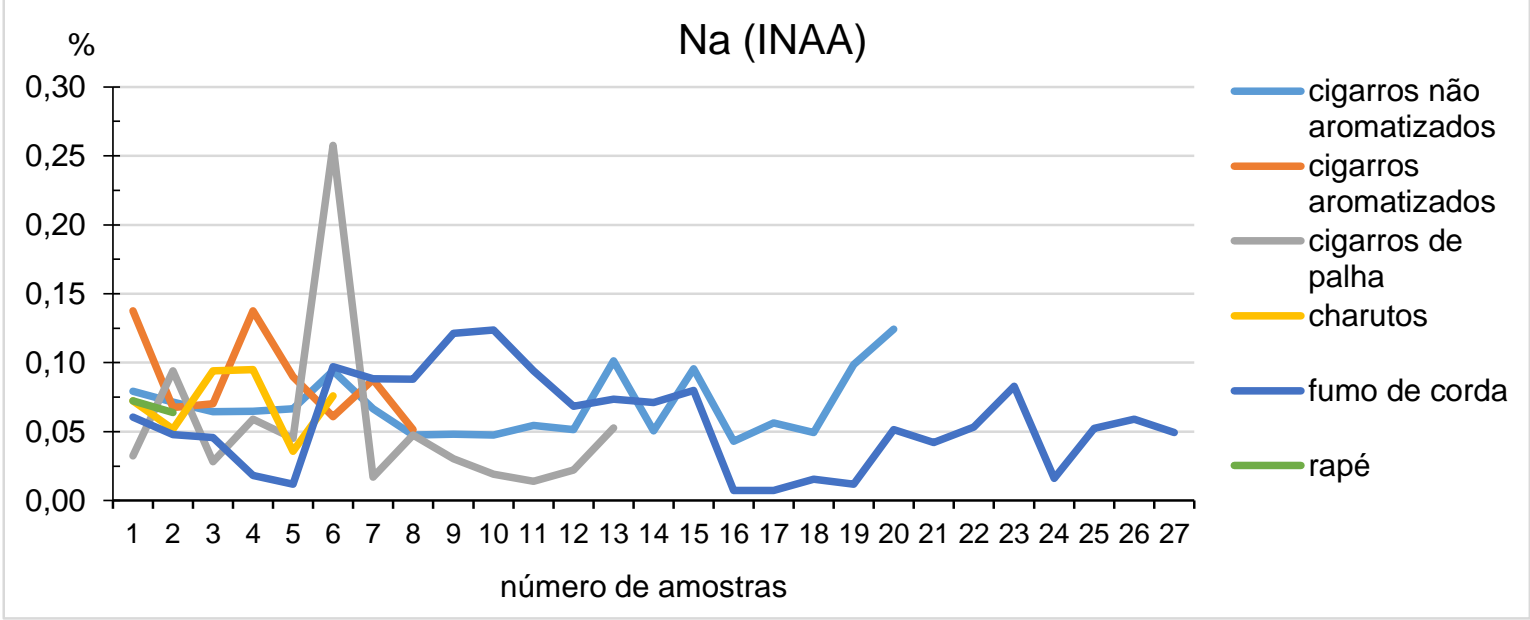

\subsubsection{Elementos Traço As, Ba, Br, Co, Cr, Cs, Hf, Rb, Sb, Se, Ta, Th, U e Zn}

Na TAB. 4.12 são apresentados os resultados das concentrações dos elementos traço, As, Ba, Br, Co, Cr, Cs, Hf, Rb e Zn em mg kg-1 nas amostras de cigarros não aromatizados, cigarros aromatizados, cigarros de palha, charutos, fumos de corda e rapé. 
TABELA 4.12 - Valores médios, mínimos e máximos de concentração dos elementos traço As, Ba, Br, Co, Cr, Cs, Hf, Rb e Zn ( $\left.\mathrm{mg} \mathrm{kg}^{-1}\right)$ nos derivados da Nicotiana tabacum L., pela técnica de INAA

\begin{tabular}{|c|c|c|c|c|c|c|c|c|c|c|}
\hline DERIVADOS & & As & $\mathrm{Ba}$ & $\mathrm{Br}$ & Co & $\mathrm{Cr}$ & Cs & Hf & $\mathbf{R b}$ & $\mathrm{Zn}$ \\
\hline \multirow{3}{*}{$\begin{array}{l}\text { CIGARROS NÃO } \\
\text { AROMATIZADOS }\end{array}$} & Min. & $1,4 \pm 0,1$ & $89 \pm 7$ & $6,9 \pm 0,03$ & $0,64 \pm 0,03$ & $1,1 \pm 0,1$ & $0,11 \pm 0,02$ & $0,11 \pm 0,02$ & $22 \pm 1$ & $26 \pm 1$ \\
\hline & Max. & $2,9 \pm 0,2$ & $207 \pm 25$ & $87 \pm 1$ & $1,08 \pm 0,04$ & $4,4 \pm 0,3$ & $0,53 \pm 0,05$ & $0,32 \pm 0,03$ & $43 \pm 2$ & $45 \pm 2$ \\
\hline & Med. & $2,04 \pm 0,65$ & $140 \pm 32$ & $56 \pm 23$ & $0,89 \pm 0,11$ & $2,0 \pm 0,8$ & $0,18 \pm 0,09$ & $0,21 \pm 0,05$ & $28 \pm 5$ & $38 \pm 5$ \\
\hline \multirow{3}{*}{$\begin{array}{c}\text { CIGARROS } \\
\text { AROMATIZADOS }\end{array}$} & Min. & $2,1 \pm 0,3$ & $82 \pm 11$ & $21,2 \pm 0,3$ & $0,52 \pm 0,03$ & $1,4 \pm 0,2$ & $0,13 \pm 0,03$ & $0,09 \pm 0,02$ & $21 \pm 2$ & $31 \pm 2$ \\
\hline & Max. & $2,9 \pm 0,4$ & $165 \pm 19$ & $72 \pm 1$ & $1,07 \pm 0,05$ & $2,6 \pm 0,3$ & $0,40 \pm 0,10$ & $0,36 \pm 0,04$ & $33 \pm 3$ & $43 \pm 3$ \\
\hline & Med. & $2,5 \pm 0,5$ & $121 \pm 30$ & $49 \pm 17$ & $0,78 \pm 0,19$ & $2,0 \pm 0,2$ & $0,22 \pm 0,09$ & $0,22 \pm 0,08$ & $28 \pm 3$ & $38 \pm 4$ \\
\hline \multirow{3}{*}{$\begin{array}{c}\text { CIGARROS DE } \\
\text { PALHA }\end{array}$} & Min. & $1,9 \pm 0,2$ & $67 \pm 9$ & $29,7 \pm 0,2$ & $0,44 \pm 0,02$ & $0,8 \pm 0,1$ & $0,13 \pm 0,02$ & $0,14 \pm 0,02$ & $14 \pm 1$ & $43 \pm 2$ \\
\hline & Max. & $6,4 \pm 0,4$ & $197 \pm 15$ & $153 \pm 1$ & $1,8 \pm 0,1$ & $8 \pm 1$ & $0,50 \pm 0,10$ & $1,2 \pm 0,1$ & $51 \pm 3$ & $137 \pm 7$ \\
\hline & Med. & $3,1 \pm 1,9$ & $112 \pm 38$ & $55 \pm 32$ & $0,84 \pm 0,44$ & $3,0 \pm 2,2$ & $0,29 \pm 0,10$ & $0,33 \pm 0,27$ & $27 \pm 14$ & $64 \pm 25$ \\
\hline \multirow{3}{*}{ CHARUTO } & Min. & $2,4 \pm 0,2$ & $74 \pm 4$ & $48,8 \pm 0,4$ & $0,29 \pm 0,02$ & $1,1 \pm 0,2$ & $0,09 \pm 0,03$ & $0,27 \pm 0,03$ & $19 \pm 1$ & $36 \pm 1$ \\
\hline & Max. & $2,5 \pm 0,2$ & $165 \pm 21$ & $164 \pm 1$ & $0,58 \pm 0,03$ & $4,7 \pm 0,4$ & $0,2 \pm 0,04$ & $0,52 \pm 0,04$ & $43 \pm 3$ & $80 \pm 3$ \\
\hline & Med. & $2,44 \pm 0,02$ & $102 \pm 36$ & $111 \pm 46$ & $0,42 \pm 0,10$ & $2,3 \pm 1,4$ & $0,14 \pm 0,04$ & $0,37 \pm 0,08$ & $28 \pm 8$ & $68 \pm 16$ \\
\hline \multirow{3}{*}{$\begin{array}{l}\text { FUMOS DE } \\
\text { CORDA }\end{array}$} & Min. & $1,5 \pm 0,2$ & $41 \pm 7$ & $21,7 \pm 0,2$ & $0,15 \pm 0,01$ & $1,1 \pm 0,1$ & $0,09 \pm 0,01$ & $0,08 \pm 0,01$ & $12 \pm 1$ & $22 \pm 1$ \\
\hline & Max. & $3,2 \pm 0,2$ & $207 \pm 14$ & $226 \pm 1$ & $1,4 \pm 0,1$ & $5 \pm 1$ & $0,45 \pm 0,04$ & $0,6 \pm 0,1$ & $46 \pm 3$ & $127 \pm 4$ \\
\hline & Med. & $2,0 \pm 0,5$ & $123 \pm 46$ & $67 \pm 45$ & $0,81 \pm 0,37$ & $2,1 \pm 1,0$ & $0,20 \pm 0,09$ & $0,24 \pm 0,11$ & $25 \pm 8$ & $74 \pm 33$ \\
\hline \multirow{3}{*}{ RAPÉ } & Min. & $<1,4$ & $94 \pm 16$ & $61,1 \pm 0,3$ & $0,80 \pm 0,04$ & $0,25 \pm 0,02$ & $0,25 \pm 0,02$ & $1,8 \pm 0,1$ & $33 \pm 3$ & $84 \pm 4$ \\
\hline & Max. & $<1,4$ & $139 \pm 23$ & $72,9 \pm 0,3$ & $1,30 \pm 0,06$ & $0,5 \pm 0,1$ & $0,5 \pm 0,1$ & $1,9 \pm 0,1$ & $40 \pm 2$ & $75 \pm 3$ \\
\hline & Med. & $<1,4$ & $116 \pm 32$ & $67,0 \pm 8,3$ & $1,05 \pm 0,03$ & $0,4 \pm 0,2$ & $0,38 \pm 0,18$ & $1,87 \pm 0,03$ & $36 \pm 5$ & $79 \pm 6$ \\
\hline
\end{tabular}


Os elementos Sb, Ta e U apresentaram valores de concentração abaixo do LID em todas as amostras.

Apenas dois grupos de amostras apresentaram concentrações detectáveis para o elemento Se: quatro amostras de fumo de corda (FCP, FCA4, FCA5 e FCDM) e 1 amostra de rapé (RPCM), TAB 4.13.

TABELA 4.13 - Concentração do elemento Se $\left(\mathrm{mg} \mathrm{kg}^{-1}\right)$ nas amostras de fumo de corda e rapé pela técnica de INAA.

\section{Fumo de corda}

Rapé

\begin{tabular}{|c|c|c|}
\hline FCP & $0,4 \pm 0,1$ & RPCM $\quad 1,8 \pm 1,2$ \\
\hline FCA4 & $0,7 \pm 0,4$ & \\
\hline FCA5 & $0,4 \pm 0,2$ & \\
\hline FCA6 & $0,3 \pm 0,2$ & \\
\hline FCDM & $0,6 \pm 0,5$ & \\
\hline lédia \pm DP & $0,5 \pm 0,2$ & \\
\hline
\end{tabular}

O elemento Th também apresentou concentração detectável apenas em 14 amostras: sete amostras de fumo de corda, 5 amostras de cigarros de palha e nas duas amostras de rapé (LID =0,38), TAB 4.14.

TABELA 4.14 - Concentração do elemento Th $\left(\mathrm{mg} \mathrm{kg}^{-1}\right)$ nas amostras de cigarro de palha, fumo de corda e rapé pela técnica de INAA

\begin{tabular}{ll|ll|ll}
\multicolumn{2}{c|}{ Cigarro de palha } & \multicolumn{2}{c|}{ Fumo de Corda } & \multicolumn{2}{c}{ Rapé } \\
\hline CPP & $\mathbf{0 , 3 8} \pm \mathbf{0 , 0 3}$ & FCP & $\mathbf{0 , 4 8} \pm \mathbf{0 , 0 4}$ & RPAR & $1,0 \pm 0,2$ \\
\hline CPCR & $0,82 \pm 0,05$ & FCPF & $0,55 \pm 0,04$ & RPCM & $1,2 \pm 0,2$ \\
\hline CPPG & $0,75 \pm 0,05$ & FCA4 & $0,6 \pm 0,1$ & Média \pm DP & $1,1 \pm 0,1$ \\
\hline CPSP & $0,5 \pm 0,04$ & FCA5 & $0,4 \pm 0,1$ & & \\
\hline CPVC & $1,6 \pm 0,1$ & FCDD & $0,4 \pm 0,1$ & & \\
\hline Média \pm DP & $0,80 \pm 0,46$ & FCDM & $0,4 \pm 0,1$ & & \\
\hline & & FCDMP & $0,9 \pm 0,1$ & & \\
\hline & & Média \pm DP & $0,6 \pm 0,2$ & & \\
\hline
\end{tabular}


O elemento As foi determinado em apenas algumas amostras (FIG. 4.11); a concentração variou de 1,41 $\pm 0,10 \mathrm{mg} \mathrm{kg}^{-1}$ na amostra de cigarro não aromatizado CCMG a 6,4 $\mathrm{mg} \mathrm{kg}^{-1}$ no cigarro de palha CPVC (Apêndice A). FRANCISCONI et al. (2011) analisando diversas marcas de cigarros brasileiros encontrou valores médios de concentração do elemento As de 0,25 $\pm 0,1 \mathrm{mg} \mathrm{kg}^{-1}$, valor muito inferior aos valores médios determinados no presente trabalho para os cigarros não aromatizados e cigarros aromatizados, 2,04 \pm 0,65 $\mathrm{mg} \mathrm{kg}^{-1}$ e 2,5 \pm 0,5 $\mathrm{mg} \mathrm{kg}^{-1}$, respectivamente.

FIGURA 4.11 - Variação da concentração do elemento As $\left(\mathrm{mg} \mathrm{kg}^{-1}\right)$ nos derivados da Nicotiana tabacum L. pela técnica de INAA.

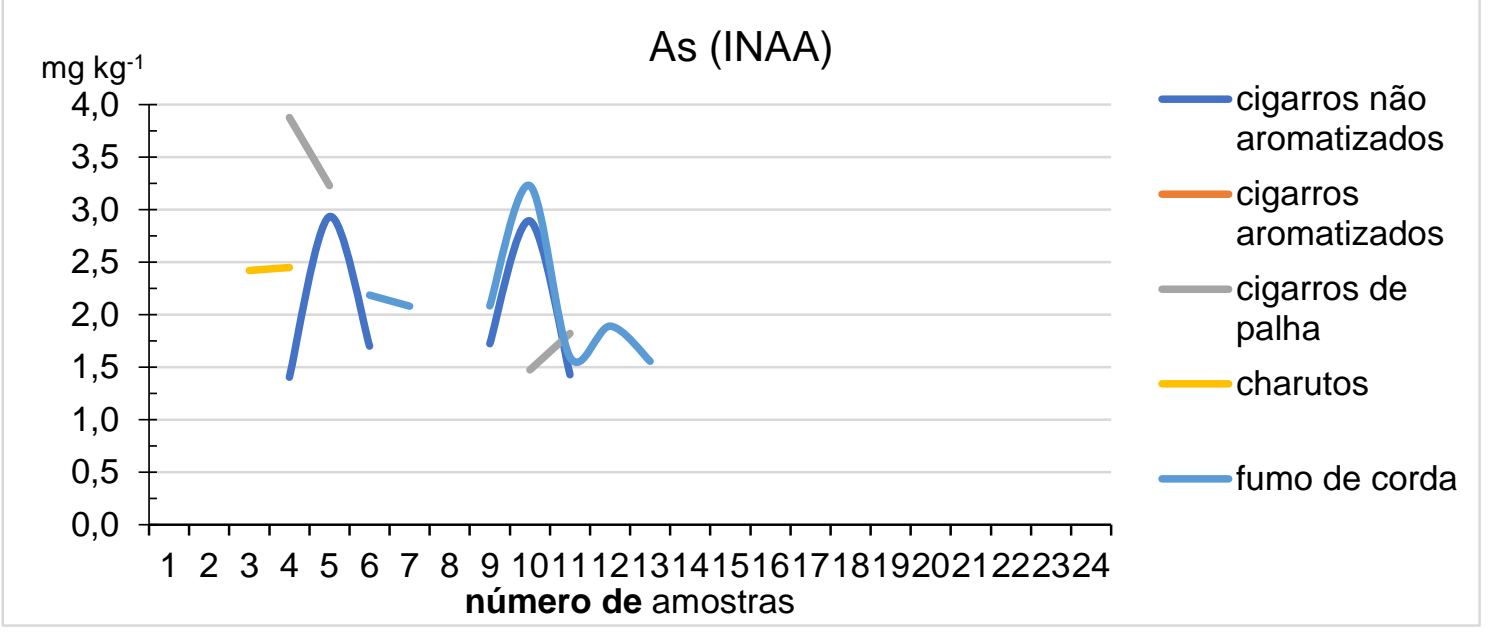

Nas FIG 4.12 a 4.20 são apresentadas as concentrações dos elementos $\mathrm{Ba}, \mathrm{Br}, \mathrm{Co}, \mathrm{Cr}, \mathrm{Cs}, \mathrm{Hf}, \mathrm{Rb}, \mathrm{Zn}$ e Th nas amostras de derivados da Nicotina tabacum I.

As concentrações do elemento Ba variaram do LID (39 $\mathrm{mg} \mathrm{kg}^{-1}$ ) na amostra de charuto CHMPC a $207 \pm 14 \mathrm{mg} \mathrm{kg}^{-1}$ na amostra de fumo de corda FCDM (Apêndice A). Pode-se observar na FIG. 4.7 que o grupo de amostras que apresentou maior variação nas concentrações do elemento Ba foi o de fumo de corda. 
FIGURA 4.12 - Variação da concentração do elemento Ba $\left(\mathrm{mg} \mathrm{kg}^{-1}\right)$ nos derivados da Nicotiana tabacum L. pela técnica INAA.

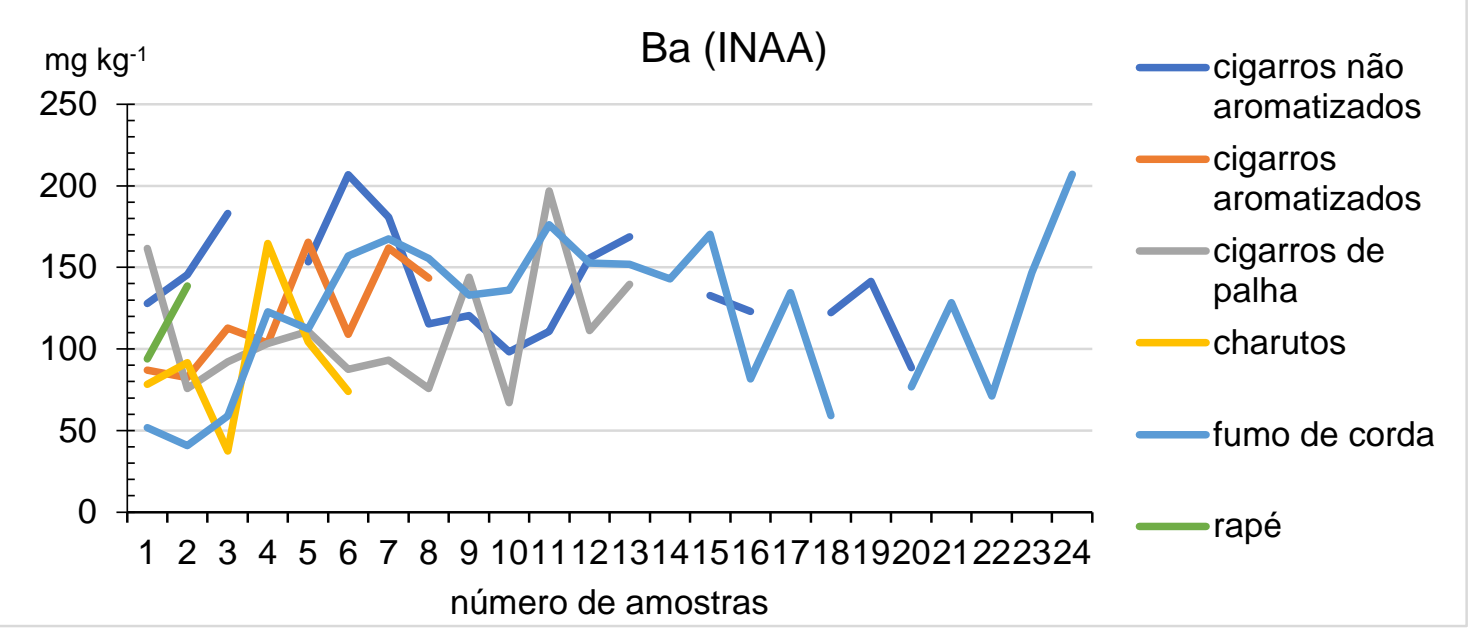

O elemento $\mathrm{Br}$ apresentou menor concentração média nos cigarros aromatizados, $49 \pm 16 \mathrm{mg} \mathrm{kg}^{-1}$ e a maior concentração média nos charutos de $111 \pm 46 \mathrm{mg} \mathrm{kg}^{-1}$, entretanto a maior concentração foi encontrada na amostra de fumo de corda $\mathrm{FCl}, 226 \pm 1 \mathrm{mg} \mathrm{kg}^{-1}$ (Apêndice A).

FIGURA 4.13 - Variação da concentração do elemento $\mathrm{Br}\left(\mathrm{mg} \mathrm{kg}^{-1}\right)$ nos derivados da Nicotiana tabacum L. pela técnica INAA.

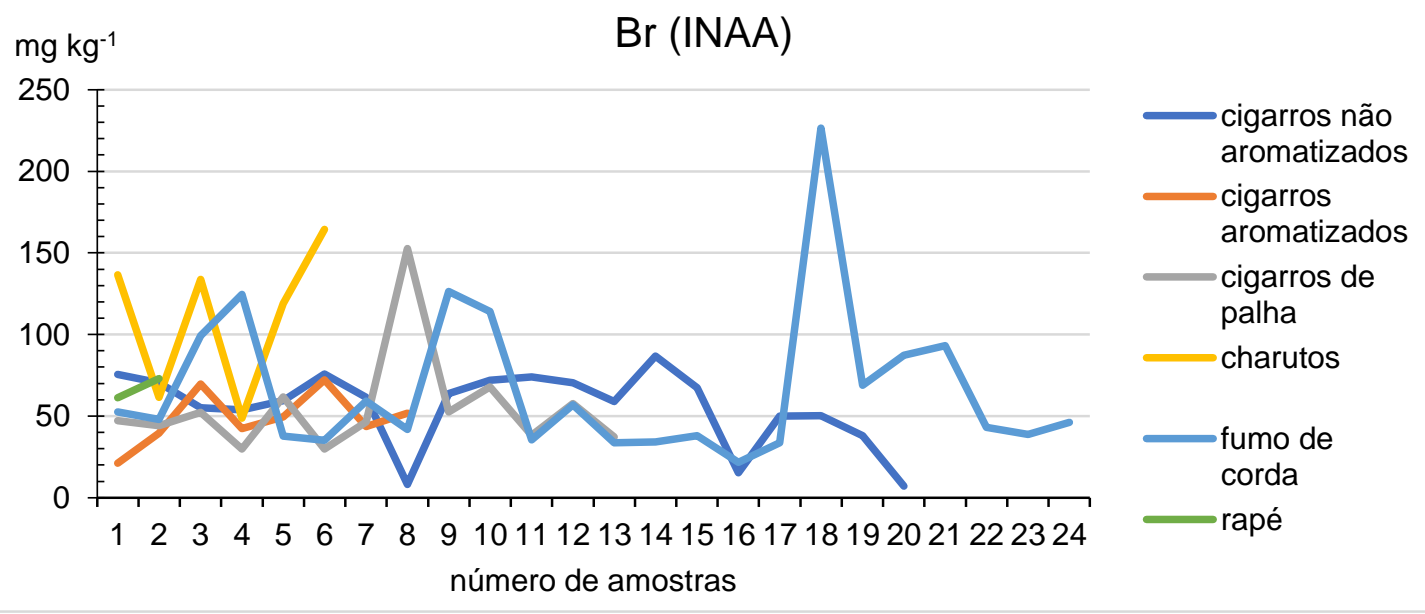

A concentração média do elemento Co variou de 0,15 $\pm 0,01 \mathrm{mg} \mathrm{kg}^{-1} \mathrm{na}$ amostra de fumo de corda FCSC a 1,8 $\pm 0,1 \mathrm{mg} \mathrm{kg}^{-1}$ na amostra de cigarro de palha CPTV. 
FIGURA 4.14 - Variação da concentração do elemento Co $\left(\mathrm{mg} \mathrm{kg}^{-1}\right)$ nos derivados da Nicotiana tabacum L. pela técnica INAA.

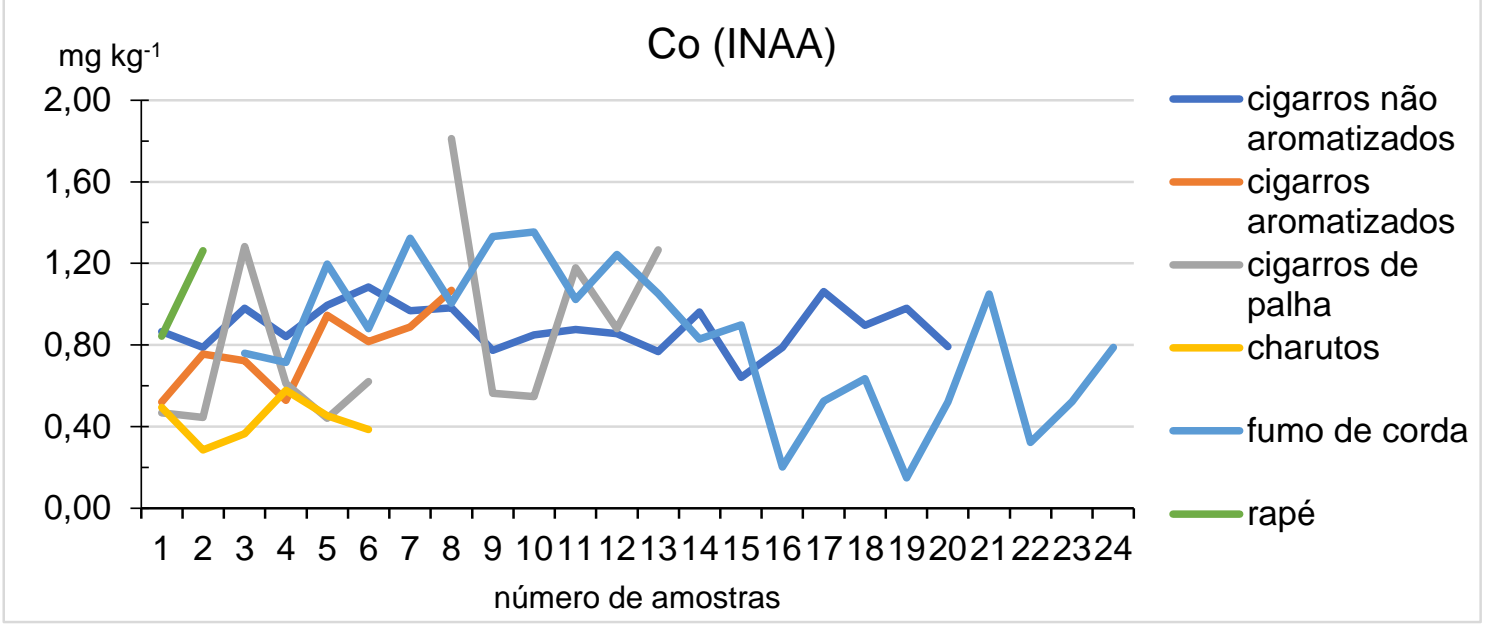

As concentrações médias do elemento Cr variaram de 0,83 \pm 0,12 mg kg${ }^{1}$ na amostra de cigarro de palha CPCPC a 7,5 $\pm 0,9 \mathrm{mg} \mathrm{kg}^{-1}$ na amostra de cigarro de palha CPVC (Apêndice A), valores muito inferiores aos determinados por KABATA-PENDIAS (2010), que variaram de $18 \mathrm{mg} \mathrm{kg}^{-1}$ a $24 \mathrm{mg} \mathrm{kg}^{-1}$ nas partes superiores da planta de tabaco.

FIGURA 4.15 - Variação da concentração do elemento $\mathrm{Cr}\left(\mathrm{mg} \mathrm{kg}^{-1}\right)$ nos derivados da Nicotiana tabacum L. pela técnica INAA.

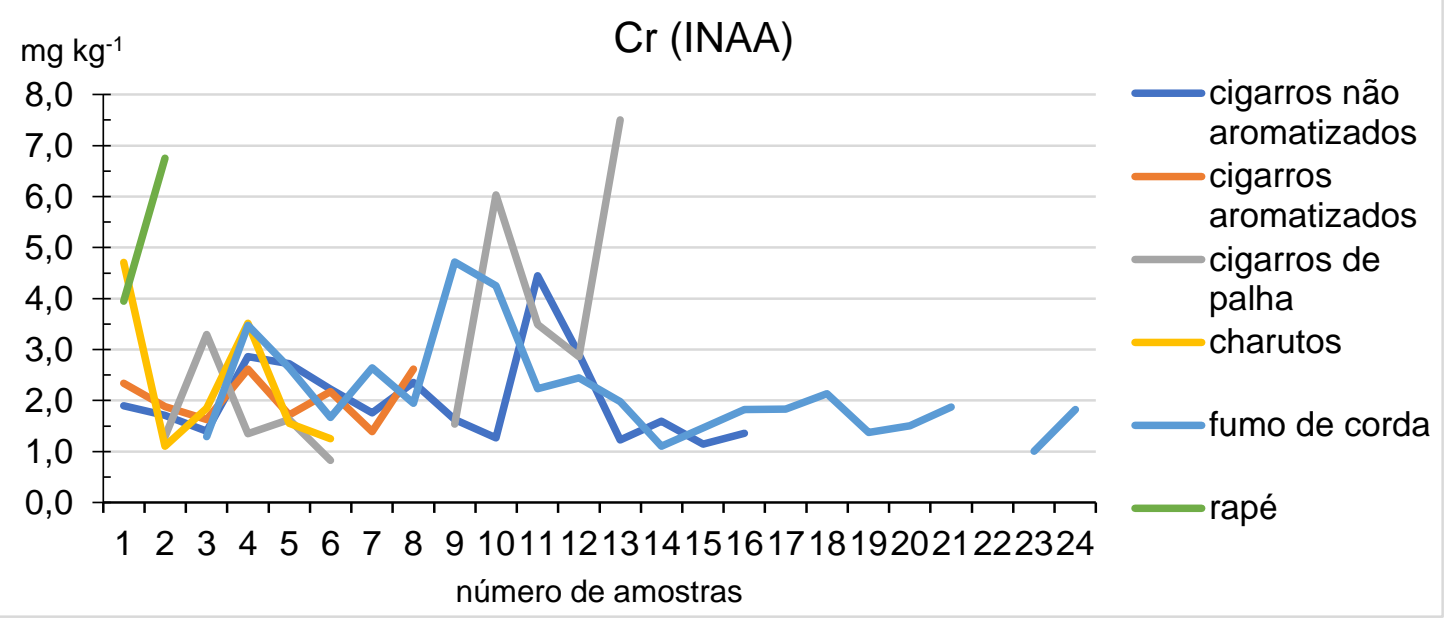

As concentrações médias de elemento Cs variaram de $0,085 \pm 0,11 \mathrm{mg} \mathrm{kg}^{-1}$ na amostra de fumo de corda FCACE a 0,53 \pm 0,05 $\mathrm{mg} \mathrm{kg}^{-1}$ na amostra de cigarro não aromatizado CCSPS (Apêndice A). Na figura 4.7 verificase que o grupo que apresentou maior variação nas concentrações do elemento Cs foi o de charuto. 
FIGURA 4.16 - Variação da concentração do elemento Cs $\left(\mathrm{mg} \mathrm{kg}^{-1}\right)$ nos derivados da Nicotiana tabacum L. pela técnica INAA.

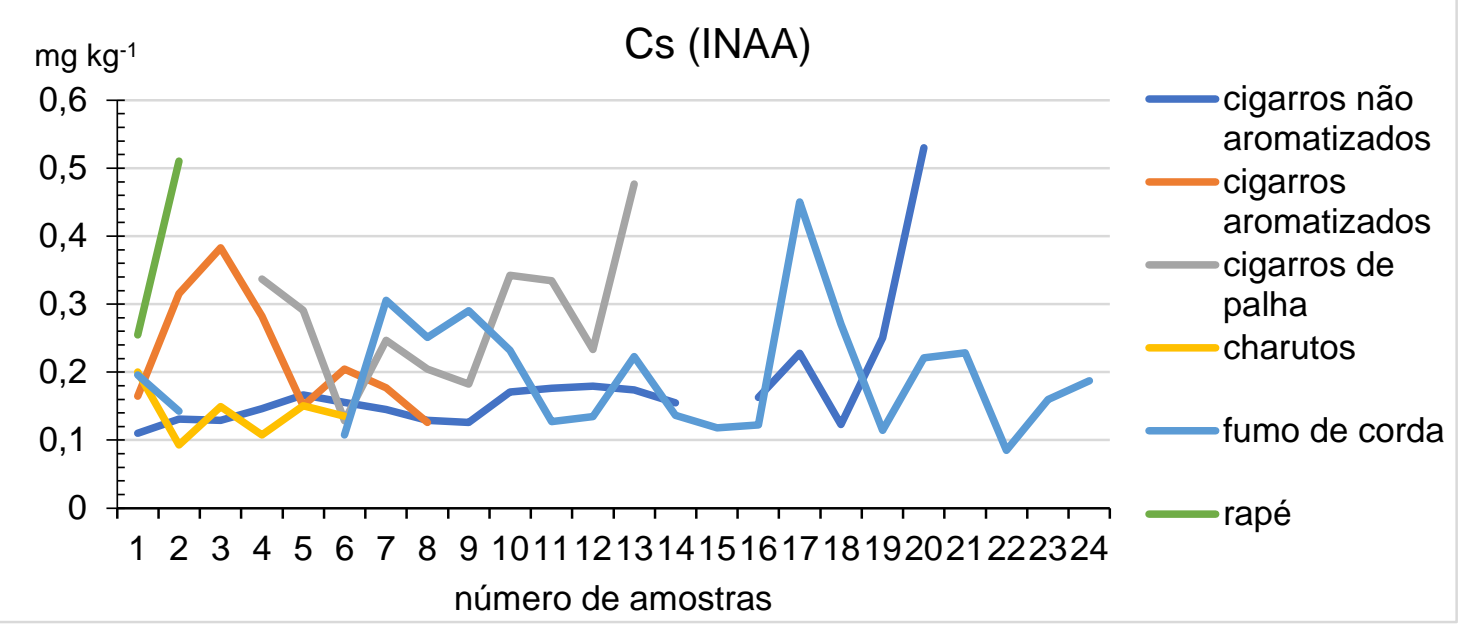

As amostras de rapé apresentaram concentrações de Hf muito maiores que as encontradas nos outros grupos: RPAR de 1,8 $\pm 0,1 \mathrm{mg} \mathrm{kg}^{-1}$ e RPCM 1,9 \pm 0,1 mg kg-1, enquanto que a maior concentração dos outros grupo foi na amostra de cigarro de palha CPVC de 1,2 $\pm 0,1 \mathrm{mg} \mathrm{kg}^{-1}$. A menor concentração determinada par o elemento $\mathrm{Hf}$ foi determinada na amostra de fumo de corda aromatizado FCACE, com valor de 0,10 $\pm 0,01 \mathrm{mg} \mathrm{kg}^{-1}$ (Apêndice A.5).

FIGURA 4.17 - Variação da concentração do elemento Hf $\left(\mathrm{mg} \mathrm{kg}^{-1}\right)$ nos derivados da Nicotiana tabacum L. pela técnica INAA.

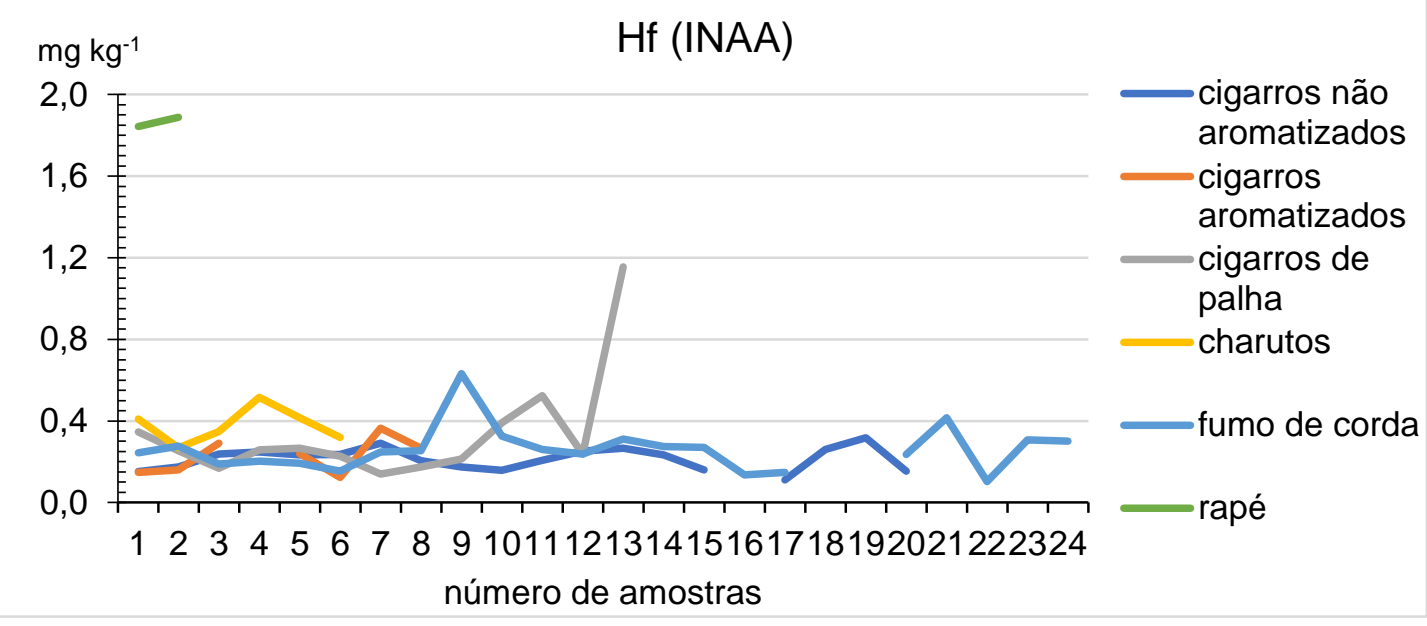

O elemento $\mathrm{Rb}$ apresentou concentrações que variaram de $12 \pm 1 \mathrm{mg} \mathrm{kg}$ ${ }^{1}$ na amostra de fumo de corda FCAAL a $51 \pm 3 \mathrm{mg} \mathrm{kg}^{-1}$ na amostra de cigarro de palha CPRN. 
FIGURA 4.18 - Variação da concentração do elemento Rb $\left(\mathrm{mg} \mathrm{kg}^{-1}\right)$ nos derivados da Nicotiana tabacum L. pela técnica INAA.

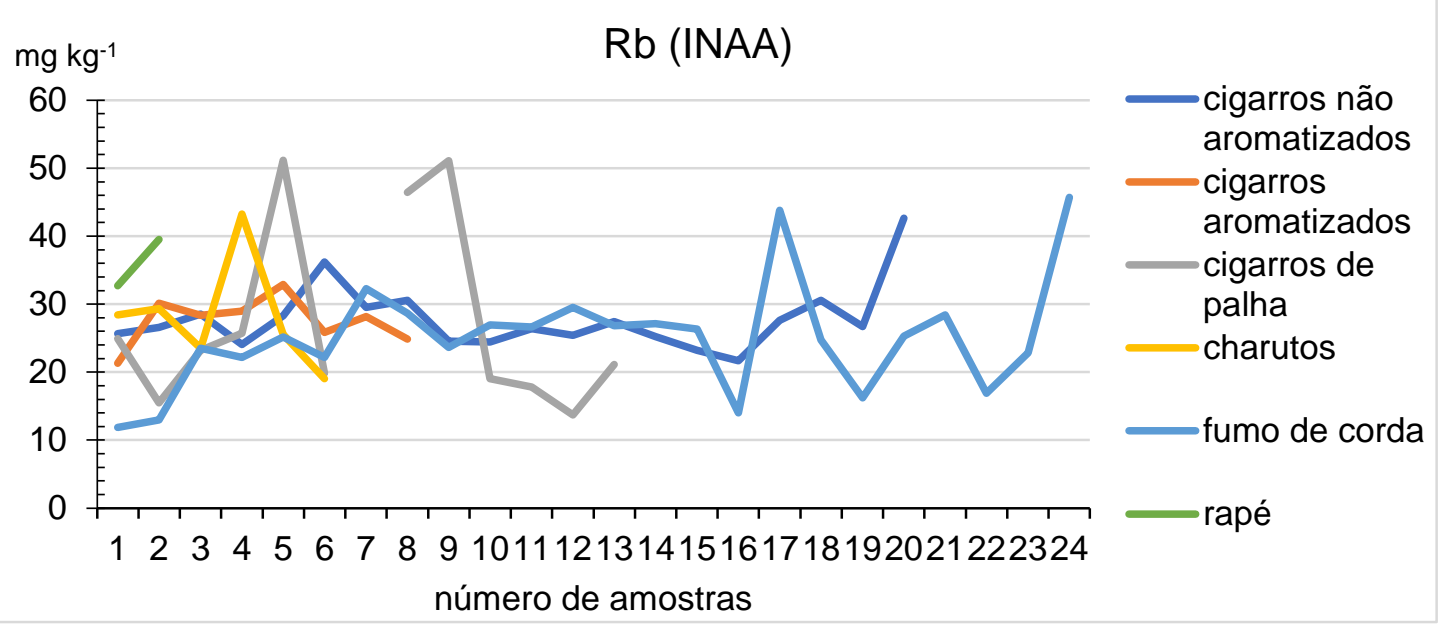

A concentração do elemento $Z n$ variou de $17,1 \pm 0,5 \mathrm{mg} \mathrm{kg}^{-1}$ na amostra de fumo de corda FCACE a $137 \pm 7 \mathrm{mg} \mathrm{kg}^{-1}$ na amostra de cigarro de palha CPFN (Apêndice A). O elemento Zn apresentou significante diferença entre o grupo de cigarros industrialmente processados (cigarros não aromatizados e aromatizados) e os menos industrializados, como os charutos, fumo de corda cigarro de palha e rapé.

FIGURA 4.19 - Variação da concentração do elemento Zn $\left(\mathrm{mg} \mathrm{kg}^{-1}\right)$ nos derivados da Nicotiana tabacum L. pela técnica INAA.

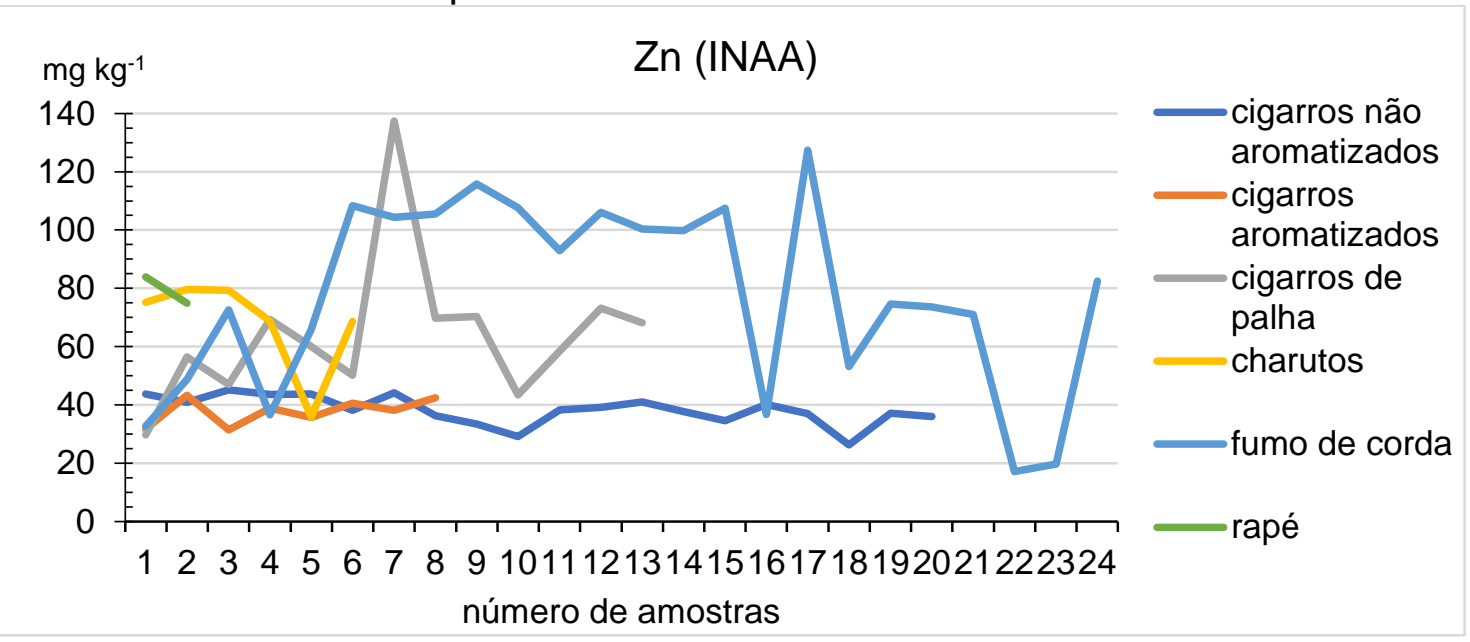

A concentração do elemento Th variou de 0,38 $\pm 0,03 \mathrm{mg} \mathrm{kg}^{-1}$ a 1,6 4 0,1 mg kg-1, ambos os valores nas amostras de cigarro de palha CPP e CPVC, respectivamente. 
FIGURA 4.20 - Variação da concentração do elemento Th $\left(\mathrm{mg} \mathrm{kg}^{-1}\right)$ nos derivados da Nicotiana tabacum L. pela técnica INAA.

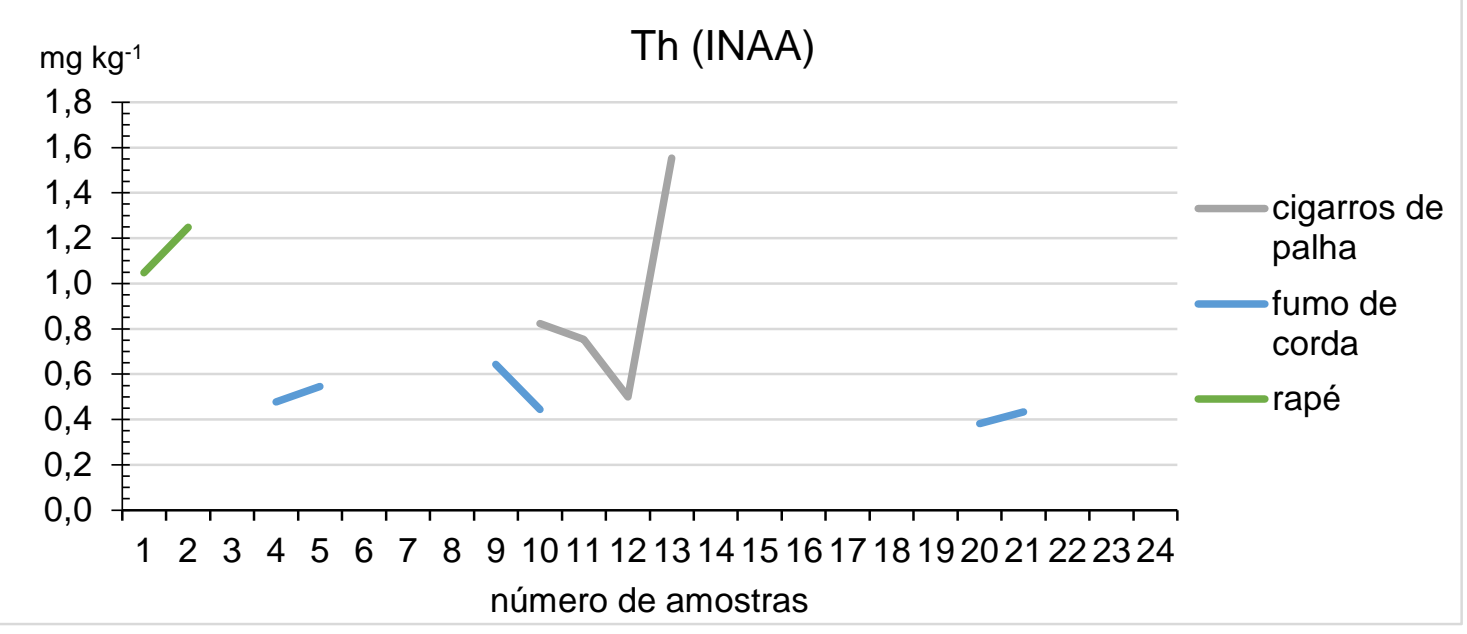

Os elementos traço, $\mathrm{Ba}, \mathrm{Rb}$ e $\mathrm{Zn}$, foram os elementos que apresentaram os maiores valores médios de concentração em todas as amostras analisadas.

\subsubsection{Elementos Terras Raras Ce, Eu, La, Lu, Nd, Sc, Sm, Tb e Yb}

Na TAB. 4.15, são apresentados os valores mínimos, máximos e médios das concentrações dos elementos Ce, La, Sc e Sm em mg kg-1 nas amostras de cigarros não aromatizados, cigarros aromatizados, cigarros de palha, charutos, fumos de corda e rapé.

Os elementos Lu e Nd apresentaram concentrações abaixo do LID, $0,05 \mathrm{mg} \mathrm{kg}^{-1}$ e $32 \mathrm{mg} \mathrm{kg}^{-1}$, respectivamente. 


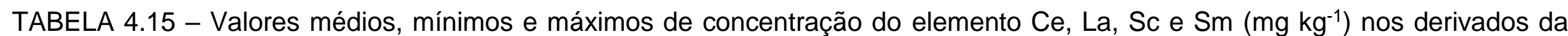
Nicotiana tabacum L., pela técnica de INAA

\begin{tabular}{|c|c|c|c|c|c|}
\hline DERIVADOS & & CE & LA & SC & SM \\
\hline \multirow{3}{*}{$\begin{array}{l}\text { CIGARRO NÃO } \\
\text { AROMATIZADO }\end{array}$} & Min. & $1,1 \pm 0,1$ & $1,22 \pm 0,03$ & $0,13 \pm 0,01$ & $0,079 \pm 0,004$ \\
\hline & Max. & $5,7 \pm 0,7$ & $2,7 \pm 0,1$ & $0,35 \pm 0,01$ & $0,23 \pm 0,02$ \\
\hline & Med. & $2,9 \pm 1,3$ & $1,92 \pm 0,40$ & $0,24 \pm 0,05$ & $0,15 \pm 0,04$ \\
\hline \multirow{3}{*}{ CIGARRO AROMATIZADO } & Min. & $0,9 \pm 0,2$ & $1,4 \pm 0,1$ & $0,16 \pm 0,01$ & $0,077 \pm 0,001$ \\
\hline & Max. & $2,3 \pm 0,3$ & $3,8 \pm 0,2$ & $0,29 \pm 0,01$ & $0,17 \pm 0,01$ \\
\hline & Med. & $1,8 \pm 0,5$ & $2,4 \pm 0,9$ & $0,23 \pm 0,05$ & $0,11 \pm 0,03$ \\
\hline \multirow{3}{*}{ CIGARRO DE PALHA } & Min. & $1,7 \pm 0,2$ & $1,3 \pm 0,04$ & $0,12 \pm 0,004$ & $0,115 \pm 0,004$ \\
\hline & Max. & $11 \pm 1$ & $31 \pm 1$ & $1,29 \pm 0,04$ & $0,84 \pm 0,003$ \\
\hline & Med. & $6,1 \pm 2,7$ & $10,1 \pm 4,8$ & $0,49 \pm 0,39$ & $0,41 \pm 0,21$ \\
\hline \multirow{3}{*}{ CHARUTO } & Min. & $1,8 \pm 0,2$ & $<0,53$ & $0,085 \pm 0,002$ & $<0,04$ \\
\hline & Max. & $1,9 \pm 0,2$ & $0,89 \pm 0,03$ & $0,730 \pm 0,003$ & $<0,04$ \\
\hline & Med. & $1,8 \pm 0,1$ & $0,79 \pm 0,09$ & $0,10 \pm 0,02$ & $<0,04$ \\
\hline \multirow{3}{*}{ FUMO DE CORDA } & Min. & $1,2 \pm 0,2$ & $0,79 \pm 0,02$ & $0,05 \pm 0,002$ & $0,04 \pm 0,002$ \\
\hline & Max. & $25 \pm 2$ & $72 \pm 2$ & $0,61 \pm 0,02$ & $0,8 \pm 0,02$ \\
\hline & Med. & $5,4 \pm 5,6$ & $11,0 \pm 17,6$ & $0,23 \pm 0,13$ & $0,30 \pm 0,26$ \\
\hline \multirow{3}{*}{ RAPÉ } & Min. & $6,8 \pm 0,6$ & $8,6 \pm 0,2$ & $0,58 \pm 0,02$ & $0,40 \pm 0,01$ \\
\hline & Max. & $8,0 \pm 0,7$ & $8,7 \pm 0,2$ & $1,01 \pm 0,03$ & $0,50 \pm 0,02$ \\
\hline & Med. & $7,4 \pm 0,9$ & $8,63 \pm 0,03$ & $0,79 \pm 0,30$ & $0,45 \pm 0,07$ \\
\hline
\end{tabular}


O elemento Eu apresentou concentrações detectáveis nos grupos de cigarro de palha e de fumo de corda, TAB. 4.16; o elemento Tb nos grupos de cigarro não aromatizado, cigarro de palha e fumo de corda, TAB. 4.17 e o elemento Yb nos grupos de cigarro de palha e fumo de corda, TAB. 4.18.

TABELA 4.16 - Concentração do elemento Eu $\left(\mathrm{mg} \mathrm{kg}^{-1}\right)$ nas amostras de cigarro de palha, fumo de corda e rapé pela técnica de INAA

\begin{tabular}{cccc}
\hline \multicolumn{2}{c}{ Cigarro de palha } & \multicolumn{2}{c}{ Fumo de corda } \\
\hline CPP & $0,13 \pm 0,03$ & FCP & $0,16 \pm 0,03$ \\
\hline CPTV & $0,2 \pm 0,1$ & FCPF & $0,15 \pm 0,03$ \\
\hline CPPG & $0,10 \pm 0,05$ & FCA4 & $0,14 \pm 0,03$ \\
\hline CPVC & $0,15 \pm 0,03$ & FCA5 & $0,12 \pm 0,02$ \\
\hline Média \pm DP & $\mathbf{0 , 1 5} \pm \mathbf{0 , 0 1}$ & FCC & $0,10 \pm 0,02$ \\
\hline & FCG & $0,11 \pm 0,02$ \\
\hline & FCDMP & $0,11 \pm 0,02$ \\
\hline & & Média \pm DP & $\mathbf{0 , 1 3} \pm \mathbf{0 , 0 2}$ \\
\hline
\end{tabular}

TABELA 4.17 - Concentração do elemento $\mathrm{Tb}\left(\mathrm{mg} \mathrm{kg}^{-1}\right)$ nas amostras de cigarro de palha, fumo de corda e rapé pela técnica de INAA

\begin{tabular}{|c|c|c|c|c|c|}
\hline \multicolumn{2}{|c|}{$\begin{array}{l}\text { Cigarro não } \\
\text { aromatizado }\end{array}$} & \multicolumn{2}{|c|}{ Cigarro de palha } & \multicolumn{2}{|c|}{ Fumo de corda } \\
\hline CCFA & $0,10 \pm 0,03$ & CPP & $0,14 \pm 0,05$ & FCA4 & $0,05 \pm 0,02$ \\
\hline & & CPCR & $0,06 \pm 0,02$ & FCA5 & $0,12 \pm 0,03$ \\
\hline & & CPVC & $0,14 \pm 0,05$ & FCDD & $0,08 \pm 0,02$ \\
\hline & & Média \pm DP & $0,10 \pm 0,05$ & Média \pm DP & $0,09 \pm 0,03$ \\
\hline
\end{tabular}

TABELA 4.18 - Concentração do elemento $\mathrm{Yb}\left(\mathrm{mg} \mathrm{kg}^{-1}\right)$ nas amostras de cigarro de palha, fumo de corda e rapé pela técnica de INAA

\section{Cigarro de palha Fumo de corda}

$\begin{array}{llll}\text { CPVC } & 0,39 \pm 0,04 & \text { FCA4 } & 0,32 \pm 0,04\end{array}$

A concentração do elemento Ce variou de 0,9 $\pm 0,2 \mathrm{mg} \mathrm{kg}^{-1}$ na amostra de cigarro aromatizado CALAM a $25 \pm 2 \mathrm{mg} \mathrm{kg}^{-1}$ na amostra de fumo de corda, FCPF. Na FIG 4.21 pode-se observar as variações das concentrações de Ce em 
todo grupo amostral; o grupo de amostras que apresentou a maior variação nas concentrações do elemento Ce foi o de fumo de corda.

FIGURA 4.21 - Variação da concentração do elemento Ce $\left(\mathrm{mg} \mathrm{kg}^{-1}\right)$ nos derivados da Nicotiana tabacum L. pela técnica INAA.

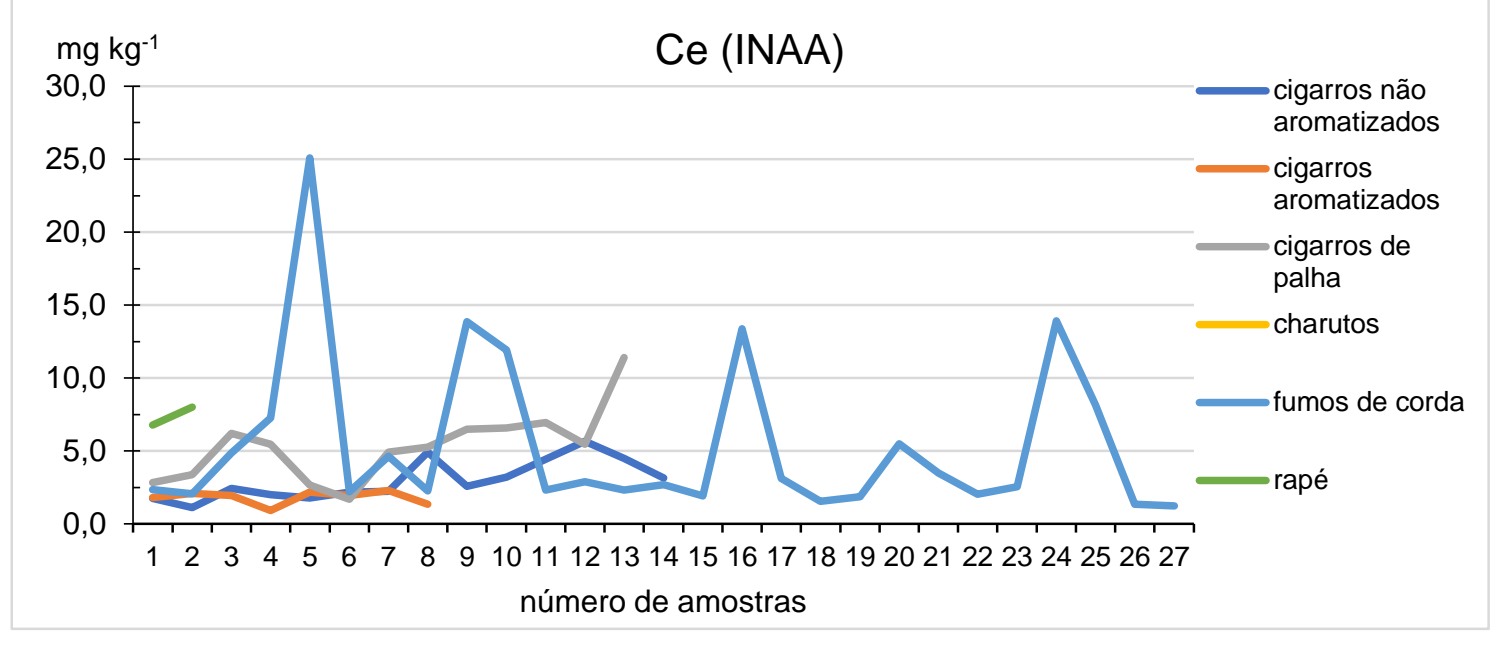

A concentração do elemento La variou $0,79 \pm 0,02 \mathrm{mg} \mathrm{kg}^{-1}$ na amostra de fumo de corda $\mathrm{FCl}$ a $31 \pm 1 \mathrm{mg} \mathrm{kg}^{-1}$ na amostra de cigarro de palha CPP. Na FIG. 4.22 é apresentada a variação das concentrações do elemento La nos derivados de tabaco estudados. Pode-se observar que o grupo de amostras que apresentou maiores variações nas concentrações determinadas foi o de fumo de corda.

FIGURA 4.22 - Variação da concentração do elemento La $\left(\mathrm{mg} \mathrm{kg}^{-1}\right)$ nos derivados da Nicotiana tabacum L. pela técnica INAA.

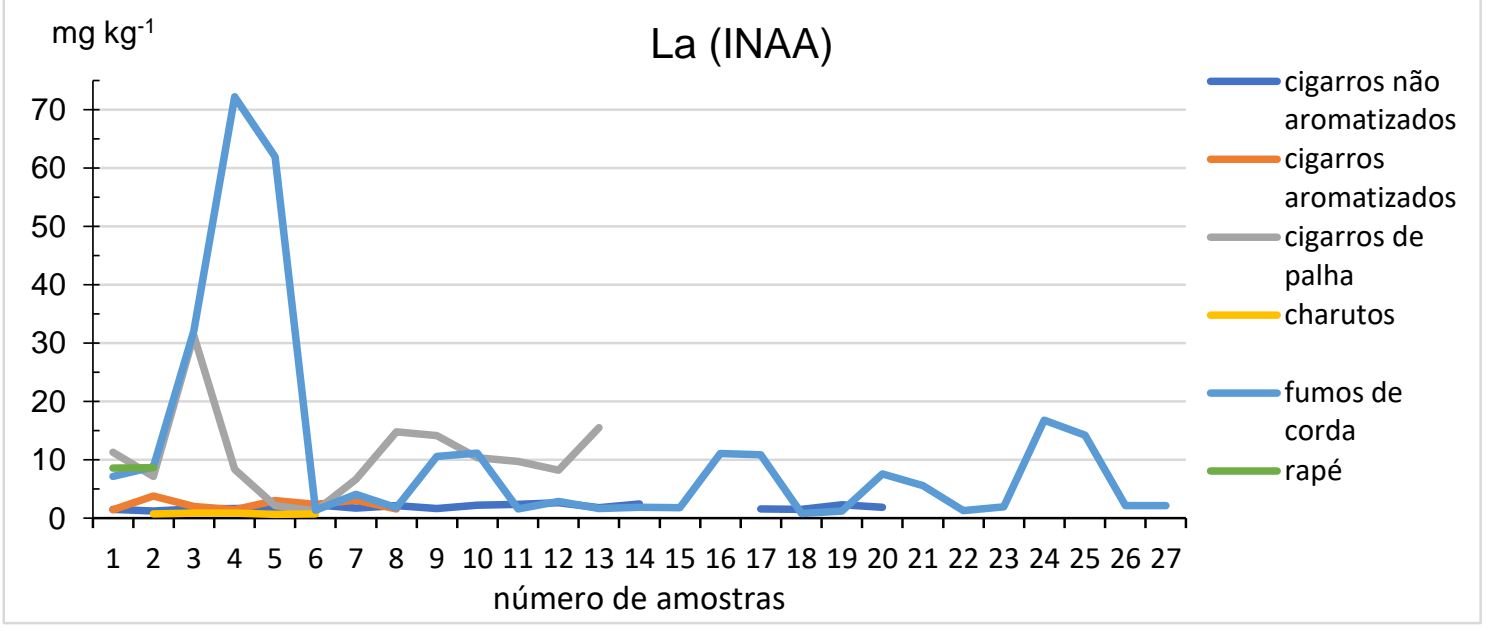


A concentração do elemento Sc de 0,085 \pm 0,002 $\mathrm{mg} \mathrm{kg}^{-1}$ na amostra de charuto CHMPE a 1,29 $\pm 0,04 \mathrm{mg} \mathrm{kg}^{-1}$ na amostra de cigarro de palha CPVC. $\mathrm{Na}$ FIG. 4.23 é apresentada a variação das concentrações do elemento Sc nos derivados de tabaco estudados.

FIGURA 4.23 - Variação da concentração do elemento Sc $\left(\mathrm{mg} \mathrm{kg}^{-1}\right)$ nos derivados da Nicotiana tabacum L. pela técnica INAA.

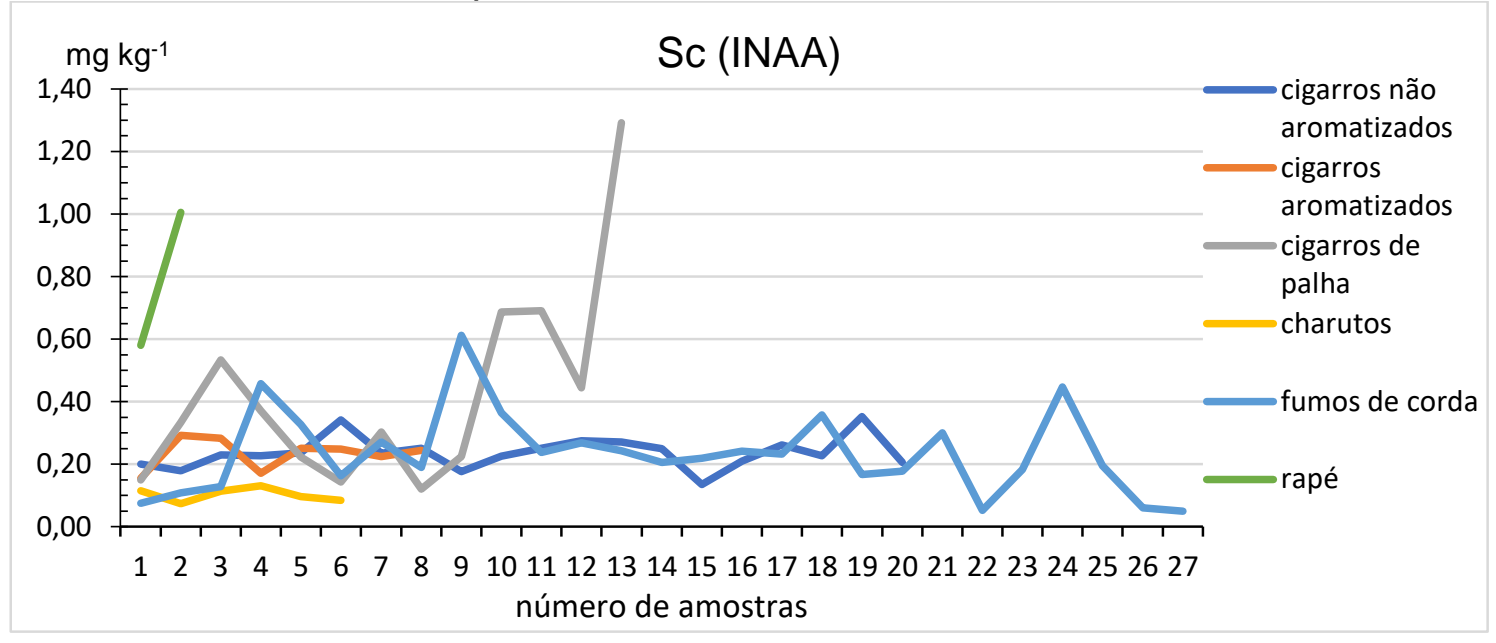

Segundo KABATA-PENDIAS (2010) plantas de solos arenosos apresentam concentrações maiores do elemento Sc em sua composição, assim como as folhas velhas apresentam concentrações superiores as das folhas jovens. Os autores relatam alguns estudos que determinaram o intervalo de concentração de Sc em algumas folhas de plantas, como folhas de alface, 0,007 $\mathrm{mg} \mathrm{kg}^{-1}$ a 0,012 $\mathrm{mg} \mathrm{kg}{ }^{-1}$ e folhas de chá de diferentes paises $0,013 \mathrm{mg} \mathrm{kg}^{-1}$ a $0,14 \mathrm{mg} \mathrm{kg}^{-1}$. A concentração do elemento Sc nas amostras analisadas no presente trabalho variaram de 0,05 $\mathrm{mg} \mathrm{kg}^{-1}$ a 1,29 $\mathrm{mg} \mathrm{kg}^{-1}$, amostras de fumo de corda aromatizado (FCACE e FCAMO) e cigarro de palha CPVC, respectivamente.

Os charutos foram o grupo de amostras que apresentaram menores valores de concentração para o elemento Sc e os grupos que apresentaram maiores variações nas concentrações foram os grupo de cigarro de palha e de fumo de corda.

A concentração do elemento Sm variou de 0,041 $\pm 0,002 \mathrm{mg} \mathrm{kg}^{-1} \mathrm{na}$ amostra de fumo de corda FCAAL a 0,80 $\pm 0,03 \mathrm{mg} \mathrm{kg}^{-1}$ na amostra de fumo de 
corda FCA5. Na FIG. 4.24 é apresentada a variação das concentrações do elemento Sm nos derivados de tabaco estudados. Pode-se observar uma grande variação na concentração do elemento Sm nas amostras do grupo de fumo de corda e cigarro de palha.

FIGURA 4.24 - Variação da concentração do elemento Sm $\left(\mathrm{mg} \mathrm{kg}^{-1}\right)$ nos derivados da Nicotiana tabacum L. pela técnica INAA.

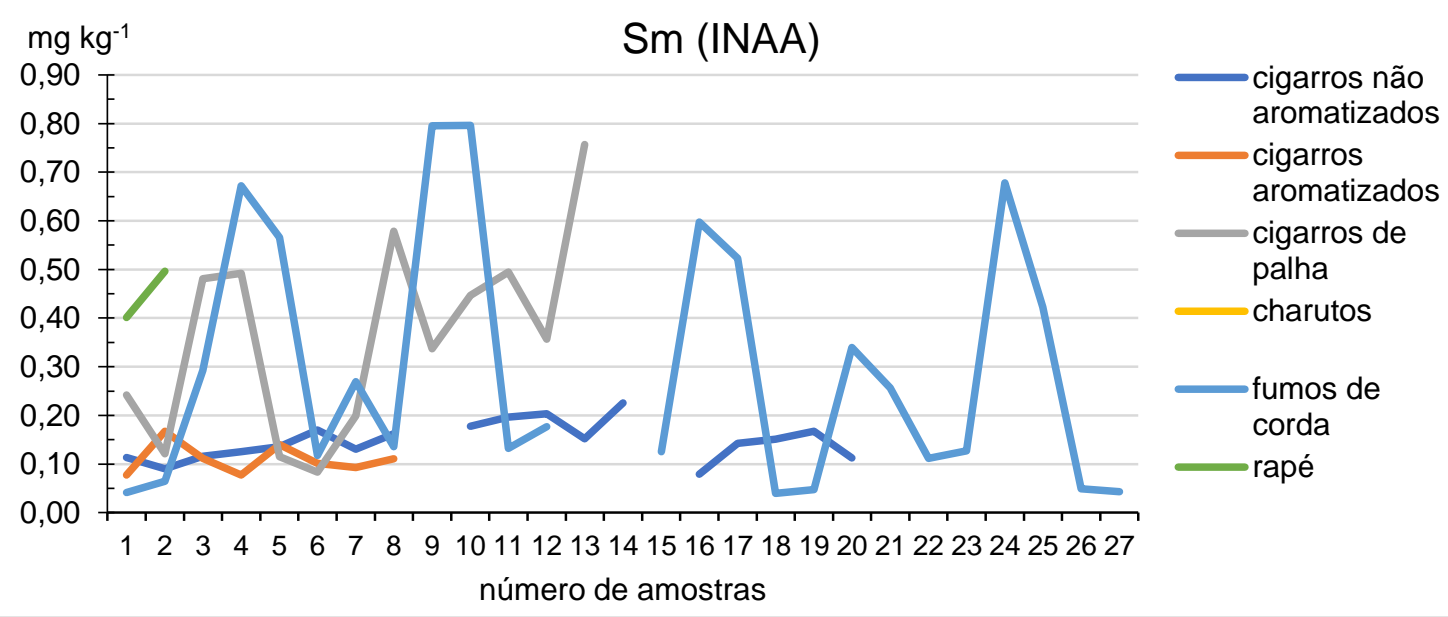

$\mathrm{Na}$ Tab. 4.19 são apresentados os intervalos de concentração dos elementos de terras raras determinados no presente trabalho e em amostras de vegetais e palha de arroz apresentadas por KABATA-PENDIAS (2010). Pode-se observar que os resultados obtidos no presente trabalho estão dentro da faixa de concentração dos elementos determinados nos vegetais.

Tabela 4.19 - Intervalo de concentração de elementos terras raras, $\mathrm{mg} \mathrm{kg}^{-1}$, presente trabalho e de Kabata-Pendias (2010)

\begin{tabular}{cccc}
$\begin{array}{c}\text { Elementos } \\
\text { Terras Raras }\end{array}$ & $\begin{array}{c}\text { Derivados de tabaco } \\
\text { presente trabalho }\end{array}$ & $\begin{array}{c}\text { Vegetais } \\
\text { (KABATA-PENDIAS, } \\
\mathbf{2 0 1 0 )}\end{array}$ & $\begin{array}{c}\text { Palha de arroz } \\
\text { (KABATA-PENDIAS, } \\
\text { 2010) }\end{array}$ \\
\hline Ce & $0,9-25$ & $0,2-50$ & 174 \\
\hline La & $0,79-72$ & $0,4-2000$ & 88 \\
\hline Nd & - & 50 & 10 \\
\hline Sc & $0,05-1,29$ & $0,007-0,14$ & 20 \\
\hline Sm & $0,04-0,84$ & $0,2-100$ & 10 \\
\hline Eu & $0,10-0,20$ & $0,04-70$ & 7 \\
\hline Tb & $0,05-0,14$ & $0,1-1$ & 10 \\
\hline Yb & $0,32-0,39$ & $0,08-20$ & 5
\end{tabular}

- não detectado

* não informado 
Abd-El-Samad e Hanafi (2017) analisaram 10 marcas de cigarros egípcios pela técnica de INAA e determinaram a concentração de 14 elementos (Ba, Br, Ca, Cd, Eu, K, Hf, Mg, Na, Rb, Sb, Sc, Th e Yb). TAB. 4.20 apresenta os valores determinados pelos autores e do presente estudo. Verifica-se que a maioria dos elementos estudados apresentaram concentrações médias menores que as determinada pelos autores, com exceção do elemento sódio e para a maioria dos elementos não houve diferença significativa entre os grupos de cigarros não aromatizados e aromatizados.

Tabela 4.20 - Concentrações médias em mg kg-1 (*\%) no presente trabalho e de cigarros egípcios pela técnica INAA.

\begin{tabular}{c|c|cc}
\hline \multirow{2}{*}{ Elementos } & $\begin{array}{c}\text { ABD-EL-SAMAD E HANAFI } \\
(2017)\end{array}$ & \multicolumn{2}{|c}{ Presente estudo } \\
\hline $\mathrm{Ba}$ & Cigarros egípcios & $\begin{array}{c}\text { Cigarros não } \\
\text { aromatizados }\end{array}$ & $\begin{array}{c}\text { Cigarros } \\
\text { aromatizados }\end{array}$ \\
\hline $\mathrm{Br}$ & 149 & 140 & 121 \\
\hline${ }^{*} \mathrm{Ca}$ & 184 & 56 & 49 \\
\hline $\mathrm{Eu}$ & 9,5 & 3,1 & 2,4 \\
\hline${ }^{*}$ & 0,16 & $<0,09$ & $<0,09$ \\
\hline $\mathrm{Hf}$ & 4,5 & 3,5 & 0,22 \\
\hline $\mathrm{Na}$ & 1,0 & 0,21 & 879 \\
\hline $\mathrm{Rb}$ & 553 & 688 & 28 \\
\hline $\mathrm{Sb}$ & 45 & 28 & $<0,21$ \\
\hline $\mathrm{Sc}$ & 1,3 & $<0,21$ & 0,23 \\
\hline $\mathrm{Th}$ & 0,5 & 0,24 & $<0,38$ \\
\hline $\mathrm{Yb}$ & 0,6 & $<0,38$ & $<0,30$
\end{tabular}

\subsubsection{Espectrometria de Fluorescência de Raios-X (EDXRF)}

\subsubsection{Elementos maiores $\mathrm{Al}, \mathrm{Ca}, \mathrm{Cl}, \mathrm{Fe}, \mathrm{K}, \mathrm{I}, \mathrm{Mg}, \mathrm{Mn}, \mathrm{P}$ e $\mathrm{S}$}

Na TAB. 4.21 são apresentados os resultados das concentrações mínimos, máximos e médios para os elementos $\mathrm{Al}, \mathrm{Ca}, \mathrm{Cl}, \mathrm{Fe}, \mathrm{K}, \mathrm{I}, \mathrm{Mg}, \mathrm{Mn}, \mathrm{P}$ e S em \% nas amostras de cigarros não aromatizados, cigarros aromatizados, cigarros de palha, charutos, fumos de corda e rapé determinados por espectrometria de fluorescência de raios $X$. 
Tabela 4.21 - Valores médios, mínimos e máximos de concentração dos elementos $\mathrm{Al}, \mathrm{Ca}, \mathrm{Cl}, \mathrm{Fe}, \mathrm{K}, \mathrm{I}, \mathrm{Mg}, \mathrm{Mn}, \mathrm{P}$ e S em \% nos derivados da Nicotiana tabacum L., pela técnica de EDXRF.

\begin{tabular}{|c|c|c|c|c|c|c|c|c|c|c|c|}
\hline DERIVADOS & & $\mathrm{AL}$ & CA & CL & FE & I & $\mathrm{K}$ & MG & MN & $\mathbf{P}$ & $S$ \\
\hline \multirow{3}{*}{$\begin{array}{c}\text { CIGARROS } \\
\text { NÃO } \\
\text { AROMATIZAD } \\
\text { OS }\end{array}$} & Min. & $0,041 \pm 0,004$ & $1,45 \pm 0,01$ & $0,48 \pm 0,01$ & $0,040 \pm 0,001$ & $0,019 \pm 0,004$ & $2,02 \pm 0,01$ & $0,53 \pm 0,02$ & $0,011 \pm 0,001$ & $0,11 \pm 0,01$ & $0,241 \pm 0,001$ \\
\hline & Max. & $0,094 \pm 0,003$ & $1,82 \pm 0,03$ & $1,29 \pm 0,01$ & $0,086 \pm 0,003$ & $0,022 \pm 0,006$ & $3,54 \pm 0,01$ & $1,22 \pm 0,04$ & $0,026 \pm 0,001$ & $0,22 \pm 0,01$ & $0,332 \pm 0,002$ \\
\hline & Med. & $0,065 \pm 0,015$ & $1,67 \pm 0,09$ & $0,78 \pm 0,23$ & $0,058 \pm 0,013$ & $0,021 \pm 0,001$ & $2,87 \pm 0,41$ & $0,87 \pm 0,18$ & $0,019 \pm 0,005$ & $0,17 \pm 0,03$ & $0,290 \pm 0,020$ \\
\hline \multirow{3}{*}{$\begin{array}{l}\text { CIGARROS } \\
\text { AROMATIZAD } \\
\text { OS }\end{array}$} & Min. & $0,045 \pm 0,005$ & $1,60 \pm 0,03$ & $0,550 \pm 0,004$ & $0,047 \pm 0,001$ & $0,020 \pm 0,001$ & $2,40 \pm 0,01$ & $0,67 \pm 0,01$ & $0,015 \pm 0,002$ & $0,14 \pm 0,01$ & $0,253 \pm 0,003$ \\
\hline & Max. & $0,085 \pm 0,004$ & $1,83 \pm 0,05$ & $1,095 \pm 0,004$ & $0,080 \pm 0,002$ & $0,022 \pm 0,001$ & $3,31 \pm 0,01$ & $1,07 \pm 0,04$ & $0,023 \pm 0,001$ & $0,18 \pm 0,01$ & $0,336 \pm 0,001$ \\
\hline & Med. & $0,067 \pm 0,016$ & $1,75 \pm 0,09$ & $0,833 \pm 0,200$ & $0,059 \pm 0,010$ & $0,021 \pm 0,001$ & $2,77 \pm 0,32$ & $0,90 \pm 0,15$ & $0,019 \pm 0,003$ & $0,16 \pm 0,02$ & $0,288 \pm 0,027$ \\
\hline \multirow{3}{*}{$\begin{array}{c}\text { CIGARROS DE } \\
\text { PALHA }\end{array}$} & Min. & $0,048 \pm 0,003$ & $1,32 \pm 0,02$ & $0,117 \pm 0,005$ & $0,027 \pm 0,001$ & $0,016 \pm 0,002$ & $0,93 \pm 0,01$ & $0,31 \pm 0,04$ & $0,008 \pm 0,001$ & $0,082 \pm 0,004$ & $0,252 \pm 0,003$ \\
\hline & Max. & $0,25 \pm 0,01$ & $3,41 \pm 0,03$ & $1,30 \pm 0,01$ & $0,202 \pm 0,004$ & $0,024 \pm 0,001$ & $3,66 \pm 0,01$ & $1,33 \pm 0,05$ & $0,081 \pm 0,006$ & $0,22 \pm 0,01$ & $0,45 \pm 0,01$ \\
\hline & Med. & $0,091 \pm 0,052$ & $2,43 \pm 0,59$ & $0,545 \pm 0,300$ & $0,094 \pm 0,040$ & $0,021 \pm 0,002$ & $1,94 \pm 0,85$ & $0,72 \pm 0,30$ & $0,025 \pm 0,020$ & $0,123 \pm 0,046$ & $0,318 \pm 0,053$ \\
\hline \multirow{3}{*}{ CHARUTOS } & Min. & $0,056 \pm 0,003$ & $1,59 \pm 0,01$ & $0,70 \pm 0,01$ & $0,038 \pm 0,001$ & $0,019 \pm 0,001$ & $2,63 \pm 0,01$ & $1,44 \pm 0,03$ & $0,012 \pm 0,001$ & $0,16 \pm 0,01$ & $0,459 \pm 0,004$ \\
\hline & Max. & $0,118 \pm 0,003$ & $2,40 \pm 0,03$ & $1,47 \pm 0,01$ & $0,053 \pm 0,001$ & $0,021 \pm 0,001$ & $5,45 \pm 0,01$ & $2,41 \pm 0,03$ & $0,016 \pm 0,001$ & $0,25 \pm 0,01$ & $0,684 \pm 0,002$ \\
\hline & Med. & $0,087 \pm 0,021$ & $1,91 \pm 0,32$ & $1,12 \pm 0,30$ & $0,045 \pm 0,07$ & $0,020 \pm 0,001$ & $3,82 \pm 1,00$ & $1,92 \pm 0,37$ & $0,014 \pm 0,002$ & $0,20 \pm 0,04$ & $0,529 \pm 0,080$ \\
\hline \multirow{3}{*}{$\begin{array}{l}\text { FUMOS DE } \\
\text { CORDA }\end{array}$} & Min. & $0,011 \pm 0,001$ & $1,42 \pm 0,02$ & $0,244 \pm 0,002$ & $0,028 \pm 0,002$ & $0,0164 \pm 0,0003$ & $1,12 \pm 0,01$ & $0,12 \pm 0,02$ & $0,007 \pm 0,001$ & $0,108 \pm 0,004$ & $0,030 \pm 0,001$ \\
\hline & Max. & $0,169 \pm 0,005$ & $2,41 \pm 0,05$ & $1,74 \pm 0,01$ & $0,118 \pm 0,001$ & $0,023 \pm 0,002$ & $4,58 \pm 0,01$ & $2,2 \pm 0,1$ & $0,081 \pm 0,004$ & $0,45 \pm 0,04$ & $0,512 \pm 0,003$ \\
\hline & Med. & $0,086 \pm 0,038$ & $1,75 \pm 0,26$ & $0,734 \pm 0,370$ & $0,059 \pm 0,022$ & $0,0211 \pm 0,0016$ & $2,89 \pm 0,91$ & $1,04 \pm 0,61$ & $0,022 \pm 0,016$ & $0,159 \pm 0,071$ & $0,342 \pm 0,091$ \\
\hline \multirow{3}{*}{ RAPÉS } & Min. & $0,097 \pm 0,003$ & $1,74 \pm 0,03$ & $0,645 \pm 0,003$ & $0,144 \pm 0,005$ & $0,021 \pm 0,001$ & $2,72 \pm 0,01$ & $0,025 \pm 0,001$ & $1,26 \pm 0,04$ & $0,25 \pm 0,01$ & $0,45 \pm 0,01$ \\
\hline & Max. & $0,131 \pm 0,004$ & $1,88 \pm 0,02$ & $0,663 \pm 0,003$ & $0,147 \pm 0,004$ & $0,022 \pm 0,001$ & $3,04 \pm 0,02$ & $0,025 \pm 0,001$ & $1,39 \pm 0,04$ & $0,327 \pm 0,004$ & $0,46 \pm 0,01$ \\
\hline & Med. & $0,114 \pm 0,024$ & $1,81 \pm 0,09$ & $0,654 \pm 0,013$ & $0,145 \pm 0,002$ & $0,022 \pm 0,001$ & $2,88 \pm 0,23$ & $0,025 \pm 0,001$ & $1,33 \pm 0,09$ & $0,286 \pm 0,058$ & $0,45 \pm 0,01$ \\
\hline
\end{tabular}


A concentração elemento Al variou de 0,041 $\pm 0,004 \%$ na amostra de cigarro não aromatizado $\mathrm{CCDH}$ a $0,25 \pm 0,01 \%$ na amostra de cigarro de palha CPVC. O grupo de amostras que apresentou maior variação dos resultados de Al foi o de cigarro de palha e fumo de corda, FIG. 4.25.

FIGURA 4.25 - Variação da concentração do elemento Al (\%) nos derivados da Nicotiana tabacum L. pela técnica EDXRF.



PAPPAS (2011) realizou um levantamento das concentrações de alguns metais em amostras de cigarro; os cigarros paquistaneses apresentaram concentrações de Al entre 431 a $782 \mu \mathrm{g} \mathrm{Al} / \mathrm{g}$ tabaco e os cigarros americanos de 699 a $1200 \mu \mathrm{g} \mathrm{Al} / \mathrm{g}$ tabaco. KAZIA et al. (2009) determinaram Al em fumaça de cigarro paquistaneses e obtiveram concentrações que variaram de 0,239 $\mu \mathrm{g} /$ cigarro a $0,964 \mu \mathrm{g} /$ cigarro. EXLEY et al. (2006) em um estudo realizado com cigarros jamaicanos obtiveram concentrações do elemento Al que variaram de 0,8 $\mathrm{mg} \mathrm{g}^{-1} \mathrm{a}$ $2,6 \mathrm{mg} \mathrm{g}^{-1}$. Os valores obtidos no presente trabalho variaram de $650 \mathrm{mg} \mathrm{g}^{-1}$ a 1140

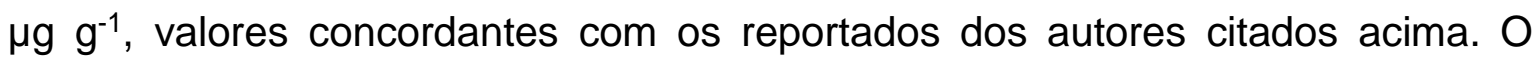
alumínio é um elemento que pode causar diversas malignidades nos tabagistas dentre elas bronquite crônica, pneumoconioses de alumínio, fibroses pulmonares, podendo até atingir o cérebro através dos caminhos olfativos (PAPPAS, 2011).

O elemento Ca variou sua concentração de 1,32 \pm 0,02 \% na amostra de cigarro de palha CPCPC a 3,41 $\pm 0,03 \%$ na amostra de cigarro de palha CPC. A FIG. 4.61 apresenta a variação da concentração do elemento Ca \% nos grupos 
amostrados; os grupos que apresentaram maior flutuação nas suas concentrações foram os de cigarros de palha e de fumos de corda.

FIGURA 4.26 - Variação da concentração do elemento Ca (\%) nos derivados da Nicotiana tabacum L. pela técnica EDXRF.

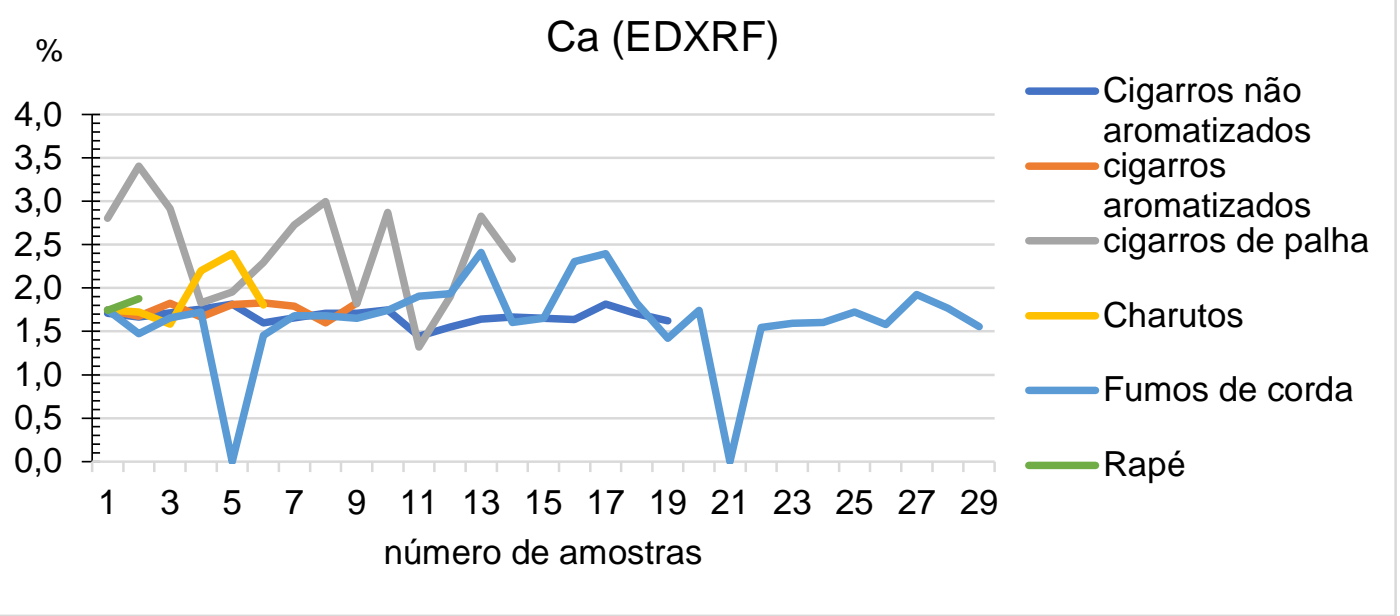

Os valores do elemento $\mathrm{Cl}$ obtidos nas amostras estão de acordo com os obtidos por MULLER et al. (2011), que determinaram a concentração do elemento em cigarros brasileiros e obtiveram concentrações que variaram de 3.871 $\mu \mathrm{g} \mathrm{g} \mathrm{g}^{-1}$ a $8.249 \mu \mathrm{g} \mathrm{g}^{-1}$, os autores citam que a concentração para o elemento pode variar de 700 a $30.000 \mu \mathrm{g} \mathrm{g}^{-1}$; as concentrações obtidas no presente trabalho variaram de $486 \mu \mathrm{g} \mathrm{g}^{-1}$ no cigarro não aromatizado CCM a $17.400 \mu^{g^{-1}}$ no fumo de corda FCA. Os autores citam ainda que o $\mathrm{Cl}$ é um elemento essencial para a planta, porém concentrações elevadas do elemento são provenientes dos agroquímicos usados nas plantações. Na FIG. 4.27 podem-se observar as variações da concentração do elemento $\mathrm{Cl}$ no grupo amostral. 
FIGURA 4.27 - Variação da concentração do elemento $\mathrm{Cl}$ (\%) nos derivados da Nicotiana tabacum L. pela técnica EDXRF.

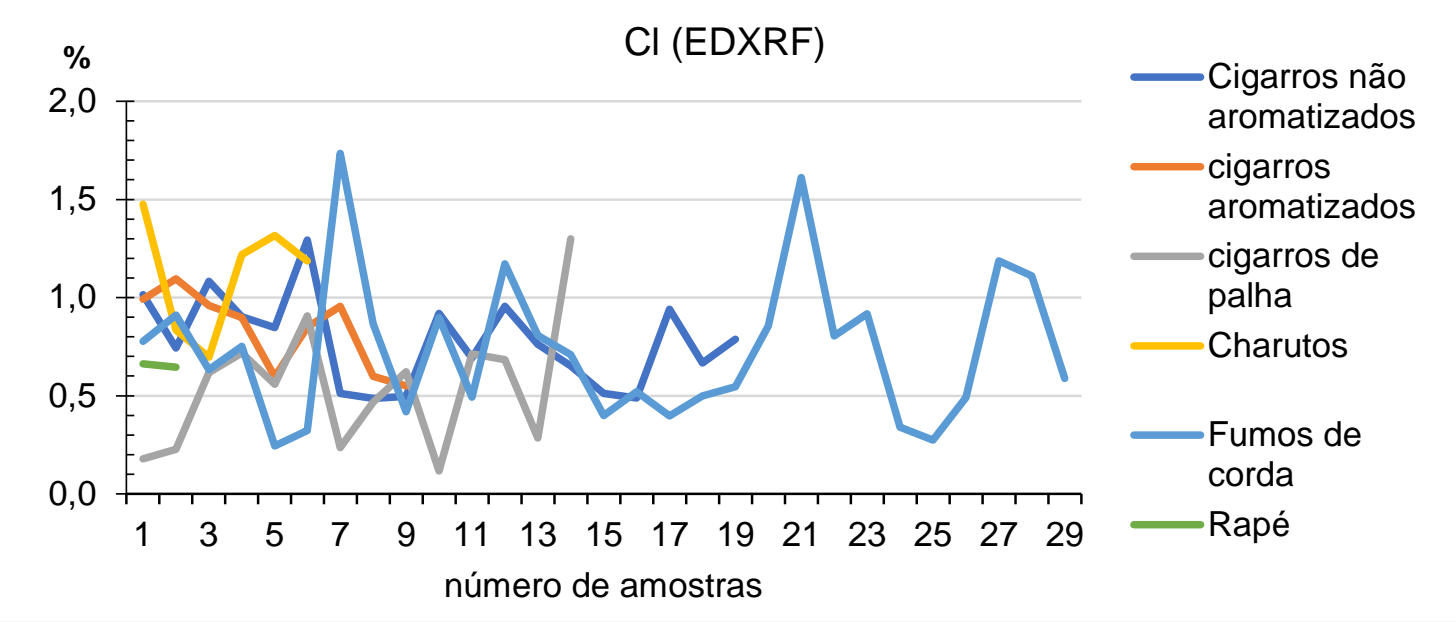

A concentração do elemento Fe variou de $283 \mu \mathrm{g} \mathrm{g}^{-1}$ na amostra de fumo de corda FCDTT a $2.019 \mu \mathrm{g} \mathrm{g}^{-1}$ na amostra de cigarro de palha CPVC; estes valores estão de acordo com os reportados por PAPPAS (2011) em cigarros indianos onde foi obtida uma variação de $468-1.129 \mu \mathrm{g} \mathrm{g}$-1 . A FIG. 4.28 apresenta a variação da concentração de Fe nas amostras analisadas e verifica-se que as maiores concentrações foram determinadas nos cigarros de palha.

FIGURA 4.28 - Variação da concentração do elemento Fe (\%) nos derivados da Nicotiana tabacum L. pela técnica EDXRF.

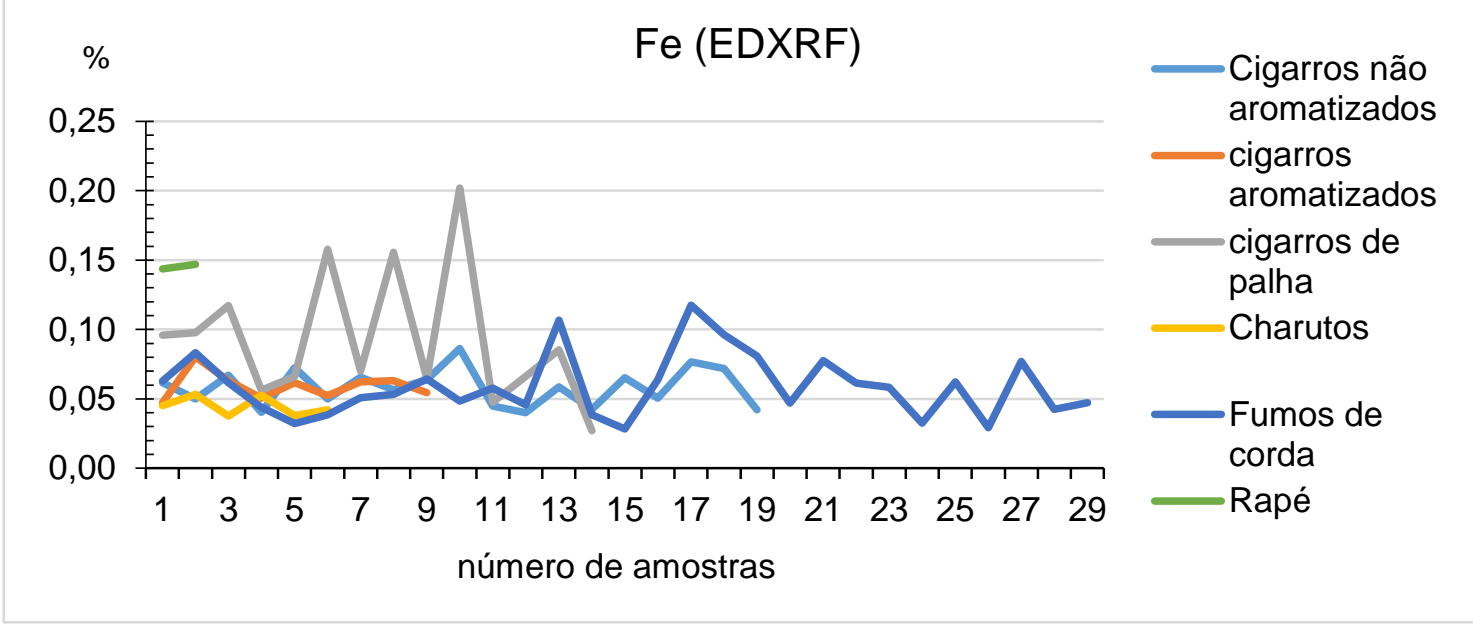

O elemento I variou sua concentração de 0,016 \pm 0,002 \% na amostra de cigarro de palha CPTV a 0,023 $\pm 0,002 \%$ na amostra de fumo de corda FCA8. A FIG. 4.24 apresenta a variação da concentração do elemento I nas amostras 
analisadas, os grupos que apresentaram maior flutuação nas suas concentrações foram os de cigarros de palha e de fumos de corda.

FIGURA 4.29 - Variação da concentração do elemento I (\%) nos derivados da Nicotiana tabacum L. pela técnica EDXRF.

$\%$

I (EDXRF)
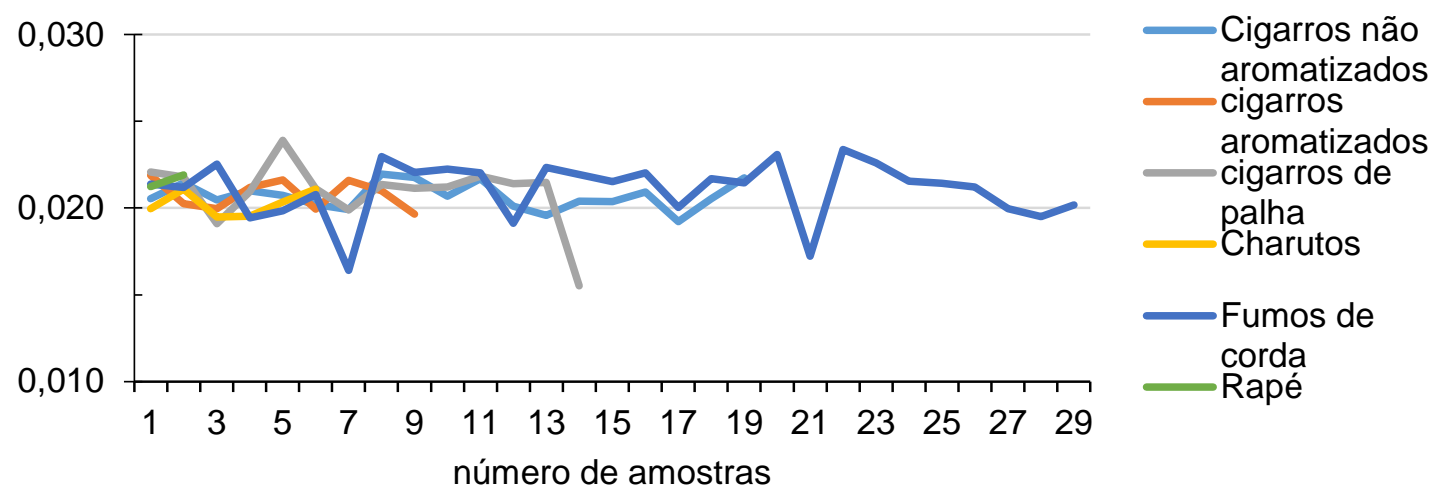

Na FIG. 4.30 observam-se as variações da concentração do elemento K; a menor concentração foi determinada na amostra de cigarro de palha CPPM de 0,93 $\pm 0,01 \%$ e a maior na amostra de charuto CHDNE de 5,45 $\pm 0,01 \%$.

FIGURA 4.30 - Variação da concentração do elemento K (\%) nos derivados da Nicotiana tabacum L. pela técnica EDXRF.

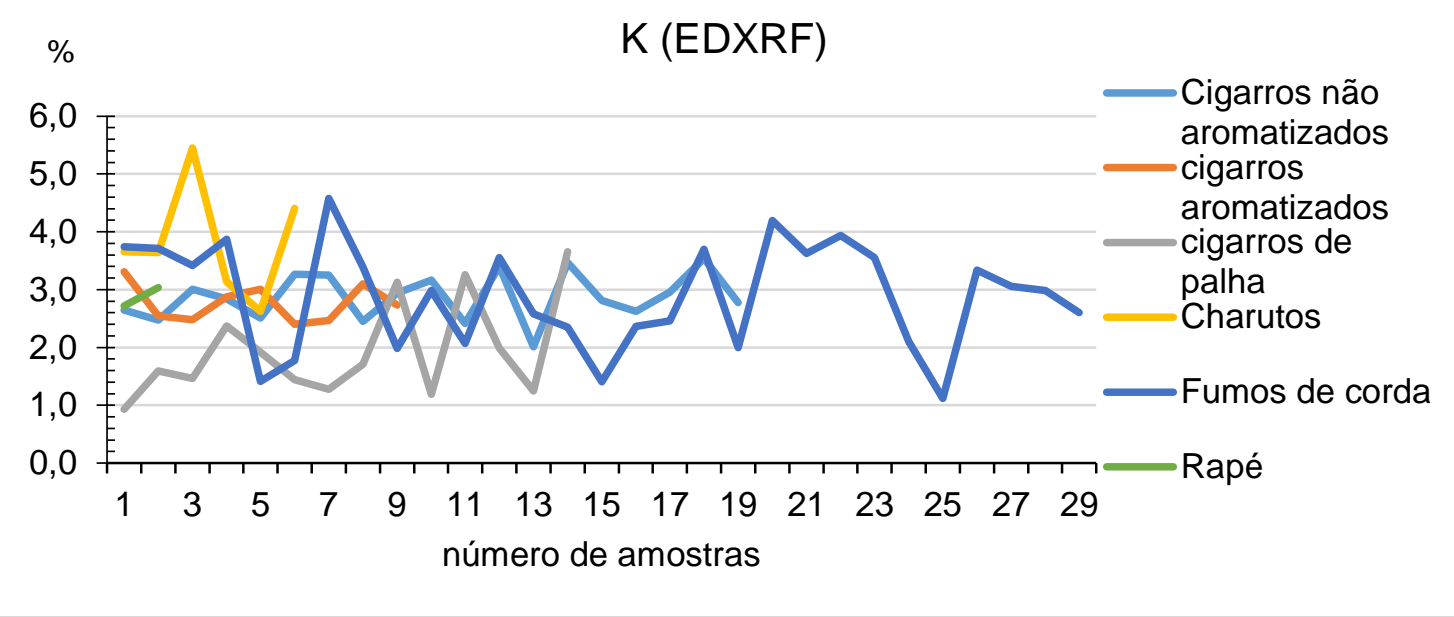

O elemento $\mathrm{Mg}$ variou sua concentração de 0,12 \pm 0,02 \% na amostra de fumo de corda FCPF a 1,39 \pm 0,04 \% na amostra de rapé RPCM. A FIG. 4.31 apresenta a variação da concentração do elemento $\mathrm{Mg}$; os grupos que 
apresentaram maior flutuação nas suas concentrações foram os de cigarros de palha e de fumos de corda.

FIGURA 4.31 - Variação da concentração do elemento Mg (\%) nos derivados da Nicotiana tabacum L. pela técnica EDXRF.

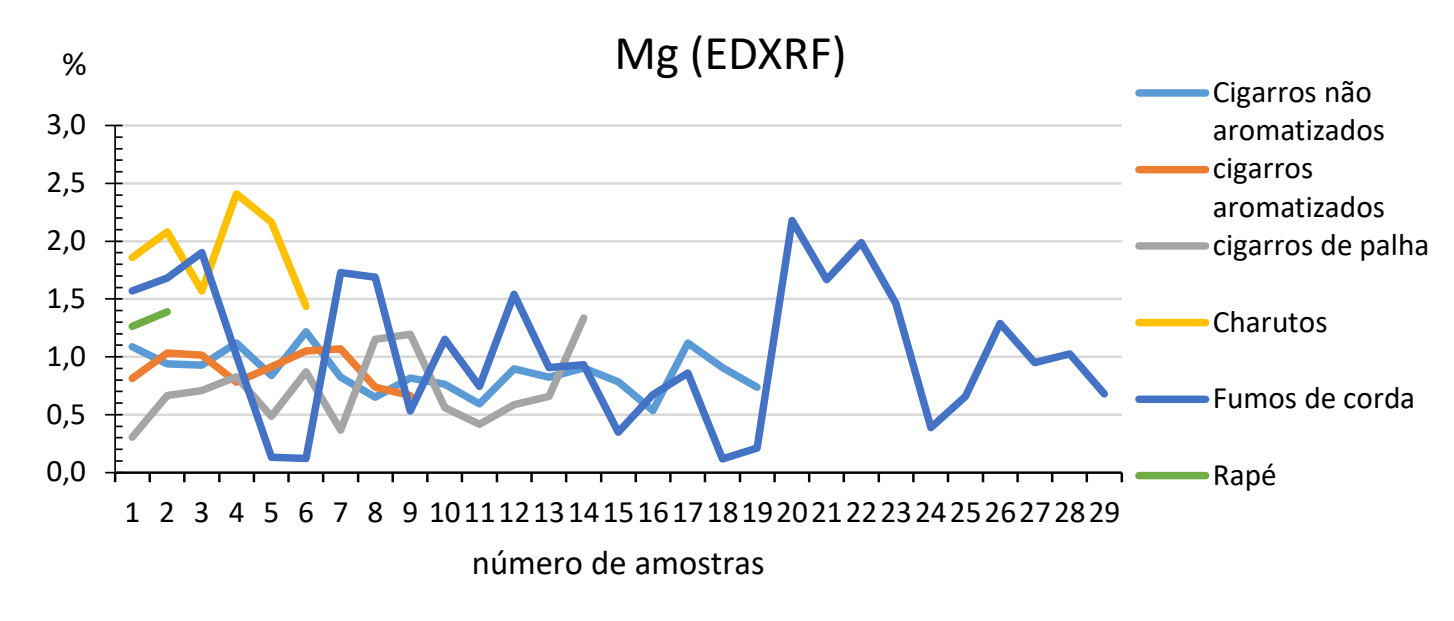

A concentração do elemento $\mathrm{Mn}$ variou de $75 \mu \mathrm{g} \mathrm{g}^{-1}$ na amostra de fumo de corda FCC a $806 \mu \mathrm{g} \mathrm{g} \mathrm{g}^{-1}$ na amostra de fumo de corda FCA4. A variação da concentração nos cigarros foi de $105 \mu \mathrm{g} \mathrm{g}^{-1}$ na amostra de cigarro não aromatizado CCM a $265 \mu \mathrm{g} \mathrm{g}^{-1}$ na amostra de cigarro não aromatizado CCPZ, estes valores são próximos aos de PAPPAS (2011) que determinou Mg em cigarros americanos, onde obteve variação de $155 \mu \mathrm{g} \mathrm{g}^{-1}-400 \mu \mathrm{g} \mathrm{g}^{-1}$. A FIG. 4.32 apresenta a variação da concentração nas amostras, verifica-se que os grupos de amostras que apresentaram maior variação nas concentrações foram os de cigarro de palha e fumo de corda. 
FIGURA 4.32 - Variação da concentração do elemento Mn (\%) nos derivados da Nicotiana tabacum L. pela técnica EDXRF.

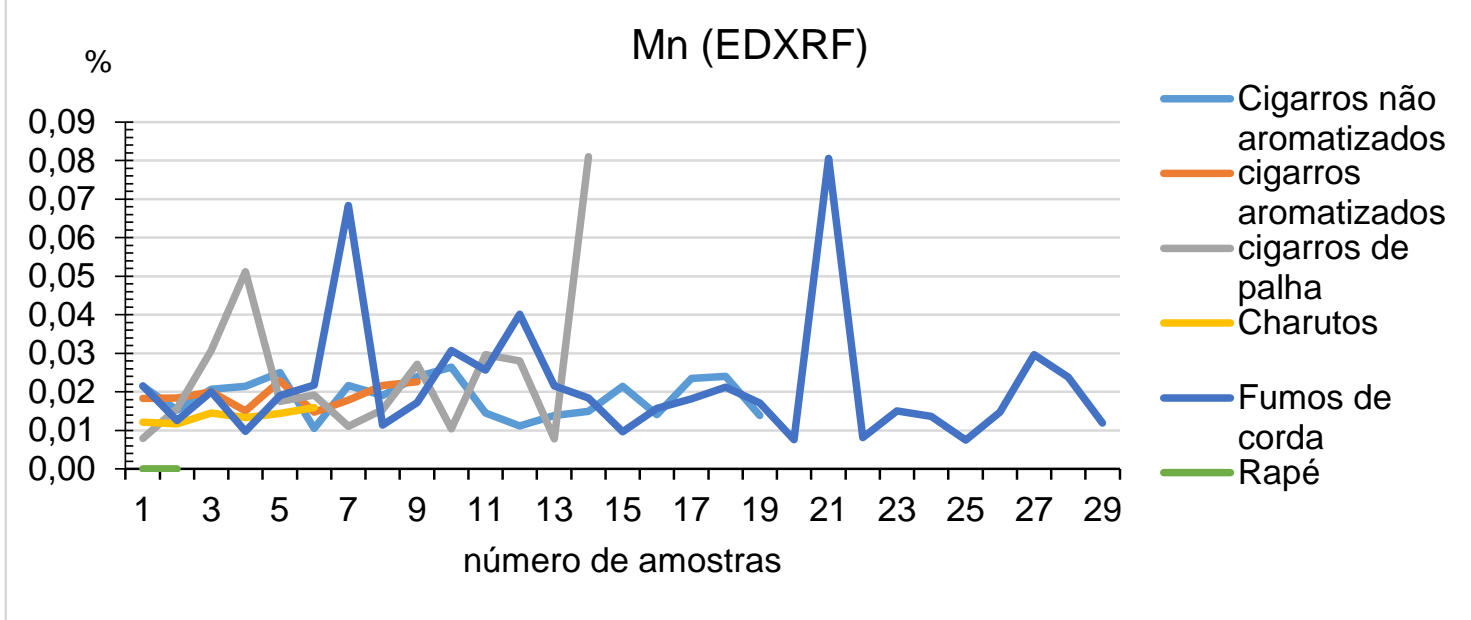

O elemento $P$ variou sua concentração de 0,108 \pm 0,004 \% na amostra de fumo de corda FCA7 a 0,45 $\pm 0,04 \%$ na amostra de fumo de corda FCCPR. A FIG. 4.33 apresenta a variação da concentração do elemento $P$ nos grupos amostrados; o grupo que apresentou maior variação nas concentrações foi o de fumo de corda.

FIGURA 4.33 - Variação da concentração do elemento P (\%) nos derivados da Nicotiana tabacum $\mathrm{L}$. pela técnica EDXRF.

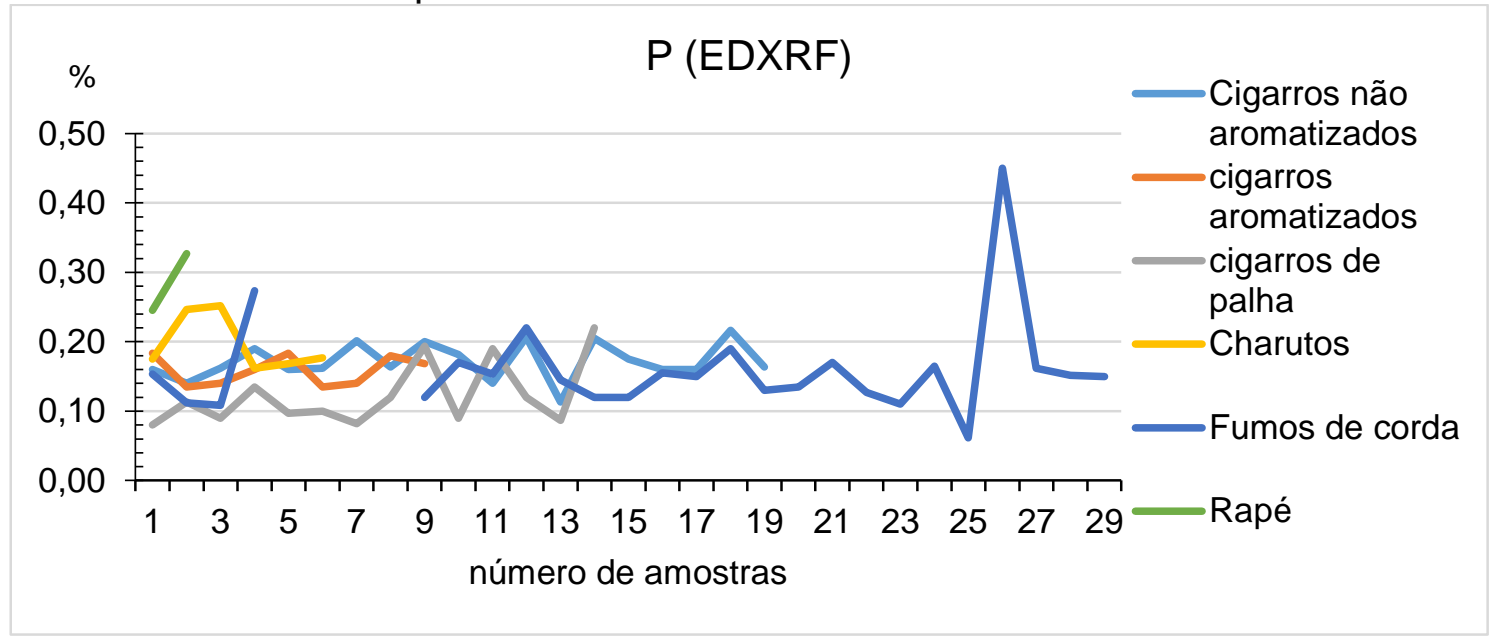

O elemento S variou sua concentração de 0,030 \pm 0,001 \% na amostra de fumo de corda FCA9 a 0,684 $\pm 0,002 \%$ na amostra de charuto CHDNE. A FIG. 4.34 apresenta a variação da concentração do elemento S nos grupos amostrados; 0 grupo que apresentou as maiores concentrações foi o de charuto. 
FIGURA 4.34 - Variação da concentração do elemento S (\%) nos derivados da Nicotiana tabacum L. pela técnica EDXRF.

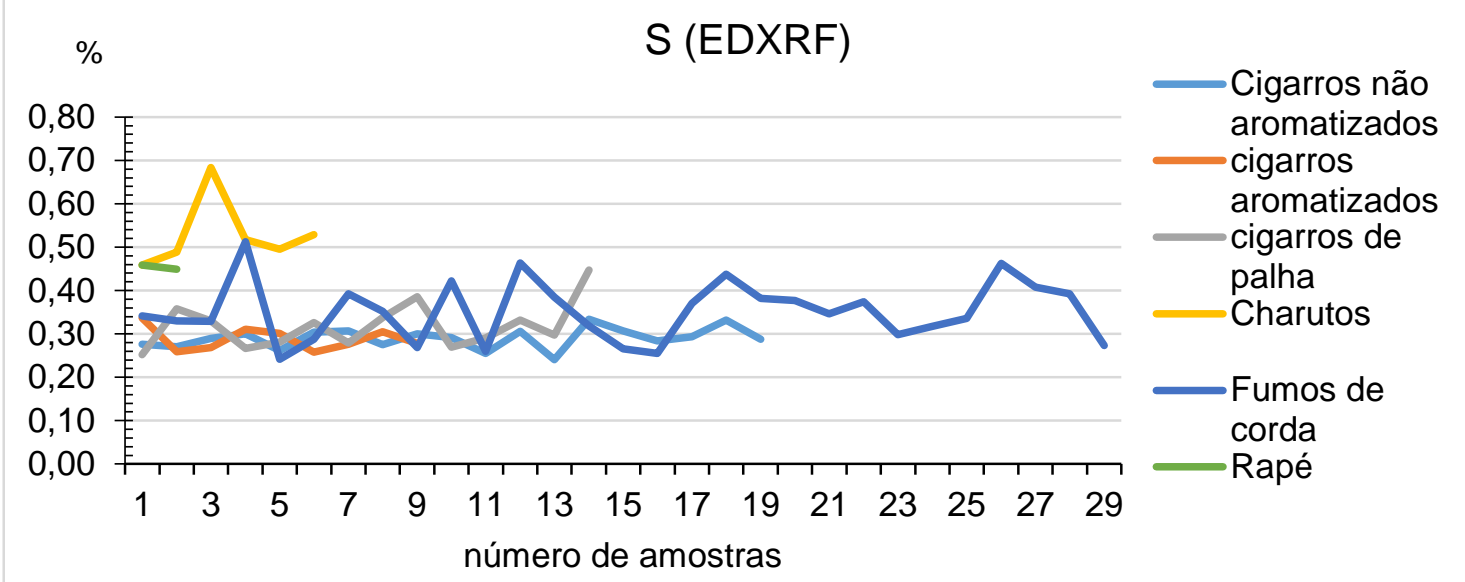

4.7.2.2 Elementos traço As, Br, Cd, Co, Cr, Cs, Cu, $\mathrm{Hg}, \mathrm{Ni}, \mathrm{Pb}, \mathrm{Rb}, \mathrm{Sb}, \mathrm{Se}, \mathrm{Sr}$, Th, U, V, e Zn

$\mathrm{Na}$ TAB. 4.22 são apresentados os resultados das concentrações mínimos, máximos e médios ( $\mathrm{mg} \mathrm{kg}^{-1}$ ) dos elementos $\mathrm{As}, \mathrm{Br}, \mathrm{Cd}, \mathrm{Co}, \mathrm{Cr}, \mathrm{Cs}, \mathrm{Cu}$, $\mathrm{Hg}, \mathrm{Ni}, \mathrm{Pb}, \mathrm{Rb}, \mathrm{Sb}, \mathrm{Se}, \mathrm{Sr}, \mathrm{Th}, \mathrm{U}, \mathrm{V}$ e Zn. 
Tabela 4.22 - Valores médios, mínimos e máximos de concentração dos elementos traço As, $\mathrm{Br}, \mathrm{Cd}, \mathrm{Co}, \mathrm{Cr}, \mathrm{Cs}, \mathrm{Cu}, \mathrm{Hg}, \mathrm{Ni}, \mathrm{Pb}, \mathrm{Rb}$, $\mathrm{Sb}, \mathrm{Se}, \mathrm{Sr}, \mathrm{Th}, \mathrm{U}, \mathrm{V}$ e $\mathrm{Zn}\left(\mathrm{mg} \mathrm{kg}^{-1}\right)$ nos derivados da Nicotiana tabacum L., pela técnica de EDXRF.

\begin{tabular}{|c|c|c|c|c|c|c|c|c|c|c|}
\hline Derivados & & As & $\mathrm{Br}$ & Cd & Co & $\mathrm{Cr}$ & Cs & $\mathrm{Cu}$ & $\mathrm{Hg}$ & $\mathbf{N i}$ \\
\hline \multirow{2}{*}{$\begin{array}{l}\text { Cigarros não } \\
\text { aromatizados }\end{array}$} & Min. & $0,061 \pm 0,002$ & $39 \pm 3$ & $0,19 \pm 0,05$ & $0,45 \pm 0,04$ & $0,81 \pm 0,05$ & $0,041 \pm 0,004$ & $4,4 \pm 0,1$ & $0,024 \pm 0,002$ & $0,72 \pm 0,02$ \\
\hline & Max. & $0,065 \pm 0,001$ & $77 \pm 4$ & $1,17 \pm 0,04$ & $1,20 \pm 0,05$ & $2,5 \pm 0,3$ & $0,050 \pm 0,001$ & $6,0 \pm 0,2$ & $0,034 \pm 0,002$ & $2,2 \pm 0,2$ \\
\hline \multirow{2}{*}{$\begin{array}{c}\text { Cigarros } \\
\text { aromatizados }\end{array}$} & Min. & $0,060 \pm 0,001$ & $38 \pm 5$ & $0,38 \pm 0,04$ & $0,41 \pm 0,01$ & $0,96 \pm 0,05$ & $0,045 \pm 0,002$ & $4,4 \pm 0,1$ & $0,027 \pm 0,001$ & $0,77 \pm 0,03$ \\
\hline & Max. & $0,063 \pm 0,002$ & $74 \pm 9$ & $1,09 \pm 0,05$ & $1,11 \pm 0,05$ & $1,11 \pm 0,05$ & $0,051 \pm 0,001$ & $5,3 \pm 0,1$ & $0,032 \pm 0,001$ & $1,48 \pm 0,03$ \\
\hline \multirow{3}{*}{$\begin{array}{c}\text { Cigarros de } \\
\text { palha }\end{array}$} & Min. & $0,060 \pm 0,003$ & $18,0 \pm 0,5$ & $0,40 \pm 0,03$ & $0,48 \pm 0,01$ & $0,80 \pm 0,03$ & $0,042 \pm 0,002$ & $4,1 \pm 0,2$ & $0,009 \pm 0,004$ & $1,00 \pm 0,05$ \\
\hline & Max. & $0,070 \pm 0,002$ & $75 \pm 3$ & $2,7 \pm 0,3$ & $1,28 \pm 0,21$ & $1,83 \pm 0,05$ & $0,065 \pm 0,001$ & $6,0 \pm 0,1$ & $0,035 \pm 0,001$ & $2,72 \pm 0,21$ \\
\hline & Med. & $0,062 \pm 0,003$ & $41 \pm 18$ & $1,31 \pm 0,74$ & $0,77 \pm 0,27$ & $1,33 \pm 0,38$ & $0,054 \pm 0,008$ & $5,0 \pm 0,5$ & $0,027 \pm 0,007$ & $1,47 \pm 0,52$ \\
\hline \multirow{3}{*}{ Charutos } & Min. & $0,061 \pm 0,002$ & $38 \pm 2$ & $0,38 \pm 0,02$ & $0,33 \pm 0,03$ & $0,89 \pm 0,04$ & $0,046 \pm 0,001$ & $4,91 \pm 0,04$ & $0,024 \pm 0,001$ & $0,92 \pm 0,05$ \\
\hline & Max. & $0,067 \pm 0,004$ & $98 \pm 5$ & $1,30 \pm 0,5$ & $0,84 \pm 0,05$ & $1,30 \pm 0,04$ & $0,054 \pm 0,001$ & $6,20 \pm 0,05$ & $0,032 \pm 0,001$ & $1,42 \pm 0,02$ \\
\hline & Med. & $0,064 \pm 0,002$ & $84 \pm 23$ & $0,74 \pm 0,35$ & $0,50 \pm 0,18$ & $1,04 \pm 0,18$ & $0,049 \pm 0,003$ & $5,45 \pm 0,47$ & $0,027 \pm 0,003$ & $1,13 \pm 0,16$ \\
\hline \multirow{2}{*}{$\begin{array}{l}\text { Fumos de } \\
\text { corda }\end{array}$} & Max. & $0,070 \pm 0,003$ & $257 \pm 6$ & $4,0 \pm 0,2$ & $1,36 \pm 0,04$ & $1,2 \pm 0,1$ & $0,057 \pm 0,001$ & $7,9 \pm 0,3$ & $1,3 \pm 0,2$ & $1,9 \pm 0,4$ \\
\hline & Med. & $0,063 \pm 0,002$ & $69 \pm 60$ & $2,4 \pm 0,6$ & $0,71 \pm 0,28$ & $0,96 \pm 0,09$ & $0,045 \pm 0,0092$ & $5,7 \pm 0,4$ & $0,030 \pm 0,005$ & $1,27 \pm 0,35$ \\
\hline \multirow{3}{*}{ Rapés } & Min. & $0,062 \pm 0,001$ & $70 \pm 5$ & $3,39 \pm 0,07$ & $1,27 \pm 0,02$ & $0,88 \pm 0,02$ & $0,049 \pm 0,001$ & $5,75 \pm 0,05$ & $0,028 \pm 0,001$ & $<0,61$ \\
\hline & Max. & $0,062 \pm 0,001$ & $75 \pm 3$ & $3,75 \pm 0,05$ & $1,36 \pm 0,02$ & $0,90 \pm 0,04$ & $0,054 \pm 0,001$ & $5,39 \pm 0,2$ & $0,031 \pm 0,001$ & $2,21 \pm 0,03$ \\
\hline & Med. & $0,062 \pm 0,001$ & $72 \pm 4$ & $3,57 \pm 0,25$ & $1,31 \pm 0,06$ & $0,89 \pm 0,015$ & $0,052 \pm 0,003$ & $5,57 \pm 0,26$ & $0,029 \pm 0,002$ & - \\
\hline
\end{tabular}

- não determinado 
Continuação da Tab. 4.22 - Valores médios mínimos e máximos de concentração do elemento traço As, Br, Cd, Co, Cr, Cs, Cu, Hg, $\mathrm{Ni}, \mathrm{Pb}, \mathrm{Rb}, \mathrm{Sb}, \mathrm{Se}, \mathrm{Sr}, \mathrm{Th}, \mathrm{U}, \mathrm{V}$ e Zn $\left(\mathrm{mg} \mathrm{kg}^{-1}\right)$ nos derivados da Nicotiana tabacum L., pela técnica de EDXRF.

\begin{tabular}{|c|c|c|c|c|c|c|c|c|c|c|}
\hline Derivado & & $\mathrm{Pb}$ & $\mathbf{R b}$ & $\mathrm{Sb}$ & $\mathrm{Se}$ & $\mathrm{Sr}$ & Th & $\mathbf{U}$ & v & $\mathrm{Zn}$ \\
\hline \multirow{3}{*}{$\begin{array}{l}\text { Cigarros não } \\
\text { aromatizados }\end{array}$} & Min. & $0,26 \pm 0,03$ & $19,4 \pm 0,1$ & $0,018 \pm 0,001$ & $0,039 \pm 0,001$ & $80 \pm 1$ & $0,016 \pm 0,001$ & $0,10 \pm 0 ; 02$ & $0,76 \pm 0,04$ & $15,9 \pm 0,2$ \\
\hline & Max. & $1,48 \pm 0,03$ & $20,8 \pm 0,4$ & $0,026 \pm 0,001$ & $0,045 \pm 0,001$ & $131 \pm 1$ & $0,097 \pm 0,033$ & $0,19 \pm 0,03$ & $2,1 \pm 0,3$ & $22,8 \pm 0,2$ \\
\hline & Med. & $0,97 \pm 0,44$ & $19,8 \pm 0,3$ & $0,022 \pm 0,002$ & $0,043 \pm 0,002$ & $100 \pm 15$ & $0,056 \pm 0,026$ & $0,16 \pm 0,02$ & $1,26 \pm 0,39$ & $18,1 \pm 2,1$ \\
\hline \multirow{3}{*}{$\begin{array}{c}\text { Cigarros } \\
\text { aromatizados }\end{array}$} & Min. & $0,44 \pm 0,05$ & $19,4 \pm 0,2$ & $0,021 \pm 0,001$ & $0,042 \pm 0,001$ & $88 \pm 1$ & $0,050 \pm 0,001$ & $0,15 \pm 0,05$ & $0,69 \pm 0,04$ & $9,4 \pm 0,2$ \\
\hline & Max. & $1,73 \pm 0,04$ & $20,1 \pm 0,3$ & $0,024 \pm 0,001$ & $0,044 \pm 0,001$ & $128 \pm 1$ & $0,078 \pm 0,001$ & $0,25 \pm 0,03$ & $1,70 \pm 0,04$ & $20,2 \pm 0,3$ \\
\hline & Med. & $1,06 \pm 0,46$ & $19,7 \pm 0,2$ & $0,023 \pm 0,001$ & $0,043 \pm 0,001$ & $107 \pm 15$ & $0,064 \pm 0,010$ & $0,19 \pm 0,04$ & $1,35 \pm 0,34$ & $18,0 \pm 3,3$ \\
\hline \multirow{3}{*}{$\begin{array}{c}\text { Cigarros de } \\
\text { palha }\end{array}$} & Min. & $0,40 \pm 0,004$ & $15,3 \pm 0,2$ & $0,019 \pm 0,001$ & $0,041 \pm 0,001$ & $64,1 \pm 0,3$ & $0,047 \pm 0,002$ & $0,13 \pm 0,03$ & $0,70 \pm 0,03$ & $17,2 \pm 0,2$ \\
\hline & Max. & $1,8 \pm 0,03$ & $21 \pm 1$ & $0,043 \pm 0,004$ & $0,047 \pm 0,001$ & $181 \pm 1$ & $0,122 \pm 0,001$ & $0,33 \pm 0,02$ & $3,72 \pm 0,05$ & $24,8 \pm 0,3$ \\
\hline & Med. & $1,08 \pm 0,53$ & $18 \pm 2$ & $0,025 \pm 0,006$ & $0,044 \pm 0,002$ & $94 \pm 30$ & $0,070 \pm 0,025$ & $0,21 \pm 0,06$ & $1,70 \pm 0,92$ & $21,0 \pm 2,4$ \\
\hline \multirow{3}{*}{ Charutos } & Min. & $0,44 \pm 0,05$ & $18,2 \pm 0,2$ & $0,022 \pm 0,001$ & $0,043 \pm 0,001$ & $113 \pm 1$ & $0,045 \pm 0,001$ & $0,13 \pm 0,02$ & $0,72 \pm 0,04$ & $21,1 \pm 0,2$ \\
\hline & Max. & $1,84 \pm 0,05$ & $20,4 \pm 0,4$ & $0,026 \pm 0,001$ & $0,045 \pm 0,001$ & $167 \pm 1$ & $0,076 \pm 0,001$ & $0,27 \pm 0,05$ & $1,11 \pm 0,04$ & $24,6 \pm 0,3$ \\
\hline & Med. & $1,08 \pm 0,46$ & $19,6 \pm 1,0$ & $0,024 \pm 0,002$ & $0,044 \pm 0,001$ & $144 \pm 21$ & $0,059 \pm 0,011$ & $0,20 \pm 0,05$ & $1,01 \pm 0,14$ & $22,1 \pm 1,3$ \\
\hline \multirow{3}{*}{$\begin{array}{c}\text { Fumos de } \\
\text { corda }\end{array}$} & Min. & $0,8 \pm 0,1$ & $18,8 \pm 0,4$ & $0,0228 \pm 0,0004$ & $0,043 \pm 0,001$ & $64,5 \pm 0,4$ & $0,037 \pm 0,001$ & $0,15 \pm 0,02$ & $0,40 \pm 0,05$ & $17,5 \pm 0,3$ \\
\hline & Max. & $1,6 \pm 0,4$ & $20,8 \pm 0,3$ & $0,033 \pm 0,003$ & $0,048 \pm 0,001$ & $187,5 \pm 0,3$ & $0,133 \pm 0,002$ & $0,37 \pm 0,01$ & $2,4 \pm 0,3$ & $27,7 \pm 0,3$ \\
\hline & Med. & $1,0 \pm 0,4$ & $20,1 \pm 0,54$ & $0,028 \pm 0,002$ & $0,044 \pm 0,008$ & $111,1 \pm 33,5$ & $0,095 \pm 0,024$ & $0,24 \pm 0,06$ & $1,23 \pm 0,48$ & $22,6 \pm 2,7$ \\
\hline \multirow{3}{*}{ Rapés } & Min. & $0,83 \pm 0,02$ & $20,4 \pm 0,3$ & $0,032 \pm 0,001$ & $0,049 \pm 0,001$ & $108 \pm 1$ & $0,11 \pm 0,01$ & $0,26 \pm 0,04$ & $1,9 \pm 0,2$ & $19,7 \pm 0,2$ \\
\hline & Max. & $1,45 \pm 0,04$ & $20,5 \pm 0,2$ & $0,035 \pm 0,001$ & $0,049 \pm 0,001$ & $110 \pm 1$ & $0,12 \pm 0,01$ & $0,34 \pm 0,01$ & $3,65 \pm 0,2$ & $23,5 \pm 0,5$ \\
\hline & Med. & $1,14 \pm 0,44$ & $20,4 \pm 0,1$ & $0,034 \pm 0,002$ & $0,049 \pm 0,001$ & $110 \pm 2$ & $0,12 \pm 0,01$ & $0,30 \pm 0,06$ & $2,80 \pm 1,20$ & $21,6 \pm 2,7$ \\
\hline
\end{tabular}


PAPPAS (2011) analisando cigarros de diferentes nacionalidades determinou concentrações de As em cigarros canadenses de $0,151 \mu \mathrm{g} \mathrm{g}^{-1}$, cigarros indianos de $0,73 \mu \mathrm{g} \mathrm{g}^{-1}$ a $0,86 \mu \mathrm{g} \mathrm{g}^{-1}$ e $0,25 \mu \mathrm{g} \mathrm{g}^{-1}$ em cigarros americanos. PIEDÉ (2015) determinou a concentração média do elemento As de $237 \mathrm{ng} \mathrm{g}^{-1} \mathrm{em}$ cigarros de diversos países. ELDRIDGE et al. (2015) analisaram o conteúdo de As em filtros de cigarro e determinaram a concentração média de 0,211 $\mu \mathrm{g} \mathrm{g}^{-1}$. Os resultados encontrados no presente trabalho, variação de $0,060 \mathrm{\mu g} \mathrm{g}^{-1}$ nas amostras de cigarro de palha CPCPC e cigarro aromatizado CAMFM a 0,070 $\pm 0,002 \mu \mathrm{g} \mathrm{g}^{-1}$ na amostra de cigarro de palha CPTV, estão abaixo dos encontrados pelos referidos autores. A FIG. 4.35 apresenta a variação da concentração do elemento As no grupo amostral, os grupos que apresentaram maiores variações de concentração foram os de cigarro de palh, charutos e fumo de corda.

FIGURA 4.35 - Variação da concentração do elemento $P\left(\mathrm{mg} \mathrm{kg}^{-1}\right)$ nos derivados da Nicotiana tabacum L. pela técnica EDXRF.

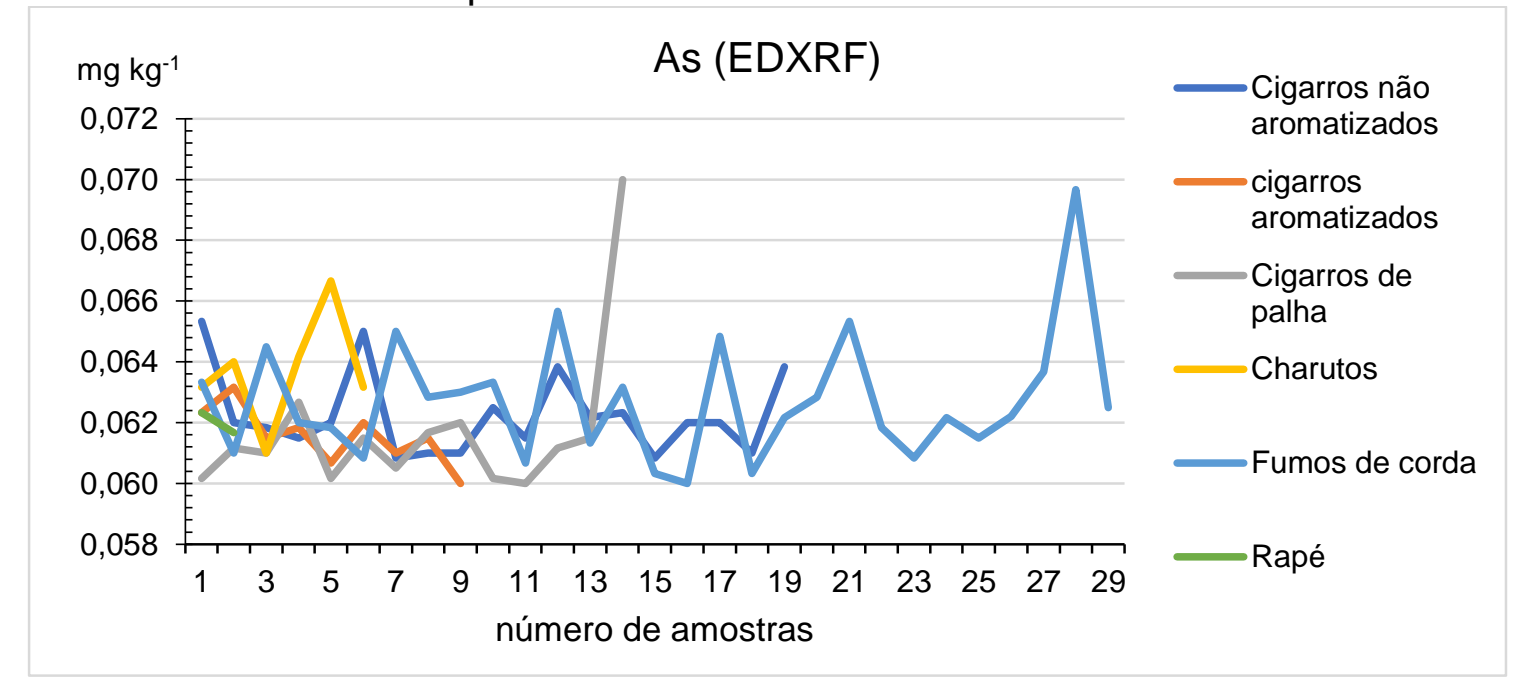

As concentrações determinadas para o elemento $\mathrm{Br}$ determinadas nos cigarros analisados no presente trabalho, variação de $38 \mathrm{mg} \mathrm{kg}^{-1}$ a $77 \mathrm{mg} \mathrm{kg}^{-1}$, estão de acordo com as obtidas por MÜLLER et al. (2011) que analisaram amostras de cigarros brasileiros, onde obtiveram concentrações que variaram de $29,8 \mu \mathrm{g} \mathrm{g}^{-1}$ a 53,3 $\mu \mathrm{g} \mathrm{g}^{-1}$. Os autores citam que a concentração de $\mathrm{Br}$ atingir valores de até 200

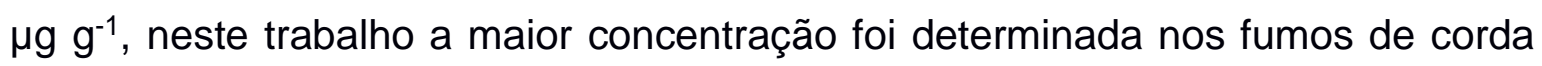


FCA4, com concentração máxima de $257 \mathrm{mg} \mathrm{kg}^{-1}$. Na FIG 4.36 observa-se a variação do $\mathrm{Br}$ em todo o grupo amostral.

FIGURA 4.36 - Variação da concentração do elemento $\mathrm{Br}\left(\mathrm{mg} \mathrm{kg}^{-1}\right)$ nos derivados da Nicotiana tabacum L. pela técnica EDXRF.

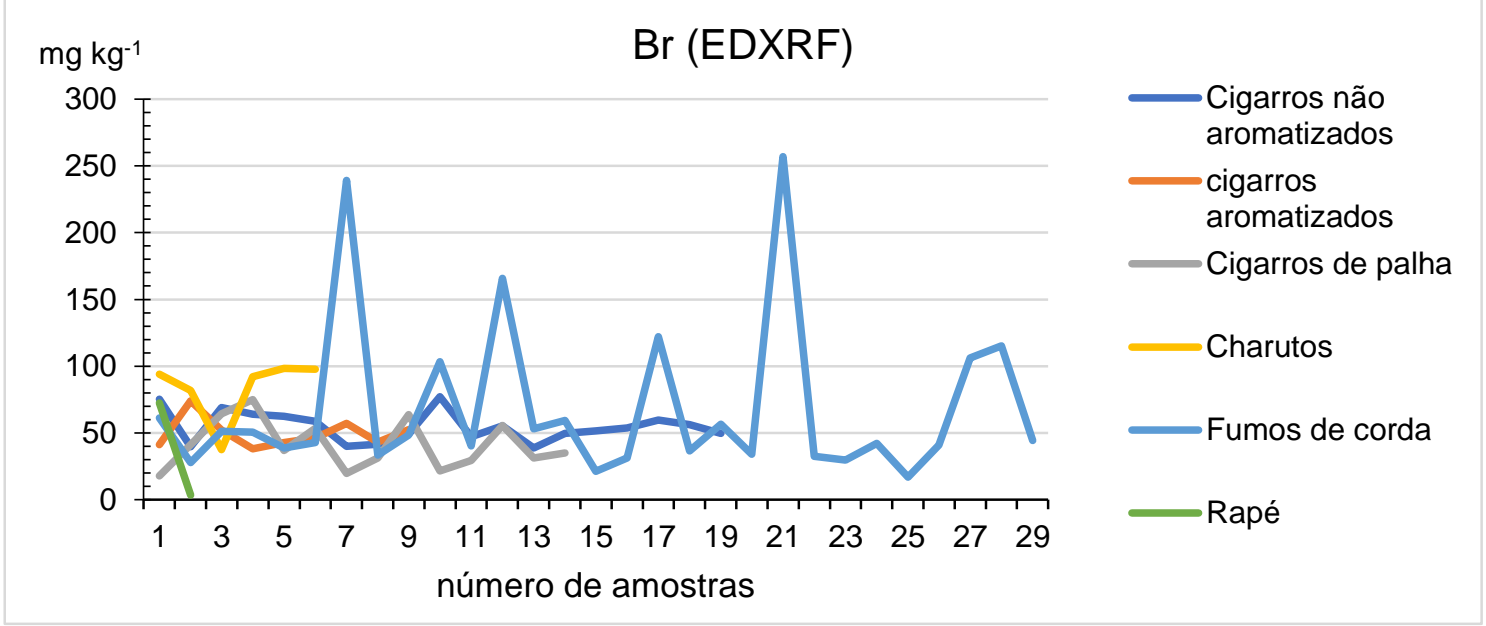

O elemento $\mathrm{Cd}$ apresentou valores de concentrações que variaram de 0,30 \pm 0,05 $\mu \mathrm{g} \mathrm{g}^{-1}$ na amostra de cigarro não aromatizado CCDV a 4,0 \pm 0,2 $\mu \mathrm{g} \mathrm{g}^{-1}$ na amostra de fumo de corda FCA10; estes valores estão na mesma faixa de concentração dos autores: PAPPAS (2011) que obteve variação na concentração de $0,28 \mu \mathrm{g} \mathrm{g}^{-1} \mathrm{a} 4,5 \mu \mathrm{g} \mathrm{g}^{-1}$, KAZI et al (2009) que analisou filtros de cigarro e obteve

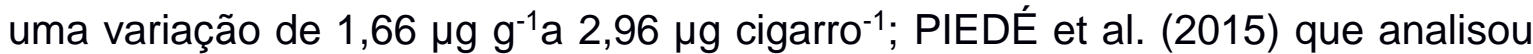
filtros de cigarros e obteve uma concentração média de $898 \mathrm{ng} \mathrm{g}^{-1}$ e ELDRIDGE et al. (2015) que analisou filtros de cigarros e obteve uma concentração média de $0,988 \mu \mathrm{g} \mathrm{g}^{-1}$. A FIG. 4.37 apresenta a variação da concentração nos grupos de amostras. 
FIGURA 4.37 - Variação da concentração do elemento $\mathrm{Cd}\left(\mathrm{mg} \mathrm{kg}^{-1}\right)$ nos derivados da Nicotiana tabacum L. pela técnica EDXRF.

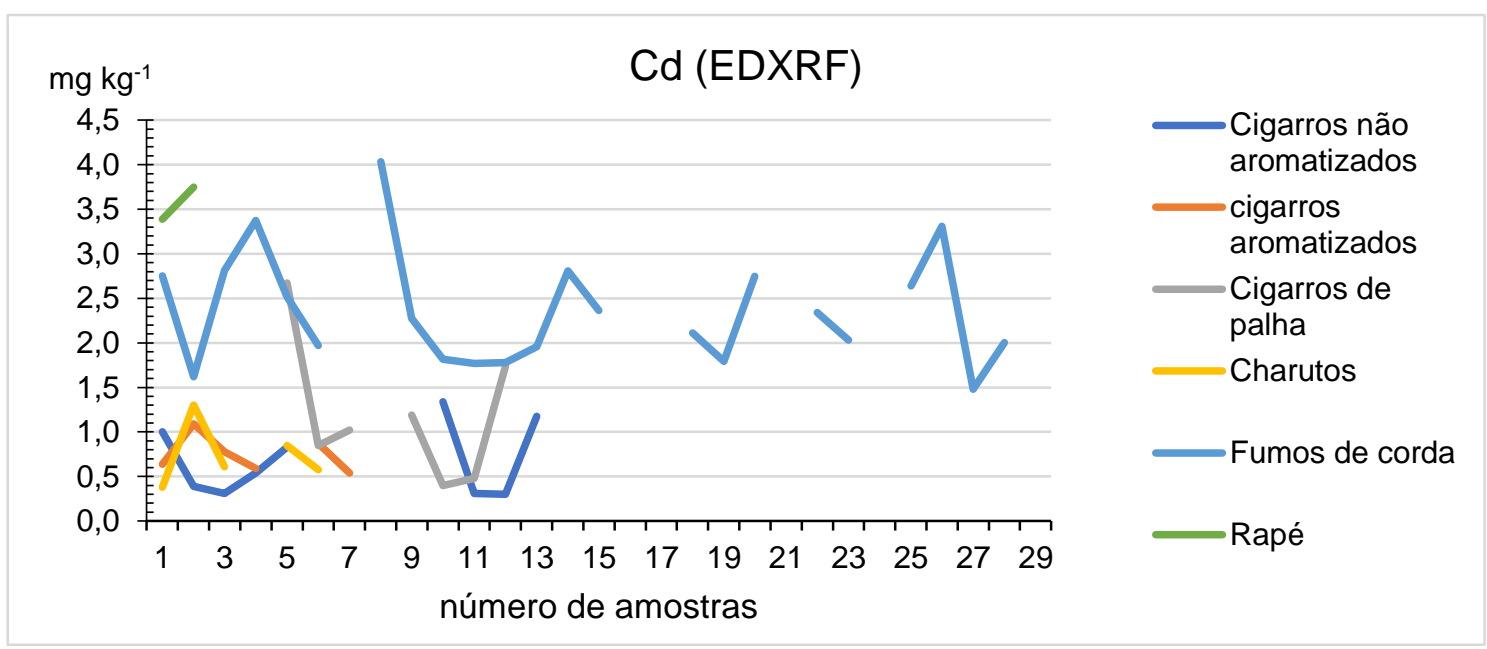

Os valores obtidos de concentração para o elemento Co variaram de 0,33 \pm 0,03 $\mu \mathrm{g} \mathrm{g}^{-1}$ na amostra de charuto CHMPE a 1,36 $\pm 0,02 \mu \mathrm{g} \mathrm{g}^{-1}$ na amostra de rapé RPCM e estão de acordo com os valores obtidos por PAPPAS (2011), cuja variação foi de $0,01 \mu \mathrm{g} \mathrm{g}^{-1}$ a 0,425 $\mu \mathrm{g} \mathrm{g}^{-1}$ em cigarros. A FIG. 4.38 apresenta a variação de concentração do elemento no grupo de amostras. Pode-se observar uma grande variação nos resultados para todas as amostras analisadas, com exceção para as duas amostras de rapé.

FIGURA 4.38 - Variação da concentração do elemento Co $\left(\mathrm{mg} \mathrm{kg}^{-1}\right)$ nos derivados da Nicotiana tabacum L. pela técnica EDXRF.

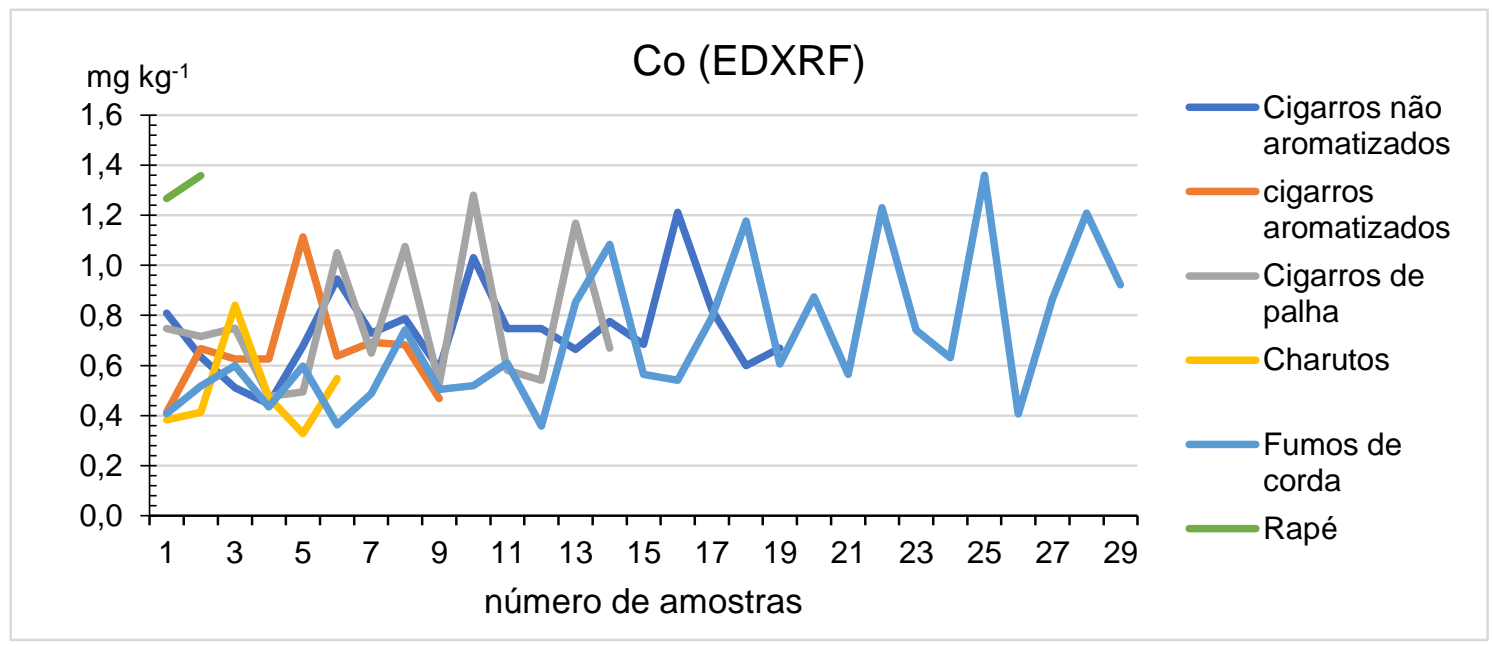


Os valores obtidos de concentração para o elemento $\mathrm{Cr}$ variaram $0,80 \pm$ 0,03 $\mu \mathrm{g} \mathrm{g}^{-1}$ na amostra de cigarro de palha CPTV a 2,5 $\pm 0,3 \mu \mathrm{g} \mathrm{g}^{-1}$ na amostra de cigarro não aromatizado CCDA, valores concordantes com a variação de concentração obtida por PAPPAS (2011) de 0,353 $\mu \mathrm{g} \mathrm{g}^{-1}$ a 5,0 $\mu \mathrm{g} \mathrm{g}^{-1}$ determinada em cigarros. A FIG. 4.39 apresenta a variação de concentração do elemento no grupo de amostras; as maiores variações foram obtidas para o grupo de cigarro de palha.

FIGURA 4.39 - Variação da concentração do elemento $\mathrm{Cr}\left(\mathrm{mg} \mathrm{kg}^{-1}\right)$ nos derivados da Nicotiana tabacum L. pela técnica EDXRF.

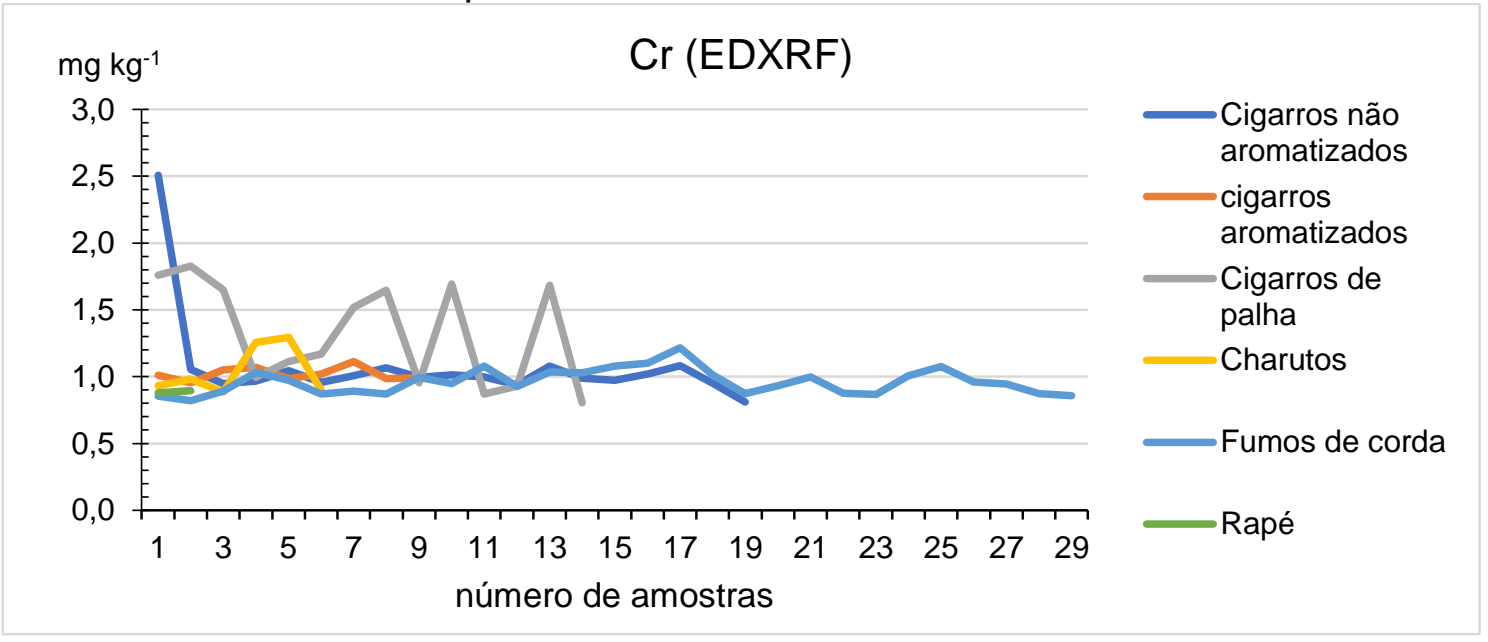

A concentração do elemento Cs variou de 0,040 \pm 0,001 \% na amostra de fumo de corda FCA4 a 0,065 \pm 0,001 \% na amostra de cigarro de palha CPVC. A FIG. 4.40 apresenta a variação da concentração do elemento Cs nos grupos de amostras; o grupo que apresentou as maiores concentrações foi o cigarro de palha. 
FIGURA 4.40 - Variação da concentração do elemento Cs $\left(\mathrm{mg} \mathrm{kg}^{-1}\right)$ nos derivados da Nicotiana tabacum L. pela técnica EDXRF.

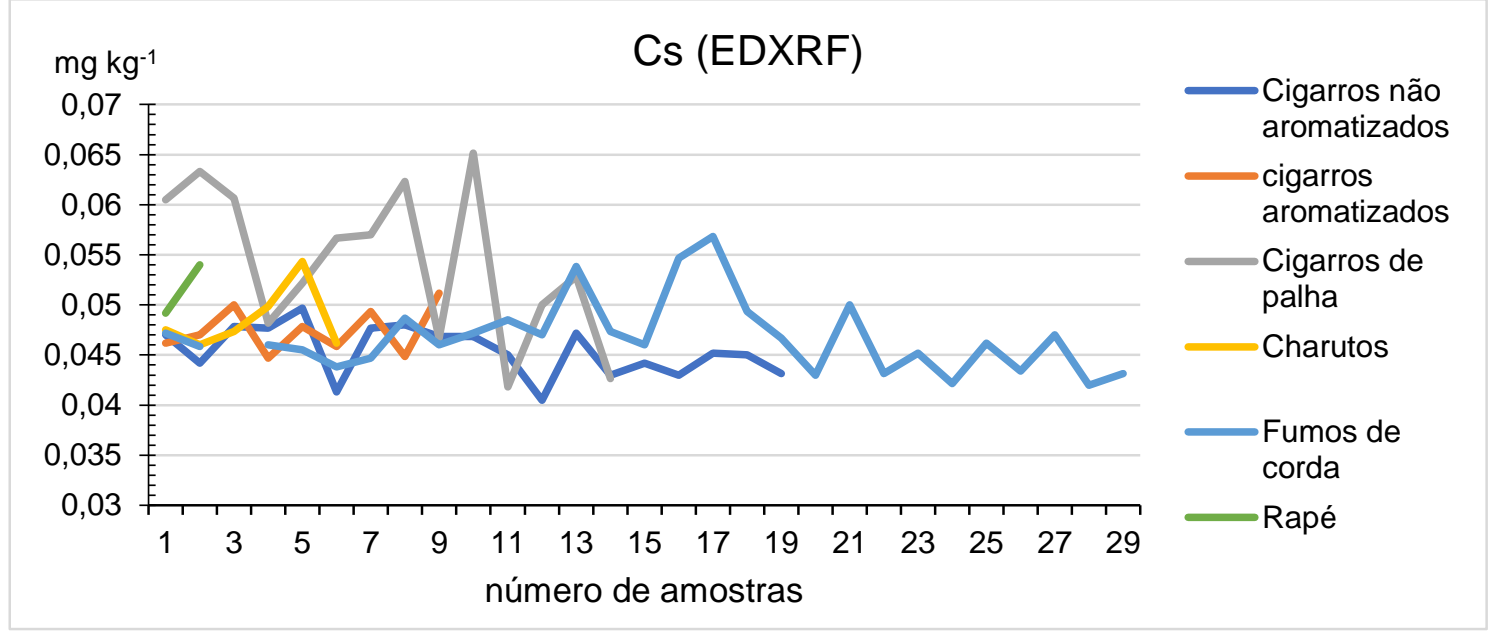

O elemento $\mathrm{Cu}$ também apresentou concentrações de acordo com PAPPAS (2015). O autor reporta variação das concentrações de 3,49 a 19,2 $\mu \mathrm{g} \mathrm{g}^{-1}$ e neste trabalho a variação foi de $4,1 \pm 0,2 \mu \mathrm{g} \mathrm{g}^{-1}$ a 7,9 $\pm 0,3 \mu \mathrm{g} \mathrm{g}^{-1}$. A FIG. 4.41 apresenta a variação da concentração do elemento Cu nos grupos de amostras.

FIGURA 4.41 - Variação da concentração do elemento Cu $\left(\mathrm{mg} \mathrm{kg}^{-1}\right)$ nos derivados da Nicotiana tabacum L. pela técnica EDXRF.

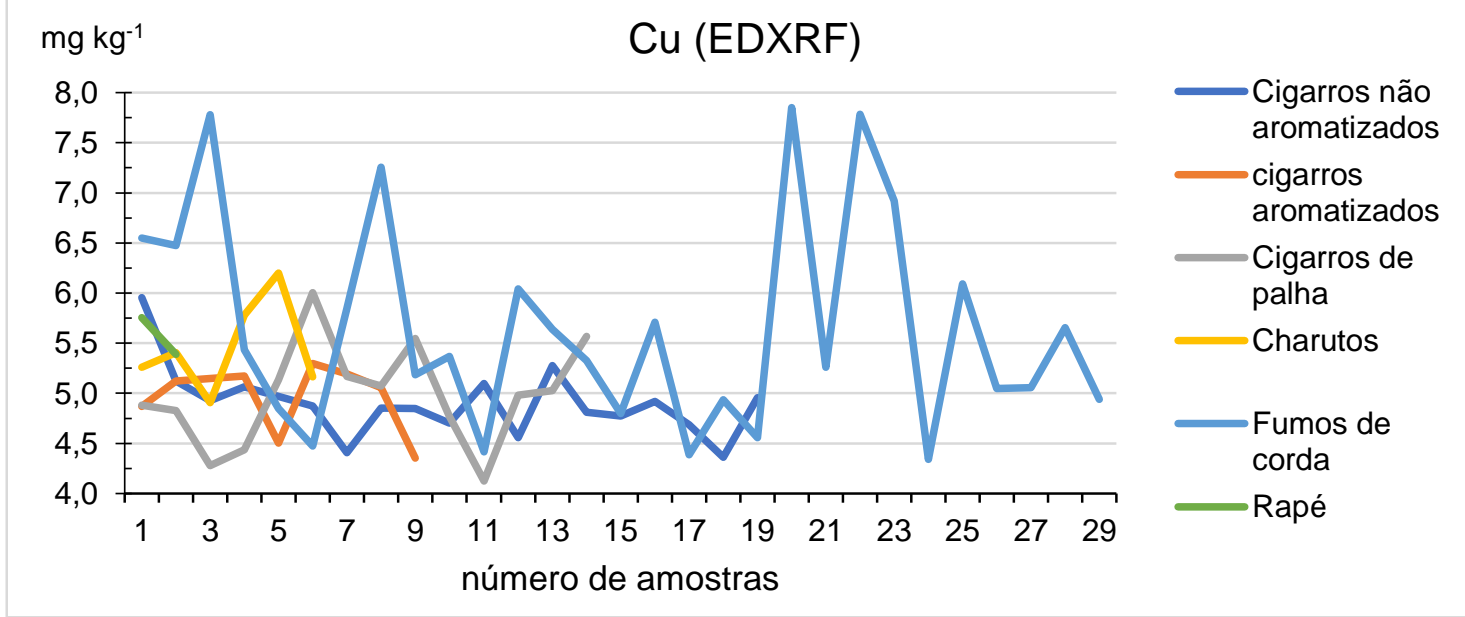

Para o elemento Hg, o autor PAPPAS (2011) reporta concentrações de $0,020 \mu \mathrm{g} \mathrm{g}^{-1}$ a $0,027 \mu \mathrm{g} \mathrm{g}^{-1}$ em cigarros, os valores médios obtidos no presente trabalho variaram de 0,019 $\pm 0,004 \mu \mathrm{g} \mathrm{g}^{-1}$ na amostra de fumo de corda FCA4 a 0,040 \pm 0,001 $\mu \mathrm{g} \mathrm{g}^{-1}$ na amostra de fumo de corda FCDTT. A FIG. 4.42 apresenta a variação da concentração do Hg nos grupos de amostras. 
FIGURA 4.42 - Variação da concentração do elemento $\mathrm{Hg}\left(\mathrm{mg} \mathrm{kg}^{-1}\right)$ nos derivados da Nicotiana tabacum L. pela técnica EDXRF.

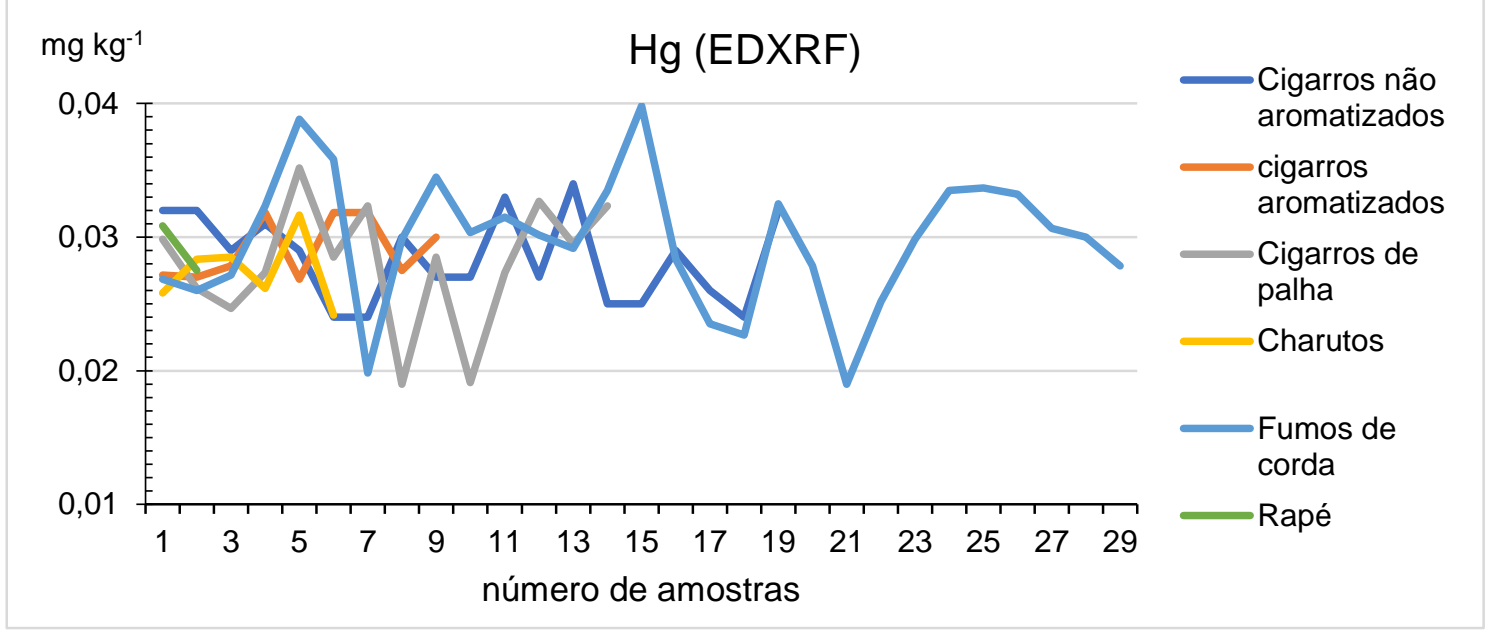

As concentrações do elemento $\mathrm{Ni}$ foi determinada na maioria das amostras e os valores obtidos variaram de 0,61 $\pm 0,05 \mu \mathrm{g} \mathrm{g}^{-1}$ na amostra de fumo de corda FCSC a 2,7 $\pm 0,2 \mu \mathrm{g} \mathrm{g}^{-1}$ na amostra de cigarro de palha CPSP, estes valores estão dentro da faixa de concentração reportada por PAPPAS (2011) que obteve um intervalo de concentração de 0,25 $\mathrm{g} \mathrm{g} \mathrm{g}^{-1}$ a 10,2 $\mu \mathrm{g} \mathrm{g}^{-1}$. KAZI et al (2009) obteve variação de 0,725 a 1,34 $\mu \mathrm{g} \mathrm{g}^{-1}$ e ELDRIDGE (2015) valores médios de $2,046 \mu \mathrm{g} \mathrm{g}^{-1}$ em análises de filtros de cigarros. A FIG. 4.43 apresenta a variação do elemento Ni nos grupos de amostras.

FIGURA 4.43 - Variação da concentração do elemento $\mathrm{Hg}\left(\mathrm{mg} \mathrm{kg}^{-1}\right)$ nos derivados da Nicotiana tabacum L. pela técnica EDXRF.

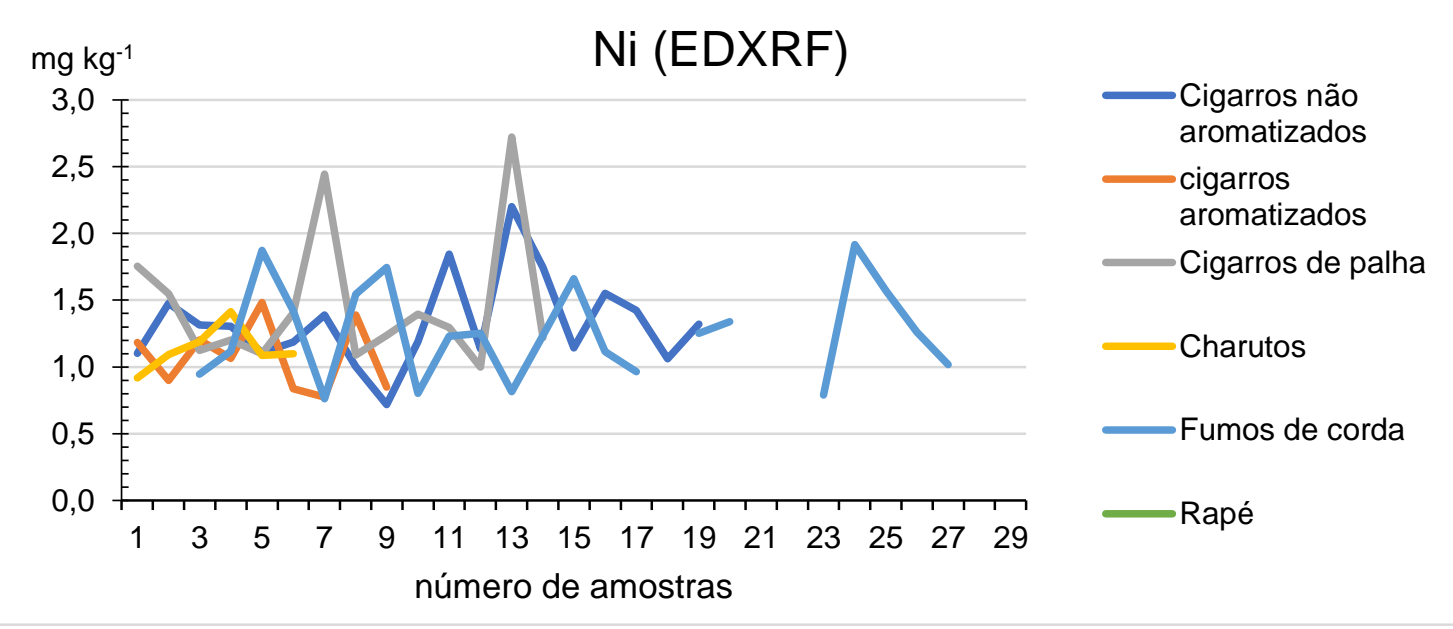


Os valores de concentração para o elemento $\mathrm{Pb}$ são da mesma ordem de grandeza dos obtidos nas análises de filtros de cigarro dos autores KAZI et al. (2009), ELDRIGE et al. (2015) e PIEDÉ et al. (2015), cujos valores variaram de $0,399 \mu \mathrm{g} \mathrm{g}^{-1}-1,39 \mu \mathrm{g} \mathrm{g^{-1 }}$ para o primeiro autores e valores médios de 0,620 $\mu \mathrm{g} \mathrm{g}^{-1} \mathrm{e}$ $466 \mathrm{ng} \mathrm{\textrm {g } ^ { - 1 }}$, para o segundo e terceiro autor, respectivamente Os valores determinados no presente trabalho variaram de 0,26 $\pm 0,03 \mu \mathrm{g} \mathrm{g}^{-1}$ na amostra de cigarro não aromatizado CCFV a 1,84 $\pm 0,05 \mu \mathrm{g} \mathrm{g}^{-1}$ na amostra de charuto CHDNE. A FIG. 4.44 apresenta a variação da concentração do elemento $\mathrm{Pb}$ nas amostras analisadas.

FIGURA 4.44 - Variação da concentração do elemento $\mathrm{Hg}\left(\mathrm{mg} \mathrm{kg}^{-1}\right)$ nos derivados da Nicotiana tabacum L. pela técnica EDXRF.

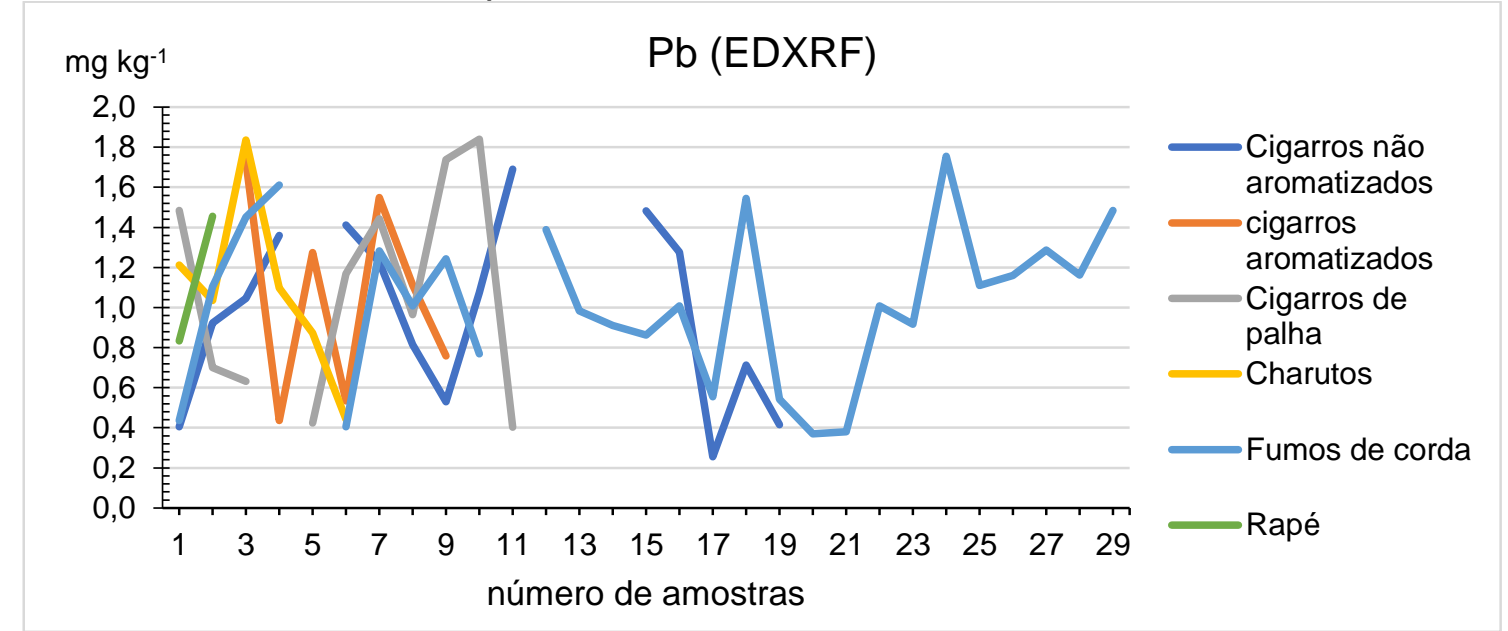

A concentração do elemento $\mathrm{Rb}$ variou de 15,3 \pm 0,2 $\mathrm{mg} \mathrm{kg}^{-1}$ na amostra de cigarro de palha CPC a $21 \pm 1 \mathrm{mg} \mathrm{kg}^{-1}$ na amostra de cigarro de palha CPTV. A FIG. 4.45 apresenta a variação da concentração do elemento $\mathrm{Rb}$ nos grupos amostrados; o grupo que apresentou as maiores concentrações foi o cigarro de palha. 
FIGURA 4.45 - Variação da concentração do elemento Rb $\left(\mathrm{mg} \mathrm{kg}^{-1}\right)$ nos derivados da Nicotiana tabacum L. pela técnica EDXRF.

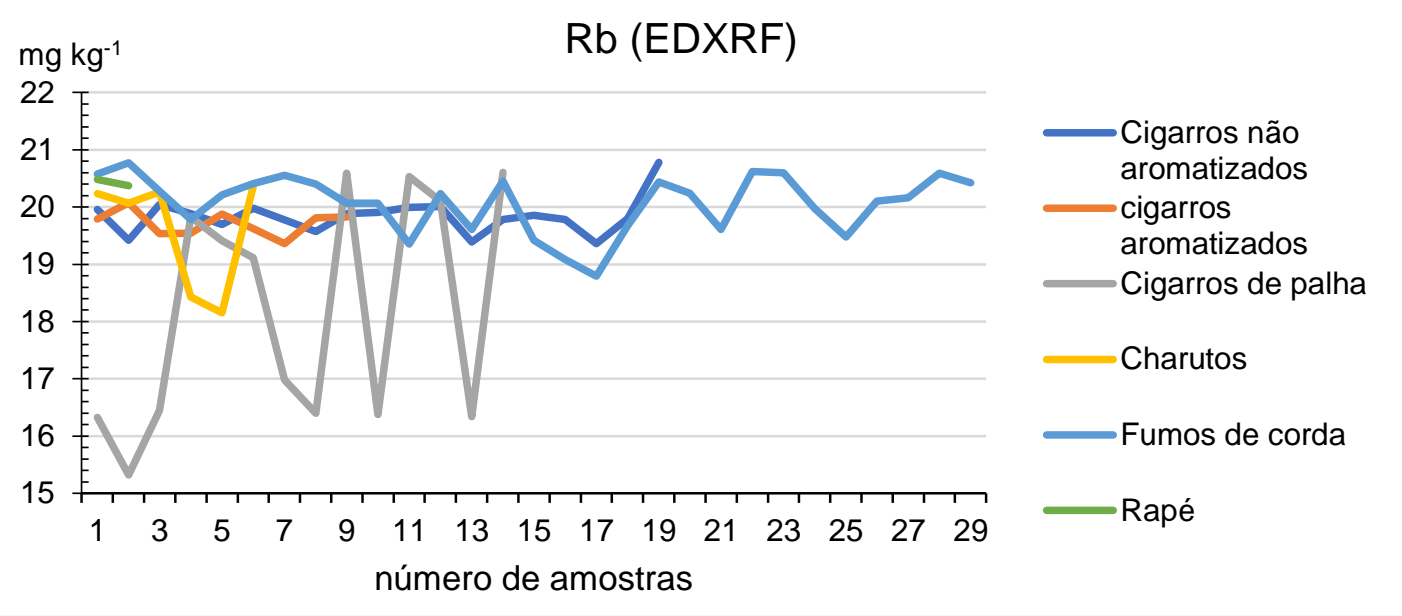

A concentração do elemento Sb variou de 0,018 $\pm 0,001 \mathrm{mg} \mathrm{kg}^{-1} \mathrm{na}$ amostra de cigarro não aromatizado CCSH a 0,043 \pm 0,004 $\mathrm{mg} \mathrm{kg}^{-1}$ na amostra de cigarro de palha CPTV. A FIG. 4.46 apresenta a variação da concentração do elemento $\mathrm{Sb}$ nos grupos amostrados; o grupo que apresentou as maiores concentrações foi o cigarro de palha.

FIGURA 4.46 - Variação da concentração do elemento Sb $\left(\mathrm{mg} \mathrm{kg}^{-1}\right)$ nos derivados da Nicotiana tabacum L. pela técnica EDXRF.

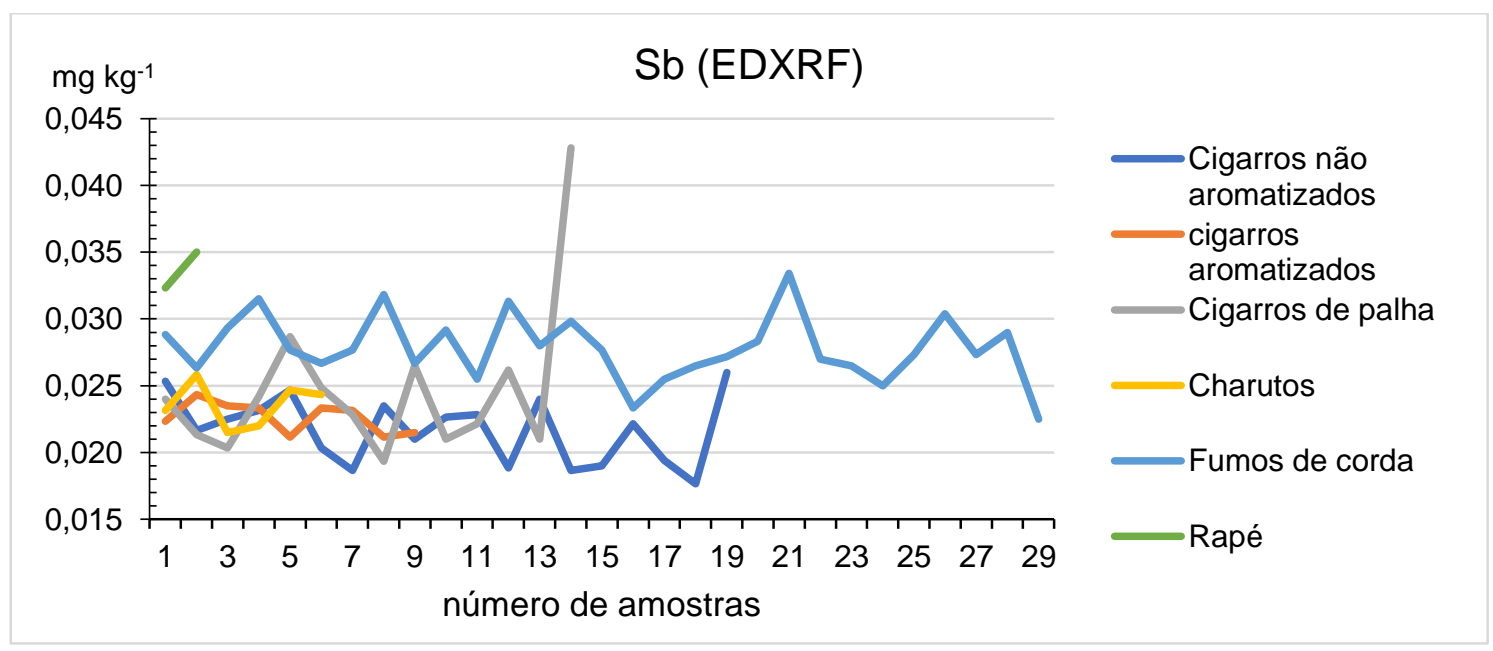

A concentração do elemento Se variou de 0,039 $\pm 0,001 \mathrm{mg} \mathrm{kg}^{-1}$ na amostra de cigarro não aromatizado CCSH a 0,049 $\pm 0,004 \mathrm{mg} \mathrm{kg}^{-1}$ na amostra de rapé RPCM. ELDRIDGE et al. (2015) obteve valores médios de concentração de Se de $0,13 \mu \mathrm{g} \mathrm{g}^{-1}$ em filtros de cigarros, valores muito superiores aos determinados no presente trabalho. A FIG. 4.47 apresenta a variação da concentração do 
elemento Se nos grupos amostrados; os grupos de amostras que apresentaram maiores variações foram os de cigarro não aromatizado, cigarro de palha, charuto e fumo de corda.

FIGURA 4.47- Variação da concentração do elemento Se $\left(\mathrm{mg} \mathrm{kg}^{-1}\right)$ nos derivados da Nicotiana tabacum L. pela técnica EDXRF.

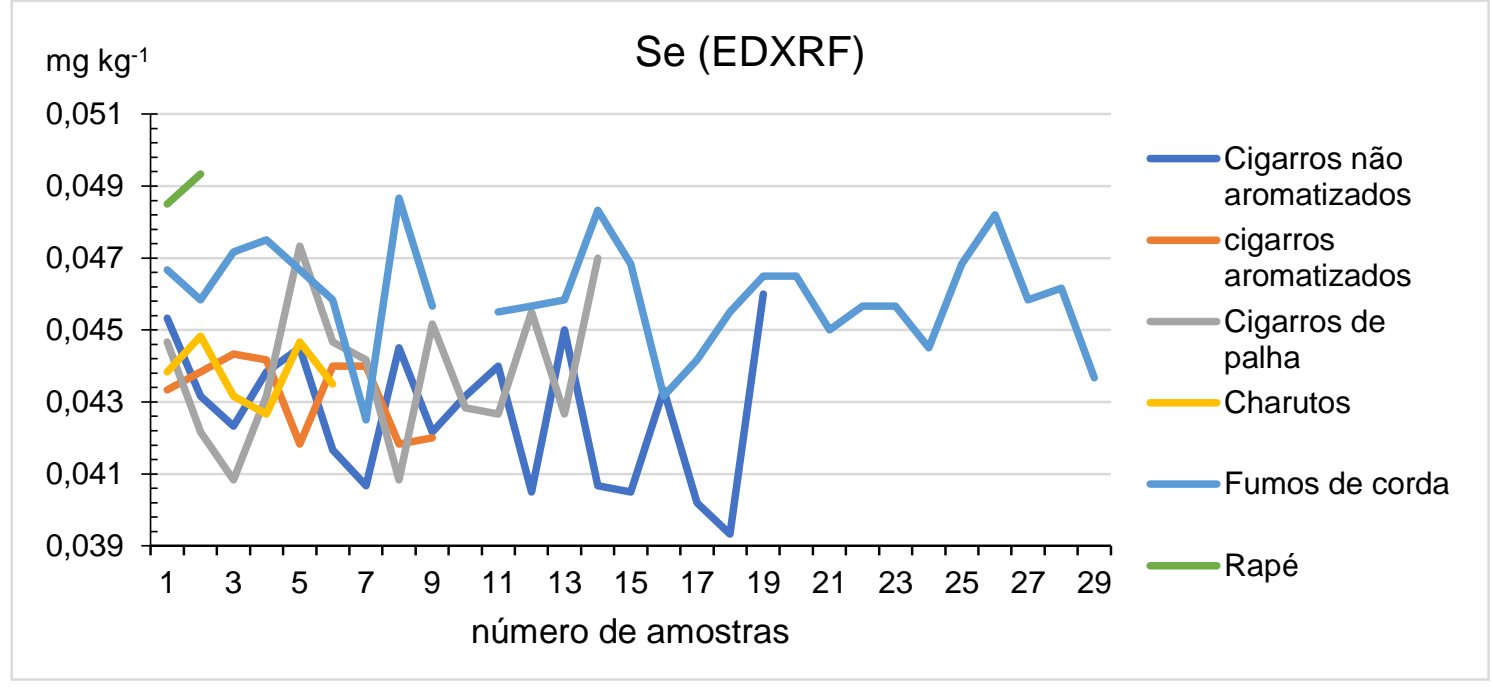

A concentração do elemento Sr variou de $64,1 \pm 0,3 \mathrm{mg} \mathrm{kg}^{-1}$ na amostra de cigarro de palha CPVC a 187,5 $\pm 0,3 \mathrm{mg} \mathrm{kg}^{-1}$ na amostra de fumo de corda FCA5. A FIG. 4.48 apresenta a variação da concentração do elemento Sb nos grupos amostrados; uma grande variação nas concentrações pode ser observada nos grupos de amostras de cigarro de palha, charuto e fumo de corda.

FIGURA 4.48 - Variação da concentração do elemento Sr $\left(\mathrm{mg} \mathrm{kg}^{-1}\right)$ nos derivados da Nicotiana tabacum L. pela técnica EDXRF.

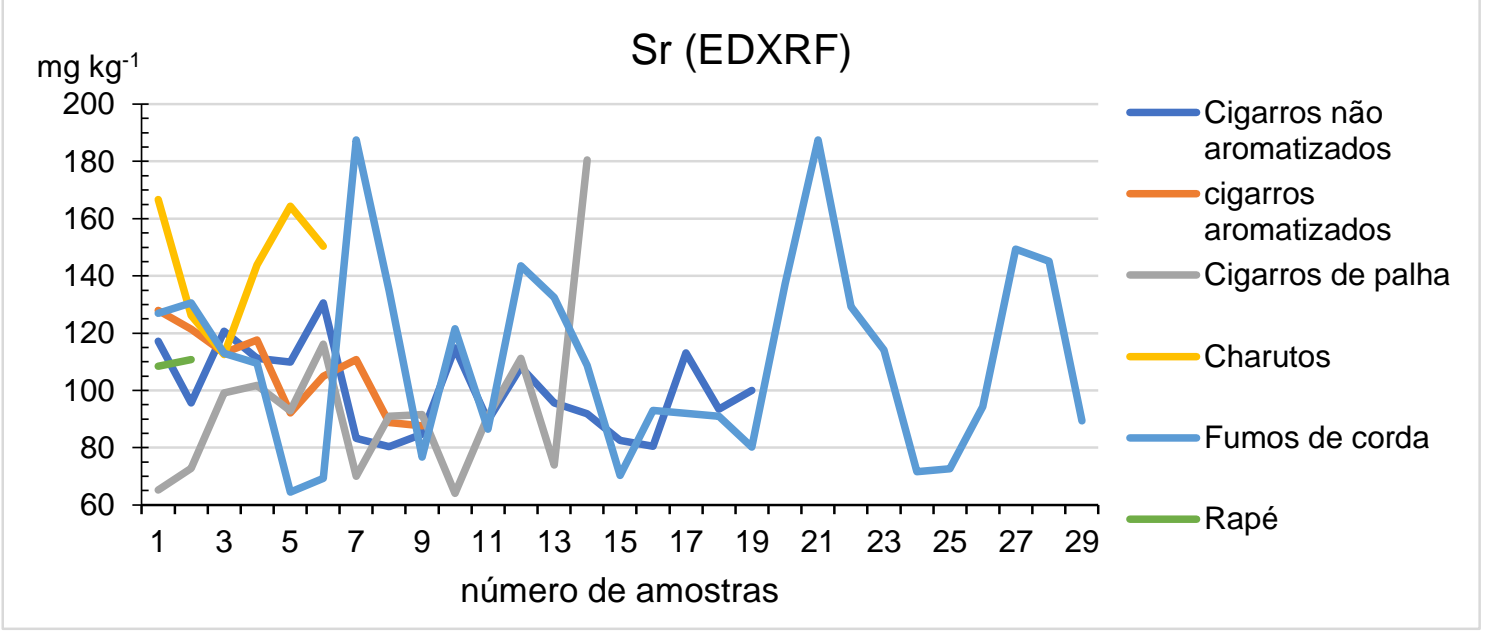


A concentração do elemento Th variou de $0,037 \pm 0,001 \mathrm{mg} \mathrm{kg}^{-1} \mathrm{na}$ amostra de fumo de corda FCA5 a 0,133 $\pm 0,002 \mathrm{mg} \mathrm{kg}^{-1}$ na amostra de fumo de corda, FCDTT. Na FIG 4.49 pode-se observar as variações das concentrações de Th em todo grupo amostral; o grupo de amostras que apresentou a maior variação nas concentrações do elemento Th foi o de fumo de corda.

FIGURA 4.49 - Variação da concentração do elemento Th $\left(\mathrm{mg} \mathrm{kg}^{-1}\right)$ nos derivados da Nicotiana tabacum L. pela técnica EDXRF.

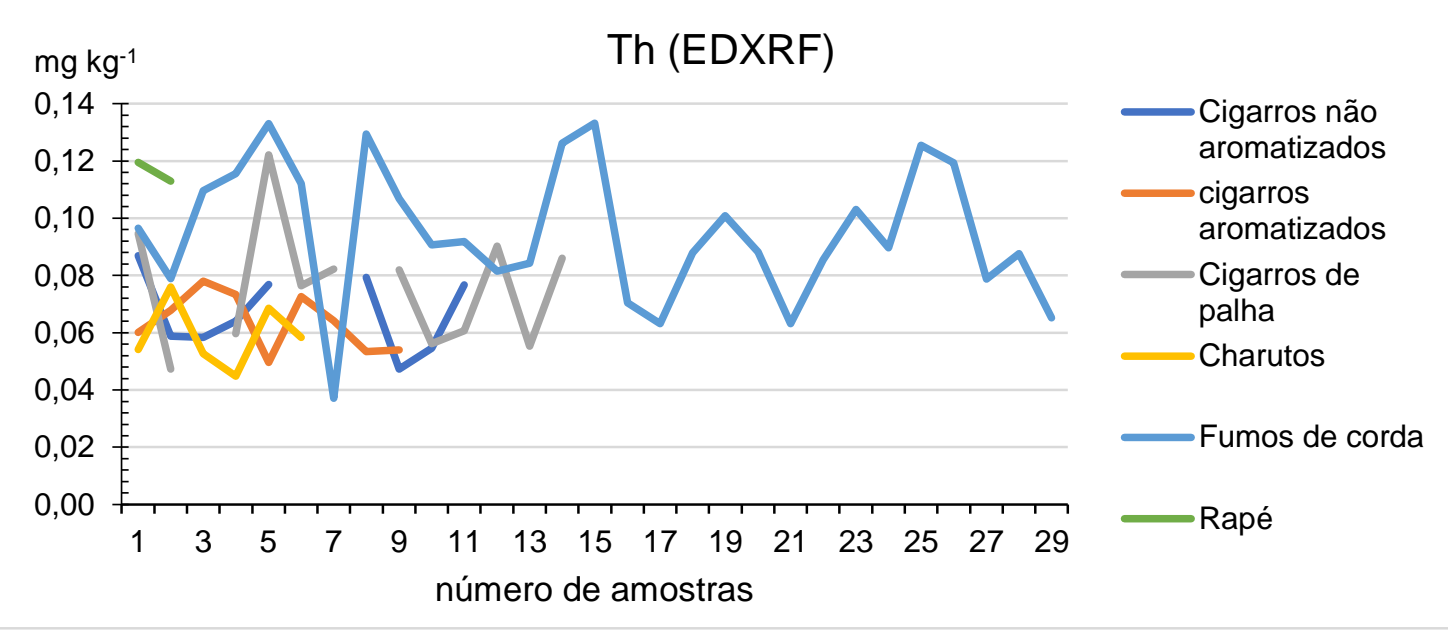

A concentração do elemento $U$ variou de $0,10 \pm 0,02 \mathrm{mg} \mathrm{kg}^{-1}$ na amostra de cigarro não aromatizado CCMS a 0,37 $\pm 0,01 \mathrm{mg} \mathrm{kg}^{-1}$ na amostra de fumo de corda, FCC. Na FIG 4.50 pode-se observar as variações das concentrações de $U$ em todo grupo amostral.

FIGURA 4.50 - Variação da concentração do elemento $U\left(\mathrm{mg} \mathrm{kg}^{-1}\right)$ nos derivados da Nicotiana tabacum L. pela técnica EDXRF.

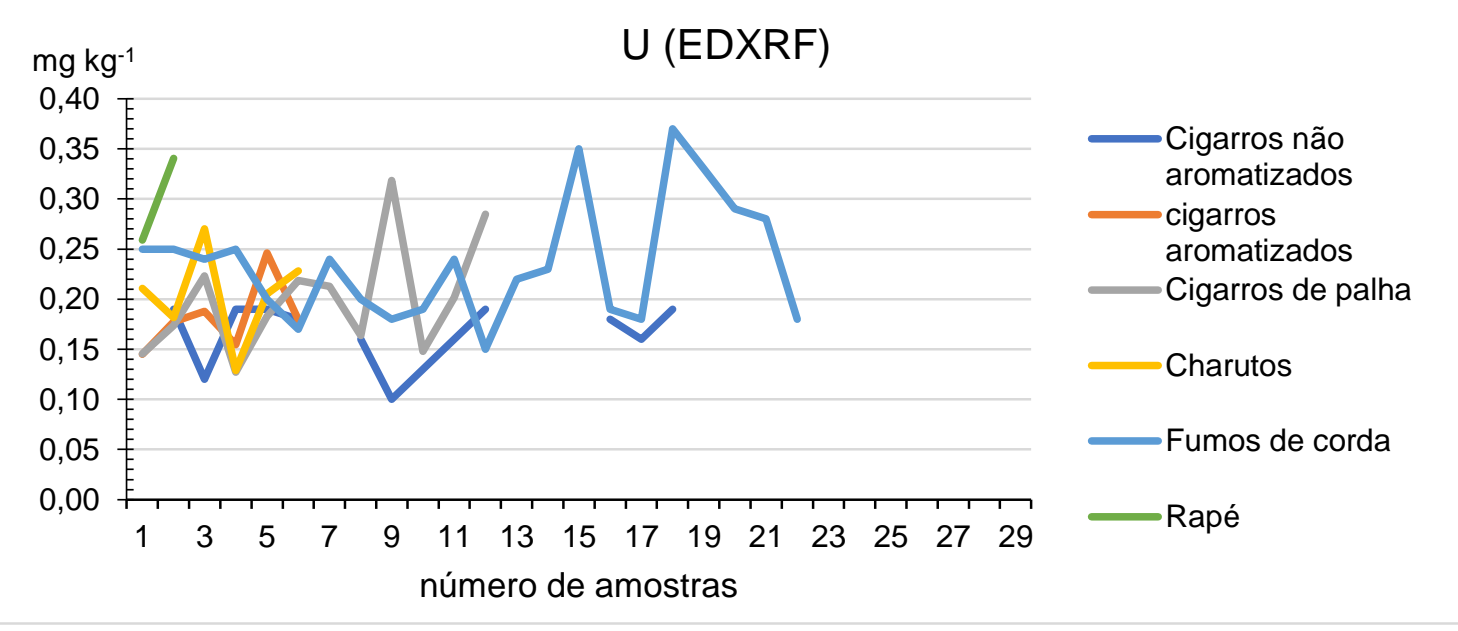


A concentração do elemento $V$ variou de $0,40 \pm 0,05 \mathrm{mg} \mathrm{kg}^{-1}$ na amostra de fumo de corda FCCPR a 3,72 $\pm 0,05 \mathrm{mg} \mathrm{kg}^{-1}$ na amostra de cigarro de palha CPPG. A FIG. 4.51 apresenta a variação da concentração do elemento $\vee$ em todo grupo amostral; os grupos de amostras que apresentaram maiores variações foram os de cigarro não aromatizado, cigarro de palha, charuto e fumo de corda.

FIGURA 4.51 - Variação da concentração do elemento $\vee\left(\mathrm{mg} \mathrm{kg}^{-1}\right)$ nos derivados da Nicotiana tabacum L. pela técnica EDXRF.

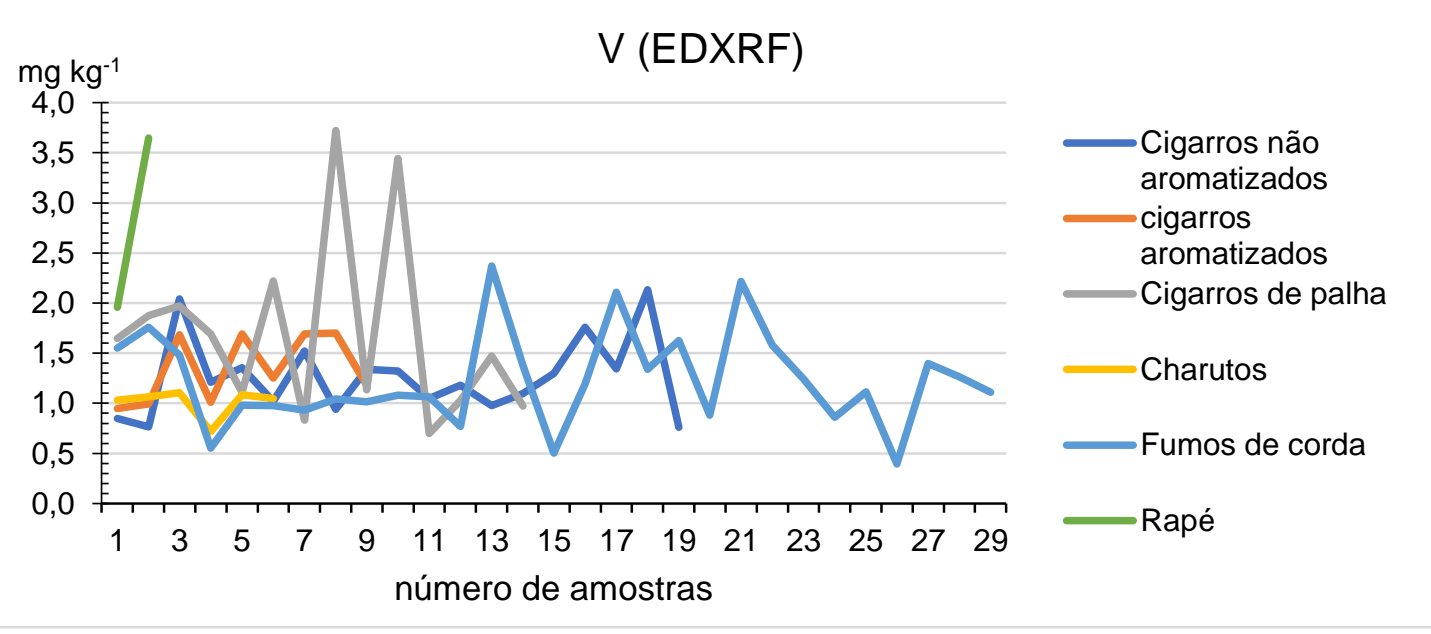

A concentração do elemento $\mathrm{Zn}$ variou de 9,4 \pm 0,2 $\mathrm{mg} \mathrm{kg}^{-1}$ na amostra de cigarro aromatizado CALSF a 27,7 $\pm 0,3 \mathrm{mg} \mathrm{kg}^{-1}$ na amostra de fumo de corda, FCA10. Na FIG 4.52 pode-se observar as variações das concentrações de Zn no grupo amostral.

FIGURA 4.52 - Variação da concentração do elemento $U\left(\mathrm{mg} \mathrm{kg}^{-1}\right)$ nos derivados da Nicotiana tabacum L. pela técnica EDXRF

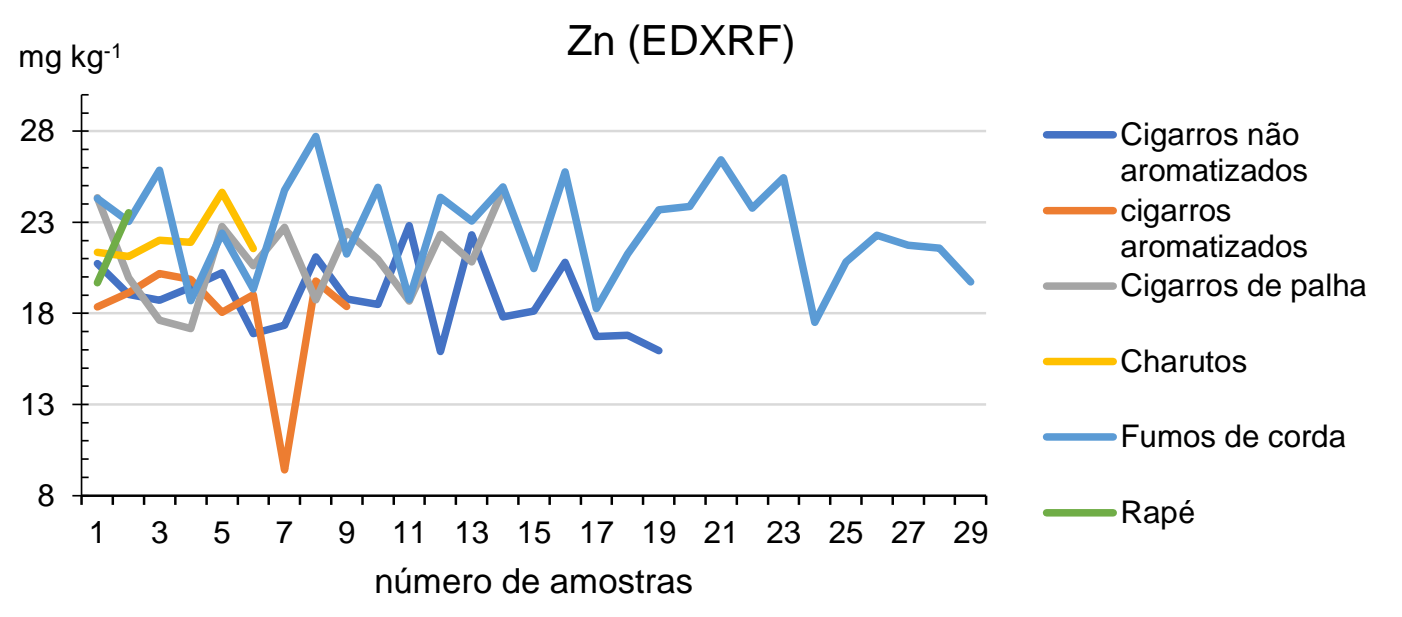




\subsubsection{Elementos Terras Raras La, Nd e Sm}

Na TAB. 4.23 são apresentados os valores mínimo, máximo e média de concentração $\left(\mathrm{mg} \mathrm{kg}^{-1}\right)$ dos elementos La, $\mathrm{Nd}$ e Sm, nas amostras de cigarros não aromatizados, cigarros aromatizados, cigarros de palha, charutos, fumos de corda e rapés.

Entre os elementos terras raras determinados, o elemento La, apresentou os maiores valores de concentração que variaram de 1,8 \pm 0,2 $\mathrm{mg} \mathrm{kg}^{-1}$ na amostra de cigarro não aromatizado CASH a $39 \pm 2 \mathrm{mg} \mathrm{kg}^{-1}$ na amostra de cigarro de palha CPVC; na FIG 4.53 podem-se observar as variações das concentrações de La no grupo amostral.

O elemento $\mathrm{Nd}$ foi determinado em apenas uma amostra de charuto e o elemento Sm não foi determinado nas amostras de rapé. 
Tab. 4.23 - Valores médios, mínimos e máximos de concentração dos elementos terras raras $\mathrm{La}, \mathrm{Nd}$ e $\mathrm{Sm}\left(\mathrm{mg} \mathrm{kg}^{-1}\right)$ nos derivados da Nicotiana tabacum L., pela técnica de EDXRF

\begin{tabular}{|c|c|c|c|c|}
\hline DERIVADOS & & LA & ND & SM \\
\hline \multirow{3}{*}{$\begin{array}{l}\text { CIGARROS NÃO } \\
\text { AROMATIZADOS }\end{array}$} & Min. & $1,8 \pm 0,2$ & $5,5 \pm 0,4$ & $1,08 \pm 0,03$ \\
\hline & Max. & $16 \pm 1$ & $18 \pm 1$ & $2,3 \pm 0,1$ \\
\hline & Med. & $6,3 \pm 3,8$ & $9,0 \pm 4,0$ & $1,46 \pm 0,47$ \\
\hline \multirow{3}{*}{ CIGARROS AROMATIZADOS } & Min. & $3,0 \pm 0,4$ & $5,2 \pm 0,1$ & $0,93 \pm 0,05$ \\
\hline & Max. & $9,1 \pm 0,5$ & $6,5 \pm 0,2$ & $1,8 \pm 0,1$ \\
\hline & Med. & $6,3 \pm 2,4$ & $5,4 \pm 0,9$ & $1,27 \pm 0,39$ \\
\hline \multirow{3}{*}{ CIGARROS DE PALHA } & Min. & $9,1 \pm 0,2$ & $5,4 \pm 0,1$ & $1,3 \pm 0,2$ \\
\hline & Max. & $39 \pm 2$ & $8,4 \pm 0,4$ & $3,3 \pm 0,4$ \\
\hline & Med. & $19,1 \pm 10,7$ & $7,0 \pm 2,3$ & $2,2 \pm 0,9$ \\
\hline \multirow{3}{*}{ CHARUTOS } & Min. & $6,3 \pm 0,2$ & $<5,02$ & $1,52 \pm 0,02$ \\
\hline & Max. & $12,8 \pm 0,4$ & $5,8 \pm 0,5$ & $2,11 \pm 0,05$ \\
\hline & Med. & $8,4 \pm 2,3$ & - & $1,77 \pm 0,31$ \\
\hline \multirow{3}{*}{ FUMOS DE CORDA } & Min. & $2,1 \pm 0,3$ & $5,8 \pm 0,4$ & $1,0 \pm 0,2$ \\
\hline & Max. & $26,8 \pm 0,5$ & $13,3 \pm 0,4$ & $5,4 \pm 0,5$ \\
\hline & Med. & $10,6 \pm 5,4$ & $8,3 \pm 2,6$ & $2,5 \pm 1,4$ \\
\hline \multirow{3}{*}{ RAPÉS } & Min. & $4,5 \pm 0,4$ & $7,66 \pm 0,05$ & $<0,87$ \\
\hline & Max. & $7,1 \pm 0,4$ & $9,31 \pm 0,05$ & $<0,87$ \\
\hline & Med. & $5,8 \pm 1,8$ & $8,49 \pm 1,17$ & - \\
\hline
\end{tabular}

- não determinado

FIGURA 4.53 - Variação da concentração do elemento La $\left(\mathrm{mg} \mathrm{kg}^{-1}\right)$ nos derivados da Nicotiana tabacum L. pela técnica EDXRF




A concentração do elemento $\mathrm{Nd}$ variou de 5,2 \pm 0,1 $\mathrm{mg} \mathrm{kg}^{-1}$ na amostra de cigarro aromatizado CALSA a $18 \pm 1 \mathrm{mg} \mathrm{kg}^{-1}$ na amostra de cigarro não aromatizado CCDA. Na FIG 4.54 podem-se observar as variações das concentrações de La no grupo amostral.

FIGURA 4.54 - Variação da concentração do elemento $\mathrm{Nd}\left(\mathrm{mg} \mathrm{kg}^{-1}\right)$ nos derivados da Nicotiana tabacum $\mathrm{L}$. pela técnica EDXRF

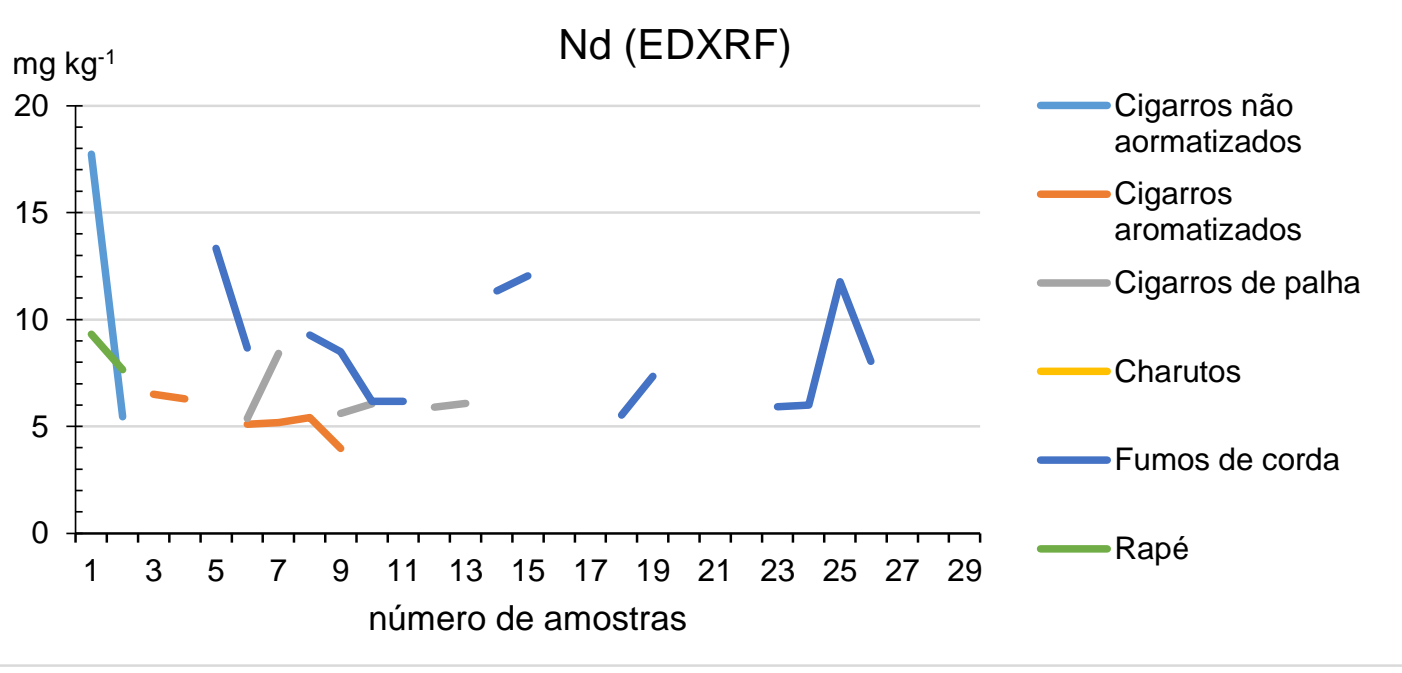

A concentração do elemento $\mathrm{Sm}$ variou de 0,93 $\pm 0,06 \mathrm{mg} \mathrm{kg}^{-1}$ na amostra de cigarro aromatizado CALAM a 5,4 \pm 0,5 $\mathrm{mg} \mathrm{kg}^{-1}$ na amostra de fumo de corda FCDR. Na FIG 4.55 podem-se observar as variações das concentrações de Sm no grupo amostral.

FIGURA 4.55 - Variação da concentração do elemento Sm $\left(\mathrm{mg} \mathrm{kg}^{-1}\right)$ nos derivados da Nicotiana tabacum L. pela técnica EDXR




Martinez et al. 2008 analisaram 9 marcas de cigarros mexicanos pela técnica de Fluorescência de raio-X por Reflexão Total e concluíram que os dados obtidos pelo método são precisos e reprodutíveis e fornecem a vantagem de análise de alguns elementos que não podem ser detectados pela técnica de INAA. A TAB 4.24 compara os resultados obtidos pela técnica de EDXRF com os resultados obtidos pelos autores.

TABELA 4.24 - Valores médios de concentração determinados no presente trabalho e em cigarros mexicanos $\left(\mathrm{mg} \mathrm{kg}^{-1}\right)$

\begin{tabular}{c|c|cc}
\hline Elementos & $\begin{array}{c}\text { MARTINEZ et } \\
\text { al.(2008) }\end{array}$ & \multicolumn{2}{|c}{ Presente estudo } \\
\hline $\mathrm{Cigarros} \mathrm{mexicanos}$ & $\begin{array}{c}\text { Cigarros não } \\
\text { aromatizados }\end{array}$ & $\begin{array}{c}\text { Cigarros } \\
\text { aromatizados }\end{array}$ \\
\hline${ }^{*} \mathrm{Ca}$ & 11 & 55 & 50 \\
\hline $\mathrm{Cu}$ & 3 & 1,7 & 1,7 \\
\hline $\mathrm{Fe}$ & 14 & 4,9 & 5,0 \\
\hline $\mathrm{Mn}$ & 485 & 583 & 595 \\
\hline $\mathrm{Ni}$ & 108 & 188 & 191 \\
\hline $\mathrm{Pb}$ & 2 & 1,3 & 1,1 \\
\hline $\mathrm{Rb}$ & 1 & 1,0 & 20 \\
\hline $\mathrm{Sr}$ & 20 & 20 & 107 \\
\hline $\mathrm{Zn}$ & 130 & 100 & 18 \\
\hline
\end{tabular}

${ }^{\star} \mathrm{Ca}$ em \%

Os elementos $\mathrm{Br}$ e $\mathrm{Mn}$ apresentaram concentrações maiores das determinadas em cigarros mexicanos e os elementos $\mathrm{Ca}, \mathrm{Cu}, \mathrm{Ni}$, e $\mathrm{Zn}$ apresentaram valores menores do que os encontrados pelos autores.

\subsubsection{Radionuclídeos Naturais ${ }^{226} \mathrm{Ra},{ }^{210} \mathrm{~Pb},{ }^{210} \mathrm{Po},{ }^{238} \mathrm{U},{ }^{228} \mathrm{Ra}$ e ${ }^{232} \mathrm{Th}$}

Na TAB. 4.25 são apresentados os valores de concentrações mínimo, máximo e média dos radionuclídeos ${ }^{226} \mathrm{Ra},{ }^{210} \mathrm{~Pb},{ }^{210} \mathrm{Po},{ }^{238} \mathrm{U},{ }^{228} \mathrm{Ra}$ e ${ }^{232} \mathrm{Th}$ em mBq $\mathrm{g}^{-1}$ nas amostras de cigarros não aromatizados, cigarros aromatizados, cigarros de palha, charutos, fumos de corda e rapé.

Os resultados de concentração dos radionuclídeos naturais ${ }^{238} \mathrm{U}$ e ${ }^{232} \mathrm{Th}$ obtidos por EDXRF foram convertidos de $\mathrm{mg} \mathrm{kg}^{-1}$ para $\mathrm{mBq} \mathrm{g} \mathrm{g}^{-1}$ usando a expressão (5.1) (DAMATTO, 2010). 


$$
A=\frac{0,693 \cdot m \cdot N a}{T_{1 / 2} \cdot M A}
$$

Em que:
A: atividade especifica $\left(\mathrm{Bq} \mathrm{g}^{-1}\right)$;
$\mathrm{m}$ : massa de amostra (g)

Na: número de Avogrado;

MA: massa atômica (g);

$\mathrm{T}_{1 / 2}$ : meia vida do radionuclídeo

TABELA 4.25 - Valores médios mínimos e máximos de concentração do radionuclídeos naturais $\left(\mathrm{mBq} \mathrm{g}^{-1}\right)$

\begin{tabular}{|c|c|c|c|c|c|c|c|}
\hline \multicolumn{2}{|c|}{ DERIVADOS } & ${ }^{226} \mathrm{Ra}$ & ${ }^{210} \mathrm{~Pb}$ & ${ }^{210} \mathrm{Po}$ & ${ }^{238} U$ & ${ }^{228} \mathrm{Ra}$ & ${ }^{232} \mathrm{Th}$ \\
\hline \multirow{3}{*}{$\begin{array}{c}\text { Cigarros não } \\
\text { aromatizados }\end{array}$} & Min. & $7 \pm 1$ & $15 \pm 5$ & $14 \pm 1$ & $1,3 \pm 0,1$ & $34 \pm 2$ & $0,066 \pm 0,004$ \\
\hline & Max. & $13 \pm 2$ & $26 \pm 2$ & $25 \pm 1$ & $2,4 \pm 0,1$ & $45 \pm 6$ & $0,40 \pm 0,01$ \\
\hline & Med. & $10 \pm 2$ & $21 \pm 3$ & $21 \pm 1$ & $2,0 \pm 0,4$ & $40 \pm 5$ & $0,228 \pm 0,105$ \\
\hline \multirow{3}{*}{$\begin{array}{c}\text { Cigarros } \\
\text { aromatizados }\end{array}$} & Min. & $4,7 \pm 0,2$ & $<4,9$ & $6,3 \pm 0,5$ & $1,8 \pm 0,4$ & $26 \pm 5$ & $0,202 \pm 0,004$ \\
\hline & Max. & $14 \pm 1$ & $25 \pm 2$ & $22 \pm 1$ & $3,1 \pm 0,4$ & $69 \pm 6$ & $0,318 \pm 0,004$ \\
\hline & Med. & $9 \pm 4$ & $18 \pm 4$ & $16 \pm 1$ & $2,4 \pm 0,4$ & $40 \pm 12$ & $0,259 \pm 0,039$ \\
\hline \multirow{3}{*}{$\begin{array}{c}\text { Cigarros de } \\
\text { palha }\end{array}$} & Min. & $6 \pm 2$ & $17 \pm 2$ & $11 \pm 1$ & $1,6 \pm 0,1$ & $37 \pm 3$ & $0,128 \pm 0,004$ \\
\hline & Max. & $22 \pm 7$ & $55 \pm 5$ & $35 \pm 2$ & $4,1 \pm 0,1$ & $81 \pm 4$ & $0,498 \pm 0,004$ \\
\hline & Med. & $12 \pm 2$ & $30 \pm 4$ & $19 \pm 1$ & $2,6 \pm 0,8$ & $57 \pm 4$ & $0,285 \pm 0,103$ \\
\hline \multirow{3}{*}{ Charutos } & Min. & $18 \pm 1$ & $6,2 \pm 0,5$ & $15 \pm 1$ & $1,6 \pm 0,3$ & $52 \pm 3$ & $0,183 \pm 0,004$ \\
\hline & Max. & $28 \pm 1$ & $22 \pm 2$ & $22 \pm 1$ & $3,4 \pm 0,6$ & $78 \pm 4$ & $0,310 \pm 0,004$ \\
\hline & Med. & $23 \pm 1$ & $13 \pm 1$ & $18 \pm 1$ & $2,6 \pm 06$ & $63 \pm 3$ & $0,241 \pm 0,046$ \\
\hline \multirow{3}{*}{$\begin{array}{l}\text { Fumos de } \\
\text { corda }\end{array}$} & Min. & $6 \pm 2$ & $5 \pm 1$ & $10 \pm 1$ & $1,9 \pm 0,4$ & $28 \pm 6$ & $0,151 \pm 0,004$ \\
\hline & Max. & $40 \pm 6$ & $78 \pm 2$ & $28 \pm 1$ & $4,6 \pm 0,1$ & $152 \pm 1$ & $0,543 \pm 0,01$ \\
\hline & Med. & $12 \pm 2$ & $35 \pm 3$ & $18 \pm 1$ & $2,9 \pm 0,7$ & $48 \pm 5$ & $0,387 \pm 0,097$ \\
\hline \multirow{3}{*}{ Rapés } & Min. & $19 \pm 1$ & $24 \pm 2$ & $22 \pm 2$ & $3,2 \pm 0,1$ & $35 \pm 2$ & $0,487 \pm 0,004$ \\
\hline & Max. & $17 \pm 1$ & $30 \pm 3$ & $29 \pm 3$ & $4,3 \pm 0,1$ & $34 \pm 2$ & $0,460 \pm 0,004$ \\
\hline & Med. & $18 \pm 1$ & $27 \pm 4$ & $28 \pm 1$ & $3,7 \pm 0,7$ & $35 \pm 1$ & $0,474 \pm 0,019$ \\
\hline
\end{tabular}


A concentração do radionuclídeo ${ }^{226} \mathrm{Ra}$ variou de $4,7 \pm 0,2 \mathrm{mBq} \mathrm{g}^{-1}$ na amostra de cigarro não aromatizado CALAM a $40 \pm 6 \mathrm{mBq} \mathrm{g}^{-1}$ na amostra de fumo de corda FCAAL; as maiores concentrações de ${ }^{226} \mathrm{Ra}$ foram determinadas em duas amostras de cigarro de palha e em uma amostra de fumo de corda. ABD EL - AZIZ, (2005) determinou em amostras de tabaco uma concentração do radionuclídeo ${ }^{226} \mathrm{R}$ de $6,98 \pm 0,56 \mathrm{mBq} \mathrm{g}^{-1}$ e SILVA (2015) encontrou valores de concentração entre $10,4 \pm 0,7 \mathrm{mBq} \mathrm{g}^{-1}$ a 16,1 $\pm 0,9 \mathrm{mBq} \mathrm{g}^{-1}$, valores da mesma ordem de grandeza do presente trabalho.

Na FIG. 4.56 pode-se observar as variações da concentração do radionuclídeo ${ }^{226} \mathrm{Ra}$ nas amostras analisadas.

FIGURA 4.56 - Variação da concentração do radionuclídeo ${ }^{226} \mathrm{Ra}\left(\mathrm{mBq} \mathrm{g}{ }^{-1}\right)$ nos derivados da Nicotiana tabacum L.

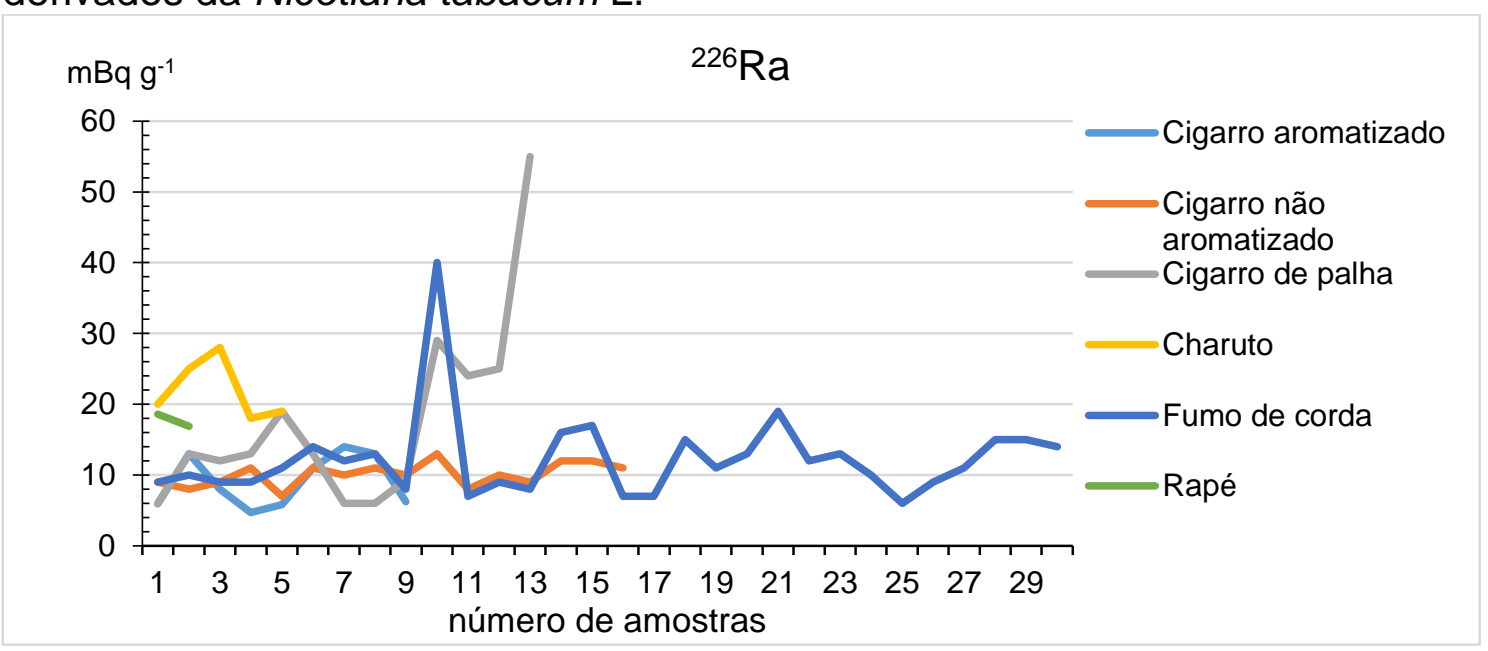

A concentração do radionuclídeo ${ }^{210} \mathrm{~Pb}$ variou de $4,9 \pm 0,4 \mathrm{mg} \mathrm{kg}^{-1}$ (LID da metodologia) na amostra de cigarro aromatizado CALAM a $78 \pm 2 \mathrm{mg} \mathrm{kg}^{-1} \mathrm{na}$ amostra de fumo de corda FCA9.

Os valores máximos de concentração determinados para o radionuclídeo ${ }^{210} \mathrm{~Pb}$ no presente trabalho são muito superiores aos determinados por ABD EL-AZIZ et al (2005) que analisaram amostras de tabaco por espectrometria gama e obtiveram valores médios de 15,62 \pm 1,2 Bq / kg e SILVA (2015) que determinou nas folhas de tabaco uma concentração média de $14 \pm 1$ $\mathrm{mBq} \mathrm{g}^{-1}$. 
Na FIG 4.57 podem-se observar as variações das concentrações do radionuclídeo ${ }^{210} \mathrm{~Pb}$ no grupo amostral.

FIGURA 4.57 - Variação da concentração do radionuclídeo ${ }^{210} \mathrm{~Pb}\left(\mathrm{mBq} \mathrm{g}{ }^{-1}\right)$ nos derivados da Nicotiana tabacum L.

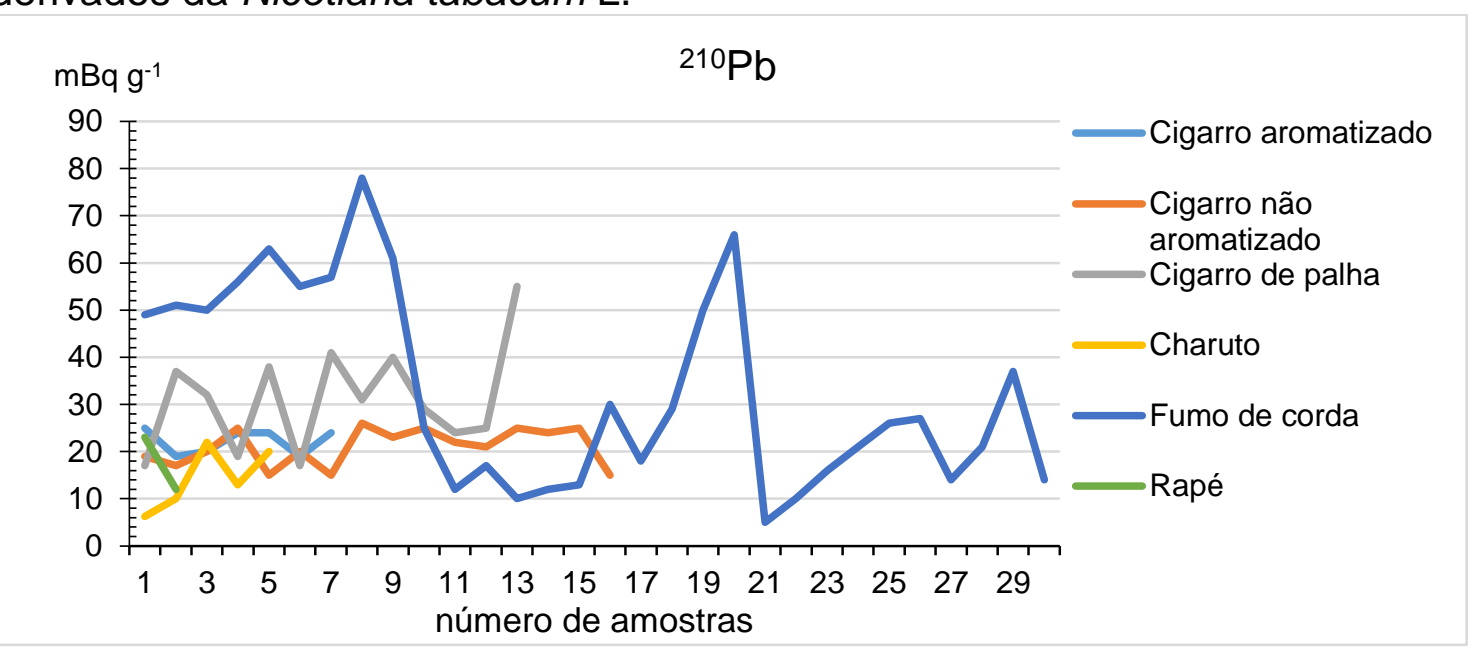

A concentração do radionuclídeo ${ }^{210} \mathrm{Po}$ variou de 6,3 $\pm 0,5 \mathrm{mB} \mathrm{g}^{-1}$ na amostra de cigarro aromatizado CADBM a $35 \pm 2 \mathrm{mBq} \mathrm{g}^{-1}$ na amostra de cigarro de palha CPCPC. A FIG. 4.58 apresenta as variações das concentrações do radionuclídeo ${ }^{210} \mathrm{Po}$ no grupo amostral. Nieri Neto (1996) cita em seu estudo que as concentrações de ${ }^{210} \mathrm{Po}$ variam amplamente de $0,04 \mathrm{mBq} \mathrm{g}^{-1}$ a $111 \mathrm{mBq} \mathrm{g}^{-1} \mathrm{em}$ plantas (material seco) e que em folhas de tabaco a concentração pode variar de $5,6 \mathrm{mBq} \mathrm{g}^{-1}$ a $102 \mathrm{mBq} \mathrm{g}^{-1}$. Godoy et al. (1992) determinou uma concentração média de ${ }^{210} \mathrm{Po}$ em folhas de tabaco brasileiro de 22,0 \pm 5,6 $\mathrm{mBq} \mathrm{g}^{-1}$ e Silva (2015) determinou o radionuclídeo também em folhas de tabaco brasileiro e obteve concentrações entre 5,2 e $18 \mathrm{mBq} \mathrm{g}^{-1}$.

Em outros estudos de folhas de tabaco fora do território brasileiro, as concentrações encontradas por Savidou et al. (2006) em amostras gregas variaram entre 3,6 a 17 mBq g-1 e Skwarzec et al. (2001) em amostras polonesas variaram entre 10,4 a $35,0 \mathrm{mBq} \mathrm{g}^{-1}$.

As variações encontradas neste trabalho são próximas das encontradas em amostras de folhas de tabaco da literatura. 
FIGURA 4.58 - Variação da concentração do radionuclídeo ${ }^{210} \mathrm{Po}\left(\mathrm{mBq} \mathrm{g}^{-1}\right)$ nos derivados da Nicotiana tabacum L.



A concentração do radionuclídeo ${ }^{238} \mathrm{U}$ variou de $1,3 \pm 0,1 \mathrm{mB} \mathrm{g}^{-1}$ na amostra de cigarro não aromatizado CCMS a 4,6 \pm 0,1 $\mathrm{mBq} \mathrm{g}^{-1}$ na amostra de fumo de corda FCC. A FIG. 4.59 apresenta as variações das concentrações do radionuclídeo ${ }^{238} \mathrm{U}$ no grupo amostral.

FIGURA 4.59 - Variação da concentração do radionuclídeo ${ }^{238} \mathrm{U}\left(\mathrm{mBq} \mathrm{g} \mathrm{g}^{-1}\right)$ nos derivados da Nicotiana tabacum $\mathrm{L}$.

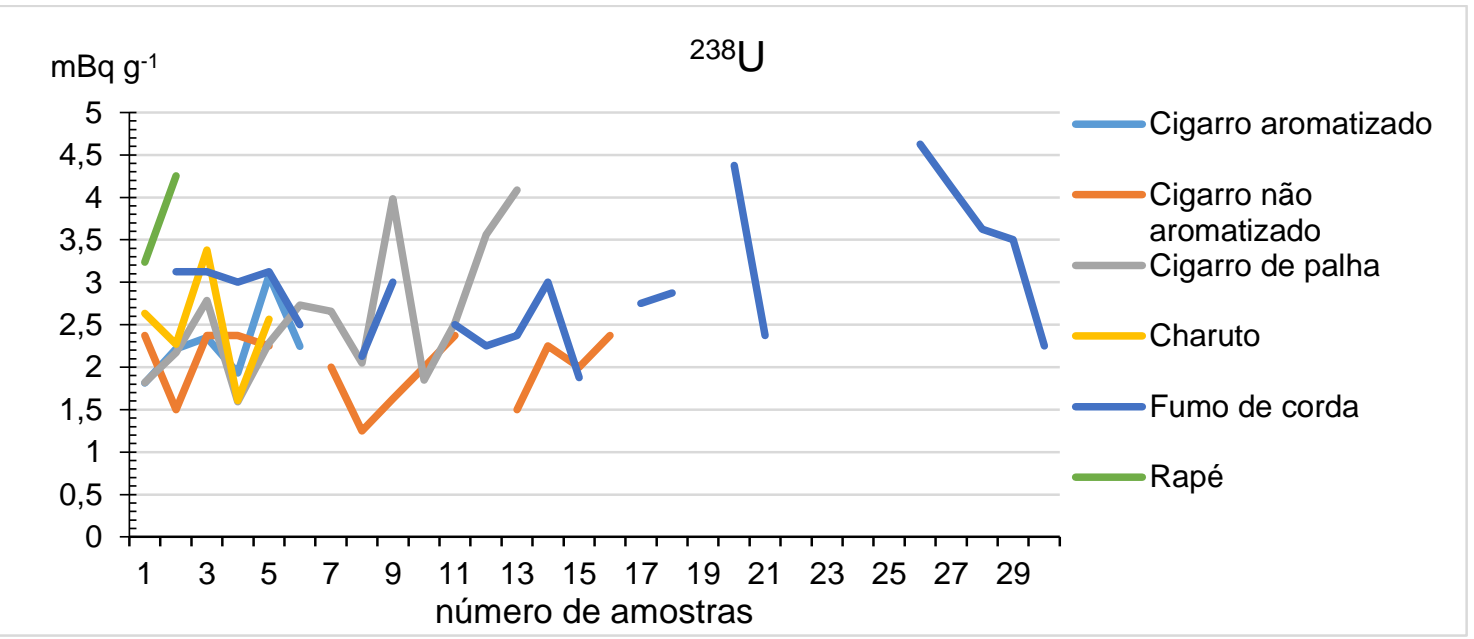

A concentração do radionuclídeo ${ }^{228} \mathrm{Ra}$ variou de $26 \pm 5 \mathrm{mBq} \mathrm{g}^{-1}$ na amostra de cigarro aromatizado CALSF a $152 \pm 1 \mathrm{mBq} \mathrm{g}^{-1}$ na amostra de fumo de corda FCAAL. As concentrações determinadas de ${ }^{228} \mathrm{Ra}$ são da mesma ordem de grandeza dos valores determinados por SILVA (2015) que analisou folhas de tabaco e obteve valores entre $67 \pm 3 \mathrm{mBq} \mathrm{g}{ }^{-1}$ a $103 \pm 4 \mathrm{mBq} \mathrm{g}{ }^{-1}$. A FIG. 4.60 
apresenta as variações das concentrações do radionuclídeo ${ }^{228} \mathrm{Ra}$ no grupo amostral.

FIGURA 4.60 - Variação da concentração do radionuclídeo ${ }^{228} \mathrm{Ra}\left(\mathrm{mBq} \mathrm{g}{ }^{-1}\right)$ nos derivados da Nicotiana tabacum L.

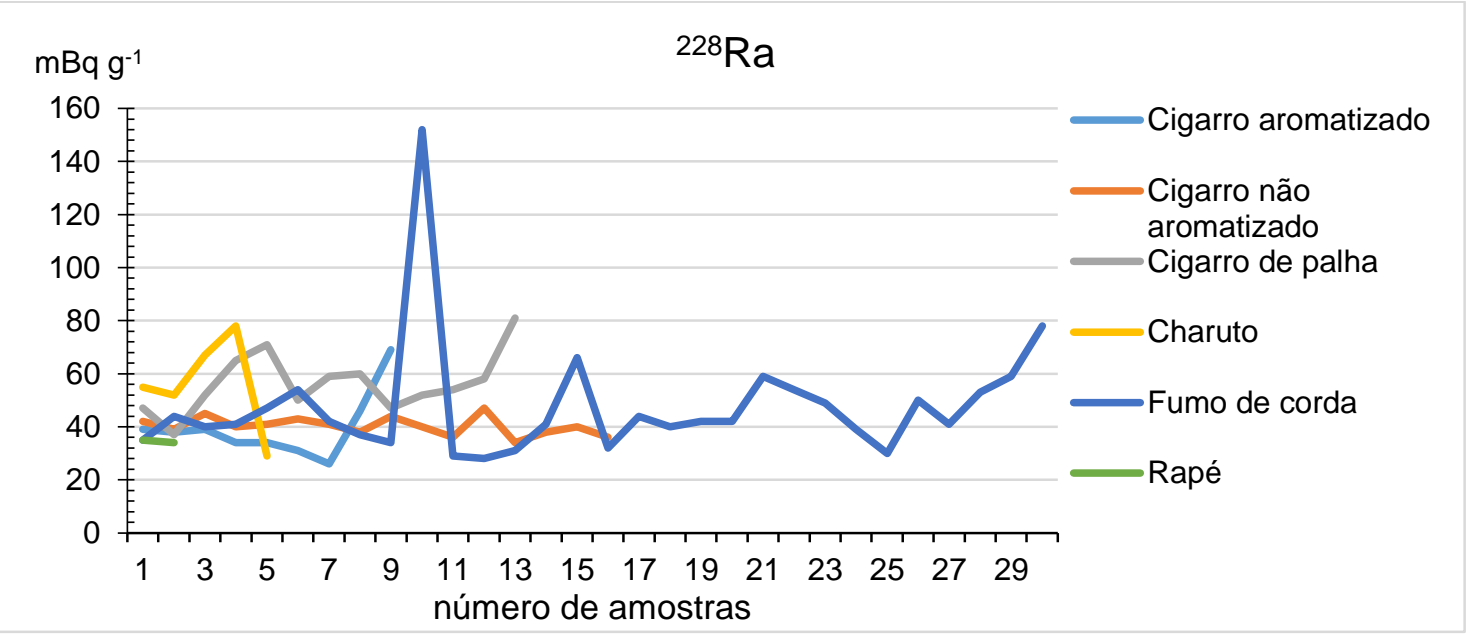

A concentração do radionuclídeo ${ }^{232}$ Th variou de 0,066 $\pm 0,004 \mathrm{mBq} \mathrm{g}^{-1}$ na amostra de cigarro não aromatizado CCDLV a 0,54 $\pm 0,01 \mathrm{mBq} \mathrm{g}^{-1}$ na amostra de fumo de corda FCDTT. SILVA (2015) obteve valores médios de concentração de ${ }^{232}$ Th em folhas de plantas de tabaco, variedade Burley, de 3,0 $\pm 0,6 \mathrm{mBq} \mathrm{g}^{-1}$, os valores muito acima dos determinados no presente trabalho. A FIG. 4.61 apresenta as variações das concentrações do radionuclídeo ${ }^{232}$ Th no grupo amostral.

FIGURA 4.61 - Variação da concentração do radionuclídeo ${ }^{232} \mathrm{Th}\left(\mathrm{mBq} \mathrm{g}{ }^{-1}\right)$ nos derivados da Nicotiana tabacum $\mathrm{L}$.

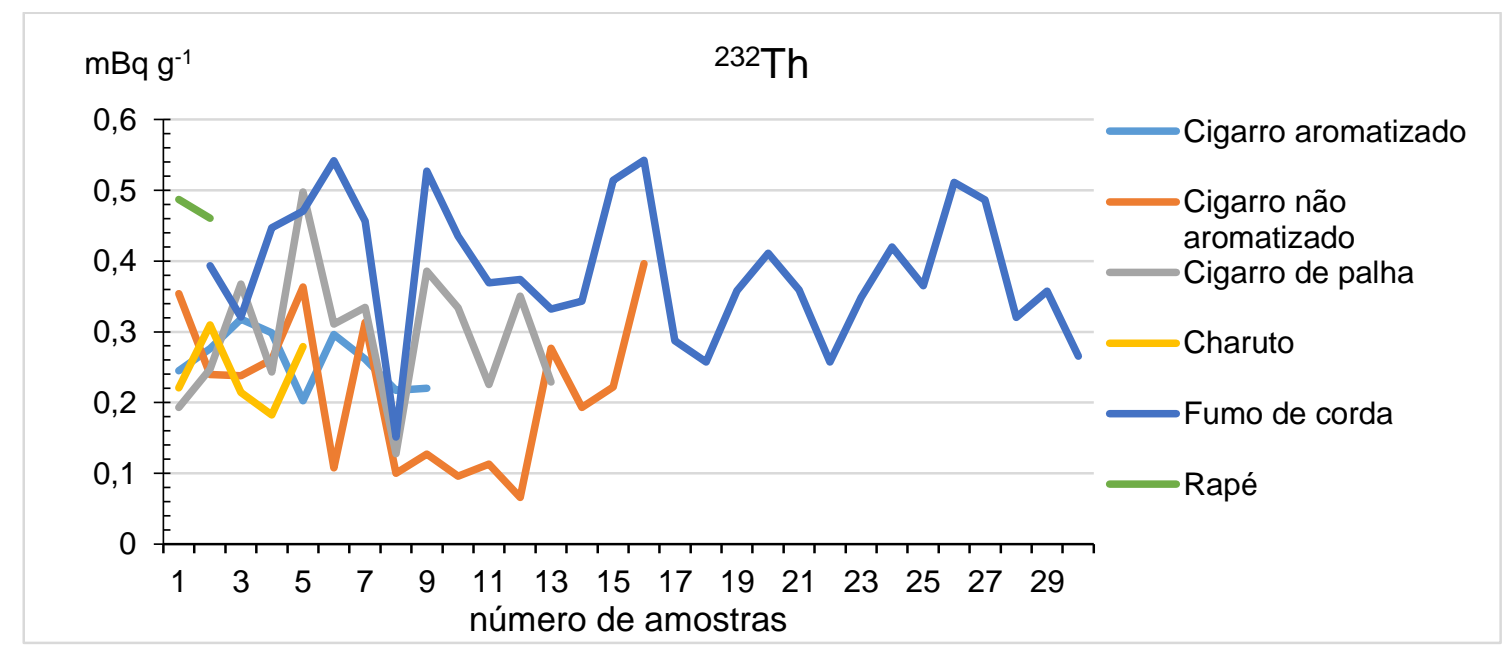


A FIG. 4.62 apresenta as concentrações médias dos radionuclídeos estudados considerando a massa de tabaco de um cigarro de aproximadamente $0,5 \mathrm{~g}$. Embora o radionuclídeo ${ }^{228} \mathrm{Ra}$ da série de decaimento do ${ }^{232} \mathrm{Th}$ tenha apresentado as maiores concentrações, toda a atenção é voltada para os radionuclídeos ${ }^{210} \mathrm{~Pb}$ e ${ }^{210} \mathrm{Po}$, pois eles são volatilizados à temperatura de queima do cigarro e são inalados pelo homem (IWAOKA e YONEHARA, 2012)

Pode-se observar que os radionuclídeos que apresentaram as maiores concentrações em todas as amostras analisadas foram ${ }^{228} \mathrm{Ra},{ }^{210} \mathrm{~Pb}$ e ${ }^{210} \mathrm{Po}$ e as menores concentrações foram obtidas para os radionuclídeos ${ }^{238} \mathrm{U}$ e ${ }^{232} \mathrm{Th}$.

FIGURA 4.62 - Concentração média dos radionuclídeos em mBq cigarro-1.

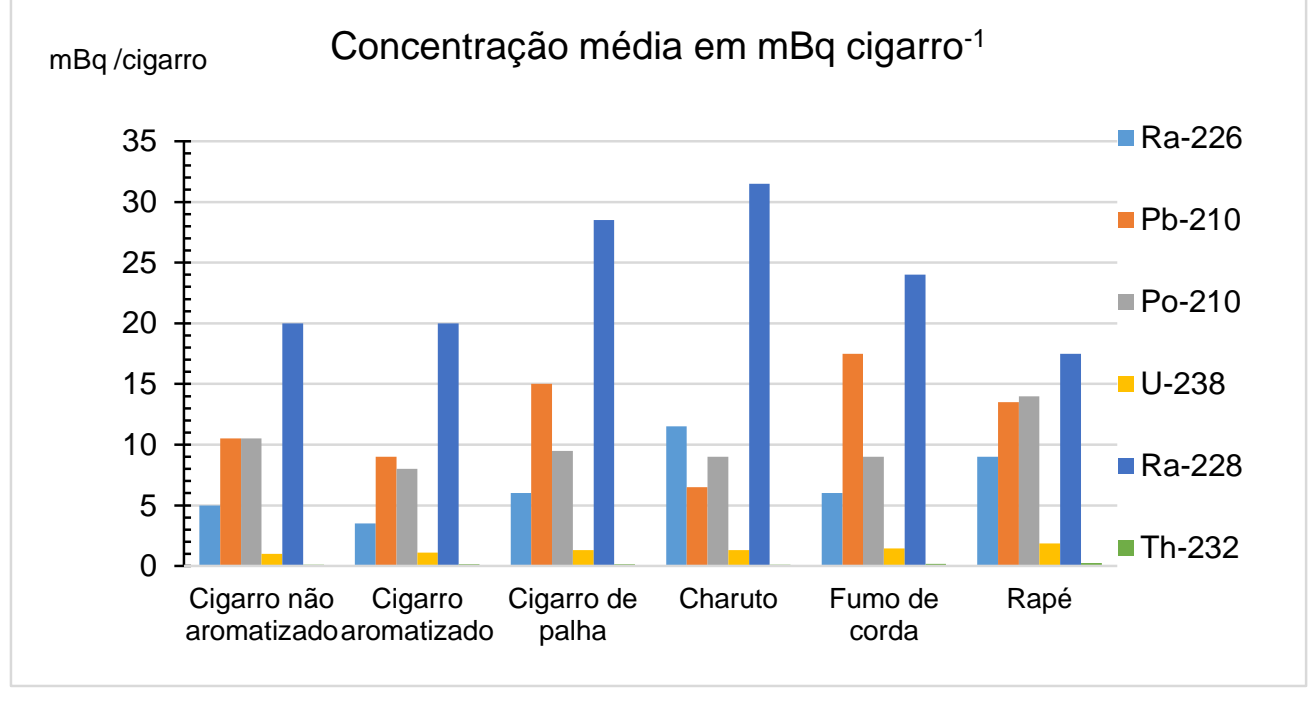

A FIG. 4.63 apresenta os valores de concentração dos radionuclídeos ${ }^{226} \mathrm{Ra},{ }^{210} \mathrm{~Pb}$ e ${ }^{210} \mathrm{Po}$ em $\mathrm{mBq}$ cigarro-1 do presente trabalho e de artigos da literatura. 
FIGURA 4.63 - Concentração dos radionuclídeos ${ }^{226} \mathrm{Ra},{ }^{210} \mathrm{~Pb}$ e ${ }^{210} \mathrm{Po}$ em amostras de cigarros ( $\mathrm{mBq}$ cigarro ${ }^{-1}$ ), presente trabalho e dados da literatura.

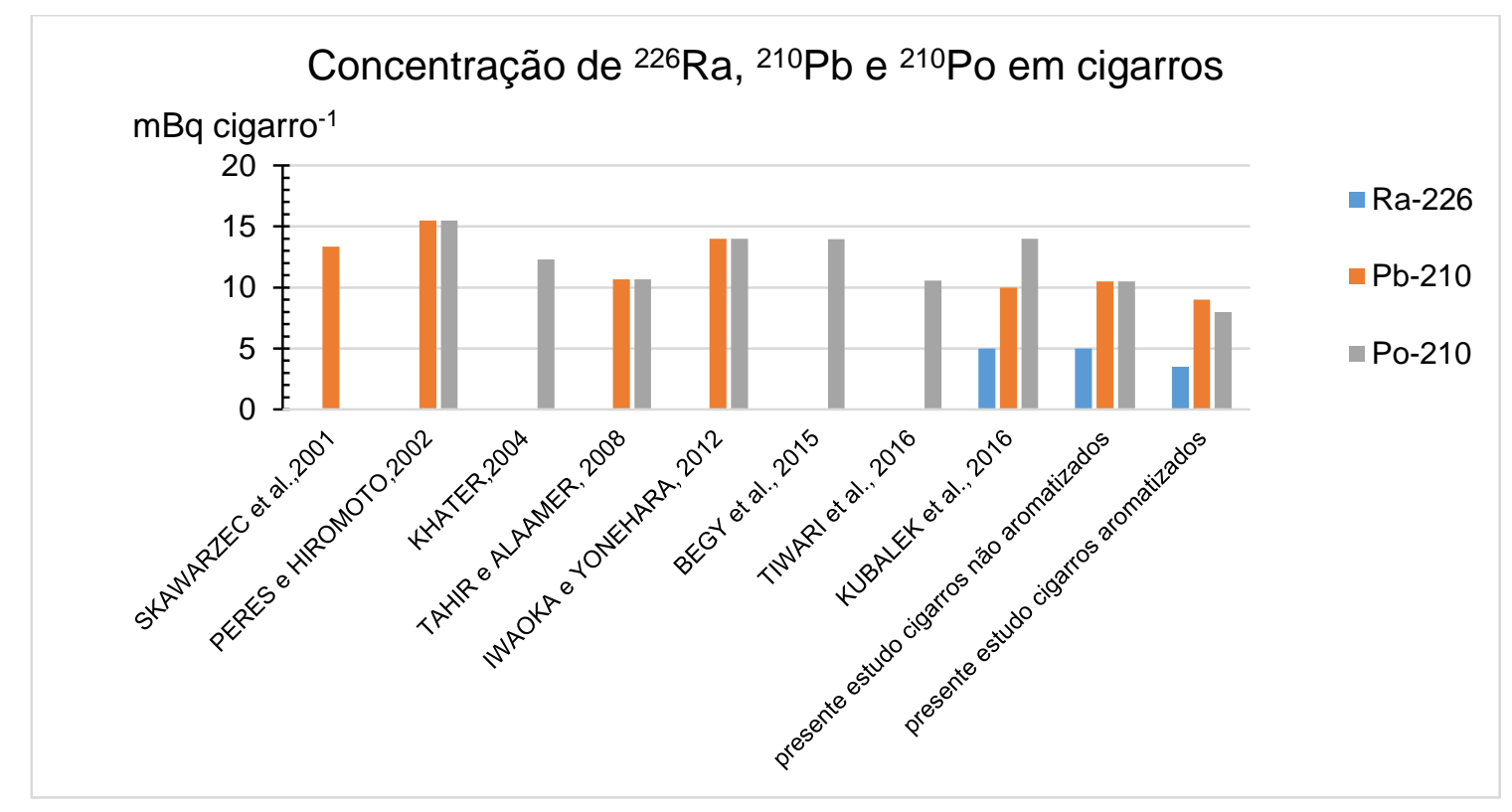

Observa-se na FIG. 4.63 que os valores determinados no presente trabalho são da mesma ordem de grandeza da maioria dos artigos da literatura.

A TAB. 4.25 apresenta os valores de concentração dos radionuclídeos ${ }^{210} \mathrm{~Pb} \mathrm{e}^{210} \mathrm{Po}$ em $\mathrm{mBq} \mathrm{g} \mathrm{g}^{-1}$ do presente trabalho e de Kelecon et al. que determinaram os radionuclídeos em amostras de charutos de diferentes localidades. Observa-se que os valores determinados no presente estudo são da mesma ordem de grandeza da maioria dos locais.

TABELA 4.26 - Concentração dos radionuclídeos ${ }^{210} \mathrm{~Pb}$ e ${ }^{210} \mathrm{Po}\left(\mathrm{mBq} \mathrm{g}{ }^{-1}\right)$ em amostras de charutos.

\begin{tabular}{cccc}
\hline Autor & Origem & ${ }^{\mathbf{2 1 0}} \mathbf{P o}\left(\mathbf{m B q ~} \mathbf{~}^{-1}\right)$ & $\left.{ }^{\mathbf{2 1 0}} \mathbf{P b} \mathbf{~} \mathbf{m B q ~} \mathbf{~ g}^{-1}\right)$ \\
\hline \multirow{3}{*}{$\begin{array}{c}\text { KELECON et al., } \\
2002\end{array}$} & Brasil & $25,7 \pm 4,6$ & $15,2 \pm 6,9$ \\
\cline { 2 - 4 } & Cuba & $13,0 \pm 4,7$ & $6,0 \pm 4,7$ \\
\cline { 2 - 4 } & Republica Dominicana & $24,5 \pm 8,4$ & $11,9 \pm 5,3$ \\
\cline { 2 - 4 } & Europa & $24,6 \pm 8,7$ & $5,1 \pm 2,4$ \\
\cline { 2 - 4 } & América Latina & $34,0 \pm 14,4$ & $12,3 \pm 4,4$ \\
\cline { 2 - 4 } Presente estudo & Estados Unidos & $16,9 \pm 7,3$ & $5,4 \pm 1,6$ \\
\hline
\end{tabular}




\subsubsection{Avaliação da dose}

\subsubsection{Dose estimada}

Para o cálculo da dose estimada, para os radionuclídeos ${ }^{210} \mathrm{~Pb}$ e ${ }^{210} \mathrm{Po}$, foi levado em consideração que um cigarro tem aproximadamente 0,5 $\mathrm{g}$ de tabaco e que um fumante consome um maço de cigarro por dia contendo 20 cigarros, portanto uma massa de $10 \mathrm{~g}$ de tabaco por dia ou 3,65 kg por ano. As frações ingeridas dos radionuclídeos estudados, da fumaça para os pulmões, seguiram os valores da literatura: ${ }^{210} \mathrm{~Pb}$ de 0,5 (TAHIR, 2008) e de ${ }^{210} \mathrm{Po}$ de 0,2 (BEGY, 2015).

Embora as referências sejam somente para os cigarros com filtro e as formas de inalação sejam diferentes para cada tipo de derivado estudado, considerou-se as mesmas frações ingeridas do radionuclídeo para todos os tipos de derivados de tabaco analisados.

Na FIG. 4.64 são apresentados os valores de dose média estimada $\left(\mu \mathrm{Sv}\right.$ ano ${ }^{-1}$ ) para os radionuclídeos ${ }^{210} \mathrm{~Pb}$ e ${ }^{210} \mathrm{Po}$ nos derivados de tabaco analisados.

FIGURA 4.64 - Dose média estimada de ${ }^{210} \mathrm{~Pb}$ e ${ }^{210} \mathrm{Po}(\mu \mathrm{Sv}$ ano-1) nos derivados de tabaco analisados

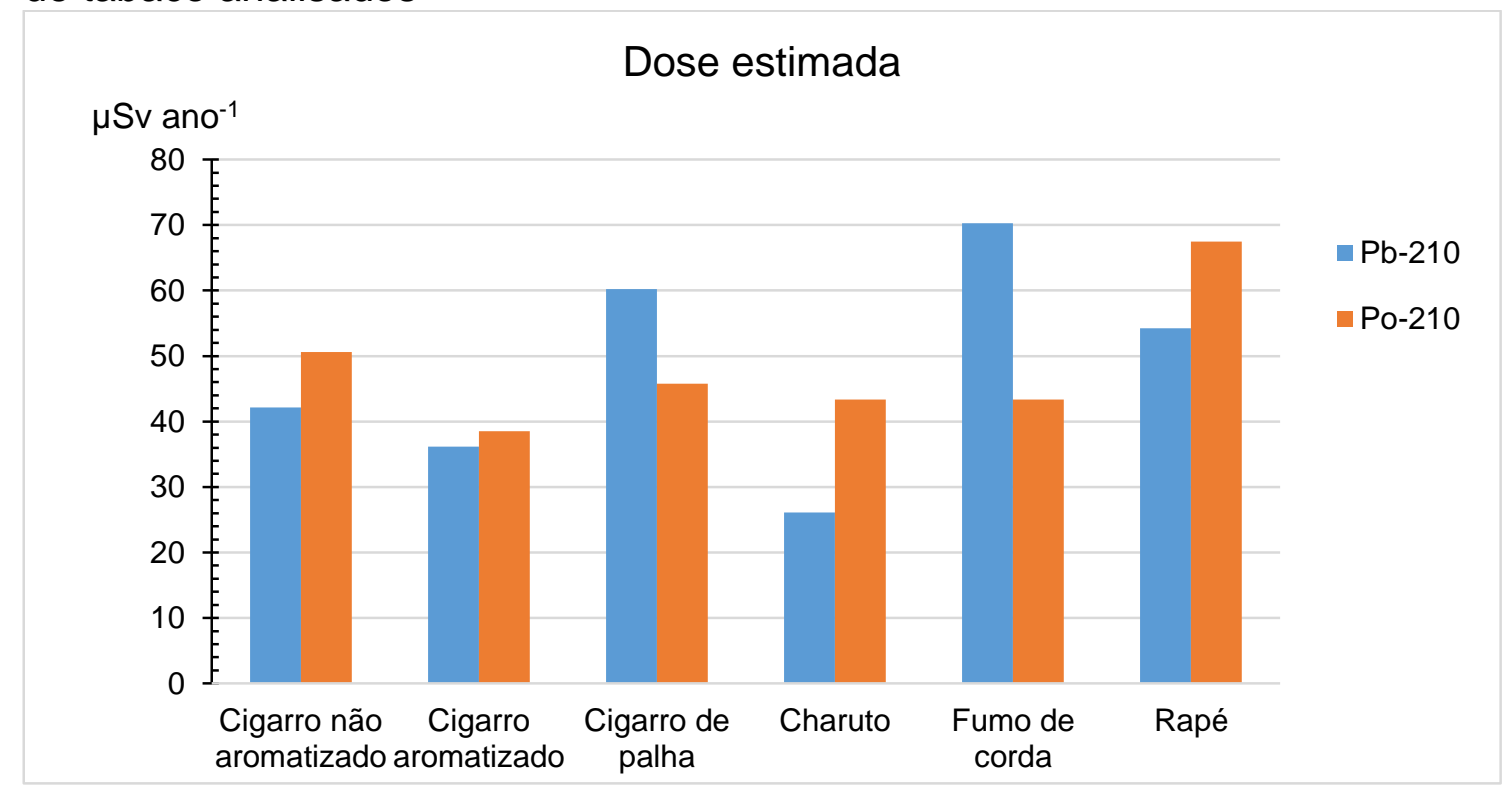


Na FIG. 4.65 e na TAB.4.27 (FIG. 4.65 amostras de cigarro e na TAB.4.25 outros derivados de tabaco e folhas de tabaco) são apresentadas as doses estimadas para os radionuclídeos ${ }^{210} \mathrm{~Pb}$ e ${ }^{210} \mathrm{Po}$ determinadas no presente trabalho e de artigos da literatura. Pode-se observar que os derivados de tabaco estudados no presente trabalho apresentaram valores de dose menores dos determinados na maioria da literatura, mais ainda dentro do intervalo relatado.

FIGURA 4.65 - Dose média estimada de ${ }^{210} \mathrm{~Pb}$ e ${ }^{210} \mathrm{Po}(\mu \mathrm{Sv}$ ano-1) em amostras de cigarro do presente trabalho e da literatura.

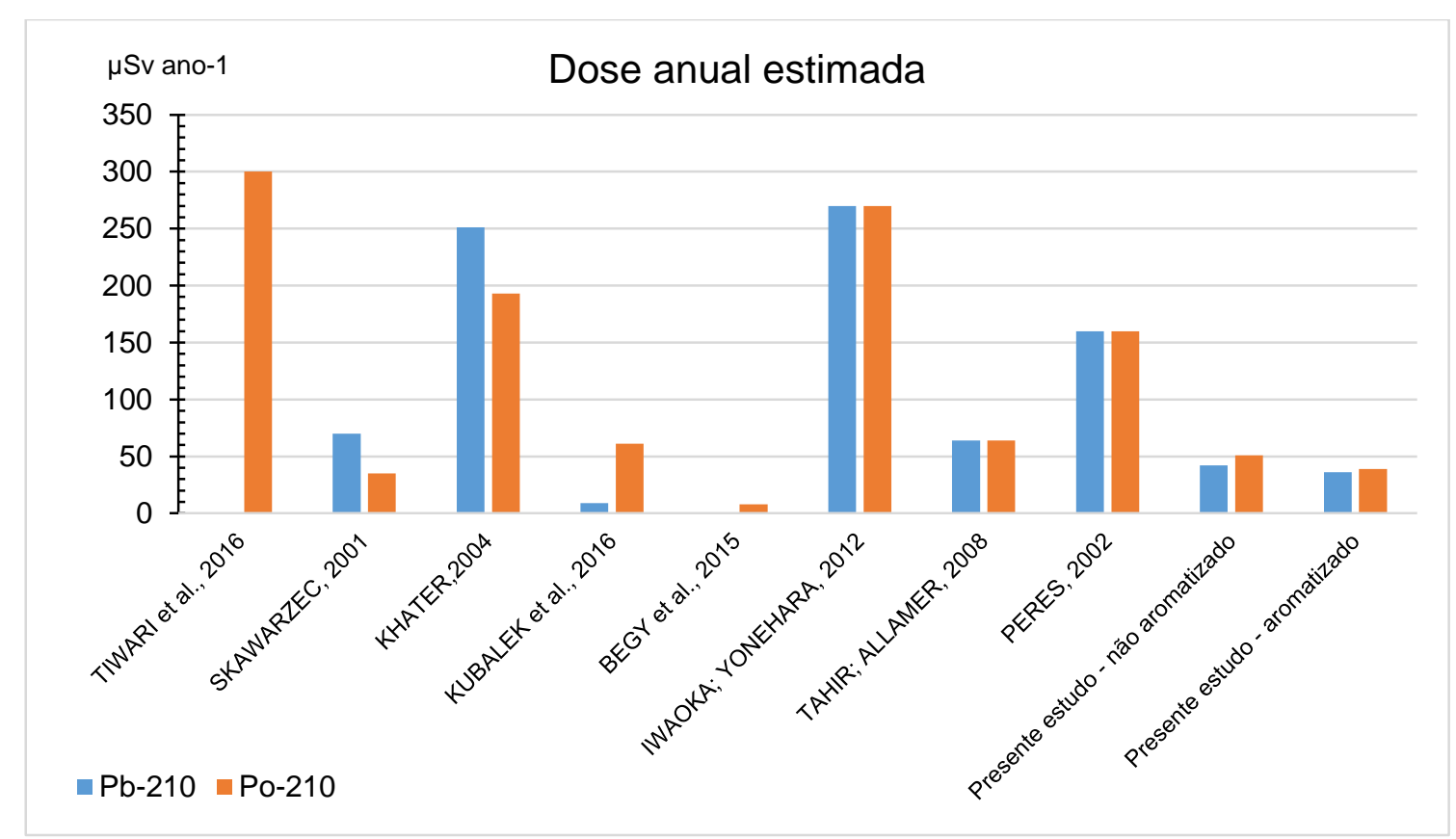

TABELA 4.27 - Dose anual estimada dos radionuclídeos ${ }^{210} \mathrm{~Pb}$ e ${ }^{210} \mathrm{Po}$ ( $\mu$ Sv ano-1) nos derivados de tabaco analisados e de artigos da literatura

\begin{tabular}{|c|c|c|c|}
\hline \multicolumn{4}{|c|}{ DOSE ANUAL ESTIMADA ( $\mu$ SV ANO $\left.{ }^{-1}\right)$} \\
\hline & \multirow[t]{2}{*}{ Derivado de tabaco } & \multicolumn{2}{|c|}{ Radionuclídeo } \\
\hline & & ${ }^{210} \mathrm{~Pb}$ & ${ }^{210} \mathrm{Po}$ \\
\hline $\begin{array}{c}\text { SAVIDOU ET AL., } \\
2006\end{array}$ & Folhas de tabaco & 163 & 124 \\
\hline \multirow{4}{*}{ PRESENTE ESTUDO } & Cigarro de palha & 60 & 46 \\
\hline & Charuto & 26 & 43 \\
\hline & Fumo de corda & 70 & 43 \\
\hline & Rapé & 54 & 67 \\
\hline
\end{tabular}




\subsubsection{Dose anual efetiva}

A dose anual efetiva foi determinada somando as doses estimadas dos radionuclídeos ${ }^{210} \mathrm{~Pb}$ e ${ }^{210} \mathrm{Po}$ nos grupos amostrados. Na FIG. 4.66 são apresentados os valores determinados no presente trabalho.

FIGURA 4.66 - Dose anual efetiva ( $\mu \mathrm{Sv}$ ano-1)

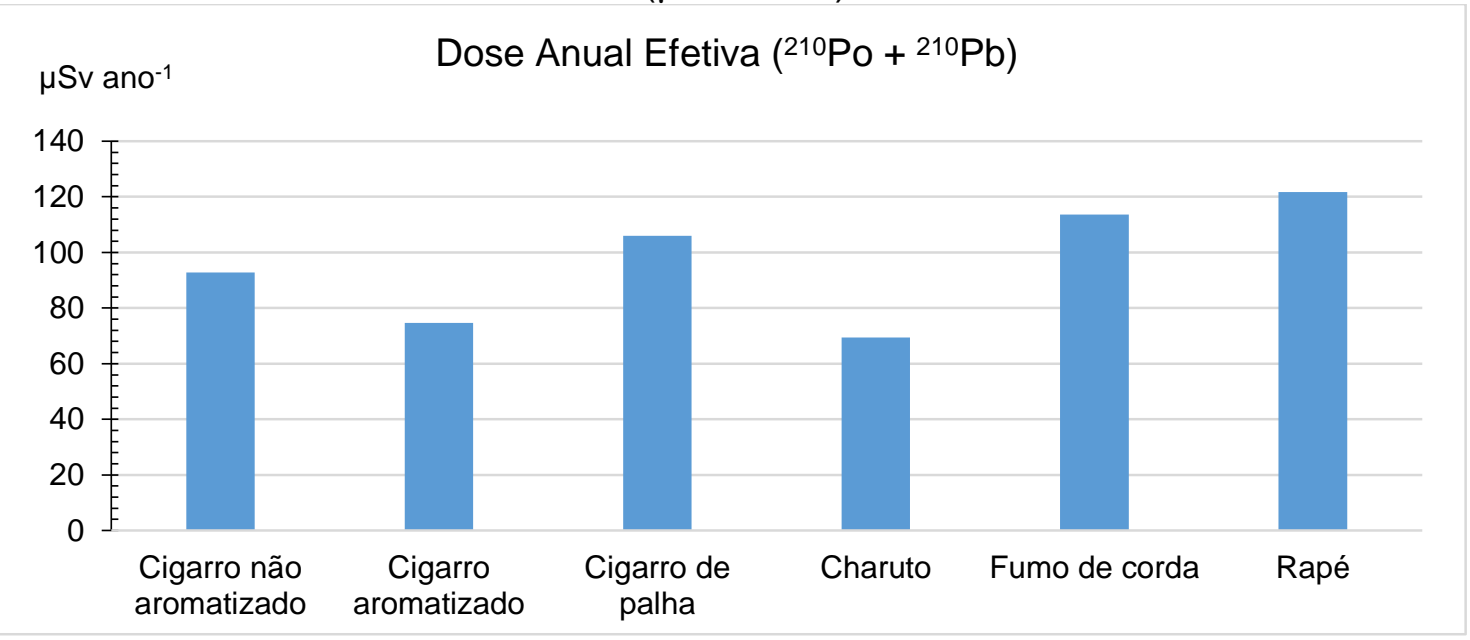

A dose anual efetiva para os derivados de tabaco estudados calculada variou de $69,5 \mu \mathrm{Sv}$ ano-1 a $121 \mu \mathrm{Sv}$ ano-1, valores abaixo da dose de inalação limite para o público estipulada pela International Commission on Radiological Protection (ICRP) de 1 mSv ano-1 (ICRP Publ. 82),

\subsection{Caracterização química inorgânica e radiológica dos derivados da Nicotiana tabacum L. estudados}

Nas TAB. $4.28,4.29$ e 4.30 são apresentados os intervalos de concentração para os elementos químicos e radionuclídeos naturais determinados nas respectivas técnicas, INAA, EDXRF e radioquímica para os derivados de tabaco analisados no presente trabalho.

$\mathrm{Na}$ técnica de INAA os elementos $\mathrm{Ca}$ e $\mathrm{K}$ apresentaram os maiores valores de concentração (\%) embora as concentrações de $\mathrm{K}$ ficaram abaixo do LID na metodologia em todas as amostras de cigarros aromatizados. 
Os elementos traços que apresentaram maiores valores de concentração foram: $\mathrm{Ba}, \mathrm{Br}, \mathrm{Rb}$ e $\mathrm{Zn}$, e os elementos terras raras que apresentaram maiores valores de concentração no foram Ce e La.

Observou-se que os maiores valores de concentração foram determinados nas amostras de cigarro de palha.

Pela técnica de INAA os valores de concentração ficaram abaixo do LID para os elementos $\mathrm{Sb}, \mathrm{Ta}, \mathrm{U}, \mathrm{Lu}$ e $\mathrm{Nd}$ e, para o elemento $\mathrm{K}$ nas amostras de cigarros aromatizados. O elemento Se somente foi determinado em cinco amostras (quatro fumos de corda e um rapé), o elemento Tb foi determinado em sete amostras (um cigarro não aromatizado, três cigarros de palha e três fumos de corda) e o elemento $\mathrm{Yb}$ foi determinado somente em duas amostras (um cigarro de palha e um fumo de corda)

Pode-se concluir que a técnica de INAA foi satisfatória para determinação dos elementos analisados. 
TABELA 4.28 - Intervalo de concentração dos elementos determinados pela técnica de INAA em $\mathrm{mg} \mathrm{kg}^{-1} \mathrm{e} * \%$, nos derivados de tabaco analisados

\begin{tabular}{|c|c|c|c|c|c|}
\hline & $\begin{array}{c}\text { Cig. não } \\
\text { aromatizado }\end{array}$ & $\begin{array}{c}\text { Cig. } \\
\text { aromatizado }\end{array}$ & $\begin{array}{c}\text { Cig. de } \\
\text { palha }\end{array}$ & Charuto & $\begin{array}{c}\text { Fumo de } \\
\text { corda }\end{array}$ \\
\hline${ }^{*}$ Ca & $2,3-3,8$ & $1,8-4,1$ & $1,9-6,6$ & $2,6-4,6$ & $1,1-4,6$ \\
\hline${ }^{*}$ Fe & $0,04-0,11$ & $0,04-0,11$ & $0,04-0,33$ & $0,05-0,06$ & $0,02-0,08$ \\
\hline${ }^{*} \mathbf{K}$ & $2,4-4,5$ & $<0,3$ & $0,8-5,3$ & $2,0-5,6$ & $1,1-5,4$ \\
\hline${ }^{*} \mathbf{N a}$ & $0,04-0,07$ & $0,04-0,12$ & $0,01-0,2$ & $0,04-0,10$ & $0,01-0,12$ \\
\hline $\mathbf{A s}$ & $1,4-2,9$ & $2,1-2,9$ & $1,9-6,4$ & $2,4-2,5$ & $1,5-3,2$ \\
\hline $\mathbf{B a}$ & $89-207$ & $82-165$ & $67-197$ & $74-165$ & $41-207$ \\
\hline $\mathbf{B r}$ & $7-87$ & $21-72$ & $30-153$ & $49-164$ & $22-226$ \\
\hline $\mathbf{C o}$ & $0,6-1,1$ & $0,5-1,1$ & $0,4-1,8$ & $0,3-0,6$ & $0,2-1,4$ \\
\hline $\mathbf{C r}$ & $1,1-4,4$ & $1,4-2,6$ & $0,8-8,1$ & $1,1-4,7$ & $1,1-5,2$ \\
\hline $\mathbf{C s}$ & $0,1-0,5$ & $0,1-0,4$ & $0,1-0,5$ & $0,1-0,2$ & $0,1-0,5$ \\
\hline $\mathbf{H f}$ & $0,1-0,3$ & $0,1-0,4$ & $0,1-1,2$ & $0,3-0,5$ & $0,1-0,6$ \\
\hline $\mathbf{R b}$ & $22-43$ & $21-33$ & $14-51$ & $19-43$ & $12-46$ \\
\hline $\mathbf{T h}$ & $<0,38$ & $<0,38$ & $0,4-1,6$ & $<0,38$ & $0,4-0,9$ \\
\hline $\mathbf{Z n}$ & $26-45$ & $31-43$ & $43-137$ & $36-80$ & $22-127$ \\
\hline $\mathbf{C e}$ & $1,1-5,7$ & $0,9-2,3$ & $2-11$ & $1,8-1,9$ & $1-25$ \\
\hline $\mathbf{E u}$ & $<0,09$ & $<0,09$ & $0,1-0,2$ & $<0,09$ & $0,1-0,2$ \\
\hline $\mathbf{L a}$ & $1,2-2,7$ & $1,4-3,8$ & $1-31$ & $<0,53-0,89$ & $1-72$ \\
\hline $\mathbf{S c}$ & $0,1-0,4$ & $0,2-0,3$ & $0,1-1,3$ & $0,09-0,73$ & $0,05-0,06$ \\
\hline $\mathbf{S m}$ & $0,08-0,23$ & $0,08-1,17$ & $0,12-0,84$ & $<0,04$ & $0,04-0,84$ \\
\hline & & & & & \\
\hline
\end{tabular}

Pela técnica de EDXRF foi possível determinar os valores de concentração dos elementos maiores $\mathrm{Al}, \mathrm{Cl}, \mathrm{I}, \mathrm{Mg}, \mathrm{Mn}, \mathrm{P}$ e $\mathrm{S}$, os valores de concentração dos elementos traços $\mathrm{Cd}, \mathrm{Cu}, \mathrm{Hg}, \mathrm{Ni}, \mathrm{Pb}, \mathrm{Sb}, \mathrm{Se}, \mathrm{Sr}$ e V e o elemento terra rara $\mathrm{Nd}$. Estes elementos não foram determinados pela técnica de INAA ou apresentaram valores de concentração abaixo do LID no método. 
TABELA 4.29 - intervalo de concentração dos elementos determinados pela técnica de EDXRF em mg kg-1 $\mathrm{e}^{* \%} \%$, nos derivados de tabaco analisados

\begin{tabular}{|c|c|c|c|c|c|}
\hline & $\begin{array}{c}\text { Cig. não } \\
\text { aromatizado }\end{array}$ & $\begin{array}{c}\text { Cig. } \\
\text { aromatizado }\end{array}$ & Cig. de palha & Charuto & $\begin{array}{c}\text { Fumo de } \\
\text { corda }\end{array}$ \\
\hline${ }^{*} \mathrm{Al}$ & $0,04-0,09$ & $0,05-0,09$ & $0,05-0,25$ & $0,06-0,12$ & $0,01-0,17$ \\
\hline${ }^{\star} \mathrm{Ca}$ & $1,5-1,8$ & $1,6-1,8$ & $1,3-3,4$ & $1,6-2,4$ & $1,4-2,4$ \\
\hline${ }^{*} \mathrm{Cl}$ & $0,5-1,3$ & $0,6-1,1$ & $0,12-1,3$ & $0,7-1,5$ & $0,2-17$ \\
\hline${ }^{*} \mathrm{Fe}$ & $0,04-0,09$ & $0,05-0,08$ & $0,03-0,20$ & $0,04-0,05$ & $0,03-0,12$ \\
\hline *I & $0,019-0,022$ & $0,020-0,022$ & $0,016-0,024$ & $0,019-0,021$ & $0,016-0,023$ \\
\hline${ }^{*} \mathrm{~K}$ & $2,0-3,5$ & $2,4-3,3$ & $0,9-3,6$ & $2,6-5,5$ & $1,1-4.6$ \\
\hline${ }^{*} \mathrm{Mg}$ & $0,5-1,2$ & $0,7-1,1$ & $0,3-1,3$ & $1,4-2,4$ & $0,1-2,2$ \\
\hline${ }^{*} \mathrm{Mn}$ & $0,01-0,03$ & $0,01-0,02$ & $0,01-0,08$ & $0,01-0,02$ & $0,01-0,08$ \\
\hline${ }^{\star} \mathbf{P}$ & $0,1-0,2$ & $0,1-0,2$ & $0,1-0,2$ & $0,2-0,3$ & $0,1-0,5$ \\
\hline${ }^{*} S$ & $0,2-0,3$ & $0,2-0,3$ & $0,2-0,4$ & $0,4-0,7$ & $0,03-0,51$ \\
\hline As & $0,061-0,065$ & $0,060-0,063$ & $0,06-0,07$ & $0,06-0,07$ & $0,06-0,07$ \\
\hline $\mathrm{Br}$ & $39-77$ & $38-74$ & $18-75$ & $38-98$ & $17-257$ \\
\hline Cd & $0,2-1,2$ & $0,4-1,1$ & $0,4-2,7$ & $0,4-1,3$ & $1,5-4,0$ \\
\hline Co & $0,5-1,2$ & $0,4-1,1$ & $0,5-1,3$ & $0,3-0,8$ & $0,1-1,4$ \\
\hline $\mathrm{Cr}$ & $0,8-2,5$ & $0,9-1,1$ & $0,8-1,8$ & $0,9-1,3$ & $0,8-1,2$ \\
\hline Cs & $0,04-0,05$ & $0,04-0,05$ & $0,04-0,07$ & $0,046-0,054$ & $0,04-0,06$ \\
\hline $\mathrm{Cu}$ & $4,4-6,0$ & $4,4-5,3$ & $4,1-6,0$ & $4,9-6,2$ & $4,3-7,9$ \\
\hline $\mathbf{H g}$ & $0,020,03$ & $0,027-0,032$ & $0,01-0,03$ & $0,02-0,03$ & $0,02-1,3$ \\
\hline $\mathbf{N i}$ & $0,7-2,2$ & $0,8-1,5$ & $1,0-2,7$ & $0,9-1,4$ & $0,6-1,9$ \\
\hline $\mathrm{Pb}$ & $0,3-1,5$ & $0,4-1,7$ & $0,4-1,8$ & $0,4-1,8$ & $0,8-1,6$ \\
\hline $\mathbf{R b}$ & $19-21$ & $19-20$ & $15-21$ & $18-20$ & $19-21$ \\
\hline $\mathbf{S b}$ & $0,02-0,03$ & $0,021-0,024$ & $0,02-0,04$ & $0,02-0,03$ & $0,02-0,03$ \\
\hline $\mathrm{Se}$ & $0,039-0,045$ & $0,042-0,044$ & $0,041-0,047$ & $0,043-0,045$ & $0,043-0,048$ \\
\hline $\mathrm{Sr}$ & $80-131$ & $88-128$ & $64-181$ & $113-167$ & $65-188$ \\
\hline Th & $0,02-0,09$ & $0,05-0,08$ & $0,05-0,12$ & $0,04-0,08$ & $0,04-0,13$ \\
\hline $\mathbf{U}$ & $0,1-0,2$ & $0,1-0,3$ & $0,1-0,3$ & $0,1-0,3$ & $0,1-0,4$ \\
\hline V & $0,8-2,1$ & $0,7-1,7$ & $0,7-3,7$ & $0,7-1,1$ & $0,4-2,4$ \\
\hline Zn & $16-23$ & $9-20$ & $17-25$ & $21-25$ & $18-28$ \\
\hline La & $2-16$ & $3-9$ & $9-39$ & $6-13$ & $2-27$ \\
\hline Nd & $6-18$ & $5-6$ & $5-8$ & $<5,02-6$ & $6-13$ \\
\hline Sm & $1,1-2,3$ & $0,9-1,8$ & $1,3-3,3$ & $1,5-2,1$ & $1,0-5,4$ \\
\hline
\end{tabular}

$\mathrm{Na}$ técnica de EDXRF os elementos $\mathrm{Ca}$ e $\mathrm{K}$ apresentaram os maiores valores de concentração (\%). A concentração do elemento $\mathrm{K}$ nos cigarros aromatizados apresentaram concentrações detectáveis e com valores semelhantes as determinadas nas amostras de cigarros não aromatizados. 
Alguns elementos como o As, Cs, Hg, Sb Se e U apresentaram valores de concentrações praticamente constantes em todas as amostras analisadas.

Os grupos que apresentaram maiores valores de concentração determinados para os elementos analisados foram os cigarros de palha e os fumos de corda.

Pode concluir que a técnica de EDXRF foi satisfatória para determinação dos elementos analisados e pode ser usada para complementar a técnica de INAA na caracterização elementar dos derivados da Nicotiana tabacum L. com a determinação de um maior número de elementos.

O radionuclídeo que apresentou maiores valores de concentração foi o ${ }^{228} \mathrm{Ra}$, os valores de concentração do radionuclídeo ${ }^{232} \mathrm{Th}$ foram praticamente constantes em toda amostragem e o grupo de amostras que apresentaram os maiores valores de concentração foi o de fumo de corda.

A determinação da concentração de atividade dos radionuclídeos naturais é importante para caracterizar radiologicamente os derivados da Nicotiana tabacum L. e para calcular as doses estimadas de radiação inalada durante o consumo destes derivados.

TABELA 4.30 - Intervalo de concentração dos radionuclídeos naturais em $\mathrm{mBq} \mathrm{g}^{-1}$, nos derivados de tabaco analisados

\begin{tabular}{c|c|c|c|c|c}
\hline & $\begin{array}{c}\text { Cig. não } \\
\text { aromatizado }\end{array}$ & $\begin{array}{c}\text { Cig. } \\
\text { aromatizado }\end{array}$ & $\begin{array}{c}\text { Cig. de } \\
\text { palha }\end{array}$ & Charuto & $\begin{array}{c}\text { Fumo de } \\
\text { corda }\end{array}$ \\
\hline${ }^{\mathbf{2 3}} \mathbf{U}$ & $1,3-2,4$ & $1,8-3,1$ & $1,6-4,1$ & $1,6-3,4$ & $1,9-4,6$ \\
\hline${ }^{226} \mathbf{R a}$ & $7-13$ & $5-14$ & $6-22$ & $18-28$ & $6-40$ \\
\hline${ }^{\mathbf{2 1 0}} \mathbf{P b}$ & $15-26$ & $<4,9-25$ & $17-55$ & $6-22$ & $5-78$ \\
\hline${ }^{\mathbf{2 1 0}} \mathbf{P o}$ & $14-25$ & $6-22$ & $11-35$ & $15-22$ & $10-28$ \\
\hline${ }^{232} \mathbf{T h}$ & $0,1-0,4$ & $0,2-0,3$ & $0,1-0,5$ & $0,2-0,3$ & $0,1-0,5$ \\
\hline${ }^{228} \mathbf{R a}$ & $34-45$ & $26-69$ & $37-81$ & $52-78$ & $28-152$ \\
\hline
\end{tabular}




\section{CONCLUSÃO}

Embora existam muitos trabalhos sobre a composição química do cigarro estudar a composição de outros derivados da Nicotina tabacum L. produzidos e consumidos no Brasil é de extrema relevância, uma vez que um terço da população brasileira é tabagista e o país é o segundo maior produtor e o maior exportador do insumo.

O presente trabalho determinou a concentração dos radionuclídeos naturais ${ }^{238} \mathrm{U},{ }^{226} \mathrm{Ra},{ }^{210} \mathrm{~Pb}$ e ${ }^{210} \mathrm{Po}$ da série de decaimento do ${ }^{238} \mathrm{U} ;{ }^{232} \mathrm{Th}$ e ${ }^{228} \mathrm{Ra}$ da série de decaimento do ${ }^{232} \mathrm{Th}$ e a concentração de elementos maiores e traços As, $\mathrm{Ba}, \mathrm{Ca}, \mathrm{Ce}, \mathrm{Co}, \mathrm{Cr}, \mathrm{Cs}$, Eu, Fe, Hf, K, La, Lu, Nd, Rb, Sb, Sc, Se, Sm, Ta, Tb, Th, U, $\mathrm{Yb}$ e $\mathrm{Zn}$ em derivados de tabaco produzidos e comercializados no Brasil usando as técnicas analíticas alfa e beta total após separação radioquímica, espectrometria alfa após separação radioquímica, análise por ativação com nêutrons instrumental - INAA e Fluorescência de Raios X - EDXRF com o objetivo de determinar as concentrações dos radionuclídeos naturais e composição elementar de amostras de cigarro não aromatizados, cigarro aromatizado, rapé, cigarro de palha e fumo de corda (rolo e desfiado; natural e aromatizado), todos adquiridos em tabacarias e comércios públicos nacionais.

As técnicas analíticas empregadas se mostraram bastante satisfatórias e sensíveis para a determinação da maioria dos elementos estudados neste trabalho.

Entre os elementos maiores determinados, os maiores valores de concentração foram obtidos para os elementos $\mathrm{Ca}$ e $\mathrm{K}$ e entre os elementos traço, os maiores valores de concentração foram obtidos para os elementos $\mathrm{Ba}, \mathrm{Br}, \mathrm{Ce}, \mathrm{Cu}$, $\mathrm{Nd}, \mathrm{b}, \mathrm{Sr}$ e Zn na maioria das amostras.

Os radionuclídeos que apresentaram maiores concentrações em todas as amostras estudadas foram ${ }^{228} \mathrm{Ra},{ }^{210} \mathrm{~Pb}$ e ${ }^{210} \mathrm{Po}$ e as menores concentrações foram obtidas para os radionuclídeos ${ }^{238} \mathrm{U}$ e ${ }^{232} \mathrm{Th}$.

O grupo de amostras que apresentaram as maiores concentrações, tanto para os elementos maiores e traço como para os radionuclídeos naturais foram os de cigarros de palha e fumos de corda. 
As maiores doses médias estimadas e doses efetivas anuais foram obtidas para os grupos de amostras de cigarro de palha, fumo de corda e rapé.

Os resultados obtidos da concentração dos elementos traco, maiores e radiocnuclídeos naturais nas amostras de cigarros não aromatizados, cigarros aromatizados, cigarros de palha, charutos, fumos de corda e rapés permitiram a realização da caracterização química inorgânica e caracterização radiológica dos principais derivados da Nicotiana tabacum $L$ consumidos no Brasil e contribuíram para o estabelecimento de um banco de dados sobre a caracterização radiológica e química inorgânica. 


\section{SUGESTÕES PARA TRABALHOS FUTUROS}

Este trabalho teve por objetivo caracterizar radiologicamente $\mathrm{e}$ elementarmente produtos derivados da Nicotiana tabacum L.de origem nacional. Sugere-se para trabalhos futuros o estudo dos radionuclídeos e elementos químicos determinados no presente trabalho no ser humano e também em produtos importados derivados da Nicotiana tabacum $L$ consumidos no Brasil. 


\section{REFERÊNCIAS BIBLIOGRÁFICAS}

A.C.CAMARGO, Tudo sobre o câncer de pulmão. http://www.accamargo.org.br/tudo-sobrecancer/pulmao/33/?gclid=CJzcsZiQjtYCFccFkQoddZkl9g Acessado em 10/11/2016.

ABD EL-AZIZ, N.; KHATER, A.E.M.; AL-SEWAIDAN, H.A. Natural radioactivity contents in tobacco. International Congress Series, v. 1276, 407-408, 2005.

ABD EL-SAMAD, M.; HANAFI, H.A. Analysis of toxic heavy metals in cigarettes by Instrumental Neutron Activation Analysis. Journal Taibah University for Science, v.11, p.822-829, 2017.

ALENCAR, M. M. Determinação de isótopos de urânio e tório em amostras de liquens canoparmelia texana. Dissertação (Mestrado em Tecnologia Nuclear Aplicações) - Instituto de pesquisas Energéticas e Nucleares, Universidade de São Paulo, São Paulo, 2008.

ATHALYE, V. V.; MISTRY, K.B. Foliar retention, transport and leaching of 210-Po and 210Pb. Radiation botany. V.12, 287-290,1972.

AVELAR, L. E. B. A moderação em excesso: estudo sobre a história das bebidas na sociedade colonial. Tese (Doutorado) - Universidade de São Paulo, São Paulo, 2010.

BEGY, R.C.; SIMON, H.; KELEM, S., ${ }^{210}$ Po inhalation due to smoking: a dose estimation. Journal of Radioanal Nuclear Chemistry. v.306, p.257-261, 2015.

BODE, P. Instrumental and organizational aspects of a neutron activation analysis laboratory. Delft, Netherlands: Interfaculty Reactor Institute, p.147, 1996.

BONA, I.A.T.; SARKIS, J.E.S; SALVADOR, V.L.R.; SOARES, A.L.R.; KLAMT, S.C. Analise Arqueométrica de Ceramica Tupiguarani da Região Central do Estado do Rio Grande do Sul, Brasil, Usando Fluorescencia de Raios X por Dispersão de Energia (EDXRF). Quimica Nova. v.30, 785-790, 2007.

BONATO, A.A. A fumicultura no Brasil e a convenção-quadro para controle do tabaco. DESER - Departamento de Estudos Socioeconômicos Rurais, Curitiba, 2006. 
CAETANO, A. F. P. Entre a sombra e o sol-a revolta da cachaça, a freguesia de São Gonçalo de Amarante e a crise política fluminense (Rio de Janeiro, 16401667). - Dissertação (Mestrado) Universidade Federal Fluminense, Niteroi, 2003.

CHAUHAN, P.; CHAUHAN, R.P. Measurement of fertilizers induced radioactivity in tobacco plants and elemental analysis using ICAP e AES. Radiation Measurements. v. 63, 2014.

DAMATTO, S. R. Radionuclídeos naturais das séries do ${ }^{238} \mathrm{U}$ e ${ }^{232} \mathrm{Th}$, elementos traço e maiores determinados em perfis de sedimento da Baixada Santista para avaliação de áreas impactadas. Tese (Doutorado). Instituto de Pesquisas Energéticas e Nucleares, São Paulo, 2010.

ELDRIDGE A.; BETSON T. R.; GAMA, M. V.; MCADAMA, K. Variation in tobacco and mainstream smoke toxicant yields from selected commercial cigarette products. Regulatory Toxicology and Pharmacology, v. 71, p.409-427, 2015.

EXLEY, C.; Begum, A.; Woolley, M. P.; Bloor, R.N. Aluminum in Tobacco and Cannabis and Smoking-Related Disease. The American Journal of Medicine, v.119, 2006.

FRANCISCONI, L.S.; DAMATTO, S. R.; SILVA, P. S. C. Determination of As, Se and $\mathrm{Sb}$ in different trades and blends of tobacco by neutron activation analysis. International Nuclear Atlantic Conference - INAC, Belo Horizonte, 2011.

GODOY, J.H.; GOUVEA D.R.; AZEREDO, A.M.G. ${ }^{226} \mathbf{R a} /{ }^{210} \mathbf{P b} /{ }^{210} \mathrm{Po}$ Equilibrium in Tobacco Leaves. Radiation Protection Dosimetry, v.45, p 299-300, 1992.

HARRIS. D. S.; ANTHENELLI. R. M. Current Psychiatry Reports. 344-351, 2005.

HECHT, S. S.; Lung Carcinogenesis by tobacco smoke. International journal of cancer, v. 131, n. 12, 2724-2732, 2012.

IBGE, INSTITUTO BRASILEIRO DE GEOGRAFIA E ESTATísticA. Pesquisa Nacional de Saúde, 2013.2 Disponível em: http://biblioteca.ibge.gov.br/visualizacao/livros/liv91110.pdf. Acessado em 10/10/2016. 
INCA, INSTITUTO NACIONAL DO CÂNCER. O controle do Tabagismo no Brasil. Disponível em: www.inca.gov.br/prevenção/tabagismo/controle_br.html. Acessado em 01/08/2016.

INCA, INSTITUTO NACIONAL DO CÂNCER. Aditivos em cigarros- notas Técnicas para Controle do Tabagismo. Rio de Janeiro, 2011.

INCA, INSTITUTO NACIONAL DO CÂNCER. Observatório da Política Nacional de Controle do Tabaco. Disponível em: http://www2.inca.gov.br/wps/wcm/connect/observatorio_controle_tabaco/site/home/d ados_numeros/prevalencia-de-tabagismo. Acessado em janeiro de 2017.

ICRP, INTERNATIONAL COMMISSION ON RADIOLOGICAL PROTECTION. Publication 119, "Compendium of Dose Coefficients Based on ICRP Publication 60, v.42, 2013.

ICRP, INTERNATIONAL COMMISSION ON RADIOLOGICAL PROTECTION. Publication 82, Protection of the public in situations of prolonged radiation exposure v.42, 1999.

IVANOVICH, M. E HARMON, R. S Uranium-series disequilibrium: applications to Earth, Marine and Environmental Sciences. Claredon Press - Oxford, 1992.

KABATA-PENDIAS, A. Trace elements in soils and plants. CRC press, 2010.

KARAGUEUZIAN HS1, WHITE C, SAYRE J, NORMAN A., Cigarette smoke radioactivity and lung cancer risk. Journal of the Society for Research on Nicotine and Tobacco, v. 14, p.79-90, 2012.

KAZI, T. G., JALBANI, N.; ARAINA, M. B.; JAMALIA, M. K.; AFRIDI, H. I.; SARFRAZA, R. A.; SHAHA, A. Q. Toxic metals distribution in different components of Pakistani and imported cigarettes by electrothermal atomic absorption spectrometer. Journal of Hazardous Materials, v.163, p.302-307, 2009.

KELECON, A.; GOUVEIA, R.C.S.; SANTOS, P.L. Levels of ${ }^{210} \mathrm{Po}$ and ${ }^{210} \mathrm{~Pb}$ in cigars. Journal of Radioanalytical and Nuclear Chemistry, v. 253, N. 1, $129-133$, 2002.

KHATER, A. E. M.Polonium-210 budget in cigarettes. J. Environ. Radioactivity, v.7, p 33-41, 2004. 
KILTHAU, G. F. Cancer risk in relation to radioactivity in tobacco. Radiologic Technology. v. 67, n. 3, 1996.

KUBALEK, D.; SERSAS, G.; STROK, M; BENEDIK, L.; JERAN, Z Radioactivity of cigarettes and the importance of 210Po and thorium isotopes for radiation dose assessment due to smoking. Journal of Environmental Radioactivity. $155-156$, 2016.

LEFFINGWELL, J.C. Chemistry BA Basic Chemical Constituents of Tobacco Leaf and Differences among Tobacco Types. Disponível em: http://www.leffingwell.com/download/Leffingwell\%20\%20Tobacco\%20production $\% 20$ chemistry\%20and\%20technology.pdf. Acessado em setembro de 2016.

MARQUES, C. À Margem da Economia: cachaça e protocampesinato negro no litoral sul fluminense (1800-1888). Dissertação (Doutorado). Universidade Federal Fluminense, Niterói, 2011.

MARTINEZ, T.; LARTIGUE, J.; ZARAZUA, G.; AVILA-PEREZ, P.; NAVARRETE, M.; TEJEDA, S. Aplication of the Total Reflection X-ray Fluorescence technique to trace elements determination in tobacco. Spectrochimica Acta Part B, v.63, p.1469-1472, 2008.

MELLO Jr, F. M., KOCKEL, M. F. Aspectos do tabaco e do cauim no Brasil quinhentista. Disponível em: http://www.anpuhsp.org.br/sp/downloads/CD\%20XX\%20Encontro/PDF/Pain\%E9is/ Marcelo\%20Fidelis\%20Kockel.pdf. Acessado em novembro de 2016.

MILNEROWICZ, H.; SCISKALSKA, M., DUL, M. Pro-inflammatory effects of metals in persons and animals exposed to tobacco smoke. Journal of Trace Elements in Medicine and Biology, v.29, p.1-10, 2015.

MOHAPATRA, P.; PREET, R.; DAS, D. SATAPATHY, S.R.; SIDDHARTH, S. CHOUDHURI, T.; WYATT, M. D.; KUNDU, C. N. The contribution of heavy metals in cigarette smoke condensate to malignant transformation of breast epithelial cells and in vivo initiation of neoplasia through induction of a PI3K-AKT-NFkB cascade. Toxicology and applied Pharmacology, v.274, p. 168-179, 2014.

MOREIRA, S. R.D. Determinação de ${ }^{210} \mathrm{~Pb}$ em aguas minerais da cidade de Aguas da Prata. Dissertação (Mestrado) - Instituto de Pesquisas Energéticas e Nucleares IPEN/CNEN-SP, São Paulo,1993. 
MÜLLER, A. L. H; BIZZI, C. A. PEREIRA, J. S. F.; MESKO, M. F.; MORAES,D. P.; FLORESA, E. M. M.; MULLER, E. I. Bromine and Chlorine Determination in Cigarette Tobacco using Microwave-Induced Combustion and Inductively Coupled Plasma Optical Emission Spectrometry. J. Braz. Chem. Soc., v. 22, n. 9, p.1649-1655, 2011.

NIERI NETO, A. Determinação de ${ }^{210} \mathrm{~Pb}$ e ${ }^{210} \mathrm{Po}$ em águas minerais radioativas. Disertação (Mestrado). Instituto de Pesquisas Energéticas e Nucleares, São Paulo, 1996.

OLIVEIRA, J. Determinação de ${ }^{226} \mathrm{Ra}$ e ${ }^{228} \mathrm{Ra}$ em aguas minerais da região de Aguas da Prata. Dissertação (Mestrado) - Instituto de Pesquisas Energéticas e Nucleares - IPEN/CNEN-SP, São Paulo,1993.

ORTEC INTERWINNERTM 6,0 MCA Emulation, Data and Analisys software for gamma and alpha spectroscopy IW B-32. Oak Ridge, TN, USA, 2004.

PANTA, Y. M.; QIAN, S.; CROSS, C. CIZDZIEL, J. V. Mercury content of whole cigarettes, cigars and chewing tobacco packets using pyrolysis atomic absorption spectrometry with gold amalgamation

PAPPAS, R. S. Ph.D. Toxic Elements in Tobacco and in Cigarette Smoke: Inflammation and Sensitization. Metallomics, v 3, p.1181-1198, 2011.

PERES, A.C. Determinação de ${ }^{210} \mathrm{~Pb}$ e ${ }^{210} \mathrm{Po}$ em tabaco de cigarros nacionais. Dissertação (Mestrado), Instituto de Pesquisas Energéticas e Nucleares IPEN/CNEN-SP, São Paulo,1999.

PERES, A.C.; HIROMOTO, G., Evaluation of ${ }^{210} \mathrm{~Pb}$ and ${ }^{210} \mathrm{Po}$ in cigarette tobacco produced in Brazil. Journal of Environmental Radioactivity, v. 62, p.115-119, 2002.

PIADÉ, J. J.; JACCARDB, G.; DOLKAA, C.; BELUSHKINA, M.; WAJROCK, S. Differences in cadmium transfer from tobacco to cigarettesmoke, compared to arsenic or lead. Toxicology Reports, v. 2, p.12-26, 2015.

PINTO, M,; UGÁ, M, A, D, Custo do tratamento de pacientes com histórico de tabagismo em hospital especializado em câncer, Revista Saúde Pública, v.45, p. 575-578, 2011.

PRATA, V. M.; EMÍDIO, E. S.; DÓREA, H. D.; Aplicação de métodos quimiométricos na caracterização de charutos utilizando extração por dispersão da matriz em fase sólida e análise por cromatografia gasosa acoplada à espectrometria de massas. Química Nova, v. 34, n. 1, p.53-58, 2011. 
RAMOS, G. F. Determinação de isótopos de urânio, tório e polônio em perfis de sedimentos da Baixada Santista, SP. (Mestrado em Tecnologia Nuclear Aplicações) - Instituto de Pesquisas Energéticas e Nucleares, Universidade de São Paulo, São Paulo, 2010.

RICKERT, W. S.; JOZA, P. J.; TRIVED, A. H.; MOMIM, R. A.; WAGSTAFF, W. G.; LAUTERBACH, J. H. Chemical and toxicological characterization of commercial smokeless tobacco products available on the Canadian market. Reg. Toxic. Pharmac. v. 53, 121-133, 2009.

SAKODA, A.; FUKAO, K.; KAWABE, A.; KATAOKA, T., HANAMOTO, K.; YAMAOKA, $\mathrm{K}$; Radioactivity of ${ }^{210} \mathrm{~Pb}$ in Japanese cigarettes and radiation dose from smoking inhalation. Radiation Protection Dosimetry. v.150, 109 - 113, 2012.

SAVIDOU, A.; KEHAGIA, K.; ELEFTHERIADIS, K., Concentration levels of ${ }^{210} \mathrm{~Pb}$ and ${ }^{210} \mathrm{Po}$ in dry tobacco leaves in Greece. Journal of Environmental Radioactivity, v. 8, p.94-102, 2006.

SHOUSHA, H. A.; AHMAD, F., Natural radioactivity in tobacco and radiation dose induced from smoking. Radiation Protection Dosimetry. v. 150, n.1, p. 91-95, 2012.

SILVA, C. F. Avaliação da concentração dos radionuclídeos naturais das series do ${ }^{238} \mathrm{U}$ e ${ }^{232} \mathrm{Th}$ nas variedades Burley e Virginia da Nicotina Tabacum $\mathrm{L}$. Dissertação (Mestrado). Instituto de Pesquisas Energéticas e Nucleares, São Paulo, 2015.

SILVA, L. C. C.; de Araújo, A. J.; de Queiroz, A. M. D.; Sales, M. P. U; Castellano, M.V.C.O. Controle do tabagismo: desafios e conquistas. Jornal Brasileiro de Pneumologia. n.42, 2016.

SILVA, P. S. C., FRANCISCONI, L.S.; MAIHARA, V.A. Concentração de elementos tóxicos $\mathrm{Cd}, \mathrm{Pb}$ e $\mathrm{Hg}$ em diferentes marcas e misturas de cigarros. CONGRESSO BRASILEIRO DE GEOQUIMICA, Diamantina, 2013.

SILVEIRA, R. L., DORNELLES, M., e FERRARI, S. (2012). Expansão da cultura do tabaco no sul do Brasil (1996-2006): características, mudanças e persistências na produção de tabaco e nos usos do território. Revista Bibliográfica de Geografía y Ciencias Sociales, Barcelona, 2012. 
Sinditabaco, Sindicato Interestadual da Indústria do Tabaco. Disponível em: http://www.sinditabaco.com.br Acesso em julho de 2016.

SKAWARZEC, B.; ULATOWSKI, J.; STRUMINSKA, D. I.; BORYŁO, A., Inhalation of ${ }^{210} \mathrm{Po}$ and ${ }^{210} \mathrm{~Pb}$ from cigarette smoking in Poland. Journal of Environmental Radioactivity, v.57, p.221-230, 2001.

SOUZA CRUZ. História do tabaco. Disponível em: http://www.souzacruz.com.br/group/sites/SOU_7UVF24.nsf/vwPagesWebLive/DO7V 9KPU/opendocument. Acessado em outubro de 2016.

TAHIR, S. N. A.; ALAAMER, A. S. ${ }^{210} \mathrm{~Pb}$ Concentrations in Cigarettes Tobaccos and Radiation Doses to the Smokers, Radiation Protection Dosimetry, v.130,.p.389391, 2008.

TALIO, M, C.; LUCONI, M, O. FERNANDEZ, Determination of nickel in cigarettes smoke by molecular fluorescence. Microchemical Journal. v. 99, p.486-491, 2011.

TIWARI, M.; SAHU,S. K.; BHANGARE, R. C.; PANDIT, G. G., Polonium in size fractionated mainstream cigarette smoke, predicted deposition and associated internal radiation dose. Journal of Environmental Radioactivity, p.162-163, 2016.

TRICHOPOULOS D, MOLLO F, TOMATIS L, AGAPITOS E, DELSEDIME L, ZAVITSANOS X, KALANDIDI A, KATSOUYANNI K, RIBOLI E, SARACCI R. Active and Passive Smoking and Pathological Indicators of Lung Cancer Risk in an Autopsy Study. JAMA. n. 268, p.1697-1701, 1992.

VIEGAS, C. A. A.; Formas não habituais de uso do tabaco. Jornal Brasileiro de Pneumologia. v. 34, n. 12, p.1069-1073, 2008.

WHO, World Health Organization. WHO report finds dramatic increase in life-saving tobacco control policies in last decade. Disponível em http://www.who.int/mediacentre/news/releases/2017/tobacco-report/en/. Acessado em março de 2017.

WÜNSCH FILHO, V.; MIRRA, A. P.; LÓPEZ, R.V.M. ANTUNES, L.F. Tabagismo e câncer no Brasil: evidências e perspectivas. Revista Brasileira de Epidemiologia, v. 13, p.175-187, 2010. 
YAPRAK, G., UYSAL, B.; Determination of low level ${ }^{210} \mathrm{~Pb}$ in tobacco. Journal of Radioanalytical and Nuclear Chemistry, v. 229, p. 153 -155, 1998.

ZAGÀ, V.; LYGIDAKIS, C.; CHAOUACHI, K. GATTAVECCHIA, E., Polonium and Lung Cancer. Journal of Oncology, 2011. 
APÊNDICE A.1 - Concentração dos elementos em mg kg-1 nos cigarros não aromatizados determinados por Analise por Ativação com Nêutrons Instrumental.

\begin{tabular}{|c|c|c|c|c|c|c|c|c|c|c|c|c|c|}
\hline & As & $\mathrm{Ba}$ & $\mathrm{Br}$ & $\mathrm{Ca}$ & $\mathrm{Ce}$ & Co & $\mathrm{Cr}$ & Cs & $\mathrm{Eu}$ & $\mathrm{Fe}$ & $\mathrm{Hf}$ & $\mathrm{K}$ & $\mathrm{La}$ \\
\hline CCDLA & $2,6 \pm 0,2$ & $128 \pm 16$ & $75,4 \pm 0,4$ & $27866 \pm 2006$ & $1,7 \pm 0,1$ & $0,86 \pm 0,04$ & $1,9 \pm 0,1$ & $0,11 \pm 0,02$ & $<0,09$ & $750 \pm 24$ & $0,15 \pm 0,01$ & $39179 \pm 2946$ & $1,50 \pm 0,04$ \\
\hline CCDLV & $<1,4$ & $146 \pm 18$ & $70,3 \pm 0,3$ & $32162 \pm 2205$ & $1,1 \pm 0,1$ & $0,79 \pm 0,03$ & $1,7 \pm 0,1$ & $0,13 \pm 0,03$ & $<0,09$ & $671 \pm 22$ & $0,18 \pm 0,02$ & $40442 \pm 3030$ & $1,22 \pm 0,03$ \\
\hline CCLMA & $<1,4$ & $183 \pm 22$ & $55,1 \pm 0,3$ & $38077 \pm 2521$ & $2,4 \pm 0,2$ & $0,98 \pm 0,04$ & $1,4 \pm 0,1$ & $0,13 \pm 0,02$ & $<0,09$ & $775 \pm 24$ & $0,24 \pm 0,02$ & $37908 \pm 2843$ & $1,62 \pm 0,04$ \\
\hline CCMG & $1,4 \pm 0,1$ & $13 \pm 2$ & $53,8 \pm 0,3$ & $33508 \pm 2304$ & $2,0 \pm 0,2$ & $0,84 \pm 0,03$ & $2,9 \pm 0,2$ & $0,15 \pm 0,03$ & $<0,09$ & $716 \pm 23$ & $0,25 \pm 0,02$ & $33459 \pm 2521$ & $1,66 \pm 0,04$ \\
\hline CCMV & $2,9 \pm 0,2$ & $153 \pm 18$ & $59,3 \pm 0,3$ & $37891 \pm 2486$ & $1,8 \pm 0,2$ & $0,99 \pm 0,04$ & $2,7 \pm 0,2$ & $0,17 \pm 0,03$ & $<0,09$ & $773 \pm 24$ & $0,23 \pm 0,02$ & $37405 \pm 2813$ & $1,76 \pm 0,04$ \\
\hline CCPZ & $1,7 \pm 0,1$ & $207 \pm 25$ & $75,7 \pm 0,3$ & $31392 \pm 2202$ & $2,2 \pm 0,2$ & $1,08 \pm 0,04$ & $2,2 \pm 0,2$ & $0,16 \pm 0,03$ & $<0,09$ & $1098 \pm 32$ & $0,24 \pm 0,02$ & $36068 \pm 2705$ & $2,28 \pm 0,06$ \\
\hline $\mathrm{CCSH}$ & $<1,4$ & $181 \pm 22$ & $61,5 \pm 0,3$ & $37115 \pm 2414$ & $2,2 \pm 0,2$ & $0,97 \pm 0,04$ & $1,8 \pm 0,1$ & $0,14 \pm 0,03$ & $<0,09$ & $859 \pm 26$ & $0,29 \pm 0,02$ & $39246 \pm 2941$ & $1,76 \pm 0,04$ \\
\hline CCDA & $<1,4$ & $115 \pm 13$ & $8,01 \pm 0,05$ & $30946 \pm 2342$ & $4,9 \pm 0,6$ & $0,98 \pm 0,04$ & $2,4 \pm 0,2$ & $0,13 \pm 0,03$ & $<0,09$ & $916 \pm 32$ & $0,2 \pm 0,03$ & $34030 \pm 6724$ & $2,19 \pm 0,07$ \\
\hline $\mathrm{CCDH}$ & $1,7 \pm 0,1$ & $120 \pm 14$ & $63,7 \pm 0,4$ & $37034 \pm 2646$ & $2,6 \pm 0,4$ & $0,77 \pm 0,04$ & $1,6 \pm 0,2$ & $0,13 \pm 0,02$ & $<0,09$ & $625 \pm 23$ & $0,17 \pm 0,03$ & $28849 \pm 5701$ & $1,68 \pm 0,05$ \\
\hline CCDV & $2,9 \pm 0,2$ & $98 \pm 12$ & $72 \pm 1$ & $28864 \pm 2264$ & $3,2 \pm 0,5$ & $0,85 \pm 0,04$ & $1,3 \pm 0,2$ & $0,17 \pm 0,03$ & $<0,09$ & $813 \pm 29$ & $0,16 \pm 0,02$ & $31772 \pm 6295$ & $2,26 \pm 0,07$ \\
\hline CCFA & $1,4 \pm 0,1$ & $111 \pm 13$ & $74 \pm 1$ & $32471 \pm 2455$ & $4,4 \pm 0,6$ & $0,88 \pm 0,04$ & $4,4 \pm 0,3$ & $0,18 \pm 0,02$ & $<0,09$ & $892 \pm 31$ & $0,21 \pm 0,03$ & $30489 \pm 6037$ & $2,36 \pm 0,07$ \\
\hline CCFV & $<1,4$ & $156 \pm 18$ & $70,5 \pm 0,5$ & $36874 \pm 2647$ & $5,7 \pm 0,7$ & $0,86 \pm 0,04$ & $2,9 \pm 0,2$ & $0,18 \pm 0,03$ & $<0,09$ & $1074 \pm 36$ & $0,25 \pm 0,03$ & $31890 \pm 6299$ & $2,68 \pm 0,08$ \\
\hline $\mathrm{CCHOV}$ & $1,6 \pm 0,2$ & $169 \pm 19$ & $59,0 \pm 0,4$ & $31737 \pm 2410$ & $4,5 \pm 0,6$ & $0,77 \pm 0,04$ & $1,2 \pm 0,2$ & $0,17 \pm 0,03$ & $<0,09$ & $991 \pm 34$ & $0,27 \pm 0,04$ & $29388 \pm 5822$ & $1,84 \pm 0,06$ \\
\hline $\mathrm{CCM}$ & $<1,4$ & $11 \pm 3$ & $87 \pm 1$ & $30636 \pm 2396$ & $3,2 \pm 0,5$ & $0,96 \pm 0,04$ & $1,6 \pm 0,2$ & $0,15 \pm 0,02$ & $<0,09$ & $950 \pm 33$ & $0,23 \pm 0,03$ & $33180 \pm 6555$ & $2,43 \pm 0,07$ \\
\hline CCEPA & $<1,4$ & $133 \pm 17$ & $67,2 \pm 0,4$ & $23732 \pm 2150$ & $<0,79$ & $0,64 \pm 0,03$ & $1,1 \pm 0,1$ & $<0,04$ & $<0,09$ & $435 \pm 20$ & $0,16 \pm 0,02$ & $33650 \pm 1401$ & $<0,53$ \\
\hline CCMS & $<1,4$ & $123 \pm 16$ & $15,2 \pm 0,2$ & $22585 \pm 2122$ & $<0,79$ & $0,79 \pm 0,04$ & $1,4 \pm 0,2$ & $0,16 \pm 0,03$ & $<0,09$ & $698 \pm 28$ & $<0,09$ & $27069 \pm 1096$ & $<0,53$ \\
\hline CCPLA & $<1,4$ & $<39$ & $50,1 \pm 0,3$ & $24108 \pm 2271$ & $<0,79$ & $1,06 \pm 0,05$ & $<0,82$ & $0,23 \pm 0,04$ & $<0,09$ & $795 \pm 30$ & $0,11 \pm 0,02$ & $37802 \pm 1572$ & $1,6 \pm 0,04$ \\
\hline CCMFP & $<1,4$ & $122 \pm 6$ & $50,2 \pm 0,2$ & $28172 \pm 1338$ & $1,9 \pm 0,1$ & $0,89 \pm 0,03$ & $2,1 \pm 0,1$ & $0,12 \pm 0,02$ & $<0,09$ & $744 \pm 16$ & $0,26 \pm 0,02$ & $24074 \pm 2542$ & $1,54 \pm 0,04$ \\
\hline CCMGA & $<1,4$ & $141 \pm 10$ & $38,1 \pm 0,2$ & $23883 \pm 1673$ & $<0,79$ & $0,98 \pm 0,04$ & $<0,82$ & $0,25 \pm 0,03$ & $<0,09$ & $1015 \pm 24$ & $0,32 \pm 0,03$ & $39445 \pm 2562$ & $2,32 \pm 0,06$ \\
\hline CCSPS & $<1,4$ & $89 \pm 7$ & $6,99 \pm 0,03$ & $23968 \pm 1695$ & $<0,79$ & $0,79 \pm 0,03$ & $2,2 \pm 0,2$ & $0,53 \pm 0,05$ & $<0,09$ & $676 \pm 18$ & $0,15 \pm 0,02$ & $45276 \pm 2922$ & $1,86 \pm 0,05$ \\
\hline
\end{tabular}




\begin{tabular}{|c|c|c|c|c|c|c|c|c|c|c|c|c|c|}
\hline & Lu & $\mathrm{Na}$ & $\mathbf{R b}$ & $\mathrm{Sb}$ & Sc & Se & Sm & $\mathrm{Ta}$ & $\mathrm{Tb}$ & Th & $\mathbf{U}$ & $\mathrm{Yb}$ & $\mathrm{Zn}$ \\
\hline CCDLA & $<0,05$ & $792 \pm 22$ & $26 \pm 2$ & $<0,21$ & $0,2 \pm 0,01$ & $<0,29$ & $0,114 \pm 0,005$ & $<0,22$ & $<0,05$ & $<0,38$ & $<0,48$ & $<0,30$ & $44 \pm 2$ \\
\hline CCDLV & $<0,05$ & $714 \pm 20$ & $27 \pm 1$ & $<0,21$ & $0,179 \pm 0,005$ & $<0,29$ & $0,089 \pm 0,004$ & $<0,22$ & $<0,05$ & $<0,38$ & $<0,48$ & $<0,30$ & $41 \pm 2$ \\
\hline CCLMA & $<0,05$ & $644 \pm 18$ & $29 \pm 2$ & $<0,21$ & $0,23 \pm 0,01$ & $0,5 \pm 0,3$ & $0,116 \pm 0,005$ & $<0,22$ & $<0,05$ & $<0,38$ & $<0,48$ & $<0,30$ & $45 \pm 2$ \\
\hline CCMG & $<0,05$ & $648 \pm 18$ & $24 \pm 1$ & $<0,21$ & $0,23 \pm 0,01$ & $<0,29$ & $0,13 \pm 0,01$ & $<0,22$ & $<0,05$ & $<0,38$ & $<0,48$ & $<0,30$ & $44 \pm 2$ \\
\hline сCMV & $<0,05$ & $665 \pm 19$ & $28 \pm 2$ & $<0,21$ & $0,24 \pm 0,01$ & $<0,29$ & $0,14 \pm 0,01$ & $<0,22$ & $<0,05$ & $<0,38$ & $<0,48$ & $<0,30$ & $44 \pm 2$ \\
\hline CCPZ & $<0,05$ & $940 \pm 26$ & $36 \pm 2$ & $<0,21$ & $0,34 \pm 0,01$ & $0,3 \pm 0,2$ & $0,17 \pm 0,01$ & $<0,22$ & $<0,05$ & $<0,38$ & $<0,48$ & $<0,30$ & $38 \pm 2$ \\
\hline $\mathrm{CCSH}$ & $<0,05$ & $667 \pm 19$ & $30 \pm 2$ & $<0,21$ & $0,23 \pm 0,01$ & $0,5 \pm 0,3$ & $0,13 \pm 0,01$ & $<0,22$ & $<0,05$ & $<0,38$ & $<0,48$ & $<0,30$ & $44 \pm 2$ \\
\hline CCDA & $<0,05$ & $475 \pm 38$ & $31 \pm 2$ & $<0,21$ & $0,25 \pm 0,01$ & $<0,29$ & $0,16 \pm 0,01$ & $<0,22$ & $<0,05$ & $<0,38$ & $<0,48$ & $<0,30$ & $36 \pm 2$ \\
\hline CCDH & $<0,05$ & $482 \pm 38$ & $25 \pm 2$ & $<0,21$ & $0,18 \pm 0,01$ & $<0,29$ & $<0,04$ & $<0,22$ & $<0,05$ & $<0,38$ & $<0,48$ & $<0,30$ & $33 \pm 2$ \\
\hline CCDV & $<0,05$ & $477 \pm 38$ & $24 \pm 2$ & $0,32 \pm 0,04$ & $0,23 \pm 0,01$ & $<0,29$ & $0,18 \pm 0,01$ & $<0,22$ & $<0,05$ & $<0,38$ & $<0,48$ & $<0,30$ & $29 \pm 2$ \\
\hline CCFA & $<0,05$ & $544 \pm 43$ & $26 \pm 2$ & $<0,21$ & $0,25 \pm 0,01$ & $<0,29$ & $0,19 \pm 0,01$ & $<0,22$ & $0,10 \pm 0,03$ & $<0,38$ & $<0,48$ & $<0,30$ & $38 \pm 2$ \\
\hline CCFV & $<0,05$ & $516 \pm 41$ & $25 \pm 2$ & $<0,21$ & $0,27 \pm 0,01$ & $<0,29$ & $0,20 \pm 0,01$ & $<0,22$ & $<0,05$ & $<0,38$ & $<0,48$ & $<0,30$ & $39 \pm 2$ \\
\hline CCHOV & $<0,05$ & $1015 \pm 81$ & $27 \pm 2$ & $<0,21$ & $0,27 \pm 0,01$ & $<0,29$ & $0,15 \pm 0,01$ & $<0,22$ & $<0,05$ & $<0,38$ & $<0,48$ & $<0,30$ & $41 \pm 2$ \\
\hline $\mathrm{CCM}$ & $<0,05$ & $507 \pm 40$ & $25 \pm 2$ & $<0,21$ & $0,25 \pm 0,01$ & $<0,29$ & $0,23 \pm 0,02$ & $<0,22$ & $<0,05$ & $<0,38$ & $<0,48$ & $<0,30$ & $38 \pm 2$ \\
\hline CCEPA & $<0,05$ & $957 \pm 29$ & $23 \pm 2$ & $<0,21$ & $0,136 \pm 0,004$ & $<0,29$ & $<0,04$ & $<0,22$ & $<0,05$ & $<0,38$ & $<0,48$ & $<0,30$ & $35 \pm 2$ \\
\hline CCMS & $<0,05$ & $430 \pm 13$ & $22 \pm 1$ & $<0,21$ & $0,21 \pm 0,01$ & $<0,29$ & $0,079 \pm 0,004$ & $<0,22$ & $<0,05$ & $<0,38$ & $<0,48$ & $<0,30$ & $40 \pm 2$ \\
\hline CCPLA & $<0,05$ & $564 \pm 18$ & $28 \pm 2$ & $<0,21$ & $0,26 \pm 0,01$ & $1,4 \pm 0,6$ & $0,14 \pm 0,01$ & $<0,22$ & $<0,05$ & $<0,38$ & $<0,48$ & $<0,30$ & $37 \pm 2$ \\
\hline CCMFP & $<0,05$ & $494 \pm 21$ & $31 \pm 1$ & $<0,21$ & $0,23 \pm 0,01$ & $<0,29$ & $0,151 \pm 0,005$ & $<0,22$ & $0,30 \pm 0,08$ & $<0,38$ & $<0,48$ & $<0,30$ & $26 \pm 1$ \\
\hline CCMGA & $<0,05$ & $987 \pm 28$ & $27 \pm 2$ & $<0,21$ & $0,35 \pm 0,01$ & $<0,29$ & $0,17 \pm 0,01$ & $<0,22$ & $<0,05$ & $<0,38$ & $<0,48$ & $<0,30$ & $37 \pm 2$ \\
\hline CCSPS & $<0,05$ & $1243 \pm 35$ & $43 \pm 2$ & $<0,21$ & $0,21 \pm 0,01$ & $<0,29$ & $0,113 \pm 0,004$ & $<0,22$ & $<0,05$ & $<0,38$ & $<0,48$ & $<0,30$ & $36 \pm 2$ \\
\hline
\end{tabular}


APENDICE A.2 - Concentração dos elementos em mg kg-1 nos cigarros aromatizados determinados por Analise por Ativação com Nêutrons Instrumental.

\begin{tabular}{|c|c|c|c|c|c|c|c|c|c|c|c|c|c|c|}
\hline & As & $\mathrm{Ba}$ & $\mathrm{Br}$ & $\mathrm{Ca}$ & $\mathrm{Ce}$ & Co & $\mathrm{Cr}$ & Cs & Eu & $\mathrm{Fe}$ & $\mathrm{Hf}$ & \multicolumn{2}{|c|}{$\mathrm{K}(\%)$} & La \\
\hline CADBM & $<1,4$ & $87 \pm 11$ & $21,2 \pm 0,3$ & $22584 \pm 1865$ & $1,8 \pm 0,3$ & $0,52 \pm 0,03$ & $2,3 \pm 0,2$ & $0,16 \pm 0,04$ & $<0,09$ & $504 \pm 25$ & $0,15 \pm 0,02$ & \multicolumn{2}{|c|}{$<3020$} & $1,4 \pm 0,1$ \\
\hline CAFF & $<1,4$ & $82 \pm 11$ & $39,4 \pm 0,5$ & $21978 \pm 1837$ & $2,1 \pm 0,3$ & $0,75 \pm 0,04$ & $1,9 \pm 0,2$ & $0,32 \pm 0,05$ & $<0,09$ & $896 \pm 38$ & $0,16 \pm 0,02$ & \multicolumn{2}{|c|}{$<3020$} & $3,8 \pm 0,2$ \\
\hline CAHM & $2,9 \pm 0,4$ & $113 \pm 14$ & $70 \pm 1$ & $20430 \pm 11739$ & $2 \pm 0,3$ & $0,72 \pm 0,04$ & $1,6 \pm 0,2$ & $0,4 \pm 0,1$ & $<0,09$ & $1031 \pm 42$ & $0,29 \pm 0,03$ & \multicolumn{2}{|c|}{$<3020$} & $2,0 \pm 0,1$ \\
\hline CALAM & $<1,4$ & $103 \pm 13$ & $42 \pm 1$ & $21414 \pm 11796$ & $0,9 \pm 0,2$ & $0,53 \pm 0,03$ & $2,6 \pm 0,2$ & $0,28 \pm 0,04$ & $<0,09$ & $512 \pm 26$ & $0,09 \pm 0,02$ & \multicolumn{2}{|c|}{$<3020$} & $1,4 \pm 0,1$ \\
\hline CALMM & $<1,4$ & $165 \pm 19$ & $50 \pm 1$ & $26092 \pm 2094$ & $2,2 \pm 0,3$ & $0,9 \pm 0,1$ & $1,7 \pm 0,2$ & $0,15 \pm 0,04$ & $<0,09$ & $769 \pm 34$ & $0,24 \pm 0,03$ & \multicolumn{2}{|c|}{$<3020$} & $3,0 \pm 0,2$ \\
\hline CALSF & $<1,4$ & $109 \pm 14$ & $72 \pm 1$ & $17948 \pm 11588$ & $2 \pm 0,3$ & $0,82 \pm 0,05$ & $2,2 \pm 0,2$ & $0,20 \pm 0,04$ & $<0,09$ & $737 \pm 33$ & $0,12 \pm 0,02$ & \multicolumn{2}{|c|}{$<3020$} & $2,4 \pm 0,1$ \\
\hline CAMFM & $2,1 \pm 0,3$ & $162 \pm 19$ & $44 \pm 1$ & $22137 \pm 11841$ & $2,3 \pm 0,3$ & $0,89 \pm 0,05$ & $1,4 \pm 0,2$ & $0,18 \pm 0,04$ & $<0,09$ & $662 \pm 30$ & $0,36 \pm 0,04$ & \multicolumn{2}{|c|}{$<3020$} & $3,1 \pm 0,2$ \\
\hline \multirow[t]{2}{*}{ CAMBI } & $<1,4$ & $143 \pm 23$ & $51,7 \pm 0,2$ & $40925 \pm 3746$ & $1,3 \pm 0,2$ & $1,07 \pm 0,05$ & $2,6 \pm 0,3$ & $0,13 \pm 0,03$ & $<0,09$ & $883 \pm 37$ & $0,27 \pm 0,02$ & \multicolumn{2}{|c|}{$33012 \pm 4515$} & $1,61 \pm 0,04$ \\
\hline & Lu & $\mathrm{Na}$ & $\mathrm{Rb}$ & $\mathrm{Sb}$ & Sc & $\mathrm{Se}$ & \multicolumn{2}{|c|}{$\mathrm{Sm}$} & $\mathrm{Ta}$ & $\mathrm{Tb}$ & Th & \multicolumn{2}{|l|}{$U$} & $\mathrm{Zn}$ \\
\hline CADBM & $<0,05$ & $1377 \pm 67$ & $21 \pm 2$ & $<0,21$ & $0,16 \pm 0,01$ & $<0,29$ & \multicolumn{2}{|c|}{$0,077 \pm 0,001$} & $<0,22$ & $<0,05$ & $<0,38$ & \multicolumn{2}{|l|}{$<0,48$} & $32 \pm 2$ \\
\hline CAFF & $<0,05$ & $677 \pm 35$ & $30 \pm 3$ & $<0,21$ & $0,29 \pm 0,01$ & $<0,29$ & \multicolumn{2}{|c|}{$0,17 \pm 0,01$} & $<0,22$ & $<0,05$ & $<0,38$ & \multicolumn{2}{|l|}{$<0,48$} & $43 \pm 3$ \\
\hline CAHM & $<0,05$ & $701 \pm 701$ & $28 \pm 3$ & $<0,21$ & $0,28 \pm 0,01$ & $<0,29$ & \multicolumn{2}{|c|}{$0,11 \pm 0,01$} & $<0,22$ & $<0,05$ & $<0,38$ & \multicolumn{2}{|l|}{$<0,48$} & $31 \pm 2$ \\
\hline CALAM & $<0,05$ & $1377 \pm 67$ & $29 \pm 3$ & $<0,21$ & $0,17 \pm 0,01$ & $<0,29$ & \multicolumn{2}{|c|}{$0,077 \pm 0,001$} & $<0,22$ & $<0,05$ & $<0,38$ & \multicolumn{2}{|l|}{$<0,48$} & $39 \pm 2$ \\
\hline CALMM & $<0,05$ & $897 \pm 46$ & $33 \pm 3$ & $<0,21$ & $0,25 \pm 0,01$ & $<0,29$ & 0,14 & 0,01 & $<0,22$ & $<0,05$ & $<0,38$ & $<0,48$ & $<0,30$ & $36 \pm 3$ \\
\hline CALSF & $<0,05$ & $608 \pm 34$ & $26 \pm 3$ & $<0,21$ & $0,25 \pm 0,01$ & $<0,29$ & $0,1 \pm$ & 0,01 & $<0,22$ & $<0,05$ & $<0,38$ & $<0,48$ & $<0,30$ & $41 \pm 3$ \\
\hline CAMFM & $<0,05$ & $877 \pm 44$ & $28 \pm 3$ & $<0,21$ & $0,22 \pm 0,01$ & $<0,29$ & 0,09 & 0,01 & $<0,22$ & $<0,05$ & $<0,38$ & $<0,48$ & $<0,30$ & $38 \pm 2$ \\
\hline CAMBI & $<0,05$ & $516 \pm 30$ & $25 \pm 1$ & $<0,21$ & $0,24 \pm 0,01$ & $<0,29$ & 0,111 & 0,004 & $<0,22$ & $<0,05$ & $<0,38$ & $<0,48$ & $<0,30$ & $42 \pm 2$ \\
\hline
\end{tabular}


APENDICE A.3 - Concentração dos elementos em mg kg-1 nos cigarros de palha determinados por Analise por Ativação com Nêutrons Instrumental.

\begin{tabular}{|c|c|c|c|c|c|c|c|c|c|c|c|c|c|}
\hline & As & $\mathrm{Ba}$ & $\mathrm{Br}$ & $\mathrm{Ca}$ & $\mathrm{Ce}$ & Co & $\mathrm{Cr}$ & Cs & $\mathrm{Eu}$ & $\mathrm{Fe}$ & $\mathrm{Hf}$ & K & La \\
\hline CPC & $1,9 \pm 0,2$ & $161 \pm 13$ & $47,1 \pm 0,3$ & $19970 \pm 1928$ & $2,9 \pm 0,3$ & $0,47 \pm 0,03$ & $<0,82$ & $<0,04$ & $<0,09$ & $567 \pm 37$ & $0,35 \pm 0,03$ & $16238 \pm 506$ & $11,3 \pm 0,3$ \\
\hline CPD & $<1,4$ & $76 \pm 9$ & $44,1 \pm 0,3$ & $34050 \pm 2915$ & $3,4 \pm 0,3$ & $0,45 \pm 0,03$ & $1,3 \pm 0,2$ & $0,4 \pm 0,1$ & $<0,09$ & $1009 \pm 61$ & $0,25 \pm 0,03$ & $30507 \pm 939$ & $7,2 \pm 0,2$ \\
\hline CPP & $<1,4$ & $92 \pm 10$ & $52,4 \pm 0,3$ & $22143 \pm 2098$ & $6 \pm 1$ & $1,3 \pm 0,1$ & $3,3 \pm 0,3$ & $<0,04$ & $0,13 \pm 0,03$ & $1755 \pm 100$ & $0,17 \pm 0,03$ & $15312 \pm 480$ & $31 \pm 1$ \\
\hline CPPF & $3,9 \pm 0,2$ & $103 \pm 17$ & $29,7 \pm 0,2$ & $66161 \pm 5661$ & $5,5 \pm 0,5$ & $0,61 \pm 0,03$ & $1,3 \pm 0,2$ & $0,34 \pm 0,04$ & $<0,09$ & $999 \pm 41$ & $0,26 \pm 0,02$ & $27767 \pm 3805$ & $8,4 \pm 0,2$ \\
\hline CPRN & $3,2 \pm 0,2$ & $111 \pm 18$ & $61,6 \pm 0,2$ & $37304 \pm 3438$ & $2,7 \pm 0,3$ & $0,44 \pm 0,02$ & $1,6 \pm 0,3$ & $0,29 \pm 0,04$ & $<0,09$ & $815 \pm 35$ & $0,27 \pm 0,03$ & $34863 \pm 4768$ & $2,2 \pm 0,1$ \\
\hline СРСРС & $<1,4$ & $88 \pm 6$ & $29,8 \pm 0,2$ & $22855 \pm 1599$ & $1,7 \pm 0,2$ & $0,62 \pm 0,03$ & $0,8 \pm 0,1$ & $0,13 \pm 0,02$ & $<0,09$ & $461 \pm 13$ & $0,23 \pm 0,02$ & $53170 \pm 3431$ & $1,3 \pm 0,04$ \\
\hline CPFN & $<1,4$ & $93 \pm 7$ & $46,4 \pm 0,3$ & $<7147$ & $4,9 \pm 0,4$ & $<0,13$ & $0,6 \pm 0,1$ & $0,25 \pm 0,03$ & $<0,09$ & $452 \pm 14$ & $0,14 \pm 0,02$ & $33095 \pm 2141$ & $6,6 \pm 0,2$ \\
\hline CPTV & $<1,4$ & $76 \pm 6$ & $153 \pm 1$ & $37532 \pm 2529$ & $5,3 \pm 0,4$ & $1,8 \pm 0,1$ & $0,7 \pm 0,1$ & $0,20 \pm 0,03$ & $0,2 \pm 0,1$ & $389 \pm 13$ & $0,17 \pm 0,02$ & $46231 \pm 2988$ & $14,8 \pm 0,4$ \\
\hline CPCPE & $<1,4$ & $144 \pm 11$ & $52,4 \pm 0,4$ & $27637 \pm 2398$ & $6 \pm 1$ & $0,56 \pm 0,03$ & $1,5 \pm 0,2$ & $0,18 \pm 0,04$ & $<0,09$ & $862 \pm 30$ & $0,21 \pm 0,03$ & $16222 \pm 2881$ & $14 \pm 1$ \\
\hline CPCR & $1,5 \pm 0,2$ & $67 \pm 9$ & $67,8 \pm 0,5$ & $39365 \pm 3140$ & $7 \pm 1$ & $0,55 \pm 0,03$ & $6 \pm 1$ & $0,3 \pm 0,1$ & $<0,09$ & $3021 \pm 85$ & $0,39 \pm 0,04$ & $8232 \pm 1542$ & $10 \pm 1$ \\
\hline CPPG & $1,8 \pm 0,2$ & $197 \pm 15$ & $38,2 \pm 0,4$ & $41826 \pm 3311$ & $7 \pm 1$ & $1,2 \pm 0,1$ & $3,5 \pm 0,4$ & $0,3 \pm 0,1$ & $0,1 \pm 0,05$ & $1782 \pm 55$ & $0,52 \pm 0,04$ & $9390 \pm 1750$ & $10 \pm 1$ \\
\hline CPSP & $<1,4$ & $111 \pm 10$ & $57,7 \pm 0,5$ & $37720 \pm 2998$ & $5 \pm 1$ & $0,88 \pm 0,04$ & $2,9 \pm 0,4$ & $0,23 \pm 0,05$ & $<0,09$ & $1284 \pm 41$ & $0,24 \pm 0,03$ & $8006 \pm 1655$ & $8 \pm 1$ \\
\hline CPVC & $6,4 \pm 0,4$ & $140 \pm 12$ & $37,7 \pm 0,4$ & $45259 \pm 3462$ & $11 \pm 1$ & $1,3 \pm 0,1$ & $8 \pm 1$ & $0,5 \pm 0,1$ & $0,15 \pm 0,03$ & $3264 \pm 91$ & $1,2 \pm 0,1$ & $8347 \pm 1591$ & $15 \pm 1$ \\
\hline
\end{tabular}




\begin{tabular}{|c|c|c|c|c|c|c|c|c|c|c|c|c|c|}
\hline & Lu & $\mathrm{Na}$ & $\mathbf{R b}$ & $\mathrm{Sb}$ & Sc & Se & Sm & $\mathrm{Ta}$ & $\mathrm{Tb}$ & Th & $\mathbf{U}$ & $\mathrm{Yb}$ & $\mathrm{Zn}$ \\
\hline CPC & $<0,05$ & $325 \pm 8$ & $25 \pm 2$ & $<0,21$ & $0,149 \pm 0,005$ & $<0,29$ & $0,24 \pm 0,01$ & $<0,22$ & $<0,05$ & $<0,38$ & $<0,48$ & $<0,30$ & $30 \pm 1$ \\
\hline CPD & $<0,05$ & $940 \pm 22$ & $15 \pm 1$ & $<0,21$ & $0,33 \pm 0,01$ & $<0,29$ & $0,121 \pm 0,004$ & $<0,22$ & $<0,05$ & $<0,38$ & $<0,48$ & $<0,30$ & $57 \pm 2$ \\
\hline CPP & $<0,05$ & $283 \pm 7$ & $23 \pm 2$ & $<0,21$ & $0,53 \pm 0,02$ & $<0,29$ & $0,48 \pm 0,01$ & $<0,22$ & $<0,05$ & $0,38 \pm 0,03$ & $<0,48$ & $<0,30$ & $47 \pm 2$ \\
\hline CPPF & $<0,05$ & $590 \pm 35$ & $26 \pm 2$ & $<0,21$ & $0,37 \pm 0,01$ & $0,5 \pm 0,4$ & $0,49 \pm 0,02$ & $<0,22$ & $<0,05$ & $<0,38$ & $<0,48$ & $<0,30$ & $69 \pm 3$ \\
\hline CPRN & $<0,05$ & $453 \pm 27$ & $51 \pm 3$ & $<0,21$ & $0,22 \pm 0,01$ & $<0,29$ & $0,115 \pm 0,004$ & $<0,22$ & $<0,05$ & $<0,38$ & $<0,48$ & $<0,30$ & $60 \pm 3$ \\
\hline СРСРС & $<0,05$ & $2577 \pm 73$ & $20 \pm 1$ & $<0,21$ & $0,143 \pm 0,004$ & $<0,29$ & $0,84 \pm 0,003$ & $<0,22$ & $<0,05$ & $<0,38$ & $<0,48$ & $<0,30$ & $50 \pm 3$ \\
\hline CPFN & $<0,05$ & $172 \pm 5$ & $<4,5$ & $<0,21$ & $0,3 \pm 0,01$ & $<0,29$ & $0,2 \pm 0,01$ & $<0,22$ & $<0,05$ & $<0,38$ & $<0,48$ & $<0,30$ & $137 \pm 7$ \\
\hline CPTV & $<0,05$ & $476 \pm 14$ & $46 \pm 3$ & $<0,21$ & $0,12 \pm 0,004$ & $<0,29$ & $0,58 \pm 0,02$ & $<0,22$ & $<0,05$ & $<0,38$ & $<0,48$ & $<0,30$ & $70 \pm 4$ \\
\hline СРCPE & $<0,05$ & $304 \pm 22$ & $51 \pm 4$ & $<0,21$ & $0,22 \pm 0,01$ & $<0,29$ & $0,34 \pm 0,01$ & $<0,22$ & $<0,05$ & $<0,38$ & $<0,48$ & $<0,30$ & $70 \pm 4$ \\
\hline CPCR & $<0,05$ & $190 \pm 14$ & $19 \pm 2$ & $<0,21$ & $0,69 \pm 0,02$ & $<0,29$ & $0,45 \pm 0,02$ & $<0,22$ & $0,06 \pm 0,02$ & $0,82 \pm 0,05$ & $<0,48$ & $<0,30$ & $43 \pm 2$ \\
\hline CPPG & $<0,05$ & $139 \pm 11$ & $18 \pm 2$ & $<0,21$ & $0,69 \pm 0,02$ & $<0,29$ & $0,5 \pm 0,02$ & $<0,22$ & $<0,05$ & $0,75 \pm 0,05$ & $<0,48$ & $<0,30$ & $58 \pm 3$ \\
\hline CPSP & $<0,05$ & $222 \pm 18$ & $14 \pm 1$ & $<0,21$ & $0,44 \pm 0,01$ & $<0,29$ & $0,36 \pm 0,01$ & $<0,22$ & $<0,05$ & $0,5 \pm 0,04$ & $<0,48$ & $<0,30$ & $73 \pm 4$ \\
\hline CPVC & $<0,05$ & $527 \pm 37$ & $21 \pm 2$ & $<0,21$ & $1,29 \pm 0,04$ & $<0,29$ & $0,76 \pm 0,03$ & $<0,22$ & $0,14 \pm 0,05$ & $1,6 \pm 0,1$ & $<0,48$ & $0,39 \pm 0,04$ & $68 \pm 3$ \\
\hline
\end{tabular}


APENDICE A.4 - Concentração dos elementos em mg kg-1 nos charutos determinados por Analise por Ativação com Nêutrons Instrumental.

\begin{tabular}{|c|c|c|c|c|c|c|c|c|c|c|c|c|c|}
\hline & As & $\mathrm{Ba}$ & $\mathrm{Br}$ & $\mathrm{Ca}$ & $\mathrm{Ce}$ & Co & $\mathrm{Cr}$ & Cs & $\mathrm{Eu}$ & $\mathrm{Fe}$ & $\mathrm{Hf}$ & K & La \\
\hline $\mathrm{CHDF}$ & $<1,4$ & $78 \pm 11$ & $137 \pm 1$ & $30367 \pm 2578$ & $1,9 \pm 0,2$ & $0,49 \pm 0,03$ & $4,7 \pm 0,4$ & $0,2 \pm 0,04$ & $<0,09$ & $497 \pm 22$ & $0,41 \pm 0,04$ & $43813 \pm 1846$ & $<0,53$ \\
\hline CHDNE & $<1,4$ & $92 \pm 12$ & $61,5 \pm 0,4$ & $33533 \pm 2775$ & $<0,79$ & $0,29 \pm 0,02$ & $1,1 \pm 0,2$ & $0,09 \pm 0,03$ & $<0,09$ & $488 \pm 22$ & $0,27 \pm 0,03$ & $55898 \pm 2284$ & $0,74 \pm 0,02$ \\
\hline CHMPC & $2,4 \pm 0,2$ & $37 \pm 7$ & $134 \pm 1$ & $44692 \pm 3478$ & $<0,79$ & $0,37 \pm 0,02$ & $1,8 \pm 0,2$ & $0,15 \pm 0,03$ & $<0,09$ & $618 \pm 26$ & $0,35 \pm 0,03$ & $40489 \pm 1733$ & $0,88 \pm 0,03$ \\
\hline $\mathrm{CHNI}$ & $2,5 \pm 0,2$ & $165 \pm 21$ & $48,8 \pm 0,4$ & $33779 \pm 2789$ & $1,8 \pm 0,2$ & $0,58 \pm 0,03$ & $3,5 \pm 0,3$ & $0,11 \pm 0,03$ & $<0,09$ & $500 \pm 22$ & $0,52 \pm 0,04$ & $40962 \pm 1753$ & $0,89 \pm 0,03$ \\
\hline CHDNC & $<1,4$ & $104 \pm 5$ & $118,9 \pm 0,5$ & $26221 \pm 1274$ & $<0,79$ & $0,45 \pm 0,02$ & $1,6 \pm 0,1$ & $0,15 \pm 0,02$ & $<0,09$ & $604 \pm 14$ & $0,423 \pm 0,03$ & $26446 \pm 2799$ & $0,68 \pm 0,02$ \\
\hline \multirow[t]{2}{*}{ CHMPE } & $<1,4$ & $74 \pm 4$ & $164 \pm 1$ & $46237 \pm 1971$ & $<0,79$ & $0,39 \pm 0,02$ & $1,3 \pm 0,1$ & $0,14 \pm 0,01$ & $<0,09$ & $492 \pm 12$ & $0,32 \pm 0,02$ & $20098 \pm 2114$ & $0,73 \pm 0,02$ \\
\hline & Lu & $\mathrm{Na}$ & $\mathrm{Rb}$ & $\mathrm{Sb}$ & Sc & Se & $\mathrm{Sm}$ & $\mathrm{Ta}$ & $\mathrm{Tb}$ & Th & U & $\mathrm{Yb}$ & $\mathrm{Zn}$ \\
\hline CHDF & $<0,05$ & $724 \pm 23$ & $28 \pm 2$ & $<0,21$ & $0,115 \pm 0,004$ & $<0,29$ & $0,030 \pm 0,003$ & $<0,22$ & $<0,05$ & $<0,38$ & $<0,48$ & $<0,30$ & $75 \pm 3$ \\
\hline CHDNE & $<0,05$ & $517 \pm 16$ & $29 \pm 2$ & $<0,21$ & $0,73 \pm 0,003$ & $<0,29$ & $0,029 \pm 0,003$ & $<0,22$ & $<0,05$ & $<0,38$ & $<0,48$ & $<0,30$ & $80 \pm 3$ \\
\hline CHMPC & $<0,05$ & $940 \pm 29$ & $24 \pm 2$ & $<0,21$ & $0,114 \pm 0,004$ & $<0,29$ & $0,034 \pm 0,003$ & $<0,22$ & $<0,05$ & $<0,38$ & $<0,48$ & $<0,30$ & $79 \pm 3$ \\
\hline CHNI & $<0,05$ & $951 \pm 30$ & $43 \pm 3$ & $<0,21$ & $0,131 \pm 0,004$ & $<0,29$ & $0,034 \pm 0,003$ & $<0,22$ & $<0,05$ & $<0,38$ & $<0,48$ & $<0,30$ & $69 \pm 3$ \\
\hline CHDNC & $<0,05$ & $358 \pm 16$ & $26 \pm 1$ & $<0,21$ & $0,097 \pm 0,002$ & $0,3 \pm 0,1$ & $0,023 \pm 0,002$ & $<0,22$ & $<0,05$ & $<0,38$ & $<0,48$ & $<0,30$ & $36 \pm 1$ \\
\hline CHMPE & $<0,05$ & $760 \pm 33$ & $19 \pm 1$ & $<0,21$ & $0,085 \pm 0,002$ & $<0,29$ & $0,014 \pm 0,001$ & $<0,22$ & $<0,05$ & $<0,38$ & $<0,48$ & $<0,30$ & $69 \pm 2$ \\
\hline
\end{tabular}


APENDICE A.5 - Concentração dos elementos em $\mathrm{mg} \mathrm{kg}^{-1}$ nos fumos de corda e rapés determinados por Analise por Ativação com Nêutrons Instrumental.

\begin{tabular}{|c|c|c|c|c|c|c|c|c|c|c|c|c|c|}
\hline & As & $\mathrm{Ba}$ & $\mathrm{Br}$ & $\mathrm{Ca}$ & $\mathrm{Ce}$ & Co & $\mathrm{Cr}$ & Cs & $\mathrm{Eu}$ & $\mathrm{Fe}$ & $\mathrm{Hf}$ & $\mathbf{K}$ & La \\
\hline $\begin{array}{c}\text { FCAA } \\
\text { L }\end{array}$ & $\begin{array}{c}1,5 \pm \\
0,2\end{array}$ & $52 \pm 8$ & $\begin{array}{c}52,6 \pm \\
0,3\end{array}$ & $\begin{array}{c}23427 \pm \\
2139\end{array}$ & $2,4 \pm 0,3$ & $0,26 \pm 0,02$ & $1,2 \pm 0,3$ & $0,20 \pm 0,04$ & $<0,09$ & $449 \pm 31$ & $\begin{array}{c}0,24 \pm \\
0,03\end{array}$ & $46125 \pm 1427$ & $7,2 \pm 0,2$ \\
\hline $\begin{array}{c}\text { FCCP } \\
\text { R }\end{array}$ & $<1,4$ & $41 \pm 7$ & $\begin{array}{c}48,1 \pm \\
0,3\end{array}$ & $\begin{array}{c}15860 \pm \\
1717\end{array}$ & $2,1 \pm 0,3$ & $0,11 \pm 0,02$ & $<0,82$ & $0,14 \pm 0,03$ & $<0,09$ & $320 \pm 25$ & $\begin{array}{c}0,28 \pm \\
0,03\end{array}$ & $45430 \pm 1409$ & $8,8 \pm 0,3$ \\
\hline FCDJ & $<1,4$ & $59 \pm 8$ & $99 \pm 1$ & $\begin{array}{c}21153 \pm \\
1996\end{array}$ & $4,9 \pm 0,5$ & $0,76 \pm 0,05$ & $1,3 \pm 0,2$ & $<0,04$ & $<0,09$ & $448 \pm 32$ & $\begin{array}{c}0,19 \pm \\
0,02\end{array}$ & $35325 \pm 1108$ & $32 \pm 1$ \\
\hline FCP & $<1,4$ & $\begin{array}{c}123 \pm \\
13\end{array}$ & $125 \pm 1$ & $\begin{array}{c}29263 \pm \\
2639\end{array}$ & $7 \pm 1$ & $0,71 \pm 0,05$ & $3,5 \pm 0,4$ & $0,23 \pm 0,05$ & $0,16 \pm 0,03$ & $\begin{array}{c}1673 \pm \\
96\end{array}$ & $0,2 \pm 0,03$ & $29168 \pm 932$ & $72 \pm 2$ \\
\hline FCPF & $<1,4$ & $\begin{array}{c}112 \pm \\
11\end{array}$ & $\begin{array}{c}37,6 \pm \\
0,3\end{array}$ & $\begin{array}{c}25330 \pm \\
2352\end{array}$ & $25 \pm 2$ & $1,2 \pm 0,1$ & $2,6 \pm 0,3$ & $<0,04$ & $0,15 \pm 0,03$ & $\begin{array}{c}1536 \pm \\
86\end{array}$ & $\begin{array}{c}0,19 \pm \\
0,02\end{array}$ & $43057 \pm 1347$ & $101 \pm 3$ \\
\hline FCA1 & $\begin{array}{c}2,2 \pm \\
0,1\end{array}$ & $\begin{array}{c}157 \pm \\
15\end{array}$ & $\begin{array}{c}35,2 \pm \\
0,3\end{array}$ & $\begin{array}{c}21348 \pm \\
1492\end{array}$ & $2,2 \pm 0,2$ & $0,88 \pm 0,04$ & $1,7 \pm 0,4$ & $0,11 \pm 0,03$ & $<0,09$ & $779 \pm 33$ & $\begin{array}{c}0,15 \pm \\
0,02\end{array}$ & $43241 \pm 3279$ & $\begin{array}{c}1,39 \pm \\
0,04\end{array}$ \\
\hline FCA2 & $\begin{array}{c}2,1 \pm \\
0,1\end{array}$ & $\begin{array}{c}167 \pm \\
16\end{array}$ & $\begin{array}{c}59,2 \pm \\
0,3\end{array}$ & $\begin{array}{c}25394 \pm \\
1719\end{array}$ & $4,6 \pm 0,4$ & $1,3 \pm 0,1$ & $3 \pm 1$ & $0,31 \pm 0,04$ & $<0,09$ & $137 \pm 43$ & $\begin{array}{c}0,25 \pm \\
0,02\end{array}$ & $48207 \pm 3650$ & $4,1 \pm 0,1$ \\
\hline FCA3 & $<1,4$ & $\begin{array}{c}155 \pm \\
15\end{array}$ & $\begin{array}{c}41,7 \pm \\
0,3\end{array}$ & $\begin{array}{c}22527 \pm \\
1545\end{array}$ & $2,3 \pm 0,2$ & $1,00 \pm 0,04$ & $1,9 \pm 0,4$ & $0,25 \pm 0,03$ & $<0,09$ & $736 \pm 32$ & $\begin{array}{c}0,25 \pm \\
0,02\end{array}$ & $48163 \pm 3643$ & $\begin{array}{l}1,81 \pm \\
0,05\end{array}$ \\
\hline FCA4 & $\begin{array}{c}2,1 \pm \\
0,2\end{array}$ & $\begin{array}{c}133 \pm \\
14\end{array}$ & $126 \pm 1$ & $\begin{array}{c}27952 \pm \\
1884\end{array}$ & $14 \pm 1$ & $1,3 \pm 0,1$ & $5 \pm 1$ & $0,29 \pm 0,04$ & $0,14 \pm 0,03$ & $\begin{array}{c}1176 \pm \\
49\end{array}$ & $0,6 \pm 0,1$ & $48330 \pm 3659$ & $\begin{array}{c}10,6 \pm \\
0,3\end{array}$ \\
\hline FCA5 & $\begin{array}{c}3,2 \pm \\
0,2\end{array}$ & $\begin{array}{c}136 \pm \\
14\end{array}$ & $114 \pm 1$ & $\begin{array}{c}25270 \pm \\
1759\end{array}$ & $12 \pm 1$ & $1,4 \pm 0,1$ & $4 \pm 1$ & $0,23 \pm 0,04$ & $0,12 \pm 0,02$ & $655 \pm 30$ & $\begin{array}{c}0,33 \pm \\
0,03\end{array}$ & $54347 \pm 4108$ & $\begin{array}{c}11,2 \pm \\
0,3\end{array}$ \\
\hline FCA6 & $\begin{array}{c}1,6 \pm \\
0,1\end{array}$ & $\begin{array}{c}176 \pm \\
17\end{array}$ & $\begin{array}{c}35,4 \pm \\
0,2\end{array}$ & $\begin{array}{c}24759 \pm \\
1659\end{array}$ & $2,3 \pm 0,3$ & $1,02 \pm 0,04$ & $2,2 \pm 0,5$ & $0,13 \pm 0,03$ & $<0,09$ & $988 \pm 41$ & $\begin{array}{c}0,26 \pm \\
0,03\end{array}$ & $52663 \pm 3973$ & $\begin{array}{c}1,61 \pm \\
0,04\end{array}$ \\
\hline FCA7 & $\begin{array}{c}1,9 \pm \\
0,1\end{array}$ & $\begin{array}{c}153 \pm \\
15\end{array}$ & $\begin{array}{c}56,9 \pm \\
0,3\end{array}$ & $\begin{array}{c}24904 \pm \\
1696\end{array}$ & $2,9 \pm 0,3$ & $1,2 \pm 0,1$ & $2 \pm 1$ & $0,13 \pm 0,03$ & $<0,09$ & $975 \pm 41$ & $\begin{array}{c}0,24 \pm \\
0,02\end{array}$ & $50272 \pm 3792$ & $2,9 \pm 0,1$ \\
\hline FCA8 & $\begin{array}{c}1,6 \pm \\
0,1\end{array}$ & $\begin{array}{c}152 \pm \\
10\end{array}$ & $\begin{array}{c}33,5 \pm \\
0,2\end{array}$ & $\begin{array}{c}19268 \pm \\
1077\end{array}$ & $2,3 \pm 0,2$ & $1,05 \pm 0,04$ & $2,1 \pm 0,2$ & $0,22 \pm 0,03$ & $<0,09$ & $897 \pm 31$ & $\begin{array}{c}0,31 \pm \\
0,04\end{array}$ & $41804 \pm 4965$ & $\begin{array}{c}1,68 \pm \\
0,04\end{array}$ \\
\hline FCA9 & $<1,4$ & $\begin{array}{c}143 \pm \\
10\end{array}$ & $\begin{array}{c}34,3 \pm \\
0,2\end{array}$ & $\begin{array}{c}21037 \pm \\
1133\end{array}$ & $2,7 \pm 0,2$ & $0,83 \pm 0,03$ & $1,1 \pm 0,1$ & $0,14 \pm 0,03$ & $<0,09$ & $804 \pm 28$ & $\begin{array}{c}0,27 \pm \\
0,04\end{array}$ & $45043 \pm 5319$ & $\begin{array}{c}1,87 \pm \\
0,05\end{array}$ \\
\hline $\begin{array}{c}\text { FCA1 } \\
0\end{array}$ & $\begin{array}{c}2,0 \pm \\
0,1\end{array}$ & $\begin{array}{c}170 \pm \\
11\end{array}$ & $\begin{array}{c}38,1 \pm \\
0,2\end{array}$ & $\begin{array}{c}24228 \pm \\
1270\end{array}$ & $1,9 \pm 0,2$ & $0,9 \pm 0,04$ & $1,5 \pm 0,1$ & $0,12 \pm 0,03$ & $<0,09$ & $783 \pm 27$ & $\begin{array}{c}0,27 \pm \\
0,04\end{array}$ & $43275 \pm 5112$ & $\begin{array}{l}1,79 \pm \\
0,05\end{array}$ \\
\hline FCC & $<1,4$ & $82 \pm 6$ & $\begin{array}{c}21,7 \pm \\
0,2\end{array}$ & $\begin{array}{c}20332 \pm \\
1111\end{array}$ & $13 \pm 1$ & $0,20 \pm 0,01$ & $1,8 \pm 0,1$ & $0,12 \pm 0,03$ & $0,10 \pm 0,02$ & $826 \pm 29$ & $\begin{array}{c}0,14 \pm \\
0,02\end{array}$ & $15458 \pm 1889$ & $\begin{array}{c}11,1 \pm \\
0,3\end{array}$ \\
\hline FCG & $<1,4$ & $135 \pm 9$ & $\begin{array}{c}33,6 \pm \\
0,2\end{array}$ & $\begin{array}{c}32080 \pm \\
1642\end{array}$ & $3,1 \pm 0,3$ & $0,52 \pm 0,03$ & $1,8 \pm 0,2$ & $0,45 \pm 0,04$ & $0,11 \pm 0,02$ & $820 \pm 29$ & $\begin{array}{c}0,15 \pm \\
0,02\end{array}$ & $26118 \pm 3094$ & $\begin{array}{c}10,9 \pm \\
0,3\end{array}$ \\
\hline $\mathrm{FCl}$ & $<1,4$ & $59 \pm 6$ & $226 \pm 1$ & $\begin{array}{c}21631 \pm \\
1171\end{array}$ & $1,5 \pm 0,2$ & $0,63 \pm 0,03$ & $2,1 \pm 0,2$ & $0,27 \pm 0,04$ & $<0,09$ & $\begin{array}{c}1292 \pm \\
43\end{array}$ & $<0,09$ & $23622 \pm 2846$ & $\begin{array}{c}0,79 \pm \\
0,02\end{array}$ \\
\hline FCSC & $<1,4$ & $17 \pm 3$ & $\begin{array}{c}68,9 \pm \\
0,4\end{array}$ & $\begin{array}{c}14397 \pm \\
847\end{array}$ & $1,9 \pm 0,2$ & $0,15 \pm 0,01$ & $1,4 \pm 0,1$ & $0,11 \pm 0,02$ & $<0,09$ & $951 \pm 32$ & $<0,09$ & $26572 \pm 3170$ & $\begin{array}{c}1,22 \pm \\
0,03\end{array}$ \\
\hline
\end{tabular}




\begin{tabular}{|c|c|c|c|c|c|c|c|c|c|c|c|c|c|c|}
\hline & As & $\mathrm{Ba}$ & $\mathrm{Br}$ & $\mathrm{Ca}$ & $\mathrm{Ce}$ & Co & & $\mathrm{Cr}$ & Cs & $\mathrm{Eu}$ & $\mathrm{Fe}$ & $\mathrm{Hf}$ & $\mathrm{K}$ & La \\
\hline FCDD & $<1,4$ & $77 \pm 13$ & $\begin{array}{c}87,3 \pm \\
0,3\end{array}$ & $\begin{array}{c}41877 \pm \\
3872\end{array}$ & $5,5 \pm 0,5$ & $0,52 \pm 0,03$ & & $1,5 \pm 0,2$ & $0,22 \pm 0,03$ & $0,09 \pm 0,02$ & $634 \pm 29$ & $\begin{array}{c}0,24 \pm \\
0,02\end{array}$ & $29343 \pm 4018$ & $7,6 \pm 0,2$ \\
\hline $\begin{array}{c}\text { FCD } \\
\text { M }\end{array}$ & $<1,4$ & $\begin{array}{c}128 \pm \\
21\end{array}$ & $\begin{array}{c}93,2 \pm \\
0,4\end{array}$ & $\begin{array}{c}45909 \pm \\
4140\end{array}$ & $3,5 \pm 0,3$ & $\begin{array}{c}1,05 \pm \\
0,05\end{array}$ & $\begin{array}{c}1,9 \pm \\
0,3\end{array}$ & $\begin{array}{c}0,23 \pm \\
0,04\end{array}$ & $<0,09$ & $943 \pm 39$ & $\begin{array}{c}0,42 \pm \\
0,03\end{array}$ & $\begin{array}{c}31545 \pm \\
4318\end{array}$ & \multicolumn{2}{|c|}{$5,6 \pm 0,1$} \\
\hline $\begin{array}{c}\text { FCAC } \\
\text { E }\end{array}$ & $<1,4$ & $71 \pm 4$ & $43 \pm 0,2$ & $\begin{array}{c}14268 \pm \\
824\end{array}$ & $2,1 \pm 0,1$ & $\begin{array}{c}0,32 \pm \\
0,01\end{array}$ & $<0,82$ & $\begin{array}{c}0,09 \pm \\
0,01\end{array}$ & $<0,09$ & $306 \pm 8$ & $\begin{array}{c}0,1 \pm \\
0,01\end{array}$ & $\begin{array}{c}13390 \pm \\
1409\end{array}$ & \multicolumn{2}{|c|}{$1,28 \pm 0,03$} \\
\hline & As & $\mathrm{Ba}$ & $\mathrm{Br}$ & $\mathrm{Ca}$ & $\mathrm{Ce}$ & Co & $\mathrm{Cr}$ & Cs & $\mathrm{Eu}$ & $\mathrm{Fe}$ & $\mathrm{Hf}$ & $\mathrm{K}$ & \multicolumn{2}{|l|}{ La } \\
\hline$\underset{\mathrm{H}}{\mathrm{FCDB}}$ & $<1,4$ & $147 \pm 7$ & $\begin{array}{c}38,8 \pm \\
0,2\end{array}$ & $\begin{array}{c}24546 \pm \\
1205\end{array}$ & $2,6 \pm 0,2$ & $\begin{array}{c}0,52 \pm \\
0,02\end{array}$ & $\begin{array}{c}1,0 \pm \\
0,1\end{array}$ & $\begin{array}{c}0,16 \pm \\
0,02\end{array}$ & $<0,09$ & $593 \pm 14$ & $\begin{array}{c}0,31 \pm \\
0,02\end{array}$ & $\begin{array}{c}19766 \pm \\
1953\end{array}$ & \multicolumn{2}{|c|}{$1,97 \pm 0,05$} \\
\hline $\begin{array}{l}\text { FCD } \\
\text { MP }\end{array}$ & $<1,4$ & $\begin{array}{c}207 \pm \\
14\end{array}$ & $\begin{array}{c}46,1 \pm \\
0,3\end{array}$ & $\begin{array}{c}39705 \pm \\
2664\end{array}$ & $14 \pm 1$ & $\begin{array}{c}0,79 \pm \\
0,03\end{array}$ & $\begin{array}{c}1,8 \pm \\
0,2\end{array}$ & $\begin{array}{c}0,19 \pm \\
0,03\end{array}$ & $0,11 \pm 0,02$ & $1915 \pm 41$ & $\begin{array}{c}0,30 \pm \\
0,03\end{array}$ & $\begin{array}{c}30736 \pm \\
1986\end{array}$ & \multicolumn{2}{|c|}{$16,8 \pm 0,4$} \\
\hline FCDR & $<1,4$ & $88 \pm 7$ & $\begin{array}{c}118,9 \pm \\
0,5\end{array}$ & $\begin{array}{c}32922 \pm \\
2231\end{array}$ & $8 \pm 1$ & $\begin{array}{c}1,05 \pm \\
0,04\end{array}$ & $<0,82$ & $\begin{array}{c}0,19 \pm \\
0,03\end{array}$ & $<0,09$ & $457 \pm 14$ & $\begin{array}{c}0,18 \pm \\
0,02\end{array}$ & $\begin{array}{c}42687 \pm \\
2753\end{array}$ & \multicolumn{2}{|c|}{$14,2 \pm 0,4$} \\
\hline $\begin{array}{c}\text { FCAC } \\
\text { C }\end{array}$ & $<1,4$ & $72 \pm 7$ & $\begin{array}{c}42,1 \pm \\
0,4\end{array}$ & $\begin{array}{c}13964 \pm \\
1531\end{array}$ & $1,4 \pm 0,2$ & $\begin{array}{c}0,27 \pm \\
0,02\end{array}$ & $<0,82$ & $<0,04$ & $<0,09$ & $360 \pm 17$ & $\begin{array}{c}0,11 \pm \\
0,02\end{array}$ & $\begin{array}{c}11440 \pm \\
2062\end{array}$ & \multicolumn{2}{|c|}{$2,2 \pm 0,1$} \\
\hline $\begin{array}{l}\text { FCA } \\
\text { MO }\end{array}$ & $<1,4$ & $75 \pm 7$ & $\begin{array}{c}37,3 \pm \\
0,4\end{array}$ & $\begin{array}{c}11463 \pm \\
1390\end{array}$ & $1,2 \pm 0,2$ & $\begin{array}{c}0,25 \pm \\
0,02\end{array}$ & $<0,82$ & $<0,04$ & $<0,09$ & $219 \pm 13$ & $\begin{array}{c}0,12 \pm \\
0,02\end{array}$ & $\begin{array}{c}11347 \pm \\
2077\end{array}$ & \multicolumn{2}{|c|}{$2,2 \pm 0,1$} \\
\hline RPAR & $<1,4$ & $94 \pm 16$ & $\begin{array}{c}61,1 \pm \\
0,3\end{array}$ & $\begin{array}{c}37349 \pm \\
3508\end{array}$ & $7 \pm 1$ & $\begin{array}{c}0,80 \pm \\
0,04\end{array}$ & $\begin{array}{c}3,9 \pm \\
0,5\end{array}$ & $\begin{array}{c}0,25 \pm \\
0,02\end{array}$ & $<0,09$ & $2946 \pm 109$ & $1,8 \pm 0,1$ & $\begin{array}{c}30009 \pm \\
4105\end{array}$ & \multicolumn{2}{|c|}{$8,6 \pm 0,2$} \\
\hline $\begin{array}{c}\text { RPC } \\
\text { M }\end{array}$ & $<1,4$ & $\begin{array}{c}139 \pm \\
23\end{array}$ & $\begin{array}{c}72,9 \pm \\
0,3\end{array}$ & $\begin{array}{c}44319 \pm \\
4094\end{array}$ & $8 \pm 1$ & $\begin{array}{c}1,30 \pm \\
0,06\end{array}$ & $7 \pm 1$ & $0,5 \pm 0,1$ & $<0,09$ & $4070 \pm 148$ & $1,9 \pm 0,1$ & $\begin{array}{c}36456 \pm \\
4991\end{array}$ & \multicolumn{2}{|c|}{$8,7 \pm 0,2$} \\
\hline
\end{tabular}

\begin{tabular}{|c|c|c|c|c|c|c|c|c|c|c|c|c|c|}
\hline & Lu & $\mathrm{Na}$ & $\mathrm{Rb}$ & $\mathrm{Sb}$ & Sc & $\mathrm{Se}$ & $\mathrm{Sm}$ & $\mathrm{Ta}$ & $\mathrm{Tb}$ & Th & $U$ & $\mathrm{Yb}$ & $\mathrm{Zn}$ \\
\hline $\begin{array}{c}\text { FCAA } \\
\text { L }\end{array}$ & $<0,05$ & $\begin{array}{l}604 \pm \\
15\end{array}$ & $12 \pm 1$ & $<0,21$ & $\begin{array}{c}0,075 \pm \\
0,003\end{array}$ & $<0,29$ & $0,042 \pm 0,002$ & $\begin{array}{c}< \\
0,22\end{array}$ & $<0,05$ & $<0,38$ & $<0,48$ & $\begin{array}{c}< \\
0,30\end{array}$ & $33 \pm 2$ \\
\hline $\begin{array}{c}\text { FCCP } \\
\mathbf{R}\end{array}$ & $<0,05$ & $\begin{array}{c}479 \pm \\
12\end{array}$ & $13 \pm 1$ & $<0,21$ & $\begin{array}{c}0,108 \pm \\
0,004\end{array}$ & $<0,29$ & $0,065 \pm 0,003$ & $\begin{array}{c}< \\
0,22\end{array}$ & $<0,05$ & $<0,38$ & $<0,48$ & $\begin{array}{c}< \\
0,30\end{array}$ & $49 \pm 2$ \\
\hline FCDJ & $<0,05$ & $\begin{array}{c}457 \pm \\
11\end{array}$ & $23 \pm 2$ & $<0,21$ & $\begin{array}{c}0,129 \pm \\
0,004\end{array}$ & $<0,29$ & $0,29 \pm 0,01$ & $\begin{array}{l}< \\
0,22\end{array}$ & $<0,05$ & $<0,38$ & $<0,48$ & $\begin{array}{l}< \\
0,30\end{array}$ & $73 \pm 3$ \\
\hline FCP & $<0,05$ & $182 \pm 5$ & $22 \pm 2$ & $<0,21$ & $0,46 \pm 0,01$ & $0,4 \pm 0,1$ & $0,67 \pm 0,02$ & $\begin{array}{l}< \\
0,22\end{array}$ & $<0,05$ & $\begin{array}{c}0,48 \pm \\
0,04\end{array}$ & $<0,48$ & $\begin{array}{l}< \\
0,30\end{array}$ & $37 \pm 2$ \\
\hline FCPF & $<0,05$ & $119 \pm 3$ & $25 \pm 2$ & $<0,21$ & $0,33 \pm 0,01$ & $<0,29$ & $0,57 \pm 0,02$ & $\begin{array}{l}< \\
0,22\end{array}$ & $<0,05$ & $\begin{array}{c}0,55 \pm \\
0,04\end{array}$ & $<0,48$ & 0,30 & $66 \pm 3$ \\
\hline FCA1 & $<0,05$ & $\begin{array}{c}973 \pm \\
25\end{array}$ & $22 \pm 1$ & $<0,21$ & $\begin{array}{c}0,163 \pm \\
0,005\end{array}$ & $<0,29$ & $0,118 \pm 0,003$ & $\begin{array}{l}< \\
0,22\end{array}$ & $<0,05$ & $<0,38$ & $<0,48$ & $\begin{array}{l}< \\
0,30\end{array}$ & $108 \pm 3$ \\
\hline FCA2 & $<0,05$ & $\begin{array}{c}884 \pm \\
23\end{array}$ & $32 \pm 2$ & $<0,21$ & $0,27 \pm 0,01$ & $<0,29$ & $0,27 \pm 0,01$ & $\begin{array}{l}< \\
0,22\end{array}$ & $<0,05$ & $<0,38$ & $<0,48$ & < & $104 \pm 3$ \\
\hline FCA3 & $<0,05$ & $\begin{array}{c}881 \pm \\
23\end{array}$ & $29 \pm 2$ & $<0,21$ & $\begin{array}{c}0,189 \pm \\
0,005\end{array}$ & $<0,29$ & $0,136 \pm 0,003$ & $\begin{array}{c}< \\
0,22\end{array}$ & $<0,05$ & $<0,38$ & $<0,48$ & $\begin{array}{c}< \\
0,30\end{array}$ & $106 \pm 3$ \\
\hline
\end{tabular}




\begin{tabular}{|c|c|c|c|c|c|c|c|c|c|c|c|c|c|}
\hline & Lu & $\mathrm{Na}$ & $\mathrm{Rb}$ & $\mathrm{Sb}$ & Sc & $\mathrm{Se}$ & $\mathrm{Sm}$ & $\mathrm{Ta}$ & $\mathrm{Tb}$ & Th & $U$ & $\mathrm{Yb}$ & $\mathrm{Zn}$ \\
\hline FCA4 & $<0,05$ & $\begin{array}{c}1214 \pm \\
32\end{array}$ & $24 \pm 1$ & $<0,21$ & $0,61 \pm 0,02$ & $0,7 \pm 0,4$ & $0,8 \pm 0,02$ & $\begin{array}{c}<< \\
0,22\end{array}$ & $\begin{array}{c}0,05 \pm \\
0,02\end{array}$ & $0,6 \pm 0,1$ & $<0,48$ & $\begin{array}{c}0,32 \pm \\
0,04\end{array}$ & $116 \pm 4$ \\
\hline FCA5 & $<0,05$ & $\begin{array}{c}1236 \pm \\
32\end{array}$ & $27 \pm 2$ & $<0,21$ & $0,36 \pm 0,01$ & $0,4 \pm 0,2$ & $0,8 \pm 0,02$ & $\begin{array}{c}< \\
0,22\end{array}$ & $\begin{array}{c}0,12 \pm \\
0,03\end{array}$ & $0,4 \pm 0,1$ & $<0,48$ & $<0,30$ & $108 \pm 3$ \\
\hline FCA6 & $<0,05$ & $\begin{array}{c}942 \pm \\
24\end{array}$ & $27 \pm 2$ & $<0,21$ & $0,24 \pm 0,01$ & $0,3 \pm 0,2$ & $0,132 \pm 0,004$ & $\begin{array}{c}< \\
0,22\end{array}$ & $<0,05$ & $<0,38$ & $<0,48$ & $<0,30$ & $93 \pm 3$ \\
\hline FCA7 & $<0,05$ & $\begin{array}{c}683 \pm \\
18\end{array}$ & $30 \pm 2$ & $<0,21$ & $0,27 \pm 0,01$ & $<0,29$ & $0,177 \pm 0,005$ & $\begin{array}{c}< \\
0,22\end{array}$ & $<0,05$ & $<0,38$ & $<0,48$ & $<0,30$ & $106 \pm 3$ \\
\hline FCA8 & $<0,05$ & $\begin{array}{c}735 \pm \\
37\end{array}$ & $27 \pm 2$ & $<0,21$ & $0,24 \pm 0,01$ & $<0,29$ & $<0,04$ & $\begin{array}{c}< \\
0,22\end{array}$ & $<0,05$ & $<0,38$ & $<0,48$ & $<0,30$ & $100 \pm 3$ \\
\hline FCA9 & $<0,05$ & $\begin{array}{l}710 \pm \\
35\end{array}$ & $27 \pm 2$ & $<0,21$ & $0,21 \pm 0,01$ & $<0,29$ & $<0,04$ & $\begin{array}{c}< \\
0,22\end{array}$ & $<0,05$ & $<0,38$ & $<0,48$ & $<0,30$ & $100 \pm 3$ \\
\hline $\begin{array}{c}\text { FCA1 } \\
0\end{array}$ & $<0,05$ & $\begin{array}{c}799 \pm \\
40\end{array}$ & $26 \pm 2$ & $<0,21$ & $0,22 \pm 0,01$ & $<0,29$ & $0,126 \pm 0,004$ & $\begin{array}{c}< \\
0,22\end{array}$ & $<0,05$ & $<0,38$ & $<0,48$ & $<0,30$ & $107 \pm 3$ \\
\hline FCC & $<0,05$ & $73 \pm 4$ & $14 \pm 1$ & $<0,21$ & $0,24 \pm 0,01$ & $<0,29$ & $0,6 \pm 0,02$ & $\begin{array}{c}< \\
0,22\end{array}$ & $<0,05$ & $<0,38$ & $<0,48$ & $<0,30$ & $37 \pm 1$ \\
\hline FCG & $<0,05$ & $75 \pm 4$ & $44 \pm 3$ & $<0,21$ & $0,23 \pm 0,01$ & $<0,29$ & $0,52 \pm 0,02$ & $\begin{array}{l}< \\
0,22\end{array}$ & $<0,05$ & $<0,38$ & $<0,48$ & $<0,30$ & $127 \pm 4$ \\
\hline $\mathrm{FCl}$ & $<0,05$ & $156 \pm 8$ & $25 \pm 2$ & $<0,21$ & $0,36 \pm 0,01$ & $<0,29$ & $0,040 \pm 0,002$ & $\begin{array}{c}< \\
0,22\end{array}$ & $<0,05$ & $<0,38$ & $<0,48$ & $<0,30$ & $53 \pm 2$ \\
\hline FCSC & $<0,05$ & $119 \pm 6$ & $16 \pm 1$ & $<0,21$ & $\begin{array}{c}0,168 \pm \\
0,005\end{array}$ & $<0,29$ & $0,048 \pm 0,002$ & $\begin{array}{c}< \\
0,22\end{array}$ & $<0,05$ & $<0,38$ & $<0,48$ & $<0,30$ & $75 \pm 2$ \\
\hline FCDD & $<0,05$ & $\begin{array}{c}516 \pm \\
30\end{array}$ & $25 \pm 1$ & $<0,21$ & $0,18 \pm 0,01$ & $<0,29$ & $0,34 \pm 0,01$ & $\begin{array}{c}< \\
0,22\end{array}$ & $\begin{array}{c}0,08 \pm \\
0,02\end{array}$ & $0,4 \pm 0,1$ & $<0,48$ & $<0,30$ & $74 \pm 3$ \\
\hline FCDM & $<0,05$ & $\begin{array}{c}422 \pm \\
25\end{array}$ & $28 \pm 2$ & $<0,21$ & $0,3 \pm 0,01$ & $0,6 \pm 0,5$ & $0,26 \pm 0,01$ & $\begin{array}{c}< \\
0,22\end{array}$ & $<0,05$ & $0,4 \pm 0,1$ & $<0,48$ & $<0,30$ & $71 \pm 3$ \\
\hline FCACE & $<0,05$ & $\begin{array}{c}532 \pm \\
23\end{array}$ & $17 \pm 1$ & $<0,21$ & $\begin{array}{l}0,52 \pm \\
0,001\end{array}$ & $<0,29$ & $0,111 \pm 0,004$ & $\begin{array}{c}< \\
0,22\end{array}$ & $<0,05$ & $<0,38$ & $<0,48$ & $<0,30$ & $17 \pm 0,5$ \\
\hline FCDBH & $<0,05$ & $\begin{array}{c}829 \pm \\
36\end{array}$ & $23 \pm 1$ & $<0,21$ & $\begin{array}{l}0,183 \pm \\
0,004\end{array}$ & $<0,29$ & $0,127 \pm 0,004$ & $\begin{array}{c}< \\
0,22\end{array}$ & $<0,05$ & $<0,38$ & $<0,48$ & $<0,30$ & $20 \pm 1$ \\
\hline FCDMP & $<0,05$ & $162 \pm 5$ & $46 \pm 3$ & $<0,21$ & $0,45 \pm 0,01$ & $<0,29$ & $0,68 \pm 0,02$ & $\begin{array}{c}< \\
0,22\end{array}$ & $<0,05$ & $0,9 \pm 0,1$ & $<0,48$ & $<0,30$ & $82 \pm 4$ \\
\hline FCDR & $<0,05$ & $\begin{array}{c}523 \pm \\
15\end{array}$ & $37 \pm 2$ & $<0,21$ & $0,19 \pm 0,01$ & $<0,29$ & $0,42 \pm 0,01$ & $\begin{array}{c}< \\
0,22\end{array}$ & $<0,05$ & $<0,38$ & $<0,48$ & $<0,30$ & $73 \pm 4$ \\
\hline FCACC & $<0,05$ & $\begin{array}{c}590 \pm \\
41\end{array}$ & $20 \pm 2$ & $<0,21$ & $\begin{array}{c}0,061 \pm \\
0,002\end{array}$ & $<0,29$ & $0,49 \pm 0,004$ & $\begin{array}{c}< \\
0,22\end{array}$ & $<0,05$ & $<0,38$ & $<0,48$ & $<0,30$ & $22 \pm 1$ \\
\hline FCAMO & $<0,05$ & $\begin{array}{c}495 \pm \\
35\end{array}$ & $22 \pm 2$ & $<0,21$ & $\begin{array}{l}0,05 \pm \\
0,002\end{array}$ & $<0,29$ & $0,043 \pm 0,004$ & $\begin{array}{c}< \\
0,22\end{array}$ & $<0,05$ & $<0,38$ & $<0,48$ & $<0,30$ & $33 \pm 2$ \\
\hline RPAR & $<0,05$ & $\begin{array}{c}722 \pm \\
43\end{array}$ & $33 \pm 3$ & $<0,21$ & $0,58 \pm 0,02$ & $<0,29$ & $0,40 \pm 0,01$ & $\begin{array}{c}< \\
0,22\end{array}$ & $<0,05$ & $1,0 \pm 0,2$ & $<0,48$ & $<0,30$ & $84 \pm 4$ \\
\hline RPCM & $<0,05$ & $\begin{array}{c}638 \pm \\
38\end{array}$ & $40 \pm 2$ & $<0,21$ & $1,01 \pm 0,03$ & $1,8 \pm 1,2$ & $0,50 \pm 0,02$ & $\begin{array}{c}< \\
0,22\end{array}$ & $<0,05$ & $1,2 \pm 0,2$ & $<0,48$ & $<0,30$ & $75 \pm 3$ \\
\hline
\end{tabular}


APENDICE B.1 - Concentração dos elementos em $\mathrm{mg} \mathrm{kg}^{-1}$ (* em \%) nos cigarros não aromatizados determinados por Espectrometria de raios- $X$

\begin{tabular}{cccccccc}
\hline & ${ }^{*} \mathbf{A l}$ & As & Br & ${ }^{*} \mathbf{C a}$ & Cd & ${ }^{*} \mathbf{C l}$ & Co \\
\hline CCDA & $0,060 \pm 0,004$ & $0,065 \pm 0,001$ & $75 \pm 7$ & $1,71 \pm 0,03$ & $1,00 \pm 0,03$ & $1,02 \pm 0,01$ & $0,81 \pm 0,02$ \\
CCDH & $0,041 \pm 0,004$ & $0,062 \pm 0,002$ & $40 \pm 4$ & $1,67 \pm 0,01$ & $0,39 \pm 0,01$ & $0,744 \pm 0,004$ & $0,63 \pm 0,01$ \\
CCDV & $0,066 \pm 0,005$ & $0,062 \pm 0,002$ & $69 \pm 6$ & $1,72 \pm 0,03$ & $0,31 \pm 0,01$ & $1,08 \pm 0,01$ & $0,51 \pm 0,04$ \\
CCEPA & $0,050 \pm 0,004$ & $0,062 \pm 0,001$ & $65 \pm 5$ & $1,76 \pm 0,02$ & $0,54 \pm 0,01$ & $0,902 \pm 0,003$ & $0,45 \pm 0,04$ \\
CCHOV & $0,078 \pm 0,003$ & $0,062 \pm 0,001$ & $63 \pm 5$ & $1,82 \pm 0,02$ & $0,83 \pm 0,01$ & $0,848 \pm 0,004$ & $0,68 \pm 0,04$ \\
CCM & $0,090 \pm 0,003$ & $0,065 \pm 0,001$ & $59 \pm 3$ & $1,60 \pm 0,01$ & $<0,21$ & $1,29 \pm 0,01$ & $0,95 \pm 0,03$ \\
CCMFP & $0,094 \pm 0,003$ & $0,061 \pm 0,002$ & $40 \pm 3$ & $1,66 \pm 0,02$ & $<0,21$ & $0,511 \pm 0,002$ & $0,73 \pm 0,04$ \\
CCMS & $0,068 \pm 0,004$ & $0,061 \pm 0,001$ & $42 \pm 2$ & $1,71 \pm 0,03$ & $1,05 \pm 0,01$ & $0,744 \pm 0,004$ & $0,79 \pm 0,01$ \\
CCPLA & $0,074 \pm 0,004$ & $0,061 \pm 0,001$ & $49 \pm 4$ & $1,71 \pm 0,01$ & $<0,21$ & $0,497 \pm 0,001$ & $0,59 \pm 0,01$ \\
CCPZ & $0,085 \pm 0,003$ & $0,063 \pm 0,002$ & $77 \pm 4$ & $1,75 \pm 0,01$ & $1,34 \pm 0,01$ & $0,920 \pm 0,002$ & $1,0 \pm 0,2$ \\
CCDLA & $0,042 \pm 0,005$ & $0,062 \pm 0,002$ & $47 \pm 1$ & $1,45 \pm 0,01$ & $0,31 \pm 0,03$ & $0,69 \pm 0,01$ & $0,75 \pm 0,04$ \\
CCDLV & $0,058 \pm 0,003$ & $0,064 \pm 0,001$ & $55 \pm 3$ & $1,55 \pm 0,01$ & $0,30 \pm 0,03$ & $0,955 \pm 0,004$ & $0,75 \pm 0,01$ \\
CCFA & $0,043 \pm 0,003$ & $0,062 \pm 0,002$ & $39 \pm 3$ & $1,64 \pm 0,01$ & $1,17 \pm 0,01$ & $0,762 \pm 0,004$ & $0,66 \pm 0,01$ \\
CCLMA & $0,080 \pm 0,001$ & $0,062 \pm 0,002$ & $<3,4$ & $1,66 \pm 0,02$ & $<0,21$ & $0,652 \pm 0,004$ & $0,78 \pm 0,03$ \\
CCMG & $0,069 \pm 0,001$ & $0,061 \pm 0,002$ & $52 \pm 5$ & $1,65 \pm 0,01$ & $<0,21$ & $0,512 \pm 0,003$ & $0,68 \pm 0,01$ \\
CCMV & $0,062 \pm 0,004$ & $0,062 \pm 0,001$ & $54 \pm 8$ & $1,64 \pm 0,01$ & $0,35 \pm 0,02$ & $0,488 \pm 0,004$ & $1,20 \pm 0,05$ \\
CCFV & $0,062 \pm 0,004$ & $0,062 \pm 0,002$ & $60 \pm 3$ & $1,82 \pm 0,03$ & $<0,21$ & $0,941 \pm 0,002$ & $0,82 \pm 0,04$ \\
CCSH & $0,063 \pm 0,004$ & $0,061 \pm 0,001$ & $54 \pm 4$ & $1,70 \pm 0,01$ & $<0,21$ & $0,667 \pm 0,004$ & $0,60 \pm 0,04$ \\
CCSPS & $0,057 \pm 0,004$ & $0,064 \pm 0,002$ & $50 \pm 7$ & $1,62 \pm 0,01$ & $1,6 \pm 0,2$ & $0,788 \pm 0,004$ & $0,67 \pm 0,01$
\end{tabular}




\begin{tabular}{ccccccccc}
\hline & $\mathbf{C r}$ & $\mathbf{C s}$ & $\mathbf{C u}$ & ${ }^{*} \mathrm{Fe}$ & $\mathbf{H g}$ & $\mathbf{I}$ & ${ }^{*} \mathbf{K}$ \\
\hline CCDA & $2,5 \pm 0,3$ & $0,047 \pm 0,001$ & $6,0 \pm 0,2$ & $0,062 \pm 0,002$ & $0,032 \pm 0,001$ & $205 \pm 6$ & $2,65 \pm 0,02$ & $5,8 \pm 0,4$ \\
CCDH & $1,06 \pm 0,05$ & $0,0442 \pm 0,004$ & $5,1 \pm 0,1$ & $0,050 \pm 0,003$ & $0,032 \pm 0,001$ & $215 \pm 7$ & $2,47 \pm 0,01$ & $7,9 \pm 0,4$ \\
CCDV & $0,95 \pm 0,05$ & $0,048 \pm 0,004$ & $4,9 \pm 0,1$ & $0,067 \pm 0,002$ & $0,029 \pm 0,001$ & $205 \pm 8$ & $3,01 \pm 0,01$ & $4,9 \pm 0,4$ \\
CCEPA & $0,97 \pm 0,05$ & $0,048 \pm 0,001$ & $5,1 \pm 0,2$ & $0,0405 \pm 0,0003$ & $0,031 \pm 0,001$ & $209,9 \pm 0,1$ & $2,84 \pm 0,01$ & $3,7 \pm 0,4$ \\
CCHOV & $1,04 \pm 0,04$ & $0,050 \pm 0,001$ & $5,0 \pm 0,3$ & $0,073 \pm 0,001$ & $0,029 \pm 0,001$ & $207 \pm 7$ & $2,51 \pm 0,01$ & $<1,4$ \\
CCM & $0,96 \pm 0,01$ & $0,041 \pm 0,004$ & $4,9 \pm 0,2$ & $0,050 \pm 0,003$ & $0,024 \pm 0,001$ & $202 \pm 6$ & $3,27 \pm 0,01$ & $7,2 \pm 0,4$ \\
CCMFP & $1,01 \pm 0,02$ & $0,048 \pm 0,001$ & $4,4 \pm 0,1$ & $0,065 \pm 0,002$ & $0,024 \pm 0,001$ & $199 \pm 4$ & $3,25 \pm 0,01$ & $3,7 \pm 0,4$ \\
CCMS & $1,07 \pm 0,05$ & $0,048 \pm 0,001$ & $4,9 \pm 0,1$ & $0,057 \pm 0,001$ & $0,030 \pm 0,001$ & $220 \pm 6$ & $2,46 \pm 0,02$ & $7,1 \pm 0,5$ \\
CCPLA & $1,00 \pm 0,03$ & $0,0468 \pm 0,004$ & $4,8 \pm 0,1$ & $0,064 \pm 0,001$ & $0,027 \pm 0,001$ & $218 \pm 12$ & $2,94 \pm 0,01$ & $2,4 \pm 0,4$ \\
CCPZ & $1,0 \pm 0,1$ & $0,047 \pm 0,001$ & $4,7 \pm 0,1$ & $0,086 \pm 0,003$ & $0,027 \pm 0,001$ & $207 \pm 2$ & $3,16 \pm 0,02$ & $1,9 \pm 0,4$ \\
CCDLA & $1,00 \pm 0,05$ & $0,045 \pm 0,002$ & $5,1 \pm 0,4$ & $0,045 \pm 0,001$ & $0,033 \pm 0,001$ & $217 \pm 8$ & $2,42 \pm 0,03$ & $12 \pm 1$ \\
CCDLV & $0,94 \pm 0,02$ & $0,041 \pm 0,001$ & $4,6 \pm 0,1$ & $0,040 \pm 0,003$ & $0,027 \pm 0,001$ & $201 \pm 7$ & $3,41 \pm 0,01$ & $5,8 \pm 0,2$ \\
CCFA & $1,08 \pm 0,02$ & $0,047 \pm 0,001$ & $5,3 \pm 0,1$ & $0,059 \pm 0,001$ & $0,034 \pm 0,001$ & $196 \pm 3$ & $2,02 \pm 0,01$ & $16 \pm 1$ \\
CCLMA & $0,99 \pm 0,02$ & $0,043 \pm 0,001$ & $4,81 \pm 0,04$ & $0,043 \pm 0,003$ & $0,025 \pm 0,001$ & $204 \pm 4$ & $3,47 \pm 0,02$ & $8,9 \pm 0,5$ \\
CCMG & $0,97 \pm 0,05$ & $0,044 \pm 0,001$ & $4,8 \pm 0,2$ & $0,065 \pm 0,001$ & $0,025 \pm 0,001$ & $204 \pm 5$ & $2,81 \pm 0,01$ & $3,7 \pm 0,1$ \\
CCMV & $1,02 \pm 0,05$ & $0,043 \pm 0,002$ & $4,9 \pm 0,2$ & $0,051 \pm 0,003$ & $0,029 \pm 0,001$ & $209 \pm 4$ & $2,63 \pm 0,02$ & $8 \pm 1$ \\
CCFV & $1,08 \pm 0,03$ & $0,045 \pm 0,001$ & $4,7 \pm 0,2$ & $0,077 \pm 0,001$ & $0,026 \pm 0,001$ & $192 \pm 4$ & $2,95 \pm 0,01$ & $2,05 \pm 0,4$ \\
CCSH & $0,95 \pm 0,04$ & $0,045 \pm 0,001$ & $4,4 \pm 0,1$ & $0,072 \pm 0,001$ & $0,024 \pm 0,001$ & $205 \pm 3$ & $3,54 \pm 0,01$ & $1,8 \pm 0,2$ \\
CCSPS & $0,81 \pm 0,05$ & $0,043 \pm 0,001$ & $5,0 \pm 0,1$ & $0,042 \pm 0,003$ & $0,032 \pm 0,001$ & $217 \pm 10$ & $2,77 \pm 0,01$ & $11 \pm 1$
\end{tabular}




\begin{tabular}{|c|c|c|c|c|c|c|c|c|}
\hline & ${ }^{*} \mathrm{Mg}$ & ${ }^{*} \mathrm{Mn}$ & Nd & $\mathrm{Ni}$ & ${ }^{*} \mathbf{P}$ & $\mathrm{Pb}$ & $\mathbf{R b}$ & *S \\
\hline CCDA & $1,09 \pm 0,04$ & $0,021 \pm 0,001$ & $18 \pm 1$ & $1,1 \pm 0,1$ & $0,16 \pm 0,01$ & $0,40 \pm 0,04$ & $20,0 \pm 0,5$ & $0,276 \pm 0,001$ \\
\hline $\mathrm{CCDH}$ & $0,94 \pm 0,04$ & $0,015 \pm 0,001$ & $5,5 \pm 0,4$ & $1,5 \pm 0,2$ & $0,14 \pm 0,01$ & $0,92 \pm 0,03$ & $19,4 \pm 0,1$ & $0,270 \pm 0,001$ \\
\hline CCDV & $0,94 \pm 0,03$ & $0,021 \pm 0,001$ & $<5,02$ & $1,32 \pm 0,02$ & $0,162 \pm 0,004$ & $1,0 \pm 0,1$ & $20,0 \pm 0,4$ & $0,290 \pm 0,001$ \\
\hline CCEPA & $1,10 \pm 0,04$ & $0,021 \pm 0,001$ & $<5,02$ & $1,3 \pm 0,1$ & $0,19 \pm 0,01$ & $1,36 \pm 0,03$ & $19,9 \pm 0,3$ & $0,300 \pm 0,001$ \\
\hline CCHOV & $0,84 \pm 0,04$ & $0,025 \pm 0,001$ & $6,4 \pm 0,2$ & $1,1 \pm 0,1$ & $0,16 \pm 0,01$ & $<0,32$ & $19,7 \pm 0,1$ & $0,262 \pm 0,001$ \\
\hline CCM & $1,22 \pm 0,04$ & $0,011 \pm 0,001$ & $<5,02$ & $1,18 \pm 0,04$ & $0,162 \pm 0,004$ & $1,41 \pm 0,03$ & $20,0 \pm 0,2$ & $0,304 \pm 0,002$ \\
\hline CCMFP & $0,82 \pm 0,03$ & $0,022 \pm 0,001$ & $<5,02$ & $1,4 \pm 0,2$ & $0,202 \pm 0,004$ & $1,2 \pm 0,1$ & $19,8 \pm 0,1$ & $0,307 \pm 0,001$ \\
\hline CCMS & $0,65 \pm 0,04$ & $0,019 \pm 0,001$ & $8,1 \pm 0,3$ & $1,00 \pm 0,02$ & $0,163 \pm 0,01$ & $0,81 \pm 0,03$ & $19,6 \pm 0,3$ & $0,275 \pm 0,002$ \\
\hline CCPLA & $0,82 \pm 0,03$ & $0,024 \pm 0,001$ & $<5,02$ & $0,72 \pm 0,02$ & $0,20 \pm 0,01$ & $0,53 \pm 0,03$ & $19,9 \pm 0,2$ & $0,301 \pm 0,001$ \\
\hline CCPZ & $0,76 \pm 0,01$ & $0,026 \pm 0,001$ & $<5,02$ & $1,2 \pm 0,1$ & $0,18 \pm 0,01$ & $1,07 \pm 0,03$ & $19,9 \pm 0,3$ & $0,291 \pm 0,002$ \\
\hline CCDLA & $0,60 \pm 0,04$ & $0,015 \pm 0,001$ & $9,3 \pm 0,5$ & $1,85 \pm 0,05$ & $0,14 \pm 0,01$ & $1,7 \pm 0,4$ & $20,0 \pm 0,2$ & $0,255 \pm 0,003$ \\
\hline CCDLV & $0,90 \pm 0,05$ & $0,011 \pm 0,001$ & $<5,02$ & $1,14 \pm 0,2$ & $0,21 \pm 0,01$ & $<0,32$ & $20,0 \pm 0,2$ & $0,320 \pm 0,002$ \\
\hline CCFA & $0,82 \pm 0,03$ & $0,014 \pm 0,001$ & $11,2 \pm 0,3$ & $2,2 \pm 0,2$ & $0,11 \pm 0,01$ & $<0,32$ & $19,4 \pm 0,2$ & $0,241 \pm 0,001$ \\
\hline CCLMA & $0,90 \pm 0,04$ & $0,015 \pm 0,001$ & $<5,02$ & $1,75 \pm 0,2$ & $0,21 \pm 0,01$ & $<0,32$ & $19,8 \pm 0,1$ & $0,334 \pm 0,003$ \\
\hline CCMG & $0,78 \pm 0,04$ & $0,021 \pm 0,001$ & $<5,02$ & $1,14 \pm 0,02$ & $0,18 \pm 0,01$ & $1,48 \pm 0,03$ & $19,9 \pm 0,4$ & $0,307 \pm 0,001$ \\
\hline CCMV & $0,53 \pm 0,02$ & $0,014 \pm 0,001$ & $7,3 \pm 0,3$ & $1,6 \pm 0,1$ & $0,16 \pm 0,01$ & $1,28 \pm 0,03$ & $19,8 \pm 0,1$ & $0,284 \pm 0,002$ \\
\hline CCFV & $1,1 \pm 0,1$ & $0,023 \pm 0,001$ & $<5,02$ & $1,42 \pm 0,05$ & $0,16 \pm 0,01$ & $<0,32$ & $19,4 \pm 0,2$ & $0,294 \pm 0,001$ \\
\hline $\mathrm{CCSH}$ & $0,91 \pm 0,04$ & $0,024 \pm 0,001$ & $<5,02$ & $1,06 \pm 0,04$ & $0,22 \pm 0,01$ & $0,71 \pm 0,03$ & $19,8 \pm 0,3$ & $0,332 \pm 0,002$ \\
\hline CCSPS & $0,74 \pm 0,04$ & $0,014 \pm 0,001$ & $6,7 \pm 0,4$ & $1,32 \pm 0,04$ & $0,16 \pm 0,01$ & $0,42 \pm 0,03$ & $20,8 \pm 0,4$ & $0,288 \pm 0,001$ \\
\hline
\end{tabular}




\begin{tabular}{ccccccccc}
\hline & $\mathbf{S b}$ & $\mathbf{S e}$ & $\mathbf{S m}$ & $\mathbf{S r}$ & $\mathbf{T h}$ & $\mathbf{U}$ & $\mathbf{V}$ \\
\hline CCDA & $0,025 \pm 0,001$ & $0,044 \pm 0,001$ & $<0,87$ & $117,2 \pm 0,4$ & $0,087 \pm 0,001$ & $0,19 \pm 0,01$ & $0,85 \pm 0,02$ & $20,7 \pm 0,2$ \\
CCDH & $0,022 \pm 0,001$ & $0,043 \pm 0,001$ & $<0,87$ & $95,7 \pm 0,4$ & $0,047 \pm 0,003$ & $0,12 \pm 0,01$ & $1,67 \pm 0,04$ & $19,0 \pm 0,2$ \\
CCDV & $0,023 \pm 0,001$ & $0,042 \pm 0,001$ & $<0,87$ & $121 \pm 1$ & $0,058 \pm 0,001$ & $0,19 \pm 0,01$ & $2,04 \pm 0,04$ & $18,7 \pm 0,2$ \\
CCEPA & $0,023 \pm 0,001$ & $0,044 \pm 0,001$ & $1,2 \pm 0,2$ & $111,2 \pm 0,4$ & $0,064 \pm 0,004$ & $0,19 \pm 0,01$ & $1,2 \pm 0,1$ & $19,4 \pm 0,3$ \\
CCHOV & $0,025 \pm 0,001$ & $0,045 \pm 0,001$ & $1,08 \pm 0,03$ & $110 \pm 1$ & $0,077 \pm 0,001$ & $0,18 \pm 0,01$ & $1,4 \pm 0,2$ & $20,2 \pm 0,3$ \\
CCM & $0,020 \pm 0,001$ & $0,042 \pm 0,001$ & $2,3 \pm 0,1$ & $131 \pm 1$ & $0,031 \pm 0,001$ & $<0,1$ & $1,02 \pm 0,04$ & $16,9 \pm 0,2$ \\
CCMFP & $0,019 \pm 0,001$ & $0,041 \pm 0,001$ & $1,2 \pm 0,2$ & $83,3 \pm 0,4$ & $0,024 \pm 0,001$ & $0,16 \pm 0,01$ & $1,52 \pm 0,04$ & $17,3 \pm 0,1$ \\
CCMS & $0,024 \pm 0,001$ & $0,045 \pm 0,001$ & $<0,87$ & $80 \pm 1$ & $0,079 \pm 0,001$ & $0,1 \pm 0,01$ & $0,94 \pm 0,04$ & $21,1 \pm 0,2$ \\
CCPLA & $0,021 \pm 0,001$ & $0,0422 \pm 0,0004$ & $<0,87$ & $85 \pm 1$ & $0,047 \pm 0,001$ & $0,13 \pm 0,01$ & $1,34 \pm 0,05$ & $18,8 \pm 0,3$ \\
CCPZ & $0,023 \pm 0,001$ & $0,043 \pm 0,001$ & $<0,87$ & $115 \pm 1$ & $0,055 \pm 0,001$ & $0,16 \pm 0,01$ & $1,3 \pm 0,2$ & $18,5 \pm 0,2$ \\
CCDLA & $0,023 \pm 0,001$ & $0,044 \pm 0,001$ & $1,2 \pm 0,3$ & $90 \pm 1$ & $0,077 \pm 0,003$ & $0,19 \pm 0,01$ & $1,05 \pm 0,02$ & $22,8 \pm 0,2$ \\
CCDLV & $0,019 \pm 0,001$ & $0,041 \pm 0,001$ & $1,35 \pm 0,03$ & $108 \pm 1$ & $0,016 \pm 0,001$ & $<0,1$ & $1,18 \pm 0,04$ & $15,9 \pm 0,2$ \\
CCFA & $0,024 \pm 0,001$ & $0,045 \pm 0,001$ & $1,3 \pm 0,4$ & $95,7 \pm 0,2$ & $0,089 \pm 0,001$ & $0,12 \pm 0,01$ & $1,00 \pm 0,04$ & $22,3 \pm 0,2$ \\
CCLMA & $0,019 \pm 0,001$ & $0,041 \pm 0,001$ & $1,4 \pm 0,3$ & $91,9 \pm 0,4$ & $0,025 \pm 0,001$ & $<0,1$ & $1,10 \pm 0,03$ & $17,8 \pm 0,2$ \\
CCMG & $0,019 \pm 0,001$ & $0,041 \pm 0,001$ & $<0,87$ & $82,6 \pm 0,2$ & $0,028 \pm 0,001$ & $0,18 \pm 0,01$ & $1,30 \pm 0,02$ & $18,1 \pm 0,1$ \\
CCMV & $0,022 \pm 0,001$ & $0,043 \pm 0,001$ & $<0,87$ & $80,4 \pm 0,4$ & $0,068 \pm 0,001$ & $0,16 \pm 0,01$ & $1,76 \pm 0,02$ & $20,8 \pm 0,3$ \\
CCFV & $0,019 \pm 0,001$ & $0,040 \pm 0,001$ & $<0,87$ & $113 \pm 1$ & $0,026 \pm 0,001$ & $0,19 \pm 0,01$ & $1,34 \pm 0,04$ & $16,7 \pm 0,3$ \\
CCSH & $0,018 \pm 0,001$ & $0,039 \pm 0,001$ & $<0,87$ & $93 \pm 1$ & $<0,036$ & $<0,1$ & $2,1 \pm 0,3$ & $16,8 \pm 0,2$ \\
CCSPS & $0,026 \pm 0,001$ & $0,043 \pm 0,001$ & $2,30 \pm 0,03$ & $100 \pm 1$ & $0,097 \pm 0,033$ & $<0,1$ & $0,76 \pm 0,04$ & $16,0 \pm 0,2$
\end{tabular}


APENDICE B.2 - Concentração dos elementos em $\mathrm{mg} \mathrm{kg}^{-1}$ (* em \%) nos cigarros aromatizados determinados por Espectrometria de raios- $X$

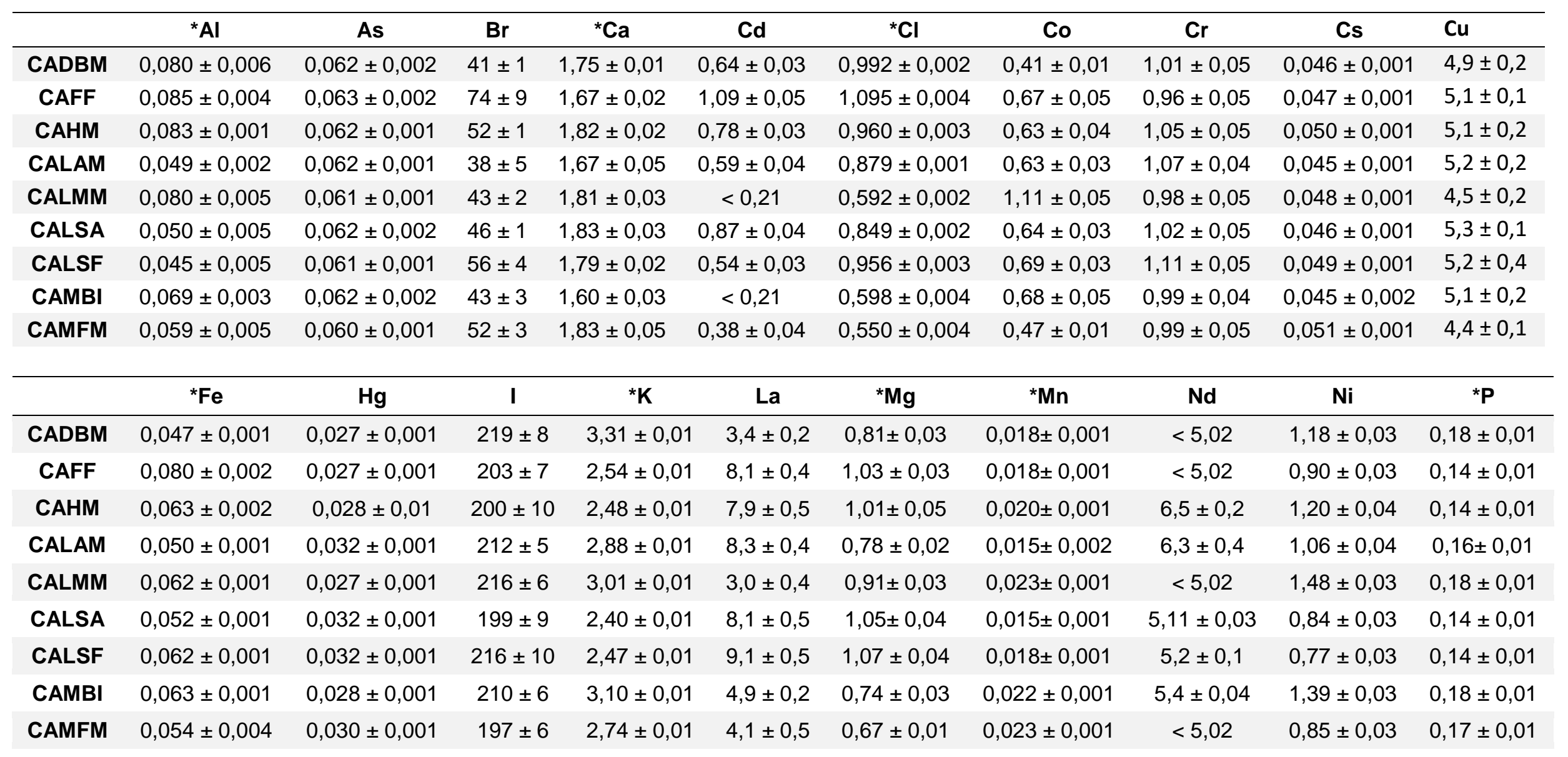




\begin{tabular}{|c|c|c|c|c|c|c|c|c|c|c|c|}
\hline & $\mathrm{Pb}$ & $\mathbf{R b}$ & ${ }^{*} S$ & $\mathrm{Sb}$ & $\mathrm{Se}$ & $\mathrm{Sm}$ & $\mathrm{Sr}$ & Th & $\mathbf{U}$ & V & $\mathrm{Zn}$ \\
\hline & ppm & ppm & $\%$ & ppm & ppm & ppm & $\mathrm{ppm}$ & ppm & ppm & ppm & ppm \\
\hline CADBM & $\begin{array}{c}1,12 \pm \\
0,05\end{array}$ & $\begin{array}{c}19,8 \pm \\
0,2\end{array}$ & $\begin{array}{c}0,336 \pm \\
0,001\end{array}$ & $\begin{array}{c}0,022 \pm \\
0,001\end{array}$ & $\begin{array}{c}0,043 \pm \\
0,001\end{array}$ & $<0,87$ & $128 \pm 1$ & $\begin{array}{c}0,060 \pm \\
0,001\end{array}$ & $\begin{array}{c}0,15 \pm \\
0,03\end{array}$ & $\begin{array}{c}0,95 \pm \\
0,04\end{array}$ & $\begin{array}{c}18,3 \pm \\
0,3\end{array}$ \\
\hline CAFF & $<0,32$ & $\begin{array}{c}20,1 \pm \\
0,3\end{array}$ & $\begin{array}{c}0,259 \pm \\
0,002\end{array}$ & $\begin{array}{c}0,024 \pm \\
0,001\end{array}$ & $\begin{array}{c}0,044 \pm \\
0,001\end{array}$ & $<0,87$ & $121 \pm 1$ & $\begin{array}{c}0,068 \pm \\
0,001\end{array}$ & $\begin{array}{c}0,18 \pm \\
0,04\end{array}$ & $\begin{array}{c}1,00 \pm \\
0,05\end{array}$ & $\begin{array}{c}19,1 \pm \\
0,2\end{array}$ \\
\hline CAHM & $\begin{array}{c}1,73 \pm \\
0,04\end{array}$ & $\begin{array}{c}19,5 \pm \\
0,2\end{array}$ & $\begin{array}{c}0,268 \pm \\
0,001\end{array}$ & $\begin{array}{c}0,024 \pm \\
0,001\end{array}$ & $\begin{array}{c}0,044 \pm \\
0,001\end{array}$ & $\begin{array}{c}1,01 \pm \\
0,05\end{array}$ & $113 \pm 1$ & $\begin{array}{c}0,078 \pm \\
0,001\end{array}$ & $\begin{array}{c}0,19 \pm \\
0,02\end{array}$ & $\begin{array}{c}1,68 \pm \\
0,05\end{array}$ & $\begin{array}{c}20,2 \pm \\
0,3\end{array}$ \\
\hline CALAM & $\begin{array}{c}0,44 \pm \\
0,05\end{array}$ & $\begin{array}{c}19,5 \pm \\
0,1\end{array}$ & $\begin{array}{c}0,311 \pm \\
0,001\end{array}$ & $\begin{array}{c}0,023 \pm \\
0,001\end{array}$ & $\begin{array}{c}0,044 \pm \\
0,001\end{array}$ & $\begin{array}{c}0,93 \pm \\
0,05\end{array}$ & $118 \pm 1$ & $\begin{array}{c}0,073 \pm \\
0,001\end{array}$ & $\begin{array}{c}0,15 \pm \\
0,05\end{array}$ & $\begin{array}{c}1,01 \pm \\
0,04\end{array}$ & $\begin{array}{c}19,9 \pm \\
0,2\end{array}$ \\
\hline CALMM & $\begin{array}{c}1,27 \pm \\
0,05\end{array}$ & $\begin{array}{c}19,9 \pm \\
0,2\end{array}$ & $\begin{array}{c}0,301 \pm \\
0,001\end{array}$ & $\begin{array}{c}0,021 \pm \\
0,001\end{array}$ & $\begin{array}{c}0,042 \pm \\
0,001\end{array}$ & $<0,87$ & $\begin{array}{c}92,1 \pm \\
0,4\end{array}$ & $\begin{array}{c}0,050 \pm \\
0,001\end{array}$ & $\begin{array}{c}0,25 \pm \\
0,03\end{array}$ & $\begin{array}{c}1,69 \pm \\
0,06\end{array}$ & $\begin{array}{c}18,1 \pm \\
0,1\end{array}$ \\
\hline CALSA & $\begin{array}{c}0,53 \pm \\
0,05\end{array}$ & $\begin{array}{c}19,6 \pm \\
0,2\end{array}$ & $\begin{array}{c}0,253 \pm \\
0,003\end{array}$ & $\begin{array}{c}0,023 \pm \\
0,001\end{array}$ & $\begin{array}{c}0,044 \pm \\
0,001\end{array}$ & $\begin{array}{c}1,33 \pm \\
0,03\end{array}$ & $105 \pm 1$ & $\begin{array}{c}0,073 \pm \\
0,002\end{array}$ & $\begin{array}{c}0,18 \pm \\
0,03\end{array}$ & $\begin{array}{c}1,25 \pm \\
0,04\end{array}$ & $\begin{array}{c}19,0 \pm \\
0,1\end{array}$ \\
\hline CALSF & $\begin{array}{c}1,55 \pm \\
0,05\end{array}$ & $\begin{array}{c}19,4 \pm \\
0,2\end{array}$ & $\begin{array}{c}0,276 \pm \\
0,002\end{array}$ & $\begin{array}{c}0,023 \pm \\
0,001\end{array}$ & $\begin{array}{c}0,044 \pm \\
0,001\end{array}$ & $1,8 \pm 0,1$ & $111 \pm 1$ & $\begin{array}{c}0,064 \pm \\
0,001\end{array}$ & $<0,1$ & $\begin{array}{c}0,69 \pm \\
0,04\end{array}$ & $9,4 \pm 0,2$ \\
\hline CAMBI & $\begin{array}{c}1,11 \pm \\
0,05\end{array}$ & $\begin{array}{c}19,8 \pm \\
0,4\end{array}$ & $\begin{array}{c}0,305 \pm \\
0,002\end{array}$ & $\begin{array}{c}0,021 \pm \\
0,001\end{array}$ & $\begin{array}{c}0,042 \pm \\
0,001\end{array}$ & $<0,87$ & $89 \pm 1$ & $\begin{array}{c}0,053 \pm \\
0,001\end{array}$ & $<0,1$ & $\begin{array}{c}1,70 \pm \\
0,04\end{array}$ & $\begin{array}{c}19,8 \pm \\
0,2\end{array}$ \\
\hline CAMFM & $\begin{array}{c}0,76 \pm \\
0,05\end{array}$ & $\begin{array}{c}19,8 \pm \\
0,2\end{array}$ & $\begin{array}{c}0,278 \pm \\
0,001\end{array}$ & $\begin{array}{c}0,022 \pm \\
0,001\end{array}$ & $\begin{array}{c}0,042 \pm \\
0,001\end{array}$ & $<0,87$ & $88 \pm 1$ & $\begin{array}{c}0,054 \pm \\
0,001\end{array}$ & $\begin{array}{c}0,23 \pm \\
0,05\end{array}$ & $\begin{array}{c}1,18 \pm \\
0,04\end{array}$ & $\begin{array}{c}18,4 \pm \\
0,2\end{array}$ \\
\hline
\end{tabular}


APENDICE B.3 - Concentração dos elementos em $\mathrm{mg} \mathrm{kg}^{-1}$ (* em \%) nos cigarros de palha determinados por Espectrometria de raios- $X$

\begin{tabular}{|c|c|c|c|c|c|c|c|c|c|c|}
\hline & ${ }^{*} \mathrm{Al}$ & As & $\mathrm{Br}$ & ${ }^{\star} \mathrm{Ca}$ & Cd & ${ }^{*} \mathrm{Cl}$ & Co & $\mathrm{Cr}$ & Cs & $\mathrm{Cu}$ \\
\hline CPPM & $0,077 \pm 0,002$ & $0,062 \pm 0,002$ & $18,0 \pm 0,5$ & $2,81 \pm 0,01$ & $1,47 \pm 0,03$ & $0,178 \pm 0,001$ & $0,75 \pm 0,01$ & $1,76 \pm 0,05$ & $0,061 \pm 0,001$ & $5,1 \pm 0,1$ \\
\hline CPC & $0,082 \pm 0,003$ & $0,061 \pm 0,001$ & $41 \pm 3$ & $3,41 \pm 0,03$ & $<0,21$ & $0,228 \pm 0,004$ & $0,72 \pm 0,03$ & $1,83 \pm 0,05$ & $0,063 \pm 0,001$ & $4,8 \pm 0,2$ \\
\hline CPCR & $0,085 \pm 0,004$ & $0,061 \pm 0,001$ & $64 \pm 1$ & $2,9 \pm 0,1$ & $<0,21$ & $0,62 \pm 0,01$ & $0,75 \pm 0,01$ & $1,7 \pm 0,1$ & $0,061 \pm 0,001$ & $4,3 \pm 0,2$ \\
\hline CPD & $0,084 \pm 0,003$ & $0,063 \pm 0,002$ & $75 \pm 3$ & $1,83 \pm 0,04$ & $<0,21$ & $0,719 \pm 0,004$ & $0,48 \pm 0,01$ & $1,00 \pm 0,01$ & $0,048 \pm 0,001$ & $4,4 \pm 0,2$ \\
\hline CPFN & $0,055 \pm 0,002$ & $0,060 \pm 0,001$ & $37 \pm 2$ & $1,95 \pm 0,02$ & $2,7 \pm 0,3$ & $0,558 \pm 0,002$ & $0,49 \pm 0,05$ & $1,1 \pm 0,1$ & $0,052 \pm 0,001$ & $5,1 \pm 0,3$ \\
\hline CPP & $0,085 \pm 0,002$ & $0,062 \pm 0,002$ & $53,5 \pm 0,2$ & $2,3 \pm 0,1$ & $0,85 \pm 0,04$ & $0,907 \pm 0,003$ & $1,1 \pm 0,2$ & $1,2 \pm 0,1$ & $0,057 \pm 0,001$ & $6,0 \pm 0,1$ \\
\hline CPPF & $0,048 \pm 0,003$ & $0,061 \pm 0,001$ & $20 \pm 2$ & $2,73 \pm 0,05$ & $1,0 \pm 0,1$ & $0,235 \pm 0,003$ & $0,65 \pm 0,04$ & $1,5 \pm 0,1$ & $0,057 \pm 0,001$ & $5,2 \pm 0,2$ \\
\hline CPPG & $0,145 \pm 0,004$ & $0,062 \pm 0,002$ & $31 \pm 2$ & $3,00 \pm 0,03$ & $<0,21$ & $0,467 \pm 0,003$ & $1,1 \pm 0,1$ & $1,65 \pm 0,05$ & $0,062 \pm 0,001$ & $5,1 \pm 0,1$ \\
\hline CPRN & $0,087 \pm 0,003$ & $0,062 \pm 0,002$ & $64 \pm 9$ & $1,82 \pm 0,04$ & $1,2 \pm 0,2$ & $0,622 \pm 0,003$ & $0,53 \pm 0,05$ & $0,95 \pm 0,02$ & $0,047 \pm 0,001$ & $5,5 \pm 0,2$ \\
\hline CPVC & $0,25 \pm 0,01$ & $0,060 \pm 0,001$ & $22 \pm 1$ & $2,87 \pm 0,01$ & $0,40 \pm 0,03$ & $0,117 \pm 0,005$ & $1,28 \pm 0,21$ & $1,7 \pm 0,1$ & $0,065 \pm 0,001$ & $4,8 \pm 0,3$ \\
\hline CРCPC & $0,082 \pm 0,003$ & $0,060 \pm 0,003$ & $29 \pm 1$ & $1,32 \pm 0,02$ & $0,48 \pm 0,05$ & $0,71 \pm 0,01$ & $0,58 \pm 0,03$ & $0,87 \pm 0,01$ & $0,042 \pm 0,002$ & $4,1 \pm 0,2$ \\
\hline CPCPE & $0,064 \pm 0,003$ & $0,061 \pm 0,001$ & $56 \pm 1$ & $1,99 \pm 0,01$ & $1,7 \pm 0,1$ & $0,68 \pm 0,01$ & $0,54 \pm 0,05$ & $0,93 \pm 0,04$ & $0,050 \pm 0,003$ & $5,0 \pm 0,2$ \\
\hline CPSP & $0,058 \pm 0,004$ & $0,062 \pm 0,001$ & $31 \pm 2$ & $2,83 \pm 0,03$ & $<0,21$ & $0,285 \pm 0,002$ & $1,2 \pm 0,2$ & $1,68 \pm 0,05$ & $0,053 \pm 0,001$ & $5,0 \pm 0,2$ \\
\hline CPTV & $0,065 \pm 0,005$ & $0,070 \pm 0,002$ & $35 \pm 39$ & $2,3 \pm 0,2$ & $2,0 \pm 0,3$ & $1,30 \pm 0,01$ & $0,67 \pm 0,05$ & $0,80 \pm 0,03$ & $0,043 \pm 0,001$ & $5,6 \pm 0,2$ \\
\hline
\end{tabular}




\begin{tabular}{|c|c|c|c|c|c|c|c|c|c|c|}
\hline & $\mathrm{Ee}$ & $g$ & I & ${ }^{*} K$ & La & ${ }^{*} \mathrm{Mg}$ & ${ }^{*} \mathrm{Mn}$ & Jd & $\mathrm{Ni}$ & ${ }^{*} P$ \\
\hline . & 1 & $30 \pm 0,001$ & 5 & 1 & & T & \pm & 2 & 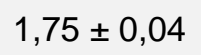 & 1 \\
\hline CPC & - & $6 \pm$ & & & & & & & & \\
\hline CPCR & $17 \pm$ & $5 \pm 0$ & & - & & & & & 04 & 001 \\
\hline CPD & $0,056 \pm 0$ & $7 \pm 0$ & 5 & $3 \pm$ & 2. & & & $<5,02$ & 01 & \\
\hline CPFI & $066 \pm 0,001$ & $35 \pm 0,001$ & $239 \pm 4$ & $102+0$ & $11 \pm 2$ & $3 \pm 0,004$ & $8 \pm 0,001$ & $3,4 \pm 0,4$ & $0 \pm 0,01$ & $0,097 \pm 0,004$ \\
\hline CPP & $0,158 \pm 0,001$ & $, 029 \pm 0,001$ & $211 \pm 13$ & $1,44 \pm 0,01$ & $3 \pm$ & $7 \pm 0,01$ & $0,019 \pm 0,001$ & $5,4 \pm 0,1$ & $1,41 \pm 0,01$ & $0,100 \pm$ \\
\hline CPPF & $0,070 \pm 0,001$ & $0,032 \pm 0,001$ & $199 \pm 7$ & $1,28 \pm 0,01$ & $18 \pm 1$ & $37 \pm 0,03$ & $0,011 \pm 0,001$ & $8,4 \pm 0,4$ & $2,4 \pm 0,4$ & $0,082 \pm$ \\
\hline CPPG & $0,156 \pm 0,005$ & $019 \pm 0,001$ & $214 \pm 5$ & $, 71 \pm 0,01$ & \pm 1 & 04 & $0,015 \pm 0,001$ & $<5,02$ & $1,1 \pm 0,3$ & 0,12 \\
\hline CPRN & $0,065 \pm 0,001$ & $0,029 \pm 0,001$ & $211 \pm 4$ & $3,13 \pm 0,01$ & $<1,4$ & $2 \pm 0,1$ & $0,027 \pm 0,001$ & $5,6 \pm 0,3$ & $1,2 \pm 0,2$ & $0,19 \pm 0,01$ \\
\hline CPVC & $0,202 \pm 0,004$ & $0,009 \pm 0,004$ & $212 \pm 8$ & $1,19 \pm 0,01$ & $39 \pm 2$ & $0,56 \pm 0,02$ & $0,105 \pm 0,005$ & $6,1 \pm 0,4$ & $1,4 \pm 0,3$ & $0,09 \pm 0,01$ \\
\hline СРCPC & $0,047 \pm 0,001$ & $0,027 \pm 0,001$ & $218 \pm 6$ & 3,26 & & 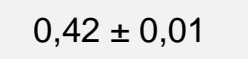 & 1 & $<5,02$ & 2 & 1 \\
\hline CPCPE & $0,066 \pm 0,001$ & $0,033 \pm 0,001$ & $214 \pm 5$ & $1,99 \pm 0,01$ & $<1,4$ & $0,59 \pm 0,04$ & $0,028 \pm 0,001$ & $5,9 \pm 0,2$ & $1,00 \pm 0,05$ & $0,12 \pm 0,01$ \\
\hline CPSP & $0,086 \pm 0,005$ & $0,030 \pm 0,001$ & $215 \pm 13$ & $1,25 \pm 0,02$ & $23 \pm 1$ & $0,66 \pm 0,04$ & $0,078 \pm 0,002$ & $6,1 \pm 0,4$ & $2,72 \pm 0,21$ & $0,087 \pm 0,004$ \\
\hline CPTV & $0,027 \pm 0,001$ & $0,032 \pm 0,001$ & $155 \pm 21$ & $3,66 \pm 0,01$ & $<1,4$ & $1,33 \pm 0,05$ & $0,08 \pm 0,01$ & $<5,02$ & $1,2 \pm 0,1$ & $0,22 \pm 0,01$ \\
\hline
\end{tabular}




\begin{tabular}{|c|c|c|c|c|c|c|c|c|c|c|c|}
\hline & $\mathbf{P b}$ & $\mathbf{R b}$ & ${ }^{*} S$ & Sb & $\mathrm{Se}$ & Sm & $\mathrm{Sr}$ & Th & $\mathbf{U}$ & V & $\mathrm{Zn}$ \\
\hline CPPM & $1,5 \pm 0,5$ & $\begin{array}{c}16,3 \pm \\
0,3\end{array}$ & $\begin{array}{c}0,252 \pm \\
0,003\end{array}$ & $\begin{array}{c}0,024 \pm \\
0,001\end{array}$ & $\begin{array}{c}0,045 \pm \\
0,001\end{array}$ & $<0,87$ & $\begin{array}{c}65,2 \pm \\
0,3\end{array}$ & $\begin{array}{c}0,095 \pm \\
0,002\end{array}$ & $0,14 \pm 0,01$ & $\begin{array}{c}1,65 \pm \\
0,04\end{array}$ & $24,4 \pm 0,1$ \\
\hline CPC & $<0,32$ & $\begin{array}{c}15,3 \pm \\
0,2\end{array}$ & $\begin{array}{c}0,358 \pm \\
0,001\end{array}$ & $\begin{array}{c}0,021 \pm \\
0,001\end{array}$ & $\begin{array}{c}0,042 \pm \\
0,001\end{array}$ & $<0,87$ & $\begin{array}{c}72,8 \pm \\
0,4\end{array}$ & $\begin{array}{c}0,047 \pm \\
0,002\end{array}$ & $0,17 \pm 0,02$ & $\begin{array}{c}1,88 \pm \\
0,04\end{array}$ & $19,9 \pm 0,2$ \\
\hline CPCR & $\begin{array}{c}0,63 \pm \\
0,03\end{array}$ & $\begin{array}{c}16,4 \pm \\
0,3\end{array}$ & $\begin{array}{c}0,330 \pm \\
0,002\end{array}$ & $\begin{array}{c}0,020 \pm \\
0,001\end{array}$ & $\begin{array}{c}0,041 \pm \\
0,001\end{array}$ & $<0,87$ & $\begin{array}{c}99,1 \pm \\
0,3\end{array}$ & $<0,036$ & $0,22 \pm 0,02$ & 2,0 0 0,1 & $17,6 \pm 0,2$ \\
\hline CPD & $<0,32$ & $\begin{array}{c}19,8 \pm \\
0,3\end{array}$ & $\begin{array}{c}0,266 \pm \\
0,001\end{array}$ & $\begin{array}{c}0,024 \pm \\
0,001\end{array}$ & $\begin{array}{c}0,043 \pm \\
0,001\end{array}$ & $\begin{array}{c}1,5 \pm \\
0,2\end{array}$ & $102 \pm 1$ & $\begin{array}{c}0,060 \pm \\
0,001\end{array}$ & $0,13 \pm 0,03$ & $\begin{array}{c}1,69 \pm \\
0,01\end{array}$ & $17,2 \pm 0,2$ \\
\hline CPFN & $\begin{array}{c}0,42 \pm \\
0,01\end{array}$ & $\begin{array}{c}19,4 \pm \\
0,4\end{array}$ & $\begin{array}{c}0,281 \pm \\
0,001\end{array}$ & $\begin{array}{c}0,029 \pm \\
0,001\end{array}$ & $\begin{array}{c}0,047 \pm \\
0,001\end{array}$ & $\begin{array}{c}1,3 \pm \\
0,2\end{array}$ & $\begin{array}{c}92,9 \pm \\
0,4\end{array}$ & $\begin{array}{c}0,122 \pm \\
0,001\end{array}$ & $0,18 \pm 0,02$ & $1,1 \pm 0,2$ & $22,8 \pm 0,3$ \\
\hline CPP & $1,2 \pm 0,2$ & $\begin{array}{c}19,1 \pm \\
0,2\end{array}$ & $\begin{array}{c}0,326 \pm \\
0,003\end{array}$ & $\begin{array}{c}0,025 \pm \\
0,001\end{array}$ & $\begin{array}{c}0,045 \pm \\
0,001\end{array}$ & $<0,87$ & $116 \pm 1$ & $\begin{array}{c}0,076 \pm \\
0,001\end{array}$ & $0,22 \pm 0,030$ & $2,2 \pm 0,1$ & $20,6 \pm 0,2$ \\
\hline CPPF & $1,4 \pm 0,3$ & $\begin{array}{c}17,0 \pm \\
0,2\end{array}$ & $\begin{array}{c}0,279 \pm \\
0,002\end{array}$ & $\begin{array}{c}0,023 \pm \\
0,001\end{array}$ & $\begin{array}{c}0,044 \pm \\
0,001\end{array}$ & $<0,87$ & $\begin{array}{c}70,0 \pm \\
0,4\end{array}$ & $\begin{array}{c}0,082 \pm \\
0,001\end{array}$ & $0,21 \pm 0,02$ & $\begin{array}{c}0,83 \pm \\
0,03\end{array}$ & $\begin{array}{c}22,7 \pm \\
0,02\end{array}$ \\
\hline CPPG & $\begin{array}{c}0,96 \pm \\
0,05\end{array}$ & $\begin{array}{c}16,4 \pm \\
0,2\end{array}$ & $\begin{array}{c}0,338 \pm \\
0,002\end{array}$ & $\begin{array}{c}0,019 \pm \\
0,001\end{array}$ & $\begin{array}{c}0,041 \pm \\
0,001\end{array}$ & $\begin{array}{c}1,8 \pm \\
0,2\end{array}$ & $\begin{array}{c}91,0 \pm \\
0,2\end{array}$ & $<0,036$ & $0,16 \pm 0,002$ & $\begin{array}{c}3,72 \pm \\
0,05\end{array}$ & $18,7 \pm 0,3$ \\
\hline CPRN & $1,7 \pm 0,3$ & $21 \pm 1$ & $\begin{array}{c}0,386 \pm \\
0,002\end{array}$ & $\begin{array}{c}0,027 \pm \\
0,001\end{array}$ & $\begin{array}{c}0,045 \pm \\
0,001\end{array}$ & $<0,87$ & $92 \pm 1$ & $\begin{array}{c}0,082 \pm \\
0,001\end{array}$ & $0,32 \pm 0,03$ & $1,1 \pm 0,2$ & $22,5 \pm 0,3$ \\
\hline CPVC & $1,8 \pm 0,03$ & $\begin{array}{c}16,4 \pm \\
0,2\end{array}$ & $\begin{array}{c}0,269 \pm \\
0,002\end{array}$ & $\begin{array}{c}0,021 \pm \\
0,001\end{array}$ & $\begin{array}{c}0,043 \pm \\
0,001\end{array}$ & $<0,87$ & $\begin{array}{c}64,1 \pm \\
0,3\end{array}$ & $\begin{array}{c}0,056 \pm \\
0,002\end{array}$ & $0,15 \pm 0,01$ & $\begin{array}{c}3,44 \pm \\
0,05\end{array}$ & $21,0 \pm 0,1$ \\
\hline СРСРC & $\begin{array}{l}0,40 \pm \\
0,004\end{array}$ & $\begin{array}{c}20,5 \pm \\
0,3\end{array}$ & $\begin{array}{c}0,291 \pm \\
0,002\end{array}$ & $\begin{array}{c}0,022 \pm \\
0,001\end{array}$ & $\begin{array}{c}0,043 \pm \\
0,001\end{array}$ & $\begin{array}{c}3,3 \pm \\
0,4\end{array}$ & $\begin{array}{c}91,0 \pm \\
0,5\end{array}$ & $\begin{array}{c}0,061 \pm \\
0,001\end{array}$ & $0,20 \pm 0,03$ & $\begin{array}{c}0,70 \pm \\
0,03\end{array}$ & $18,7 \pm 0,1$ \\
\hline CPCPE & $<0,32$ & $20 \pm 1$ & $0,33 \pm 0,01$ & $\begin{array}{c}0,026 \pm \\
0,001\end{array}$ & $\begin{array}{c}0,046 \pm \\
0,001\end{array}$ & $<0,87$ & $111 \pm 1$ & $\begin{array}{c}0,090 \pm \\
0,001\end{array}$ & $0,28 \pm 0,02$ & $1,0 \pm 0,1$ & $22,3 \pm 0,3$ \\
\hline CPSP & $<0,32$ & $\begin{array}{c}16,3 \pm \\
0,1\end{array}$ & $\begin{array}{c}0,297 \pm \\
0,003\end{array}$ & $\begin{array}{c}0,021 \pm \\
0,001\end{array}$ & $\begin{array}{c}0,043 \pm \\
0,001\end{array}$ & $\begin{array}{c}3,0 \pm \\
0,4\end{array}$ & $\begin{array}{c}74,0 \pm \\
0,2\end{array}$ & $\begin{array}{c}0,055 \pm \\
0,003\end{array}$ & $<0,1$ & $1,5 \pm 0,2$ & $20,8 \pm 0,3$ \\
\hline CPTV & $<0,32$ & $\begin{array}{c}20,6 \pm \\
0,5\end{array}$ & $0,45 \pm 0,01$ & $\begin{array}{c}0,043 \pm \\
0,004\end{array}$ & $\begin{array}{c}0,047 \pm \\
0,001\end{array}$ & $<0,87$ & $181 \pm 1$ & $\begin{array}{c}0,072 \pm \\
0,029\end{array}$ & $\begin{array}{l}0,33 \pm \\
0,0201\end{array}$ & $\begin{array}{c}0,97 \pm \\
0,05\end{array}$ & $24,8 \pm 0,3$ \\
\hline
\end{tabular}


APENDICE B.4 - Concentração dos elementos em mg kg-1 (* em \%) nos charutos determinados por Espectrometria de raios-x

\begin{tabular}{|c|c|c|c|c|c|c|c|c|c|c|c|}
\hline & ${ }^{*} \mathrm{Al}$ & As & $\mathrm{Br}$ & ${ }^{*} \mathrm{Ca}$ & Cd & ${ }^{*} \mathrm{Cl}$ & Co & $\mathrm{Cr}$ & Cs & $\mathrm{Cu}$ & ${ }^{\star} \mathrm{Fe}$ \\
\hline CHDF & $\begin{array}{c}0,101 \pm \\
0,002\end{array}$ & $\begin{array}{c}0,063 \pm \\
0,002\end{array}$ & $\begin{array}{c}94 \pm \\
3\end{array}$ & $\begin{array}{c}1,75 \pm \\
0,04\end{array}$ & $\begin{array}{c}0,38 \pm \\
0,02\end{array}$ & $1,47 \pm 0,01$ & $\begin{array}{c}0,38 \pm \\
0,05\end{array}$ & $\begin{array}{c}0,93 \pm \\
0,04\end{array}$ & $\begin{array}{c}0,048 \pm \\
0,001\end{array}$ & $5,3 \pm 0,1$ & $\begin{array}{c}0,045 \pm \\
0,003\end{array}$ \\
\hline CHDNC & $\begin{array}{c}0,085 \pm \\
0,004\end{array}$ & $\begin{array}{c}0,064 \pm \\
0,002\end{array}$ & $\begin{array}{c}82 \pm \\
4\end{array}$ & $\begin{array}{c}1,72 \pm \\
0,03\end{array}$ & $1,30 \pm 0,5$ & $\begin{array}{c}0,834 \pm \\
0,004\end{array}$ & $\begin{array}{c}0,41 \pm \\
0,05\end{array}$ & $\begin{array}{c}0,99 \pm \\
0,04\end{array}$ & $\begin{array}{c}0,046 \pm \\
0,001\end{array}$ & $5,4 \pm 0,2$ & $\begin{array}{c}0,053 \pm \\
0,004\end{array}$ \\
\hline CHDNE & $\begin{array}{l}0,084 \pm \\
0,003\end{array}$ & $\begin{array}{c}0,061 \pm \\
0,002\end{array}$ & $\begin{array}{c}38 \pm \\
2\end{array}$ & $\begin{array}{c}1,59 \pm \\
0,01\end{array}$ & $\begin{array}{c}0,61 \pm \\
0,01\end{array}$ & $\begin{array}{c}0,697 \pm \\
0,005\end{array}$ & $\begin{array}{c}0,84 \pm \\
0,05\end{array}$ & $\begin{array}{c}0,89 \pm \\
0,04\end{array}$ & $\begin{array}{c}0,047 \pm \\
0,001\end{array}$ & $\begin{array}{c}4,91 \pm \\
0,04\end{array}$ & $\begin{array}{c}0,038 \pm \\
0,001\end{array}$ \\
\hline CHMPC & $\begin{array}{c}0,082 \pm \\
0,003\end{array}$ & $\begin{array}{c}0,064 \pm \\
0,002\end{array}$ & $\begin{array}{c}92 \pm \\
9\end{array}$ & $\begin{array}{c}2,20 \pm \\
0,03\end{array}$ & $<0,21$ & $1,22 \pm 0,01$ & $\begin{array}{c}0,47 \pm \\
0,05\end{array}$ & $\begin{array}{c}1,26 \pm \\
0,04\end{array}$ & $\begin{array}{c}0,050 \pm \\
0,001\end{array}$ & $\begin{array}{c}5,78 \pm \\
0,10\end{array}$ & $\begin{array}{c}0,053 \pm \\
0,001\end{array}$ \\
\hline CHNI & $\mathrm{Hg}$ & I & ${ }^{*} \mathrm{~K}$ & & La & ${ }^{*} \mathrm{Mg}$ & ${ }^{*} \mathbf{M n}$ & $\mathbf{N d}$ & $\mathbf{N i}$ & ${ }^{*} \mathbf{P}$ & $\mathrm{Pb}$ \\
\hline CHDF & $0,026 \pm 0,001$ & $200 \pm 10$ & $3,66 \pm 0,0$ & & $8,7 \pm 0,5$ & $1,86 \pm 0,03$ & $0,012 \pm 0,001$ & $<5,02$ & $0,92 \pm 0,05$ & $0,18 \pm 0,01$ & $1,21 \pm 0,05$ \\
\hline CHDNC & $0,028 \pm 0,001$ & $211 \pm 11$ & $3,64 \pm 0,0$ & & $12,8 \pm 0,4$ & $2,08 \pm 0,05$ & $0,012 \pm 0,001$ & $5,8 \pm 0,5$ & $1,09 \pm 0,03$ & $0,25 \pm 0,01$ & $1,04 \pm 0,03$ \\
\hline CHMPE & $0,032 \pm 0,001$ & $204 \pm 9$ & $2,63 \pm 0,0$ & & $7,1 \pm 0,5$ & $2,71 \pm 0,03$ & $0,014 \pm 0,001$ & $<5,02$ & $1,09 \pm 0,03$ & $0,17 \pm 0,01$ & $0,87 \pm 0,05$ \\
\hline CHNI & $0,024 \pm 0,001$ & $211 \pm 14$ & $4,41 \pm 0,0$ & & $6,3 \pm 0,2$ & $1,44 \pm 0,03$ & $0,016 \pm 0,001$ & $<5,02$ & $1,10 \pm 0,03$ & $0,18 \pm 0,01$ & $0,44 \pm 0,05$ \\
\hline
\end{tabular}




\begin{tabular}{ccccccccccc}
\hline & $\mathbf{R b}$ & ${ }^{*}$ & Sb & Se & Sm & Sr & Th & U & V & Zn \\
\hline CHDF & $20,4 \pm 0,3$ & $0,459 \pm 0,004$ & $0,023 \pm 0,001$ & $0,044 \pm 0,001$ & $1,52 \pm 0,02$ & $167 \pm 1$ & $0,054 \pm 0,001$ & $0,21 \pm 0,03$ & $1,03 \pm 0,04$ & $21,4 \pm 0,3$ \\
CHDNC & $20,1 \pm 0,2$ & $0,488 \pm 0,002$ & $0,026 \pm 0,001$ & $0,045 \pm 0,001$ & $<0,87$ & $126 \pm 1$ & $0,076 \pm 0,001$ & $0,18 \pm 0,02$ & $1,07 \pm 0,04$ & $21,1 \pm 0,2$ \\
CHDNE & $20,3 \pm 0,3$ & $0,684 \pm 0,002$ & $0,022 \pm 0,001$ & $0,043 \pm 0,001$ & $1,68 \pm 0,01$ & $113 \pm 1$ & $0,053 \pm 0,001$ & $0,27 \pm 0,05$ & $1,11 \pm 0,04$ & $22,0 \pm 0,2$ \\
CHMPC & $18,4 \pm 0,1$ & $0,517 \pm 0,004$ & $0,022 \pm 0,001$ & $0,043 \pm 0,001$ & $2,11 \pm 0,05$ & $144 \pm 1$ & $0,045 \pm 0,001$ & $0,13 \pm 0,02$ & $0,72 \pm 0,04$ & $21,9 \pm 0,3$ \\
CHMPE & $18,2 \pm 0,2$ & $0,495 \pm 0,002$ & $0,025 \pm 0,001$ & $0,045 \pm 0,001$ & $<0,87$ & $164 \pm 1$ & $0,069 \pm 0,001$ & $0,21 \pm 0,05$ & $1,09 \pm 0,01$ & $24,6 \pm 0,3$ \\
CHNI & $20,4 \pm 0,4$ & $0,592 \pm 0,004$ & $0,024 \pm 0,001$ & $0,044 \pm 0,001$ & $<0,87$ & $150 \pm 1$ & $0,058 \pm 0,001$ & $0,23 \pm 0,01$ & $1,05 \pm 0,04$ & $21,6 \pm 0,2$
\end{tabular}


APENDICE B.5 - Concentração dos elementos em $\mathrm{mg} \mathrm{kg}^{-1}$ (* em \%) nos fumos de corda e rapés determinados por Espectrometria de raios- $X$

\begin{tabular}{|c|c|c|c|c|c|c|c|c|c|c|}
\hline & ${ }^{\star} \mathrm{Al}$ & As & $\mathrm{Br}$ & ${ }^{\star} \mathrm{Ca}$ & Cd & ${ }^{\star} \mathrm{Cl}$ & Co & $\mathrm{Cr}$ & Cs & $\mathrm{Cu}$ \\
\hline FCA2 & $\begin{array}{c}0,126 \pm \\
0,003\end{array}$ & $0,063 \pm 0,003$ & $61 \pm 4$ & $\begin{array}{c}1,75 \pm \\
0,01\end{array}$ & $2,8 \pm 0,3$ & $\begin{array}{c}0,777 \pm \\
0,004\end{array}$ & $0,41 \pm 0,04$ & $0,87 \pm 0,07$ & $\begin{array}{c}0,0472 \pm \\
0,0004\end{array}$ & $6,5 \pm 0,3$ \\
\hline FCA6 & $\begin{array}{c}0,115 \pm \\
0,004\end{array}$ & $0,061 \pm 0,001$ & $28 \pm 1$ & $\begin{array}{c}1,47 \pm \\
0,01\end{array}$ & $1,6 \pm 0,2$ & $\begin{array}{c}0,910 \pm \\
0,002\end{array}$ & $0,52 \pm 0,04$ & $0,82 \pm 0,01$ & $\begin{array}{c}0,0458 \pm \\
0,0004\end{array}$ & $6,5 \pm 0,3$ \\
\hline FCA7 & $\begin{array}{c}0,116 \pm \\
0,004\end{array}$ & $0,065 \pm 0,002$ & $51 \pm 2$ & $\begin{array}{c}1,66 \pm \\
0,02\end{array}$ & $2,8 \pm 0,1$ & $\begin{array}{c}0,632 \pm \\
0,004\end{array}$ & $0,60 \pm 0,05$ & $0,89 \pm 0,03$ & $0,047 \pm 0,002$ & $7,8 \pm 0,1$ \\
\hline FCAAL & $\begin{array}{c}0,089 \pm \\
0,003\end{array}$ & $0,062 \pm 0,001$ & $51 \pm 2$ & $\begin{array}{c}1,73 \pm \\
0,03\end{array}$ & $3,4 \pm 0,5$ & $0,75 \pm 0,01$ & $0,43 \pm 0,04$ & $0,89 \pm 0,03$ & $0,046 \pm 0,002$ & $5,4 \pm 0,1$ \\
\hline FCACE & $\begin{array}{c}0,016 \pm \\
0,003\end{array}$ & $0,062 \pm 0,002$ & $39 \pm 2$ & $\begin{array}{c}1,45 \pm \\
0,03\end{array}$ & $2,5 \pm 0,4$ & $\begin{array}{c}0,244 \pm \\
0,002\end{array}$ & $0,6 \pm 0,1$ & $0,97 \pm 0,05$ & $0,046 \pm 0,001$ & $4,8 \pm 0,2$ \\
\hline FCAMO & $\begin{array}{c}0,029 \pm \\
0,005\end{array}$ & $\begin{array}{c}0,0608 \pm \\
0,0004\end{array}$ & $43 \pm 1$ & $\begin{array}{c}1,45 \pm \\
0,03\end{array}$ & $2,0 \pm 0,1$ & $\begin{array}{c}0,324 \pm \\
0,002\end{array}$ & $0,36 \pm 0,02$ & $0,87 \pm 0,04$ & $\begin{array}{c}0,0438 \pm \\
0,0004\end{array}$ & $4,5 \pm 0,2$ \\
\hline FCA5 & $\begin{array}{c}0,143 \pm \\
0,003\end{array}$ & $0,065 \pm 0,001$ & $239 \pm 29$ & $1,7 \pm 0,1$ & $<0,21$ & $1,74 \pm 0,01$ & $0,49 \pm 0,05$ & $0,89 \pm 0,04$ & $0,045 \pm 0,001$ & $5,8 \pm 0,2$ \\
\hline FCA10 & $\begin{array}{c}0,110 \pm \\
0,003\end{array}$ & $0,063 \pm 0,002$ & $34 \pm 1$ & $1,7 \pm 0,1$ & $4,0 \pm 0,2$ & $\begin{array}{c}0,867 \pm \\
0,004\end{array}$ & $0,7 \pm 0,1$ & $0,87 \pm 0,04$ & $0,049 \pm 0,003$ & $7,3 \pm 0,4$ \\
\hline FCDBH & $\begin{array}{c}0,039 \pm \\
0,005\end{array}$ & $0,063 \pm 0,002$ & $49 \pm 1$ & $\begin{array}{c}1,65 \pm \\
0,02\end{array}$ & $2,3 \pm 0,4$ & $\begin{array}{c}0,418 \pm \\
0,001\end{array}$ & $\begin{array}{c}0,505 \pm \\
0,005\end{array}$ & $1,0 \pm 0,1$ & $0,046 \pm 0,001$ & $5,2 \pm 0,2$ \\
\hline FCDD & $\begin{array}{c}0,075 \pm \\
0,005\end{array}$ & $0,063 \pm 0,001$ & $103 \pm 5$ & $\begin{array}{c}1,74 \pm \\
0,04\end{array}$ & $1,8 \pm 0,3$ & $\begin{array}{c}0,889 \pm \\
0,004\end{array}$ & $0,52 \pm 0,05$ & $0,95 \pm 0,03$ & $0,047 \pm 0,001$ & $5,4 \pm 0,2$ \\
\hline FCDHT & $\begin{array}{c}0,070 \pm \\
0,002\end{array}$ & $0,061 \pm 0,001$ & $40 \pm 1$ & $\begin{array}{c}1,91 \pm \\
0,03\end{array}$ & $1,8 \pm 0,2$ & $\begin{array}{c}0,494 \pm \\
0,003\end{array}$ & $0,61 \pm 0,01$ & $1,1 \pm 0,1$ & $0,049 \pm 0,001$ & $4,4 \pm 0,2$ \\
\hline FCDJ & $\begin{array}{c}0,079 \pm \\
0,002\end{array}$ & $0,066 \pm 0,001$ & $166 \pm 5$ & $\begin{array}{c}1,94 \pm \\
0,05\end{array}$ & $1,8 \pm 0,1$ & $\begin{array}{c}1,171 \pm \\
0,004\end{array}$ & $0,36 \pm 0,04$ & $0,93 \pm 0,01$ & $0,047 \pm 0,001$ & $6,0 \pm 0,2$ \\
\hline FCDMP & $\begin{array}{c}0,088 \pm \\
0,003\end{array}$ & $0,061 \pm 0,001$ & $53 \pm 2$ & $\begin{array}{c}2,41 \pm \\
0,05\end{array}$ & $2,0 \pm 0,2$ & $0,81 \pm 0,004$ & $0,85 \pm 0,04$ & $1,0 \pm 0,1$ & $0,054 \pm 0,001$ & $5,6 \pm 0,2$ \\
\hline FCDPG & $\begin{array}{c}0,075 \pm \\
0,003\end{array}$ & $0,063 \pm 0,001$ & $59 \pm 2$ & $\begin{array}{c}1,60 \pm \\
0,05\end{array}$ & $2,8 \pm 0,2$ & $\begin{array}{c}0,710 \pm \\
0,003\end{array}$ & $1,1 \pm 0,2$ & $1,0 \pm 0,1$ & $0,047 \pm 0,001$ & $5,3 \pm 0,4$ \\
\hline FCDTT & $\begin{array}{c}0,011 \pm \\
0,001\end{array}$ & $0,060 \pm 0,001$ & $21 \pm 3$ & $\begin{array}{c}1,65 \pm \\
0,02\end{array}$ & $2,4 \pm 0,4$ & $\begin{array}{c}0,400 \pm \\
0,003\end{array}$ & $0,57 \pm 0,03$ & $1,08 \pm 0,05$ & $0,046 \pm 0,001$ & $4,8 \pm 0,1$ \\
\hline FCG & $\begin{array}{c}0,081 \pm \\
0,003\end{array}$ & $0,060 \pm 0,001$ & $31 \pm 4$ & $\begin{array}{c}2,30 \pm \\
0,03\end{array}$ & $<0,21$ & $\begin{array}{c}0,521 \pm \\
0,003\end{array}$ & $0,54 \pm 0,05$ & $1,10 \pm 0,05$ & $0,055 \pm 0,001$ & $5,7 \pm 0,1$ \\
\hline
\end{tabular}




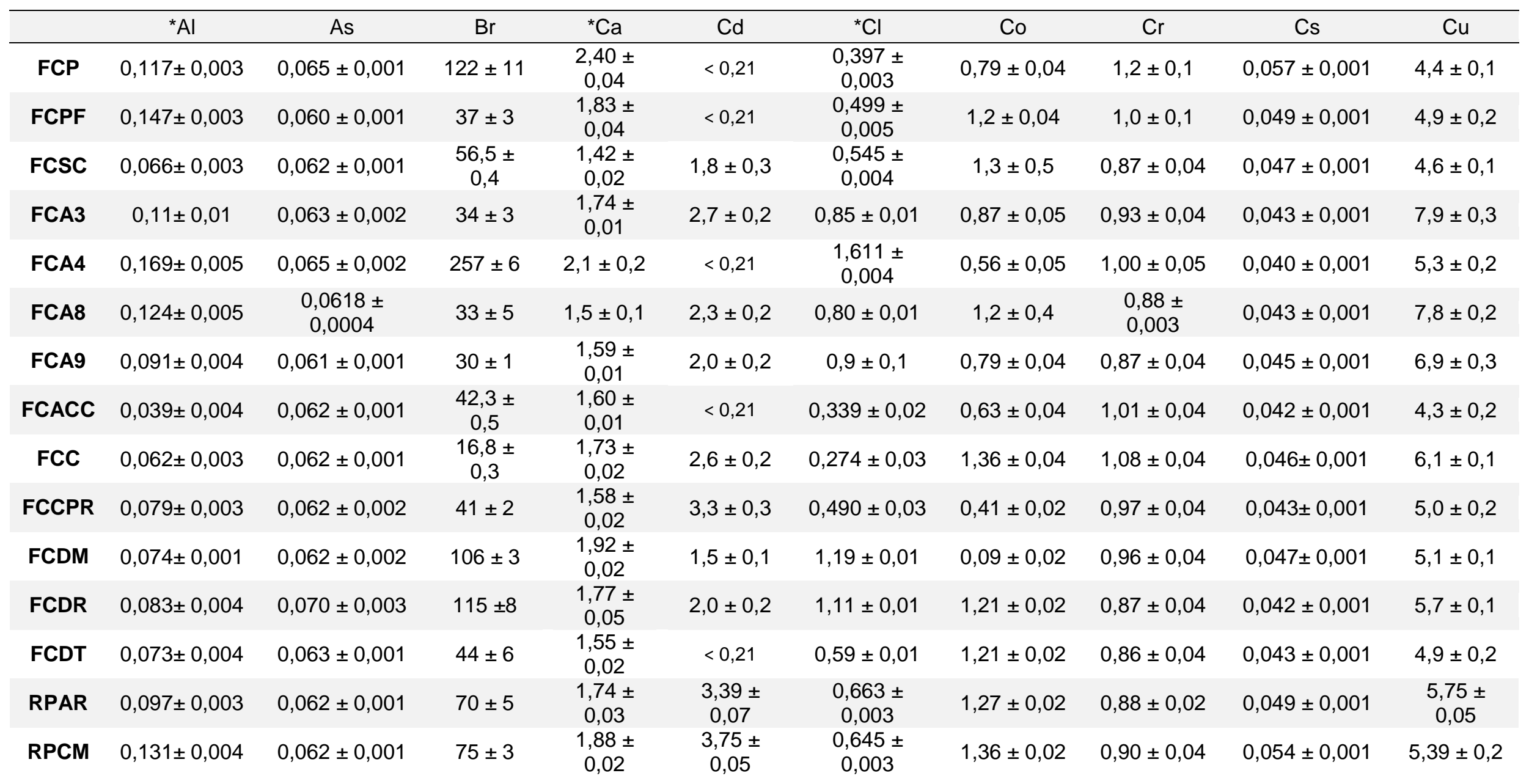




\begin{tabular}{|c|c|c|c|c|c|c|c|c|c|c|c|}
\hline & ${ }^{*} \mathrm{Fe}$ & $\mathrm{Hg}$ & I & ${ }^{*} \mathrm{~K}$ & La & *Mg & *Mn & Nd & $\mathrm{Ni}$ & ${ }^{\star} \mathbf{P}$ & $\mathrm{Pb}$ \\
\hline FCA2 & $0,063 \pm 0,002$ & $0,027 \pm 0,001$ & $214 \pm 10$ & $3,74 \pm 0,01$ & $4,9 \pm 0,6$ & $1,57 \pm 0,04$ & $0,022 \pm 0,001$ & $<5,02$ & $<0,60$ & $0,15 \pm 0,01$ & $<0,32$ \\
\hline FCA6 & $0,083 \pm 0,002$ & $0,026 \pm 0,001$ & $212 \pm 5$ & $3,72 \pm 0,01$ & $13,3 \pm 0,4$ & $1,68 \pm 0,04$ & $0,013 \pm 0,001$ & $<5,02$ & $<0,60$ & $0,112 \pm 0,004$ & $1,1 \pm 0,1$ \\
\hline FCA7 & $0,062 \pm 0,001$ & $0,027 \pm 0,001$ & $225 \pm 1$ & $3,42 \pm 0,01$ & $7,9 \pm 0,4$ & $1,9 \pm 0,1$ & $0,020 \pm 0,001$ & $7,0 \pm 0,5$ & $0,9 \pm 0,3$ & $0,108 \pm 0,004$ & $1,5 \pm 0,2$ \\
\hline FCAAL & $0,044 \pm 0,001$ & $0,032 \pm 0,001$ & $194 \pm 7$ & $3,87 \pm 0,02$ & $9,5 \pm 0,2$ & $1,0 \pm 0,1$ & $0,010 \pm 0,001$ & $<5,02$ & $1,12 \pm 0,02$ & $0,27 \pm 0,01$ & $1,6 \pm 0,4$ \\
\hline FCACE & $0,032 \pm 0,003$ & $0,039 \pm 0,001$ & $199 \pm 8$ & $1,41 \pm 0,01$ & $11 \pm 1$ & $0,13 \pm 0,02$ & $0,019 \pm 0,002$ & $13,3 \pm 0,4$ & $1,9 \pm 0,3$ & $0,11 \pm 0,01$ & $<0,32$ \\
\hline FCAMO & $0,039 \pm 0,002$ & $0,036 \pm 0,001$ & $208 \pm 9$ & $1,78 \pm 0,01$ & $4,4 \pm 0,4$ & $0,12 \pm 0,04$ & $0,022 \pm 0,001$ & $8,7 \pm 0,3$ & $1,4 \pm 0,6$ & $0,138 \pm 0,004$ & $<0,32$ \\
\hline FCA5 & $0,0510 \pm 0,0004$ & $1,3 \pm 0,2$ & $164 \pm 3$ & $4,58 \pm 0,01$ & $<1,4$ & $1,7 \pm 0,1$ & $0,068 \pm 0,003$ & $<5,02$ & $0,8 \pm 0,1$ & $0,17 \pm 0,01$ & $<0,32$ \\
\hline FCA10 & $0,053 \pm 0,003$ & $0,030 \pm 0,001$ & $230 \pm 8$ & $3,39 \pm 0,02$ & $17 \pm 1$ & $1,7 \pm 0,1$ & $0,011 \pm 0,002$ & $9,3 \pm 0,3$ & $1,5 \pm 0,3$ & $0,12 \pm 0,01$ & $1,0 \pm 0,3$ \\
\hline FCDBH & $0,0643 \pm 0,0003$ & $0,035 \pm 0,001$ & $220 \pm 5$ & $1,98 \pm 0,01$ & $9,9 \pm 0,5$ & $0,53 \pm 0,02$ & $0,017 \pm 0,001$ & $8,5 \pm 0,3$ & $1,7 \pm 0,2$ & $0,12 \pm 0,01$ & $1,2 \pm 0,1$ \\
\hline FCDD & $0,048 \pm 0,002$ & $0,030 \pm 0,001$ & $222 \pm 12$ & $2,98 \pm 0,01$ & $<1,4$ & $1,15 \pm 0,05$ & $0,031 \pm 0,002$ & $6,2 \pm 0,2$ & $0,8 \pm 0,3$ & $0,17 \pm 0,01$ & $0,8 \pm 0,1$ \\
\hline FCDHT & $0,058 \pm 0,001$ & $0,032 \pm 0,001$ & $220 \pm 6$ & $2,07 \pm 0,01$ & $2,1 \pm 0,3$ & $0,75 \pm 0,03$ & $0,026 \pm 0,001$ & $6,2 \pm 0,4$ & $1,2 \pm 0,2$ & $0,15 \pm 0,01$ & $<0,32$ \\
\hline FCDJ & $0,046 \pm 0,001$ & $0,030 \pm 0,001$ & $191 \pm 12$ & $3,56 \pm 0,01$ & $<1,4$ & $1,54 \pm 0,03$ & $0,040 \pm 0,003$ & $<5,02$ & $1,3 \pm 0,2$ & $0,22 \pm 0,01$ & $<0,32$ \\
\hline FCDMP & $0,107 \pm 0,001$ & $0,029 \pm 0,001$ & $223 \pm 12$ & $2,59 \pm 0,01$ & $<1,4$ & $0,91 \pm 0,03$ & $0,022 \pm 0,001$ & $<5,02$ & $<0,60$ & $0,15 \pm 0,01$ & $<0,32$ \\
\hline FCDPG & $0,039 \pm 0,004$ & $0,034 \pm 0,001$ & $219 \pm 10$ & $2,35 \pm 0,01$ & $<1,4$ & $0,93 \pm 0,03$ & $0,018 \pm 0,001$ & $11 \pm 1$ & $1,2 \pm 0,2$ & $0,12 \pm 0,01$ & $<0,32$ \\
\hline FCDTT & $0,028 \pm 0,002$ & $0,040 \pm 0,001$ & $215 \pm 10$ & $1,41 \pm 0,01$ & $17 \pm 1$ & $0,35 \pm 0,03$ & $0,010 \pm 0,001$ & $12,0 \pm 0,4$ & $1,7 \pm 0,2$ & $0,12 \pm 0,01$ & $<0,32$ \\
\hline FCG & $0,064 \pm 0,003$ & $0,028 \pm 0,001$ & $220 \pm 4$ & $2,36 \pm 0,01$ & $17 \pm 1$ & $0,67 \pm 0,03$ & $0,016 \pm 0,001$ & $<5,02$ & $1,1 \pm 0,2$ & $0,16 \pm 0,01$ & $<0,32$ \\
\hline FCP & $0,118 \pm 0,001$ & $0,024 \pm 0,001$ & $200 \pm 5$ & $2,46 \pm 0,01$ & $13,8 \pm 0,5$ & $0,86 \pm 0,01$ & $0,018 \pm 0,001$ & $<5,02$ & $1,0 \pm 0,2$ & $0,15 \pm 0,01$ & $<0,32$ \\
\hline FCPF & $0,096 \pm 0,002$ & $0,023 \pm 0,001$ & $217 \pm 4$ & $3,70 \pm 0,01$ & $6,4 \pm 0,5$ & $0,12 \pm 0,02$ & $0,021 \pm 0,001$ & $5,5 \pm 0,2$ & $<0,60$ & $0,19 \pm 0,01$ & $<0,32$ \\
\hline
\end{tabular}




\begin{tabular}{cccccccccccc}
\hline & ${ }^{*} \mathrm{Fe}$ & $\mathrm{Hg}$ & $\mathrm{I}$ & ${ }^{* K}$ & $\mathrm{La}$ & ${ }^{*} \mathrm{Mg}$ & ${ }^{*} \mathrm{Mn}$ & $\mathrm{Nd}$ & $\mathrm{Ni}$ & ${ }^{*} \mathrm{P}$ & $\mathrm{Pb}$ \\
\hline FCSC & $0,081 \pm 0,002$ & $0,033 \pm 0,001$ & $215 \pm 6$ & $2,00 \pm 0,01$ & $15 \pm 1$ & $0,21 \pm 0,04$ & $0,017 \pm 0,001$ & $7,3 \pm 0,3$ & $0,61 \pm 0,05$ & $0,13 \pm 0,01$ & $<0,32$ \\
FCA3 & $0,047 \pm 0,005$ & $0,028 \pm 0,001$ & $231 \pm 8$ & $4,2 \pm 0,1$ & $12 \pm 1$ & $2,2 \pm 0,1$ & $0,076 \pm 0,004$ & $<5,02$ & $1,3 \pm 0,1$ & $0,14 \pm 0,01$ & $<0,32$ \\
FCA4 & $0,078 \pm 0,003$ & $0,019 \pm 0,001$ & $172 \pm 5$ & $3,63 \pm 0,01$ & $<1,4$ & $1,7 \pm 0,1$ & $0,081 \pm 0,004$ & $<5,02$ & $<0,60$ & $0,17 \pm 0,01$ & $<0,32$ \\
FCA8 & $0,061 \pm 0,003$ & $0,025 \pm 0,001$ & $234 \pm 17$ & $3,94 \pm 0,01$ & $15 \pm 1$ & $1,99 \pm 0,01$ & $0,081 \pm 0,004$ & $<5,02$ & $<0,60$ & $0,13 \pm 0,01$ & $1,0 \pm 0,01$ \\
FCA9 & $0,058 \pm 0,003$ & $0,030 \pm 0,001$ & $226 \pm 5$ & $3,56 \pm 0,01$ & $10 \pm 1$ & $1,46 \pm 0,01$ & $0,015 \pm 0,001$ & $5,9 \pm 0,3$ & $0,74 \pm 0,05$ & $0,11 \pm 0,01$ & $0,9 \pm 0,1$ \\
FCACC & $0,033 \pm 0,001$ & $0,034 \pm 0,001$ & $215 \pm 4$ & $2,11 \pm 0,01$ & $7,6 \pm 0,4$ & $0,39 \pm 0,01$ & $0,014 \pm 0,001$ & $6,0 \pm 0,3$ & $1,9 \pm 0,4$ & $0,17 \pm 0,01$ & $1,8 \pm 0,5$ \\
FCC & $0,062 \pm 0,001$ & $0,034 \pm 0,001$ & $214 \pm 9$ & $1,12 \pm 0,01$ & $26,8 \pm 0,5$ & $0,66 \pm 0,02$ & $0,007 \pm 0,001$ & $11,8 \pm 0,3$ & $1,6 \pm 0,3$ & $0,062 \pm 0,004$ & $1,1 \pm 0,1$ \\
FCCPR & $0,029 \pm 0,001$ & $0,033 \pm 0,001$ & $212 \pm 17$ & $3,34 \pm 0,02$ & $13 \pm 1$ & $1,29 \pm 0,05$ & $0,015 \pm 0,001$ & $8,1 \pm 0,4$ & $1,3 \pm 0,3$ & $0,45 \pm 0,04$ & $1,2 \pm 0,1$ \\
FCDM & $0,077 \pm 0,001$ & $0,031 \pm 0,001$ & $200 \pm 9$ & $3,06 \pm 0,02$ & $<1,4$ & $0,95 \pm 0,05$ & $0,030 \pm 0,001$ & $<5,02$ & $1,02 \pm 0,4$ & $0,162 \pm 0,004$ & $1,3 \pm 0,1$ \\
FCDR & $0,042 \pm 0,005$ & $0,030 \pm 0,001$ & $195 \pm 10$ & $2,99 \pm 0,03$ & $3,0 \pm 0,3$ & $1,03 \pm 0,04$ & $0,024 \pm 0,001$ & $<5,02$ & $<0,60$ & $0,152 \pm 0,004$ & $1,2 \pm 0,1$ \\
FCDT & $0,047 \pm 0,005$ & $0,028 \pm 0,001$ & $202 \pm 8$ & $2,61 \pm 0,02$ & $13 \pm 1$ & $0,68 \pm 0,04$ & $0,012 \pm 0,001$ & $5,8 \pm 0,4$ & $1,6 \pm 0,1$ & $0,150 \pm 0,004$ & $1,5 \pm 0,1$ \\
RPAR & $0,144 \pm 0,005$ & $0,031 \pm 0,001$ & $212 \pm 9$ & $2,72 \pm 0,01$ & $4,5 \pm 0,4$ & $1,26 \pm 0,04$ & $0,025 \pm 0,001$ & $9,31 \pm 0,05$ & $<0,60$ & $0,25 \pm 0,01$ & $0,83 \pm 0,02$ \\
RPCM & $0,147 \pm 0,004$ & $0,028 \pm 0,001$ & $219 \pm 12$ & $3,04 \pm 0,02$ & $7,1 \pm 0,4$ & $1,39 \pm 0,04$ & $0,025 \pm 0,001$ & $7,66 \pm 0,05$ & $2,21 \pm 0,03$ & $0,327 \pm 0,004$ & $1,45 \pm 0,04$
\end{tabular}




\begin{tabular}{|c|c|c|c|c|c|c|c|c|c|c|}
\hline & $\mathbf{R b}$ & *S & Sb & $\mathrm{Se}$ & $\mathrm{Sm}$ & $\mathrm{Sr}$ & Th & $\mathbf{U}$ & V & $\mathrm{Zn}$ \\
\hline FCA2 & $20,6 \pm 0,3$ & $0,341 \pm 0,002$ & $0,0228 \pm 0,0004$ & $0,047 \pm 0,001$ & $2,8 \pm 0,7$ & $127 \pm 1$ & $0,097 \pm 0,001$ & $0,25 \pm 0,01$ & $1,6 \pm 0,2$ & $24,3 \pm 0,2$ \\
\hline FCA6 & $20,8 \pm 0,3$ & $0,330 \pm 0,002$ & $0,026 \pm 0,001$ & $0,046 \pm 0,001$ & $<0,87$ & $131 \pm 1$ & $0,079 \pm 0,001$ & $0,25 \pm 0,01$ & $1,8 \pm 0,1$ & $23,0 \pm 0,3$ \\
\hline FCA7 & $20,28 \pm 0,04$ & $0,329 \pm 0,001$ & $0,029 \pm 0,001$ & $0,0472 \pm 0,004$ & $<0,87$ & $113 \pm 1$ & $0,110 \pm 0,001$ & $0,24 \pm 0,01$ & $1,48 \pm 0,06$ & $25,9 \pm 0,3$ \\
\hline FCAAL & $19,8 \pm 0,3$ & $0,512 \pm 0,003$ & $0,032 \pm 0,001$ & $0,048 \pm 0,001$ & $<0,87$ & $109 \pm 1$ & $0,116 \pm 0,001$ & $0,25 \pm 0,01$ & $0,55 \pm 0,04$ & $18,7 \pm 0,1$ \\
\hline FCACE & $20,2 \pm 0,2$ & $0,241 \pm 0,001$ & $0,028 \pm 0,001$ & $0,047 \pm 0,001$ & $<0,87$ & $64,5 \pm 0,4$ & $0,133 \pm 0,001$ & $0,20 \pm 0,01$ & $1,0 \pm 0,2$ & $22,4 \pm 0,3$ \\
\hline FCAMO & $20,4 \pm 0,2$ & $0,241 \pm 0,001$ & $0,027 \pm 0,001$ & $0,0458 \pm 0,0004$ & $<0,87$ & $69,3 \pm 0,4$ & $0,112 \pm 0,001$ & $<0,1$ & $1,0 \pm 0,1$ & $19,3 \pm 0,2$ \\
\hline FCA5 & $20,6 \pm 0,3$ & $0,392 \pm 0,001$ & $0,028 \pm 0,002$ & $0,043 \pm 0,001$ & $<0,87$ & $187,5 \pm 0,3$ & $0,037 \pm 0,001$ & $0,17 \pm 0,01$ & $0,93 \pm 0,03$ & $25 \pm 1$ \\
\hline FCA10 & $20,4 \pm 0,2$ & $0,352 \pm 0,003$ & $0,0318 \pm 0,0004$ & $0,049 \pm 0,001$ & $<0,87$ & $135 \pm 1$ & $0,129 \pm 0,002$ & $0,24 \pm 0,01$ & $1,0 \pm 0,1$ & $27,7 \pm 0,3$ \\
\hline FCDBH & $20,1 \pm 0,5$ & $0,269 \pm 0,001$ & $0,027 \pm 0,001$ & $0,046 \pm 0,001$ & $<0,87$ & $76,7 \pm 0,2$ & $0,107 \pm 0,001$ & $<0,1$ & $1,0 \pm 0,2$ & $21,3 \pm 0,2$ \\
\hline FCDD & $20,1 \pm 0,5$ & $0,423 \pm 0,002$ & $0,029 \pm 0,001$ & $0,046 \pm 0,002$ & $1,5 \pm 0,5$ & $122 \pm 1$ & $0,091 \pm 0,001$ & $0,20 \pm 0,01$ & $1,1 \pm 0,2$ & $25 \pm 1$ \\
\hline FCDHT & $19,4 \pm 0,3$ & $0,260 \pm 0,001$ & $0,026 \pm 0,001$ & $0,046 \pm 0,001$ & $<0,87$ & $86 \pm 1$ & $0,092 \pm 0,002$ & $0,18 \pm 0,01$ & $1,1 \pm 0,2$ & $18,8 \pm 0,2$ \\
\hline FCDJ & $20,2 \pm 0,5$ & $0,464 \pm 0,001$ & $0,031 \pm 0,001$ & $0,046 \pm 0,001$ & $<0,87$ & $144 \pm 1$ & $0,082 \pm 0,002$ & $0,19 \pm 0,01$ & $0,77 \pm 0,02$ & $24 \pm 1$ \\
\hline FCDMP & $19,6 \pm 0,4$ & $0,385 \pm 0,002$ & $0,028 \pm 0,001$ & $0,046 \pm 0,001$ & $<0,87$ & $132 \pm 1$ & $0,084 \pm 0,001$ & $0,24 \pm 0,01$ & $2,4 \pm 0,3$ & $23,1 \pm 0,4$ \\
\hline FCDPG & $20,4 \pm 0,4$ & $0,385 \pm 0,002$ & $0,030 \pm 0,001$ & $0,048 \pm 0,001$ & $<0,87$ & $108,7 \pm 0,4$ & $0,126 \pm 0,002$ & $0,15 \pm 0,01$ & $1,4 \pm 0,1$ & $24,9 \pm 0,5$ \\
\hline FCDTT & $19,4 \pm 0,2$ & $0,266 \pm 0,001$ & $0,028 \pm 0,001$ & $0,047 \pm 0,001$ & $<0,87$ & $70,3 \pm 0,4$ & $0,133 \pm 0,002$ & $<0,1$ & $0,5 \pm 0,1$ & $20,5 \pm 0,1$ \\
\hline FCG & $19,1 \pm 0,2$ & $0,255 \pm 0,002$ & $0,023 \pm 0,001$ & $0,043 \pm 0,001$ & $<0,87$ & $93,0 \pm 0,3$ & $0,071 \pm 0,001$ & $0,22 \pm 0,01$ & $1,2 \pm 0,3$ & $25,8 \pm 0,3$ \\
\hline FCP & $18,8 \pm 0,4$ & $0,370 \pm 0,002$ & $0,026 \pm 0,001$ & $0,044 \pm 0,001$ & $<0,87$ & $92,0 \pm 0,4$ & $0,063 \pm 0,001$ & $0,23 \pm 0,01$ & $2,1 \pm 0,3$ & $18,3 \pm 0,2$ \\
\hline FCPF & $19,7 \pm 0,4$ & $0,437 \pm 0,001$ & $0,027 \pm 0,001$ & $0,046 \pm 0,001$ & $<0,87$ & $91,0 \pm 0,5$ & $0,0878 \pm 0,0004$ & $<0,1$ & $1,3 \pm 0,3$ & $21,3 \pm 0,2$ \\
\hline FCSC & $20,4 \pm 0,3$ & $0,382 \pm 0,001$ & $0,027 \pm 0,004$ & $0,047 \pm 0,001$ & $<0,87$ & $80,3 \pm 0,2$ & $0,101 \pm 0,001$ & $0,35 \pm 0,01$ & $1,6 \pm 0,2$ & $23,7 \pm 0,2$ \\
\hline FCA3 & $20,2 \pm 0,2$ & $0,377 \pm 0,004$ & $0,028 \pm 0,001$ & $0,047 \pm 0,001$ & $2,8 \pm 0,1$ & $137 \pm 1$ & $0,088 \pm 0,002$ & $0,19 \pm 0,01$ & $0,88 \pm 0,05$ & $23,9 \pm 0,3$ \\
\hline
\end{tabular}




\begin{tabular}{|c|c|c|c|c|c|c|c|c|c|c|}
\hline & $\mathrm{Rb}$ & *S & $\mathrm{Sb}$ & $\mathrm{Se}$ & $\mathrm{Sm}$ & $\mathrm{Sr}$ & Th & $U$ & V & $\mathrm{Zn}$ \\
\hline FCA4 & $19,62 \pm 0,3$ & $0,346 \pm 0,003$ & $0,033 \pm 0,003$ & $0,045 \pm 0,001$ & $<0,87$ & $187 \pm 1$ & $0,063 \pm 0,002$ & $<0,1$ & $2,2 \pm 0,3$ & $26 \pm 2$ \\
\hline FCA8 & $20,6 \pm 0,4$ & $0,374 \pm 0,001$ & $0,027 \pm 0,003$ & $0,046 \pm 0,001$ & $<0,87$ & $129 \pm 1$ & $0,086 \pm 0,001$ & $0,18 \pm 0,01$ & $1,6 \pm 0,1$ & $23,8 \pm 0,3$ \\
\hline FCA9 & $20,6 \pm 0,2$ & $0,030 \pm 0,001$ & $0,027 \pm 0,001$ & $0,046 \pm 0,001$ & $1,6 \pm 0,5$ & $114,2 \pm 0,5$ & $0,103 \pm 0,001$ & $<0,1$ & $1,2 \pm 0,3$ & $25,4 \pm 0,4$ \\
\hline FCACC & $20,0 \pm 0,1$ & $0,317 \pm 0,001$ & $0,025 \pm 0,001$ & $0,045 \pm 0,001$ & $2,5 \pm 0,5$ & $71,6 \pm 0,3$ & $0,090 \pm 0,001$ & $<0,1$ & $0,86 \pm 0,01$ & $17,5 \pm 0,3$ \\
\hline FCC & $19,5 \pm 0,2$ & $0,336 \pm 0,002$ & $0,027 \pm 0,001$ & $0,047 \pm 0,001$ & $<0,87$ & $72,6 \pm 0,3$ & $0,126 \pm 0,001$ & $0,37 \pm 0,01$ & $1,1 \pm 0,3$ & $20,8 \pm 0,2$ \\
\hline FCCPR & $20,1 \pm 0,2$ & $0,462 \pm 0,002$ & $0,030 \pm 0,001$ & $0,048 \pm 0,001$ & $<0,87$ & $94,4 \pm 0,2$ & $0,119 \pm 0,001$ & $0,33 \pm 0,01$ & $0,40 \pm 0,05$ & $22,3 \pm 0,1$ \\
\hline FCDM & $20,2 \pm 0,4$ & $0,408 \pm 0,002$ & $0,027 \pm 0,001$ & $0,048 \pm 0,001$ & $1,0 \pm 0,2$ & $149 \pm 0,5$ & $0,079 \pm 0,001$ & $0,29 \pm 0,01$ & $1,40 \pm 0,3$ & $21,7 \pm 0,2$ \\
\hline FCDR & $20,6 \pm 0,5$ & $0,392 \pm 0,002$ & $0,029 \pm 0,002$ & $0,046 \pm 0,001$ & $5,4 \pm 0,5$ & $145 \pm 1$ & $0,088 \pm 0,001$ & $0,28 \pm 0,01$ & $1,27 \pm 0,03$ & $21,6 \pm 0,4$ \\
\hline FCDT & $20,4 \pm 0,5$ & $0,274 \pm 0,002$ & $0,023 \pm 0,001$ & $0,044 \pm 0,001$ & $<0,87$ & $89 \pm 1$ & $0,065 \pm 0,001$ & $0,18 \pm 0,01$ & $1,1 \pm 0,2$ & $19,7 \pm 0,2$ \\
\hline RPAR & $20,5 \pm 0,2$ & $0,46 \pm 0,01$ & $0,032 \pm 0,001$ & $0,049 \pm 0,001$ & $<0,87$ & $108 \pm 1$ & $0,12 \pm 0,01$ & $0,26 \pm 0,04$ & $1,9 \pm 0,2$ & $19,7 \pm 0,2$ \\
\hline RPCM & $20,4 \pm 0,3$ & $0,4493 \pm 0,002$ & $0,035 \pm 0,001$ & $0,049 \pm 0,001$ & $<0,87$ & $<0,65$ & $0,11 \pm 0,01$ & $0,340 \pm 0,004$ & $3,65 \pm 0,2$ & $23,5 \pm 0,5$ \\
\hline
\end{tabular}


APENDICE C.1 - Concentração dos radionuclídeos ${ }^{226} \mathrm{Ra},{ }^{210} \mathrm{~Pb},{ }^{210} \mathrm{Po},{ }^{238} \mathrm{U},{ }^{228} \mathrm{Ra}$ e ${ }^{232} \mathrm{Th}_{\mathrm{em}} \mathrm{mBq} \mathrm{g}^{-1}$ nos cigarros aromatizados.

\begin{tabular}{ccccccc}
\hline & ${ }^{226} \mathbf{R a}$ & ${ }^{210} \mathbf{P b}$ & ${ }^{210} \mathbf{P o}$ & ${ }^{238} \mathbf{U}$ & ${ }^{228} \mathbf{R a}$ & ${ }^{323} \mathbf{T h}$ \\
\hline CADBM & $5,9 \pm 0,3$ & $25 \pm 2$ & $6,3 \pm 0,5$ & $1,8 \pm 0,4$ & $39 \pm 2$ & $0,245 \pm 0,004$ \\
CAFF & $13 \pm 1$ & $19 \pm 11$ & $19 \pm 1$ & $2,2 \pm 0,5$ & $38 \pm 2$ & $0,276 \pm 0,004$ \\
CAHM & $8 \pm 1$ & $20 \pm 2$ & $19 \pm 1$ & $2,3 \pm 0,3$ & $39 \pm 6$ & $0,318 \pm 0,004$ \\
CALAM & $4,7 \pm 0,2$ & $<4,9 \pm 0,4$ & $7 \pm 1$ & $1,9 \pm 0,6$ & $34 \pm 2$ & $0,299 \pm 0,004$ \\
CALMM & $5,8 \pm 0,2$ & $24 \pm 14$ & $18 \pm 1$ & $3,1 \pm 0,4$ & $34 \pm 4$ & $0,202 \pm 0,004$ \\
CALSA & $11 \pm 1$ & $<4,9 \pm 0,4$ & $17 \pm 1$ & $2,2 \pm 0,4$ & $31 \pm 5$ & $0,30 \pm 0,01$ \\
CALSF & $14 \pm 1$ & $24 \pm 1$ & $15 \pm 1$ & - & $26 \pm 5$ & $0,261 \pm 0,004$ \\
CAMBI & $13 \pm 1$ & $19 \pm 2$ & $21 \pm 1$ & - & $46 \pm 2$ & $0,217 \pm 0,004$ \\
CAMFM & $6,2 \pm 0,2$ & $24 \pm 1$ & $22 \pm 1$ & $2,8 \pm 0,6$ & $69 \pm 10$ & $0,220 \pm 0,004$
\end{tabular}

- não determinado 
APENDICE C. 2 - Concentração dos radionuclídeos ${ }^{226} \mathrm{Ra},{ }^{210} \mathrm{~Pb},{ }^{210} \mathrm{Po},{ }^{238} \mathrm{U},{ }^{228} \mathrm{Ra}$ $\mathrm{e}^{232} \mathrm{Th}$ em mBq g-1 nos cigarros não aromatizados.

\begin{tabular}{ccccccc}
\hline & ${ }^{226} \mathbf{R a}$ & ${ }^{210} \mathbf{P b}$ & ${ }^{210} \mathbf{P o}$ & ${ }^{238} \mathbf{U}$ & ${ }^{228} \mathbf{R a}$ & ${ }^{323} \mathbf{T h}$ \\
\hline CCDA & $9 \pm 3$ & $19 \pm 6$ & $20 \pm 1$ & $2,4 \pm 0,1$ & $42 \pm 9$ & $0,354 \pm 0,004$ \\
CCDH & $8 \pm 1$ & $17 \pm 5$ & $17 \pm 1$ & $1,5 \pm 0,1$ & $39 \pm 3$ & $0,240 \pm 0,01$ \\
CCDV & $9 \pm 3$ & $20 \pm 1$ & $25 \pm 1$ & $2,4 \pm 0,1$ & $45 \pm 6$ & $0,238 \pm 0,004$ \\
CCEPA & $11 \pm 1$ & $25 \pm 1$ & $14 \pm 1$ & $2,4 \pm 0,1$ & $40 \pm 8$ & $0,26 \pm 0,02$ \\
CCFA & $7 \pm 1$ & $15 \pm 5$ & $18 \pm 1$ & $1,5 \pm 0,1$ & $41 \pm 3$ & $0,363 \pm 0,004$ \\
CCFV & $11 \pm 1$ & $20 \pm 2$ & $21 \pm 1$ & $2,4 \pm 0,1$ & $43 \pm 3$ & $0,108 \pm 0,004$ \\
CCHOV & $10 \pm 1$ & $15 \pm 3$ & $23 \pm 1$ & $2,3 \pm 0,1$ & $41 \pm 4$ & $0,313 \pm 0,004$ \\
CCLMA & $11 \pm 2$ & $26 \pm 2$ & $22 \pm 1$ & $1,3 \pm 0,1$ & $38 \pm 6$ & $0,100 \pm 0,001$ \\
CCM & $10 \pm 2$ & $23 \pm 6$ & $24 \pm 1$ & - & $44 \pm 6$ & $0,127 \pm 0,004$ \\
CCMFP & $13 \pm 2$ & $25 \pm 3$ & $20 \pm 1$ & $2,0 \pm 0,1$ & $40 \pm 6$ & $0,096 \pm 0,004$ \\
CCMG & $8 \pm 2$ & $22 \pm 1$ & $23 \pm 2$ & $2,3 \pm 0,1$ & $36 \pm 5$ & $0,113 \pm 0,004$ \\
CCMGA & $10 \pm 1$ & $21 \pm 2$ & $21 \pm 1$ & $2,4 \pm 0,1$ & $47 \pm 3$ & $0,323 \pm 0,004$ \\
CCMV & $9 \pm 1$ & $25 \pm 1$ & $19 \pm 1$ & $2,0 \pm 0,1$ & $34 \pm 2$ & $0,277 \pm 0,0041$ \\
CCPLA & $12 \pm 1$ & $24 \pm 2$ & $22 \pm 1$ & $1,6 \pm 0,1$ & $38 \pm 3$ & $0,193 \pm 0,004$ \\
CCPZ & $12 \pm 1$ & $25 \pm 1$ & $24 \pm 2$ & $2,0 \pm 0,1$ & $40 \pm 3$ & $0,222 \pm 0,004$ \\
CCSPS & $11 \pm 2$ & $15 \pm 2$ & $17 \pm 1$ & - & $36 \pm 6$ & $0,40 \pm 0,01$
\end{tabular}

- não determinado 
APENDICE C.3 - Concentração dos radionuclídeos ${ }^{226} \mathrm{Ra},{ }^{210} \mathrm{~Pb},{ }^{210} \mathrm{Po},{ }^{238} \mathrm{U},{ }^{228} \mathrm{Ra}$ $\mathrm{e}^{232} \mathrm{Th}$ em $\mathrm{mBq} \mathrm{g}^{-1}$ nos cigarros de palha.

\begin{tabular}{ccccccc}
\hline & ${ }^{226} \mathbf{R a}$ & ${ }^{210} \mathbf{P b}$ & ${ }^{210} \mathbf{P o}$ & ${ }^{238} \mathbf{U}$ & ${ }^{228} \mathbf{R a}$ & ${ }^{323} \mathbf{T h}$ \\
\hline CPC & $6 \pm 0$ & $17 \pm 2$ & $19 \pm 1$ & $2,2 \pm 0,1$ & $47 \pm 4$ & $0,19 \pm 0,01$ \\
CPCPC & $13 \pm 3$ & $37 \pm 3$ & $35 \pm 2$ & $2,5 \pm 0,1$ & $37 \pm 3$ & $0,247 \pm 0,004$ \\
CPCPE & $12 \pm 1$ & $32 \pm 2$ & $18 \pm 1$ & $3,6 \pm 0,1$ & $52 \pm 2$ & $0,367 \pm 0,004$ \\
CPD & $13 \pm 2$ & $19 \pm 9$ & $19 \pm 1$ & $1,6 \pm 0,1$ & $65 \pm 6$ & $0,243 \pm 0,004$ \\
CPFN & $19 \pm 1$ & $38 \pm 4$ & $22 \pm 1$ & $2,3 \pm 0,1$ & $71 \pm 4$ & $0,498 \pm 0,004$ \\
CPP & $13 \pm 2$ & $17 \pm 1$ & $21 \pm 1$ & $2,7 \pm 0,1$ & $50 \pm 13$ & $0,311 \pm 0,004$ \\
CPPE & $6 \pm 2$ & $41 \pm 5$ & $23 \pm 1$ & $2,8 \pm 0,1$ & $59 \pm 3$ & $0,139 \pm 0,004$ \\
CPPG & $6 \pm 1$ & $31 \pm 3$ & $11 \pm 1$ & $2,0 \pm 0,1$ & $60 \pm 3$ & $0,128 \pm 0,004$ \\
CPPM & $9,3+0,5$ & $40 \pm 3$ & $20 \pm 1$ & $1,8 \pm 0,1$ & $47+2$ & $0,386 \pm 0,01$ \\
CPRN & $12 \pm 1$ & $29 \pm 3$ & $26 \pm 2$ & $4,0 \pm 0,1$ & $52 \pm 3$ & $0,334 \pm 0,004$ \\
CPSP & $8 \pm 0$ & $24 \pm 5$ & $13 \pm 1$ & $2,7 \pm 0,1$ & $54 \pm 4$ & $0,23 \pm 0,01$ \\
CPTV & $22 \pm 7$ & $25 \pm 4$ & $14 \pm 1$ & $4,1 \pm 0,1$ & $58 \pm 1$ & $0,35 \pm 0,01$ \\
CPVC & $12 \pm 1$ & $55 \pm 5$ & $11 \pm 1$ & $1,9 \pm 0,1$ & $81 \pm 4$ & $0,23 \pm 0,01$
\end{tabular}

APENDICE C.4 - Concentração dos radionuclídeos ${ }^{226} \mathrm{Ra},{ }^{210} \mathrm{~Pb},{ }^{210} \mathrm{Po},{ }^{238} \mathrm{U},{ }^{228} \mathrm{Ra}$

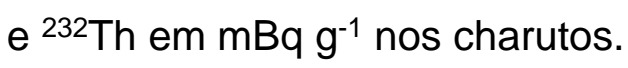

\begin{tabular}{ccccccc}
\hline & ${ }^{226} \mathbf{R a}$ & ${ }^{210} \mathbf{P b}$ & ${ }^{210} \mathbf{P o}$ & ${ }^{238} \mathbf{U}$ & ${ }^{228} \mathbf{R a}$ & ${ }^{323} \mathbf{T h}$ \\
\hline CHDF & $20 \pm 1$ & $6,2 \pm 0,5$ & $16 \pm 2$ & $2,6 \pm 0,4$ & $55 \pm 4$ & $0,221 \pm 0,004$ \\
CHDNE & $25 \pm 1$ & $10 \pm 1$ & $15 \pm 1$ & $2,3 \pm 0,3$ & $52 \pm 3$ & $0,310 \pm 0,004$ \\
CHDNC & $21 \pm 1$ & $12 \pm 1$ & $14 \pm 2$ & $3,4 \pm 0,6$ & $53 \pm 1$ & $0,215 \pm 0,004$ \\
CHMPC & $28 \pm 1$ & $22 \pm 2$ & $22 \pm 1$ & $1,6 \pm 0,3$ & $67 \pm 2$ & $0,183 \pm 0,004$ \\
CHMPE & $18 \pm 1$ & $13 \pm 1$ & $17 \pm 1$ & $2,6 \pm 0,6$ & $78 \pm 4$ & $0,279 \pm 0,004$ \\
CHNI & $19 \pm 1$ & $14 \pm 1$ & $13 \pm 1$ & $2,9 \pm 0,1$ & $29 \pm 1$ & $0,238 \pm 0,004$
\end{tabular}


APENDICE C.5 - Concentração dos radionuclídeos ${ }^{226} \mathrm{Ra},{ }^{210} \mathrm{~Pb},{ }^{210} \mathrm{Po},{ }^{238} \mathrm{U},{ }^{228} \mathrm{Ra}$ e ${ }^{232}$ Th em $\mathrm{mBq} \mathrm{g}^{-1}$ nos fumos de corda e rapés.

\begin{tabular}{|c|c|c|c|c|c|c|}
\hline Amostra & ${ }^{226} \mathrm{Ra}$ & ${ }^{210} \mathrm{~Pb}$ & ${ }^{210} \mathrm{Po}$ & ${ }^{238} \mathrm{U}$ & ${ }^{228} \mathbf{R a}$ & ${ }^{323} \mathrm{Th}$ \\
\hline FCA1 & $9 \pm 2$ & $49 \pm 1$ & $16 \pm 1$ & $3,1 \pm 0,1$ & $35 \pm 6$ & $0,321 \pm 0,004$ \\
\hline FCA2 & $10 \pm 1$ & $51 \pm 1$ & $18 \pm 1$ & $3,1 \pm 0,1$ & $44 \pm 8$ & $0,393 \pm 0,004$ \\
\hline FCA3 & $9 \pm 1$ & $50 \pm 6$ & $18 \pm 1$ & $2,4 \pm 0,1$ & $40 \pm 6$ & $0,359 \pm 0,01$ \\
\hline FCA4 & $9 \pm 1$ & $56 \pm 8$ & $24 \pm 1$ & - & $41 \pm 1$ & $0,257 \pm 0,01$ \\
\hline FCA5 & $11 \pm 1$ & $63 \pm 9$ & $22 \pm 2$ & $2,1 \pm 0,1$ & $47 \pm 3$ & $0,151 \pm 0,004$ \\
\hline FCA7 & $14 \pm 5$ & $55 \pm 9$ & $14 \pm 1$ & $3,0 \pm 0,2$ & $54 \pm 6$ & $0,447 \pm 0,004$ \\
\hline FCA8 & $12 \pm 5$ & $57 \pm 5$ & $15 \pm 1$ & $2,3 \pm 0,1$ & $42 \pm 1$ & $0,348 \pm 0,004$ \\
\hline FCA9 & $13 \pm 1$ & $78 \pm 2$ & $19 \pm 2$ & - & $37 \pm 2$ & $0,420 \pm 0,004$ \\
\hline FCA10 & $8 \pm 2$ & $61 \pm 6$ & $17 \pm 1$ & $3,0 \pm 0,2$ & $34 \pm 4$ & $0,527 \pm 0,002$ \\
\hline FCAAL & $40 \pm 6$ & $25 \pm 1$ & $21 \pm 1$ & $3,1 \pm 0,1$ & $152 \pm 1$ & $0,471 \pm 0,004$ \\
\hline FCACC & $7 \pm 2$ & $12 \pm 8$ & $14 \pm 2$ & - & $29 \pm 2$ & $0,365 \pm 0,004$ \\
\hline FCACE & $9 \pm 2$ & $17 \pm 4$ & $11 \pm 1$ & $2,5 \pm 0,1$ & $28 \pm 6$ & $0,542 \pm 0,004$ \\
\hline FCAMO & $8 \pm 1$ & $10 \pm 1$ & $12 \pm 1$ & - & $31 \pm 6$ & $0,456 \pm 0,004$ \\
\hline FCC & $16 \pm 2$ & $12 \pm 2$ & $16 \pm 2$ & $4,6 \pm 0,1$ & $41 \pm 10$ & $0,511 \pm 0,004$ \\
\hline FCCPR & $17 \pm 8$ & $13 \pm 2$ & $18 \pm 1$ & $4,1 \pm 0,1$ & $66 \pm 2$ & $0,486 \pm 0,004$ \\
\hline FCDBH & $7 \pm 0$ & $30 \pm 5$ & $24 \pm 1$ & $2,9 \pm 0,2$ & $32 \pm 1$ & $0,435 \pm 0,004$ \\
\hline FCDD & $7 \pm 2$ & $18 \pm 1$ & $10 \pm 1$ & $2,5 \pm 0,1$ & $44 \pm 2$ & $0,369 \pm 0,004$ \\
\hline FCDHT & $15 \pm 3$ & $29 \pm 2$ & $21 \pm 1$ & $2,3 \pm 0,1$ & $40 \pm 6$ & $0,374 \pm 0,01$ \\
\hline FCDJ & $11 \pm 2$ & $50 \pm 3$ & $15 \pm 2$ & $2,4 \pm 0,1$ & $42 \pm 11$ & $0,332 \pm 0,01$ \\
\hline FCDM & $13 \pm 1$ & $66 \pm 0$ & $17 \pm 1$ & $3,6 \pm 0,1$ & $42 \pm 1$ & $0,320 \pm 0,004$ \\
\hline FCDMP & $19 \pm 6$ & $5 \pm 1$ & $21 \pm 1$ & $3,0 \pm 0,3$ & $59 \pm 5$ & $0,334 \pm 0,004$ \\
\hline FCDPG & $12 \pm 2$ & $10 \pm 0$ & $11 \pm 1$ & $1,9 \pm 0,4$ & $54 \pm 5$ & $0,514 \pm 0,01$ \\
\hline FCDR & $13 \pm 3$ & $16 \pm 6$ & $11 \pm 1$ & - & $49 \pm 4$ & $0,357 \pm 0,004$ \\
\hline FCDT & $10 \pm 1$ & $21 \pm 2$ & $23 \pm 1$ & $2,7 \pm 0,1$ & $39 \pm 4$ & $0,265 \pm 0,004$ \\
\hline FCDTT & $6 \pm 2$ & $26 \pm 4$ & $26 \pm 1$ & - & $30 \pm 2$ & $0,543 \pm 0,01$ \\
\hline FCG & $9 \pm 2$ & $27 \pm 1$ & $23 \pm 1$ & $2,8 \pm 0,1$ & $50 \pm 9$ & $0,287 \pm 0,004$ \\
\hline $\mathrm{FCl}$ & $11 \pm 1$ & $14 \pm 1$ & $15 \pm 1$ & $3,5 \pm 0,1$ & $41 \pm 6$ & - \\
\hline FCP & $15 \pm 1$ & $21 \pm 1$ & $18 \pm 1$ & $2,9 \pm 0,1$ & $53 \pm 9$ & $0,257 \pm 0,004$ \\
\hline FCPF & $15 \pm 1$ & $37 \pm 1$ & $28 \pm 1$ & - & $59 \pm 6$ & $0,358 \pm 0,001$ \\
\hline FCSC & $14 \pm 1$ & $14 \pm 1$ & $10 \pm 1$ & $4,4 \pm 0,1$ & $78 \pm 4$ & $0,411 \pm 0,004$ \\
\hline RPAR & $20 \pm 1$ & $35 \pm 2$ & $18 \pm 1$ & $3,2 \pm 0,1$ & $23 \pm 2$ & $0,487 \pm 0,004$ \\
\hline RPCM & $16 \pm 1$ & $34 \pm 1$ & $17 \pm 2$ & $4,3 \pm 0,1$ & $12 \pm 1$ & $0,460 \pm 0,004$ \\
\hline
\end{tabular}

- não determinado 